\title{
VISUALIZATION AND ANALYSIS OF ELECTRODYNAMIC BEHAVIOR DURING CARDIAC ARRHYTHMIAS
}

\author{
By \\ Mark-Anthony P. Bray \\ Dissertation \\ Submitted to the Faculty of the \\ Graduate School of Vanderbilt University \\ in partial fulfillment of the requirements \\ for the degree of
}

DOCTOR OF PHILOSOPHY

in

Biomedical Engineering

May, 2003

Nashville, Tennessee

Approved by:

Professor John P. Wikswo

Professor Shien-Fong Lin

Professor Robert L. Galloway

Professor Robert J. Roselli

Professor Richard G. Shiavi 


\section{ACKNOWLEDGMENTS}

Needless to say, this dissertation could not have been completed without help from a number of people.

First of all, I'd like to thank John Wikswo for his mentorship during my time here. I've appreciated the fact that he made his intentions for me very clear from the get-go: to train me to do science. His personal intensity towards his pursuit of research has been admirable. Even more importantly, he has made himself accessible enough such that we could form a personal rapport beyond simply a professional working relationship; I consider it a milestone when I was first able to honestly display my displeasure towards a particular task. By being willing to converse about my thoughts concerning my work, whether negative or positive, not only was I better able to exchange ideas but I also knew he cared about what I thought as a person. The times when we have been able to chat about non-work-related topics have also been meaningful to me. For these reasons, it is my sincere hope that we will be able to maintain contact once I leave Vanderbilt for parts unknown.

I would like to give special thanks to Bob Galloway, not only for being on my committee, but also for his willingness to accommodate me in his lab space. I've often commented to people how odd it can be to inhabit an office that has nothing to do with my research whatsoever, but even so, I am grateful to him for granting me the elbow room in which to work. Not to mention his good-naturedness and humor, which has been an encouragement to me.

In much the same vein, I'm also grateful to the rest of the SNARL lab, both past and present. In particular, I want to mention Tuhin Sinha, Dave Cash, Chad Washington, Prashanth Dumpuri and especially Andy Bass. Again, my lab-mates have (frequently) pointed out the same sentiment about being in Dr. Galloway's space that I expressed above. But joking aside, they have always made me feel at home there. Even as my research has diverged more and more from the lab's research, my friends there have stayed the same. I've recognize how rare it can be to have the camaraderie that we have in SNARL; there's never a time when I can't talk to my labmates about serious matters, humorous matters, and everything in-between. Not only has it been edifying for me, but it's been more fun than I have any right to expect, and I truly regret have to leave a place with such people in it. 
Support within the department has also come from Jean Alley and Dr. Cynthia Paschal, my upper-echelon cheering section. I sill remember when Cynthia was kind enough to give me a cupcake with a candle in it when my qualifying exam had the unfortunate timing to fall on my birthday. Jean and I have had more lunches together than I have had with anyone else in the department. Both of them have been open doors when it comes to talking about issues about life and other matters of consequence, and they have been my sisters in Christ within BME.

I'd like to thank Steve Hartmann, my erstwhile office-mate. He had the good fortune of graduating a year before me (nothing like having a baby to motivate you), but his work ethic has been of immense help in keeping me on task. It certainly has not hurt to have a sense of friendly rivalry in place to egg me on. Also, it has been a joy to vicariously experience the wonders of fatherhood through him; I hope at some point to be the type of doting parent that he has become.

I also thank my current office-mate Steve Gebhart (in accordance with the Conservation of Steve Rule: Steves can neither be created nor destroyed but only replaced, especially if they're my office-mate). Much like Steve \#1, he has helped create an environment conducive to work, and has made it a pleasure to come in each morning. His friendship has been much appreciated.

Marcella Woods deserves special mention. Even though I knew her back at Tulane, I have gotten to know her much better in the last few years here. Since we share the same advisor, there's always ample opportunity to commiserate about what's going on with our work. I have come to admire her perseverance and her resilience in the face of setbacks that most likely would have made most grad students crumble. I am grateful and indebted to her for her friendship and time.

The people of Graduate Christian Fellowship have been more valuable than I can possibly imagine. Through GCF I have been able to meet more people with more diverse backgrounds than at any other point, people who I would never have met any other way. With them I have grown as an individual and as part of a community, and they have shared their time, and indeed themselves, with me almost every step of the way. I thank them for their kindness, their compassion, their grace above all, and most importantly, helping me to stay focused on those matters of ultimate importance.

Of course, I certainly cannot forget my sister Melody and my brother Mike. Both have been willing to contact and check up on me during my sometimes long periods of silence. My parents have been a constant encouragement to me, even as they asked at least once a year, 
"Explain to me again, exactly what is it that you do?" I know my dad is proud of me. I know my mom has been praying for me since Day One. I thank them both for their faithfulness and consistency.

And finally, the LORD my God, by whom I live and have my being, thank you. You have promised abundant life and I have experienced it first-hand. You have been my one comfort, and I will continue to follow You.

S.D.G. (Soli Deo Gloria) 


\section{TABLE OF CONTENTS}

Page

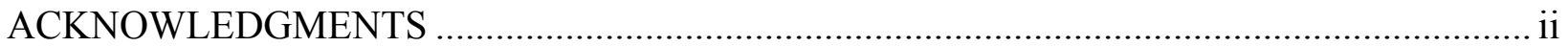

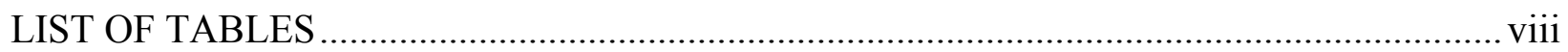

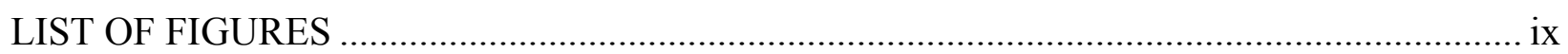

Chapter

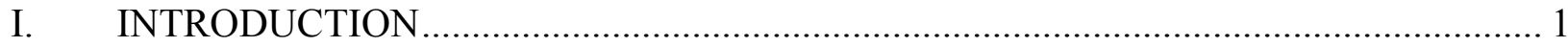

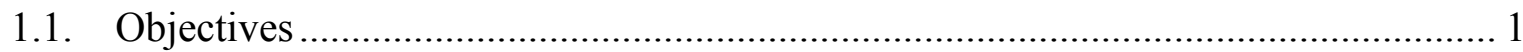

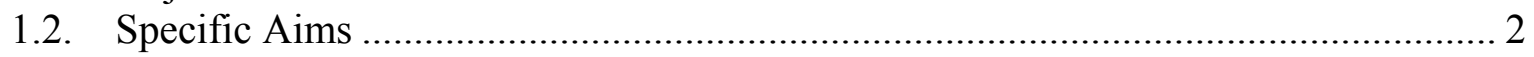

II. BACKGROUND AND SIGNIFICANCE ............................................................ 4

2.1. Introduction: Significance of ventricular fibrillation and defibrillation................... 4

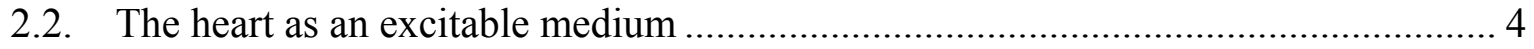

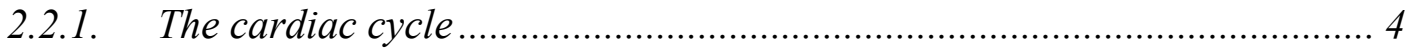

2.2.2. Details of cardiac electrical properties ................................................. 5

2.2.3. Models of cardiac electrical and structural properties ............................ 7

2.3. Reentry as a mechanism for fibrillation .................................................... 9

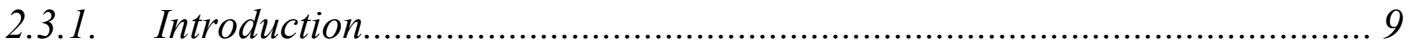

2.3.2. Anatomic Reentry ............................................................................... 10

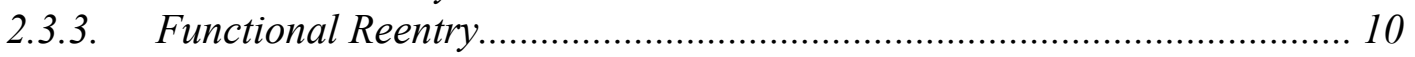

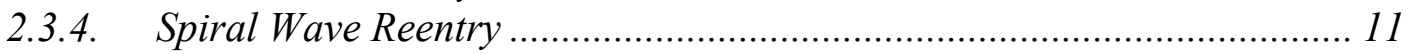

2.3.5. Spiral Wave Motion .......................................................................... 15

2.3.6. Three-dimensional spiral waves .................................................... 16

2.3.7. Scroll waves and ventricular arrhythmias .......................................... 17

2.4. Phase portrait analysis ................................................................................... 19

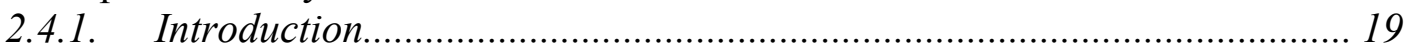

2.4.2. Applications to cardiac models...................................................... 19

2.5. Phase singularities and filaments as topological defects ................................... 22

2.6. Cardiac optical mapping ................................................................................. 24

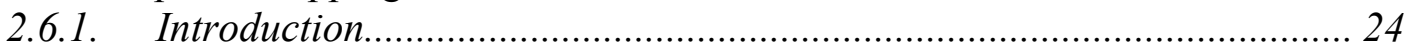

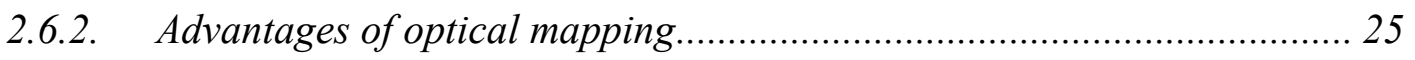

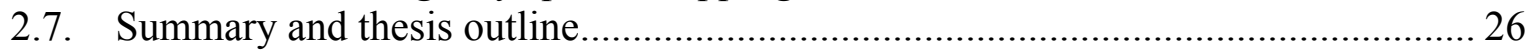

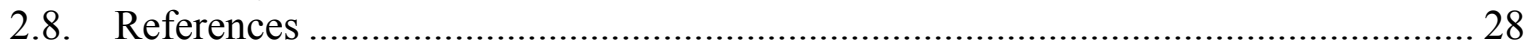

III. EXPERIMENTAL AND THEORETICAL ANALYSIS OF PHASE SINGULARITY DYNAMICS IN CARDIAC TISSUE................................................................. 38

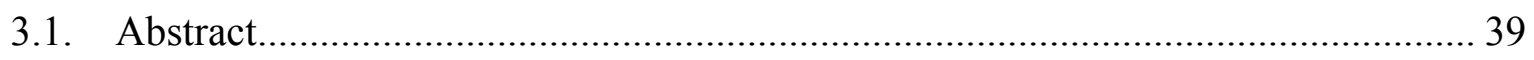

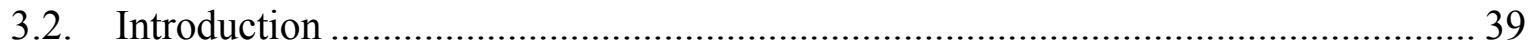

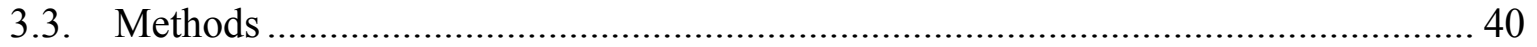

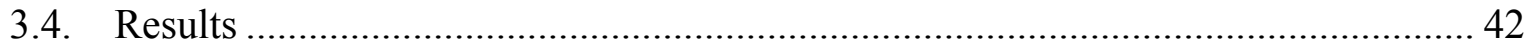

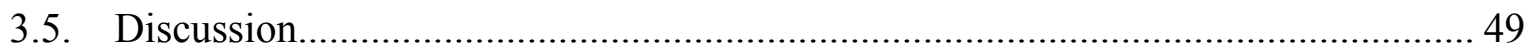




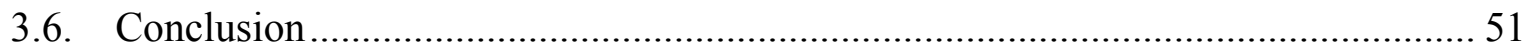

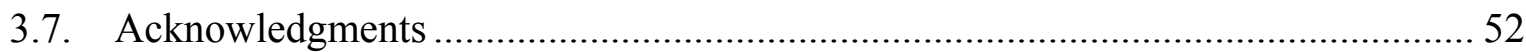

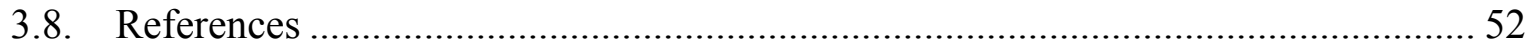

IV. CONSIDERATIONS IN PHASE PLANE ANALYSIS FOR NON-STATIONARY

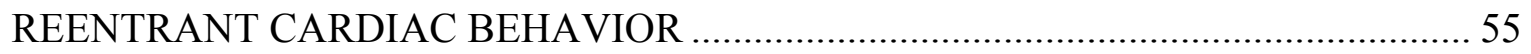

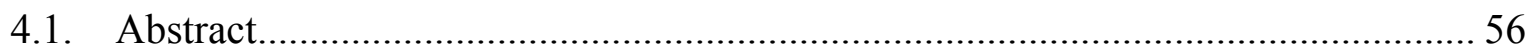

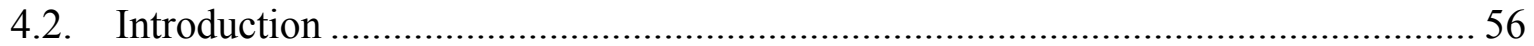

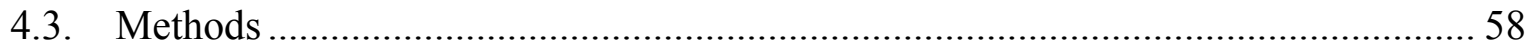

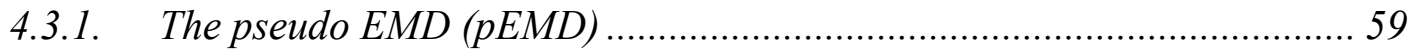

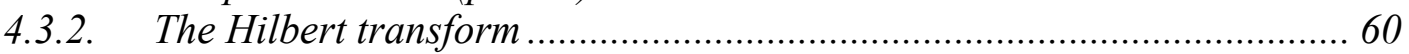

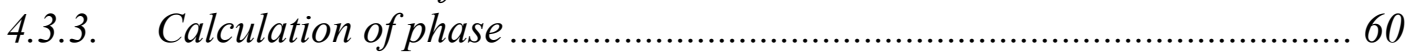

4.3.4. Numerical simulation and experimental preparation.............................. 60

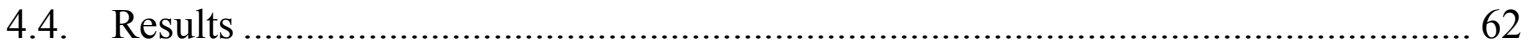

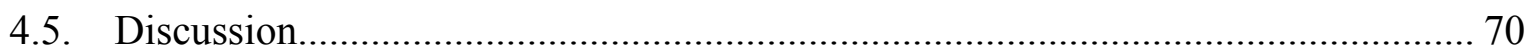

4.5.1. Observation of Initial Singularity Formation ......................................... 70

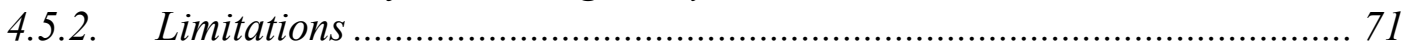

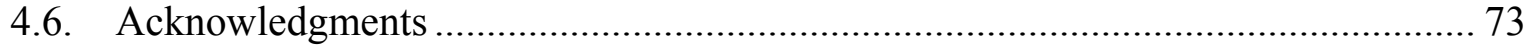

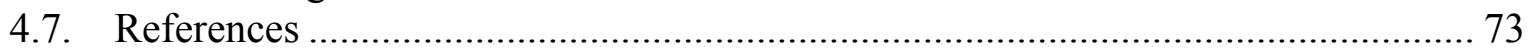

V. USE OF TOPOLOGICAL CHARGE TO DETERMINE FILAMENT LOCATION IN A NUMERICAL MODEL OF SCROLL WAVE ACTIVITY …………………….... 75

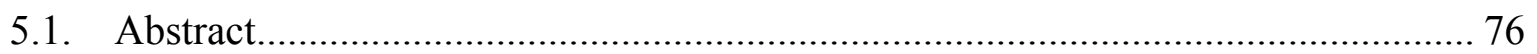

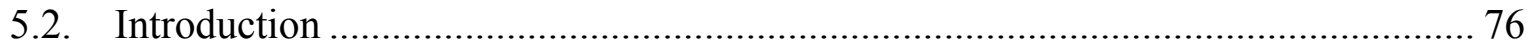

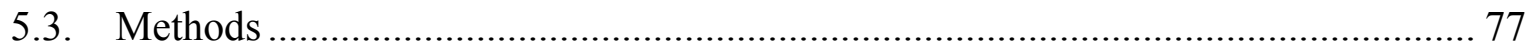

5.3.1. Two-variable reaction-diffusion model.................................................. 77

5.3.2. Two-variable state space and phase …………….................................. 77

5.3.3. Time-series reconstructed state space ………..................................... 78

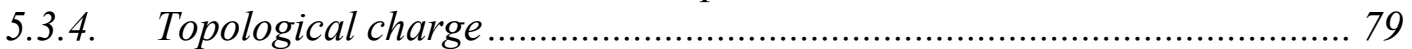

5.3.5. Computation of topological charge as a convolution operation ............... 79

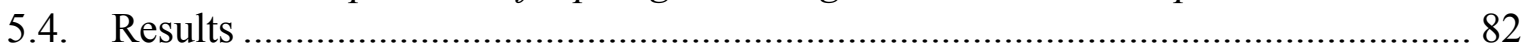

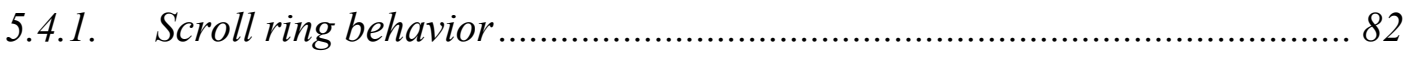

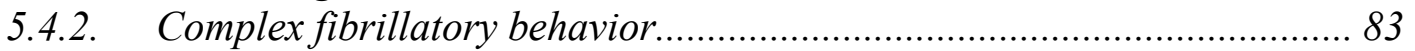

5.4.3. Comparison between two-variable and time-series state space ............... 83

5.4.4. Three-variable reaction-diffusion model .............................................. 88

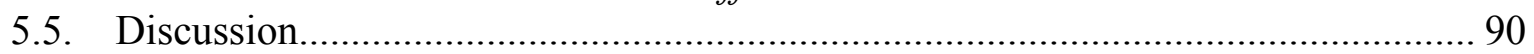

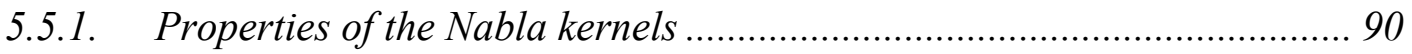

5.5.2. The issue of state space selection...................................................... 91

5.5.3. Future directions........................................................................... 92

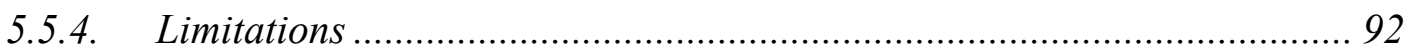

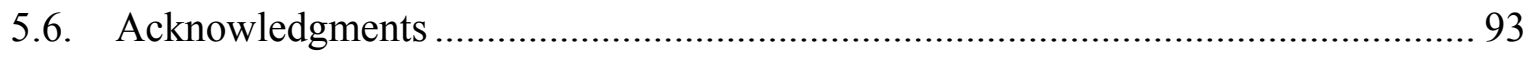

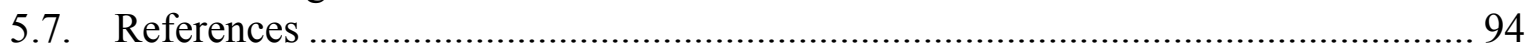

VI. BIFURCATIONS AND AN INTERACTION POTENTIAL IN DECAYING

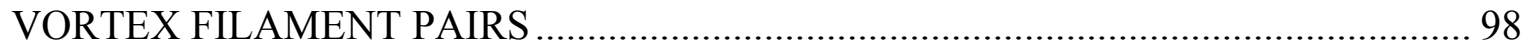

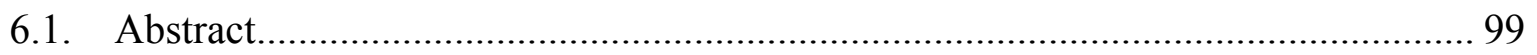

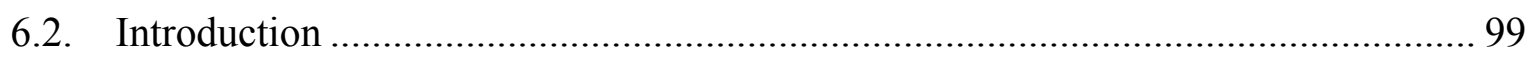




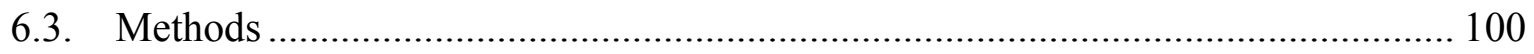

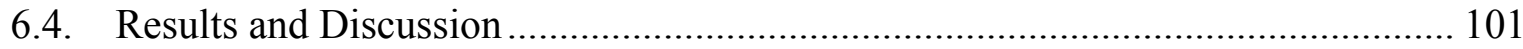

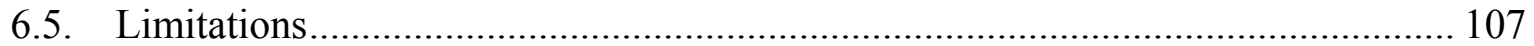

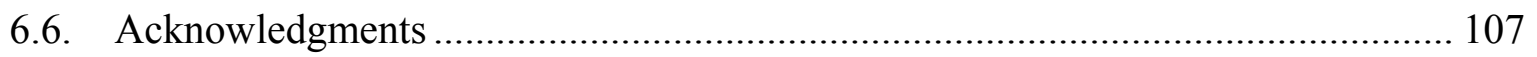

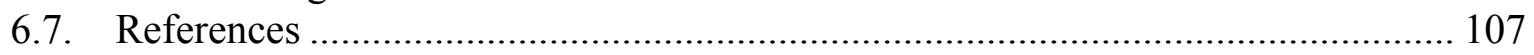

VII. EXAMINATION OF OPTICAL DEPTH EFFECTS ON FLUORESCENT

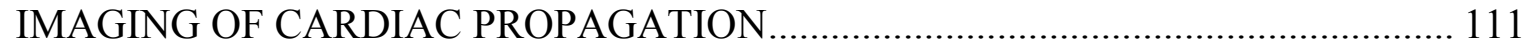

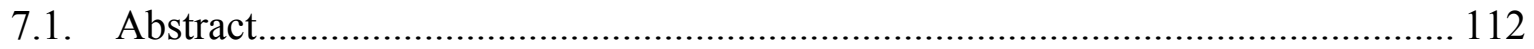

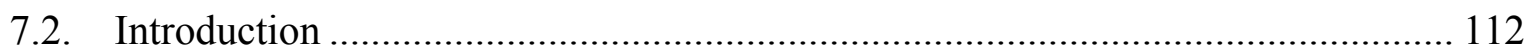

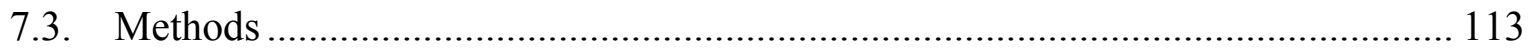

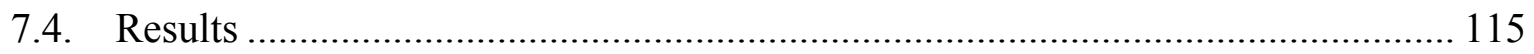

7.4.1. Cross-section of intramural filament ............................................... 115

7.4.2. 3-D elliptical filament, no fiber rotation............................................. 122

7.4.3. 3-D scroll wave breakup in presence of fiber rotation ........................... 123

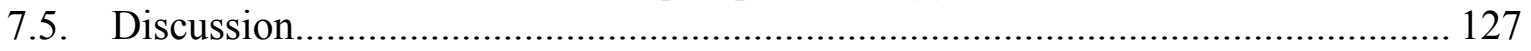

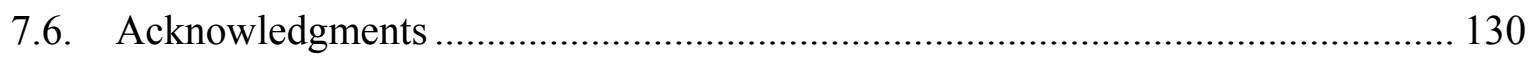

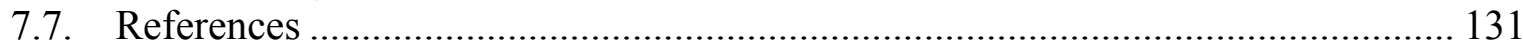

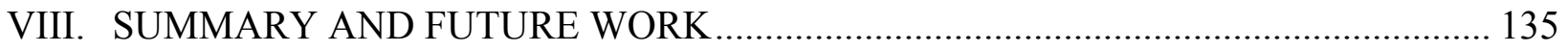

8.1. Limitations and future work ……………………............................................ 136

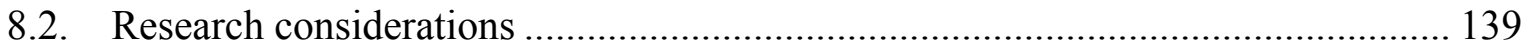

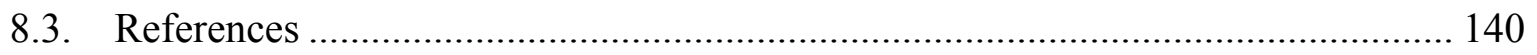

Appendix

A. THREE-DIMENSIONAL VISUALIZATION OF PHASE SINGULARITIES

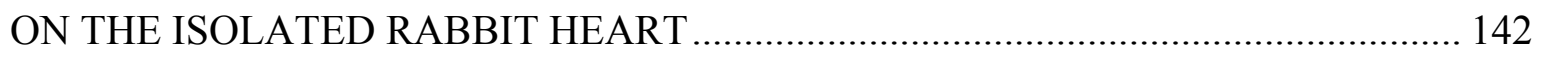

A.1 Introduction: Whole-heart optical mapping …………....................................... 143

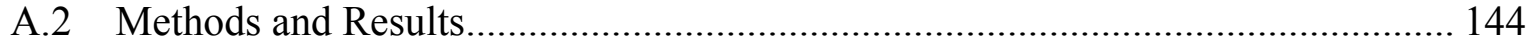

A.3 Acknowledgments ................................................................................... 145

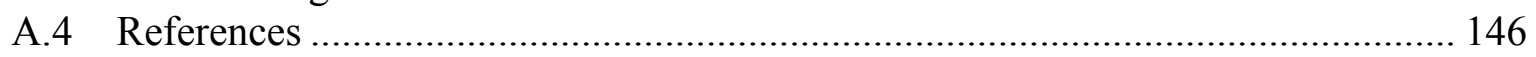




\section{LiST OF TABLES}

Table

Page

5.1: Measured time-of-life and error between wavetip trajectories on the top surface produced by the two-variable state space $(2 \mathrm{~V})$ and time-series state space reconstruction (TS). Time-of-life measured in time units (t.u.), error measured in space units (s.u.). ....... 87 


\section{LIST OF FIGURES}

2.1: Illustration of a typical ventricular action potential................................................. 6

2.2: Diagram of the core conductor model with circuit segments of length $d x$....................... 8

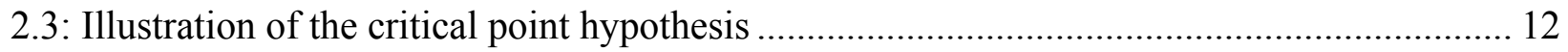

2.4: Creation of (A) single spiral and (B) figure-of-eight reentry via cross-field stimulation...... 13

2.5: (A) Illustration of the critical point hypothesis in quatrefoil reentry for cathode break S2 stimulation. (B) Creation of quatrefoil reentry in a Beeler-Reuter model ........................ 14

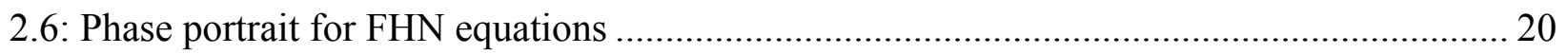

2.7: Examples of phase portraits for the BZ two-variable $(u, v)$ model.................................... 21

3.1: Images of the spatiotemporal distribution during quatrefoil reentry ................................ 43

3.2: Depiction of the spatiotemporal evolution of a quatrefoil reentrant wavefront pattern relative to regions of low variance of wavefront amplitude.

3.3: Numerically simulated (left column) and experimental (right column) quatrefoil reentry following an electrical stimulus applied to the center of tissue .................................. 46

3.4: Demonstration of trajectories of quatrefoil phase singularities in $(x, y, t)$ space, illustrating dynamic behavior in time.

3.5: Plots of the average of the TP and LP separation distances for all instances of quatrefoil reentry grouped into the three types

3.6: Taxonomy of the pair separation distance $\mathrm{S}$ (in $\mathrm{mm}$ ) plotted as a function of time $\mathrm{T}$, computed as a fraction of the rotation period

3.7: Cross-correlation of TP and LP separation distances

3.8: Depiction of the spatiotemporal evolution of a single spiral wave from Fig. 3.2 relative to region of low variance of wavefront amplitude and phase singularity trajectory....

4.1: (A) An isochronal map from numerically simulated data. (B) Transmembrane signal measured at site indicated by the black arrow in (A). (C) Phase portrait of the signal shown in (B) using $\tau=3$.

4.2: Example of phase space trajectories resulting from poor choices of $\tau$.......................... 57

4.3: Flowchart of the algorithm as applied to numerically simulated data.............................. 61

4.4: (A) Example of phase trajectory using Hilbert transform. (B) Expanded view of (A) illustrating the highlighted segment.

4.5: Comparison of phase maps calculated from numerically simulated data.

4.6: Comparison of phase singularity maps generated from various values of $\tau$ and the pEMD-Hilbert transform

4.7: (A) An isochronal map from experimental data. (B) Transmembrane signal measured at site indicated by arrow in (A). (C) Phase portrait of the signal shown in (B) using $\tau=7 \ldots .65$ 
4.8: Flowchart of the algorithm as applied to experimental data

4.9: (A) Example of phase trajectory using pEMD-Hilbert transform. (B) Expanded view of (A) illustrating the highlighted segment.

4.10: Comparison of phase maps calculated from the experimental data set used to obtain Figs. 4.7-4.9..

4.11: Phase singularity maps during S2 stimulation using experimental data using $\tau=7$ (left column) and the pEMD-Hilbert transform (right column)....

4.12: (A) $V(t)$ traces from point located within virtual cathode (solid black curve) and virtual anode (solid blue curve) (B) $V^{\prime}(t)$ traces corresponding to $V(t)$ in (A) .

4.13: Phase trajectories for the data at the virtual cathode in Fig. 4.12 with the segment corresponding to the duration of S2 stimulus drawn in blue

5.1: (A) A 2-D cross-section of a scroll wave mapped in the state space. (B) The position of the numbered pixels in the phase portrait.

5.2: Trajectories in state space from all elements of a 2-D grid containing a spiral wave generated using the system described by Eq. (5.10) and (5.11).

5.3: (A) Snapshots of a cross-section through a simulated vortex ring. (B) Snapshots during the spontaneous annihilation of the vortex ring.

5.4: Trajectories in state space from all elements of a 2-D grid containing a spiral wave generated using the system described by Eq. (5.12).

5.5: Three-dimensional snapshots of vortex filaments generated by the system described by Eq. (5.12) during unstable scroll wave reentry

5.6: Phase portraits generated by the system described by Eq. (5.12) for (A) two variable state space and (B) time-series state space. $\tau$ is set at 1.26 t.u in (B).

5.7: (A) An example of the waveforms $v(t), u(t)$, and $w(t)$ produced by the system described by Eq (5.13). (B) $(u(t), v(t))$ state space. (C) $(u(t), w(t))$ state space. (D) $(w(t), v(t))$ state space.

5.8: Wavetip path traced out by the system described by Eq. (5.14) for $(u(t), v(t))$ and $(u(t), w(t))$ state spaces.

6.1: Quatrefoil reentry produced by cathodal break (left column) and anodal break (right column)

6.2: (A) Scroll-ring lifetime $T_{L}$ as a function of $\left(R_{0}, Z_{0}\right)$ in cathodal break simulation.

(B) Initial trajectories in the $(\rho, z)$ plane. (C) Relationship between the initial separation distance $\left(Z_{0}\right)$ and initial scroll rings velocity in the $z$-direction $\left(V_{0(z)}\right)$.....

6.3: (A) Scroll-ring lifetime $T_{L}$ as a function of $\left(R_{0}, Z_{0}\right)$ in anodal break simulation.

(B) Initial trajectories in the $(\rho, z)$ plane. (C) Relationship between the initial separation distance $\left(Z_{0}\right)$ and initial scroll rings velocity in the $z$ direction $\left(V_{O(z)}\right)$.....

7.1: (A) The three-dimensional model (left) and the cross-section AA' (right) showing the position of the singularity at $t=0 \mathrm{~ms}$ (open circle) and the two recording locations on the 
epicardium (1 and 2). Time traces at point (1) are shown in (B); time traces at point (2) are

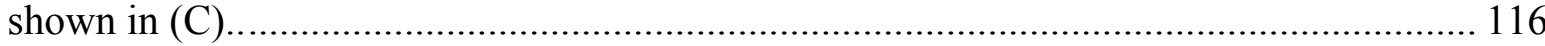

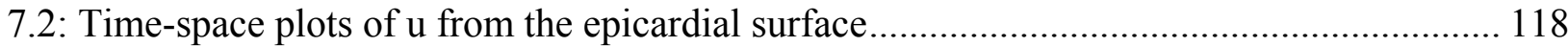

7.3: Snapshots of the temporal development of the depth-weighted and top layer signals........ 119

7.4: Snapshots of the temporal development of the depth-weighted and top layer signals

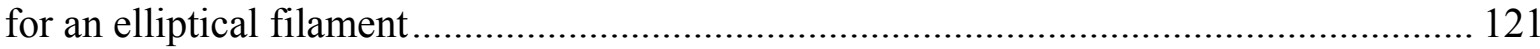

7.5: Comparison of $\bar{u}$ and $u_{0}$ signals during spiral wave breakup.......................................... 123

7.6: Illustration of phase singularity localization during a 20-ms segment of fibrillatory

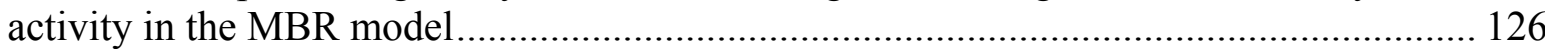

A.1: Whole-heart geometric reconstructions mapped with (A) temporal derivative of

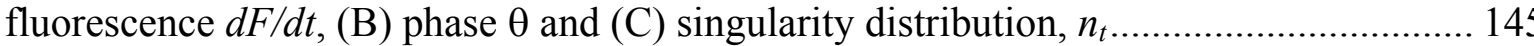




\section{CHAPTER I}

\section{INTRODUCTION}

\subsection{Objectives}

The objectives of this research were the development and utilization of new techniques to analyze optical recordings of cardiac spiral wave activity, both in the context of local electrodynamic behavior and global large-scale behavior. Optical imaging is an emergent technique which records changes in the cardiac transmembrane potential by the use of voltagesensitive fluorescent dyes. This approach permits precise, non-invasive measurement of the transmembrane potential with high spatial and temporal resolution.

It is well known that the development of unstable, self-sustaining reentrant waves of electrical activity underlies life-threatening arrhythmias such as ventricular tachycardia and fibrillation. However, many details regarding the mechanisms of reentry remain unclear. Recent work has shown that fibrillation exhibits a large amount of spatiotemporal organization. An understanding of the response of cardiac tissue to an electrical stimulus is crucial for proper treatment and prevention of cardiac arrhythmias; an example is the application of a strong defibrillating shock to halt fibrillation. Optical imaging has played a key role in discerning the spatiotemporal patterns of fibrillation and in the verification of theoretical computational models of cardiac propagation.

Application of this imaging modality has provided a new and promising form of analysis: cardiac activation may be quantified by plotting the transmembrane potential signal against a time-delayed version of itself to form a phase portrait. This procedure allows the conversion of images of voltage into images of the phase of the tissue during the cardiac cycle and as a result provides a means to detect phase singularities. Since phase singularities are believed to be the organizing centers to reentrant activity, a reliable method of isolating the singularities from the

optical image not only permits analysis of the salient features of fibrillation but also reduces the information content necessary to track their motion and interaction.

The goal of this project was to apply novel techniques of data visualization and phase portrait analysis to explore the dynamics of spiral wave formation and interaction and the 
electrical activity evident at the epicardial surface as well as within the underlying myocardial substrate.

\subsection{Specific Aims}

The following specific aims were necessary to accomplish the objectives of this research:

Aim 1. Implement an algorithm to localize phase singularities based upon the phase portrait calculation. A phase portrait of the cardiac system (derived either from experimentally obtained or numerically simulated data) may be used to generate a phase map. The concept of topological charge was applied to the phase map to provide a mathematically robust means of isolating the phase singularity. This technique was then used to perform an experimental and theoretical analysis of phase singularity interaction dynamics.

Aim 2. Improve the characterization of the cardiac phase portrait. While the use of the timeseries analytical method is a standard means of reconstructing the phase portrait from a single variable, some shortcomings remain. This aim explored a re-definition of the phase portrait for the purpose of overcoming these limitations and optimizing the use of the phase calculation in order to capture essential elements of singularity initiation.

Aim 3. Extend the algorithm developed in Specific Aim 1 to filament localization. Filaments are the three-dimensional analogue of the phase singularity, and are believed to underlie fibrillatory activity in the context of the three-dimensional myocardium. The algorithm developed earlier was extended to isolating the filament in numerical simulations. Considerations which govern the choice of variables for a phase portrait derived from a multi-variable system were also examined.

Aim 4. Examine the interaction behavior of a filament pair. Early numerical predictions of singularity interactions exhibited morphological differences from the experimental observations, indicating that more sophisticated numerical models are required. Since the singularity dynamics are intricately tied to underlying filament interactions, a simple model was created to study the interaction dynamics of a pair of adjacent circular filaments. This behavior was quantified in terms of the filament time-of-life as a function of the initial separation and radius of the filament pair.

Aim 5. Quantify the effects of the depth-weighted optical signal on epicardial propagation behavior. Studies indicate that the location of filaments may be deduced on the basis of 
the behavior of transmembrane potentials recorded on the epicardium, presumably due to the fact that the optically recorded signal is depth-dependent. For this aim, a threedimensional cardiac model was created to simulate filament behavior. The corresponding phase singularity and epicardial potential behavior was examined both with and without the inclusion of a weighted optical signal.

Aim 6. Further substantiate the results of Specific Aims 1 and 2 by performing three-dimensional whole-heart epicardial visualization. Optical imaging techniques are applied traditionally to a single view that presents the potential distribution of a fraction of the cardiac surface, which is a limitation when examination of whole-heart phenomena is desired. Recently, a novel panoramic imaging algorithm was developed and validated, and hence provided a proof-of-concept that this obstacle can be surmounted. This aim was concerned with the practical application of this visualization technique to extend our nonlinear analysis to the full epicardial surface. 


\section{CHAPTER II}

\section{BACKGROUND AND SignificANCE}

\subsection{Introduction: Significance of ventricular fibrillation and defibrillation}

Ventricular fibrillation, a phenomenon characterized by rapid, uncoordinated electrical and mechanical activity without effective ventricular contraction and cardiac output, is the most common cause of sudden cardiac death in the industrialized world, with nearly a quarter of all human deaths attributable to this pathology [1]. The basic mechanisms by which fibrillation are initiated are not fully understood. Defibrillation, a process that halts fibrillation, may be performed chemically or mechanically but is most commonly accomplished by the application of a strong electric potential across the heart. Despite the fact that the life-saving effects of defibrillation have been known for close to a century [2], the mechanisms by which electrical defibrillation achieves its effects are not fully understood. The future design and application of clinical arrhythmia treatments such as pacemakers and defibrillators is dependent upon our understanding of both these phenomena. What is clear, however, is that the best approach to obtain the necessary information for understanding both fibrillation and defibrillation is to study the distribution of potentials over both the entire heart and individual cardiac myocytes. Since electrical stimuli are thought to alter the transmembrane potential to generate cellular excitation, it is specifically the spatiotemporal distribution of the transmembrane potential $\left(V_{m}\right)$ during fibrillation and defibrillation that is under intense scrutiny in cardiac electrophysiology. Given this specification, the question remains of identifying how arrhythmias manifest themselves in the heart and what techniques are available to a researcher who wishes to examine this phenomenon.

\subsection{The heart as an excitable medium}

\subsubsection{The cardiac cycle}

At the most basic level, the heart is an electromechanical organ which functions to supply the metabolic needs of the organism by pumping blood to and from all necessary tissues. This task is performed by means of a coordinated muscular contraction cycle which progresses across 
the four chambers of the heart: the right atria (RA), left atria (LA), the right ventricle (RV) and left ventricle (LV). This mechanical process is mediated by the underlying electrical properties of the cardiac tissue, i.e., coordination of the contractile behavior is dependent upon reliable coordination of a series of electrical signals and pathways. The basic cardiac cycle is as follows:

(a) Deoxygenated blood fills the RA from the superior and inferior vena cava. Meanwhile, newly oxygenated blood from the lungs enters the LA. The sinoatrial node (SAN) located in the RA initiates the electrical cycle by firing an electrical pulse. This wave sweeps across both atria and causes the RA and LA to deposit their blood in the RV and LV, respectively.

(b) The atria are electrically isolated from the ventricles except at one point, the atrioventricular node (AVN). Once the wave from the SAN reaches the AVN, the AVN fires and a propagating wave is transmitted down a specialized conducting system (the bundle of His and the Purkinje fibers) located in the septal wall dividing the RV and LV.

(c) This wave is then distributed to the ventricular wall and propagates to the surface of the heart. This results in a synchronized contraction of the ventricles; the RV pushes the deoxygenated blood towards the lungs through the pulmonary artery for oxygenation and the LV pushes the oxygenated blood out to the rest of the body through the aorta.

It is straightforward to see that a failure of the electrical conduction system leads to a breakdown in the rhythm of muscular contraction, with often fatal results if left unremedied.

\subsubsection{Details of cardiac electrical properties}

The nature of the propagating electrical wave (known as an action potential) is mediated by ion channels embedded within the cardiac cellular membrane. The cardiac membrane is a member of a class of cells with excitable properties, because the application of a sufficiently large external stimulus will cause the voltage across the cell membrane (the transmembrane potential, $V_{m}$ ) to undergo a large excursion in magnitude before returning to the resting potential. Furthermore, the diffusive coupling between the cells permits the excitatory wave to be transmitted from one cell to its neighbors without attenuation with distance from the starting cell. These general properties are common to a set of systems known as excitable media and can be observed in many chemical and physical systems, as well as in other areas of biology [3].

For the most part, the most important channels are those that conduct sodium $\left(\mathrm{Na}^{+}\right)$, potassium $\left(\mathrm{K}^{+}\right)$and calcium $\left(\mathrm{Ca}^{2+}\right)$; each of these are comprised of gating proteins which open 


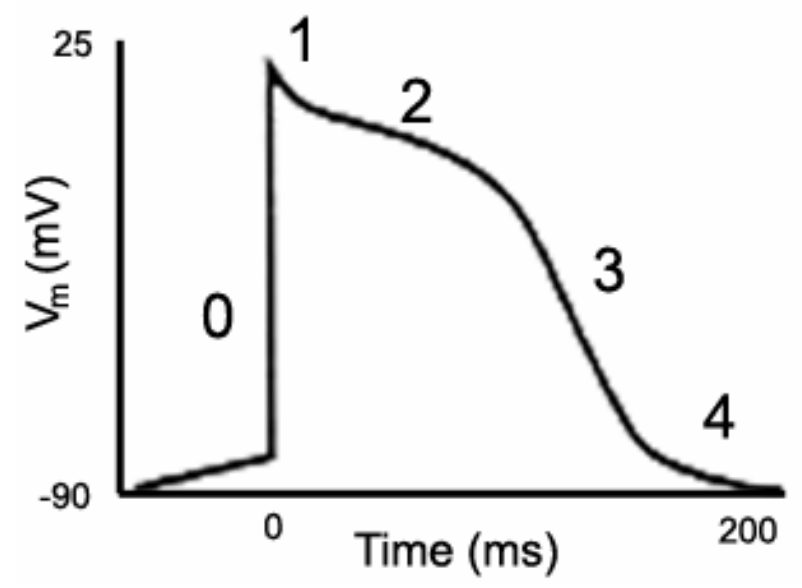

Figure 2.1: Illustration of a typical ventricular action potential. Phases are labeled along the graph.

and close in response to the transmembrane voltage experienced across the channel and with a time constant associated with the transition of the protein from an open to a closed state. In the resting state, a ventricular cardiac cell maintains a $V_{m}$ value of about $-90 \mathrm{mV}$ until a suprathreshold stimulus is applied and triggers an action potential. The cardiac action potential progresses through the following phases (several of the fluxes are mediated by channels of multiple types so only the major ions are described here):

(a) Phase 0: A change in $V_{m}$ (usually induced by neighboring cells) causes the opening of voltage-gated fast $\mathrm{Na}^{+}$channels. The influx of sodium results in a depolarizing reversal in $V_{m}$ from -90 to $+25 \mathrm{mV}$. At the peak of the action potential, the $\mathrm{Na}^{+}$channels inactivate.

(b) Phase 1: A transient outward $\mathrm{K}^{+}$current causes a small early decrease in $V_{m}$.

(c) Phase 2: The depolarization causes $\mathrm{Ca}^{2+}$ channels to open, permitting a calcium influx from both extracellular sources and from the sarcoplasmic reticulum. At the same time, an efflux of $K^{+}$begins. The balance between these two currents produces a plateau in the action potential shape and is responsible for the action potential duration typically being on the order of 200-300 ms. Contractile activity also occurs during this period due to the presence of $\mathrm{Ca}^{2+}$.

(d) Phase 3: As the $\mathrm{Ca}^{2+}$ channels close, additional slow $\mathrm{K}^{+}$channels open; their efflux results in repolarization.

(e) Phase 4: The final ion concentrations are restored via ion exchangers and ATPase resulting in $V_{m}$ returning to rest. 


\subsubsection{Models of cardiac electrical and structural properties}

The pioneering work of Hodgkin and Huxley in creating a quantitative model of the propagating electrical behavior of a squid giant axon by decomposing it into a set of principle ionic currents has provided the impetus of the production of a wide variety of similar models tailored for the cardiac membrane [4]. Examples of active membrane models range from two variable systems (FitzHugh-Nagumo [5]) to four (Beeler-Reuter [6]) to ten (DiFrancesco-Noble [7]) in order to replicate the basic current components or ensembles of currents, to more sophisticated and physiologically realistic models with in excess of fifteen variables (Luo-Rudy II [8] and its successors). Since there are a variety of excitable cellular types in the mammalian heart, the design of action potential models must be tailored for the specific tissue in question. In addition, a balance must be struck between avoiding computational complexity and desiring to specify as many ionic pathways in as much detail as possible. As additional research reveals new currents and refines measurements of known currents and ion concentrations, even more complex (and hopefully more realistic) models will be possible.

Regardless of the active kinetic model chosen, the membrane model must then be combined with a representation of the underlying spatial domain in which these fluxes act. For excitable media in general, this coupling is described in the form of a reaction-diffusion equation applied to a scalar field of concentrations $u(\vec{r}, t)$ in the following generalized form:

$$
\frac{\partial u}{\partial t}=\nabla \cdot(D \nabla u)+F(u),
$$

where $\vec{r}$ is the position vector, $t$ is time, $D$ is the diffusion coefficient tensor and $F$ is a function of $u$ (often non-linear) containing the mass-action law terms.

As a first approximation, the cardiac fiber may be modeled as an axially symmetric onedimensional cable composed of a membrane separating an extracellular space from the intracellular space. In this system, only the current across the membrane (transmembrane current) proceeds radially; all other currents are axial. Furthermore, the axial currents are ohmic,

allowing traditional circuit theory to be applied to the problem; specifically, the intracellular and extracellular currents have intracellular and extracellular resistances $r_{i}$ and $r_{e}$ associated with them. This set of initial assumptions comprises what is known the core conductor model, which is illustrated in Fig. 2.2 [9]. 


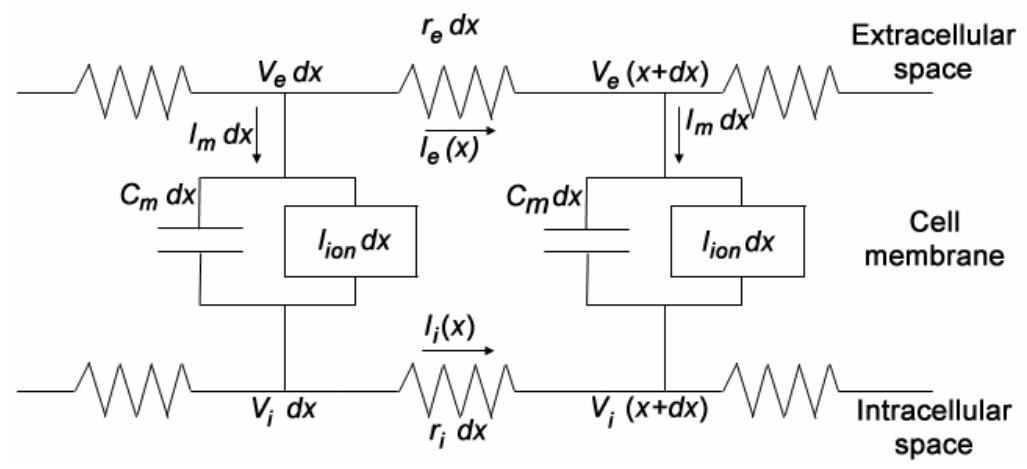

Figure 2.2: Diagram of the core conductor model with circuit segments of length $d x$.

Lastly, it is assumed that the transmembrane current is the sum of capacitive and ionic currents; that is,

$$
I_{m}=C_{m} \frac{d V_{m}}{d t}+I_{i o n}
$$

where $I_{m}$ is the current density, $C_{m}$ is the specific membrane capacitance, $I_{i o n}$ is the contribution from the ionic fluxes and $V_{m}$ is the transmembrane potential. By recognizing that any change in the axial current over a distance $d x$ must be equal and opposite to a change in $I_{m}$ by virtue of conservation of current, the following formula is obtained,

$$
\frac{1}{p\left(r_{e}+r_{i}\right)} \frac{\partial^{2} V_{m}}{\partial x^{2}}=C_{m} \frac{\partial V_{m}}{\partial t}+I_{i o n}
$$

where $p$ is the circumference of the cable. By appropriate nondimensionalization of Eq. (2.3), one can obtain an result of the same form as (2.1).

In reality, cardiac cells are connected together via gap junctions which allows current to flow from cell to cell within the intracellular medium without crossing the membrane into the surrounding extracellular space. Since $V_{m}$ reflects the voltage present in both domains at a given time, a model is required that can describe the complexity of the interconnectivity between the intracellular and extracellular regions. Such a spatial representation was introduced by Tung in which the structural geometry of the interconnection between the two domains is homogenized to produce a pair of coupled equations [10]

$$
\begin{gathered}
\nabla \cdot\left(\tilde{\sigma}_{i} \nabla V_{i}\right)=\beta I_{m}, \\
\nabla \cdot\left(\tilde{\sigma}_{e} \nabla V_{e}\right)=-\beta I_{m},
\end{gathered}
$$


where $\sigma_{i}$ and $\sigma_{e}$ are the electrical conductivity tensors and $\beta$ is the surface area-to-volume ratio; these quantities are spatially averaged such that the system is homogeneous. The membrane current density $I_{m}$ is the same as in Eq. (2.2) and $V_{i}$ and $V_{e}$ are the intracellular and extracellular voltages such that $V_{m}$ is $V_{i}-V_{e}$. It should be noted that the two domains share the same space, and both equations must be solved simultaneously at each spatial point. Essentially, Eqs. (2.4) and (2.5) describe a system in which the total current is conserved between the two domains, with the cellular membrane as the only route by which the current can cross. If the conductivity tensors in the two domains are proportional, i.e., $\tilde{\sigma}_{i}=k \tilde{\sigma}_{e}$ where $k$ is a constant (equal anisotropy), Eqs. (2.4) and (2.5) collapse into a form of (2.3), also known in this context as a monodomain equation. In reality, the ratios of conductivity values longitudinal and transverse to the fibers in the intracellular and extracellular domains are generally unequal (in other words, $\left.\sigma_{i(\text { long })} / \sigma_{i(\text { trans })} \neq \sigma_{e(\text { long })} / \sigma_{e(\text { trans })}\right)[11]$. This unequal anisotropic character of the cardiac tissue leads to a number of interesting, even surprising, theoretical predictions of this model with respect to the response of the tissue to an externally applied stimulus, many of which have been confirmed by experiment $[12,13]$. For this reason, the bidomain system is generally regarded as the current state-of-the-art in terms of modeling the cardiac excitable medium [14].

\subsection{Reentry as a mechanism for fibrillation}

\subsubsection{Introduction}

The electrical properties mentioned above allow for much more complex patterns of propagating waves than the simple planar propagation representative of the cardiac cycle, provided the proper conditions are present. This electrical irregularity, termed an arrhythmia, can usually manifest itself in one of two ways: (1) tachycardia, characterized by excessive periodic electrical activity (this category is further subdivided into monomorphic if one frequency is present, and polymorphic if more frequencies are present); and fibrillation, characterized by more irregular and disorganized electrical activity. In general, although both arrhythmias can occur on the atria, they are usually not considered to be life-threatening. However, ventricular fibrillation is much more serious since the primary contractile action of the heart is disrupted. Furthermore, it is not uncommon for ventricular tachycardia to degenerate into ventricular 
fibrillation. The hypothesis of fibrillation occurring by means of uneven and repetitive electrical propagation was first proposed over a century ago [15]. Based on continuing research, the phenomenon of reentrant excitation, in which a wave of excitation repeatedly activates the same area of tissue independently of the natural cardiac rhythm, is believed to play a significant role in the initiation of such lethal arrhythmias [16].

\subsubsection{Anatomic Reentry}

The physical features of the heart, such as the arteries and connective tissue bundles, combined with the various tissue types and their associated differing electrophysiologic properties, may form the anatomical basis for the formation of reentrant patterns. An action potential generated by an electrical stimulus may propagate into a region where the tissue is not fully recovered from the previous excitation and further propagation is obstructed at that point (unidirectional block). The wave is then forced to follow an alternate pathway, which may be provided by an anatomic structure, such as an orifice or scar tissue due to an infarction. Early studies showed that a self-maintained excitatory wave could be created about a ring of tissue; in this case, the obstacle to propagation is the hole in the center of the ring $[17,18]$.

The dimensions of the anatomic obstacle determine the size of the reentrant circuit. If the rotation period (the circuit path length divided by conduction velocity) is longer than the refractory period, there will be an excitable gap, a region between the wave crest and wave tail which consists of excitable tissue. A properly timed stimulus may annihilate or reset the reentry via invasion of the circuit through the excitable gap [19,20].

\subsubsection{Functional Reentry}

Anatomic obstructions are not the only mechanisms of reentry. Functional reentry occurs through conduction block caused by heterogeneities of dynamic electrical properties of normal cardiac tissue, such as the time course of repolarization and recovery of excitability [21]. For example, the leading circle mechanism of reentry [22] occurs when a local disparity in refractoriness causes unidirectional block. Propagation is permitted in areas with shorter refractory periods but halted in regions with longer refractory periods; the wave then circulates back to those areas which were previously refractory but have now recovered excitability. No anatomic obstacle is required, which makes the underlying cause of reentry much more difficult 
to assess. Unlike anatomic reentry, in leading circle reentry there is no excitable gap; the circuit is the minimum permissible length such that the wave crest is sufficient to re-excite the tissue at the relatively refractory wave tail. In addition, the wave front creates centripetal "wavelets" which propagate towards the center. The collision of these wavefronts renders the center completely refractory. The width of the excitable gap is partially determined by the curvature of the turning wavefront $[23,24]$ as well as spatial heterogeneities along the length of the circuit [19].

\subsubsection{Spiral Wave Reentry}

An alternate mechanism is based upon the observation in theoretical studies that reentry may be initiated if the heart is treated as a generic, homogeneous, nonlinear excitable medium. It has been shown that activity analogous to reentry is a ubiquitous feature in such media given the proper conditions and has been observed in a variety of chemical, physical, and biological systems [25-28]. This form of functional reentry manifests itself as a spiral activation pattern with decreasing wave front curvature with increasing distance from the center. The propagation velocity of a cardiac wavefront is strongly dependent upon the curvature of the front. As a result, due to a limit in maximum curvature at the tip of the spiral, the wave does not rotate about the tip itself, but rotates around an organizing center called the core. In contrast to the leading circle form of reentry, the core area is not invaded by wavefronts, but instead is an unexcited but excitable medium that defines the primary dynamic characteristics of the wave [3,29].

Currently, the critical-point hypothesis conceptualized by Wiener and Rosenblueth [30] and later extended by Winfree [31,32] for application to cardiac studies is accepted as the governing principle by which spiral wave reentry is initiated. This theory posits that a spiral wave will form at the intersection between a line of critical refractoriness created by an initial stimulus (S1) and a line of critical field strength imposed by a later, second stimulus (S2); this process is illustrated in Fig. 2.3. In experimental practice and computer simulation, these waves are typically produced by a procedure known as cross-field stimulation, based upon critical-point theory, which is shown in Fig. 2.4A using a simulated model. At 1000 time units (t.u.), an S1 planar wave has propagated across the medium from left to right, creating a spatial gradient of

refractoriness across the tissue. During its progression, a second S2 planar wave is activated via a line stimulus traveling in a perpendicular direction to the first wave, from top to bottom. The 


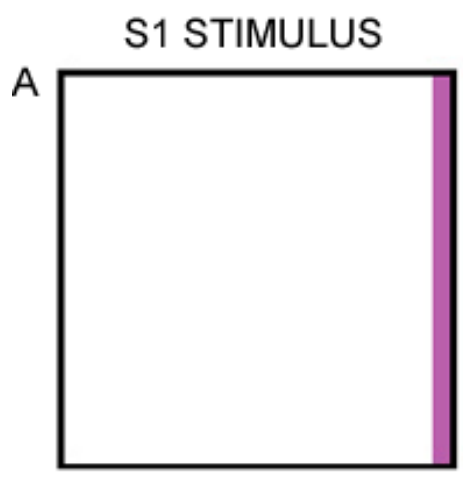

S1 Line

Stimulus

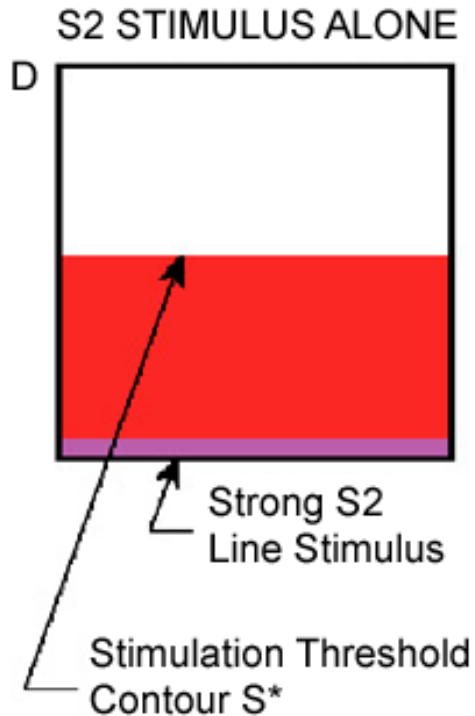

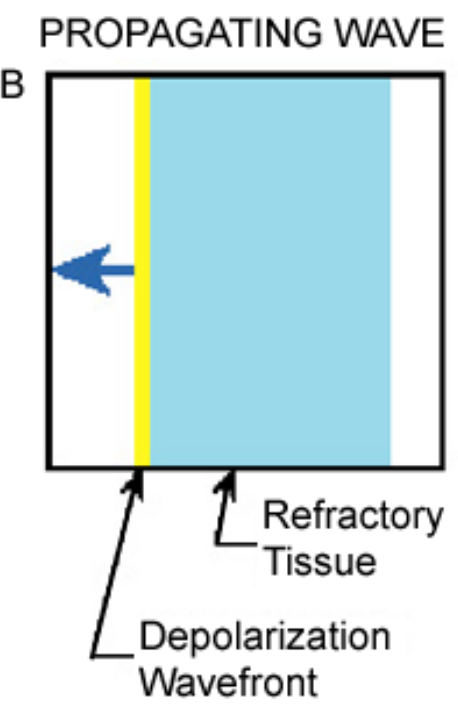
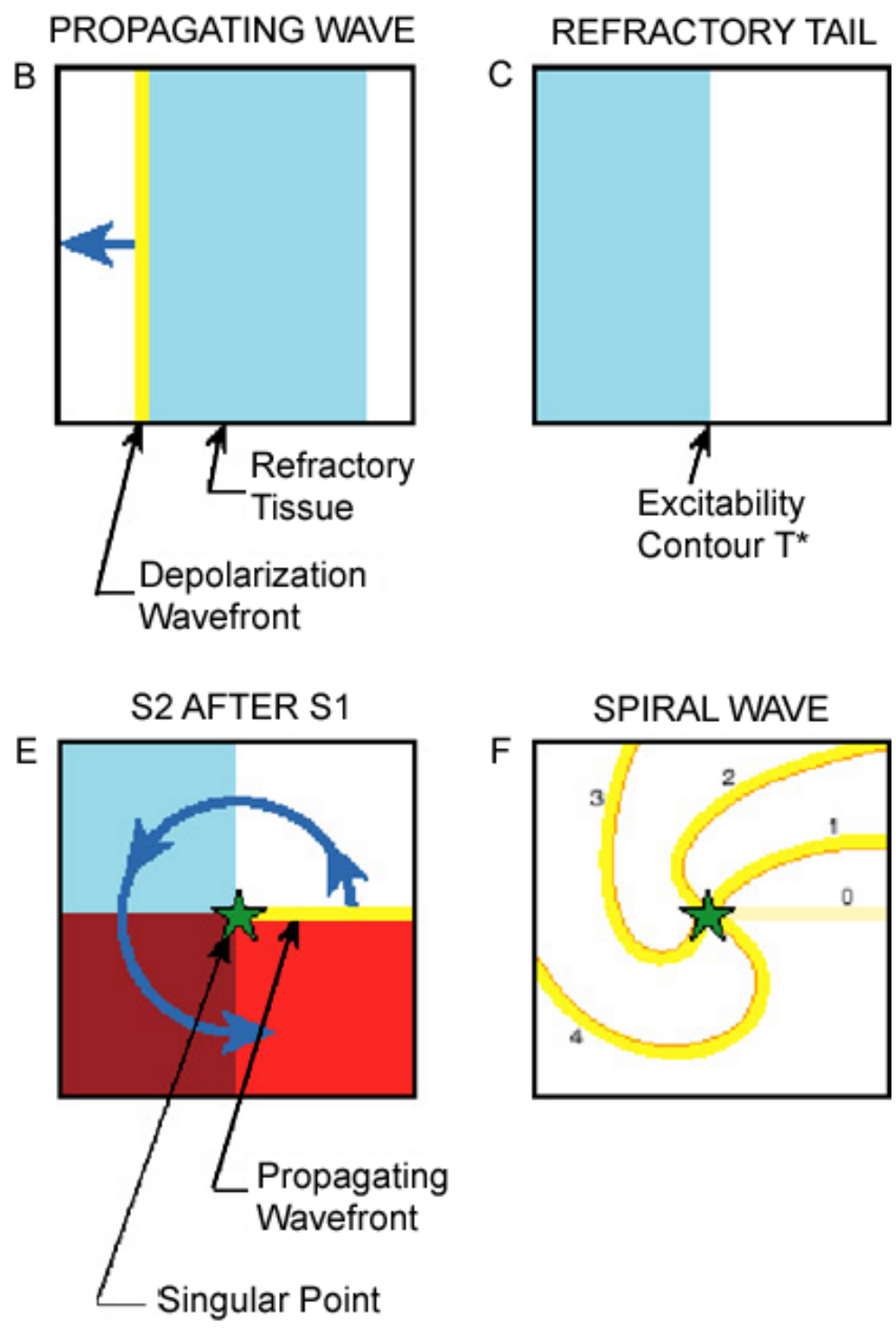

Figure 2.3: Illustration of the critical point hypothesis. A wave is generated by an line S1 stimulus in (A), propagates across the tissue from right to left in (B), creating a critical excitability contour $\mathrm{T}^{*}$ in its refractory wake (blue). At this point, a line S2 stimulus (red) delivered on bottom edge of the tissue produces a critical threshold contour $\mathrm{S}^{*}$ in (D). The intersection of $\mathrm{T}^{*}$ and $\mathrm{S}^{*}$ causes unidirectional conduction block and initiates a spiral wave whose progress is seen in (F). Wavefronts are shown in yellow.

second wave encounters refractory tissue on the right and is unidirectionally blocked. The left side, meanwhile, has recovered excitability, permitting the wave to continue propagation. The wave then curls to the left, following the gradient of refractoriness, creating a counter-clockwise reentrant spiral wave. In Fig. 2.4A, at 2300 t.u., the spiral wave has undergone one full rotation. Similar conditions with an initial planar wave followed by a properly timed point stimulus create 


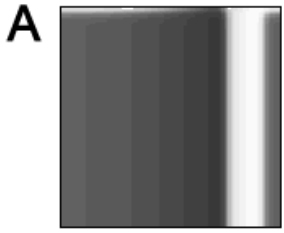

$\mathrm{t}=1000 t . u$.

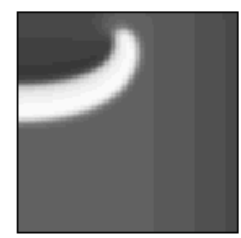

1500

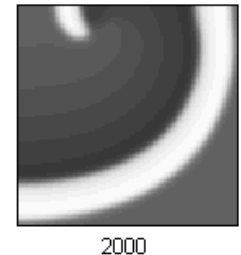

B

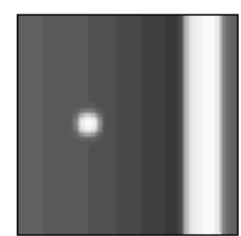

$t=1000$ t.u.

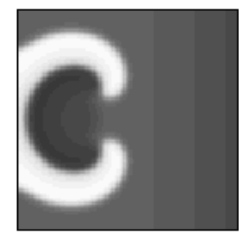

1500

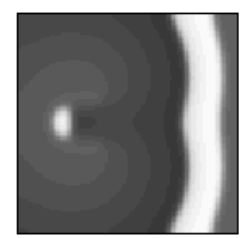

2000

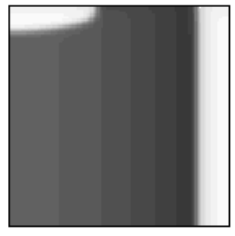

1100

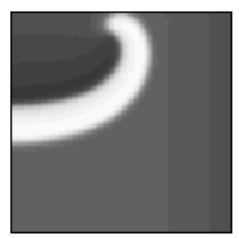

1600
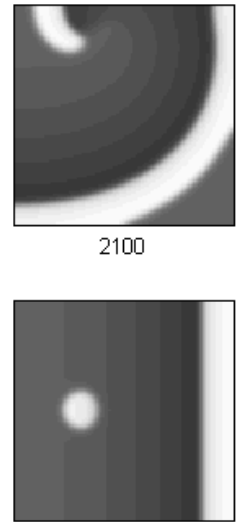

1100

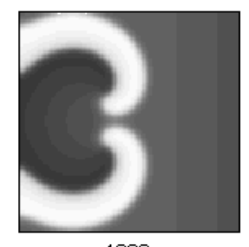

1600

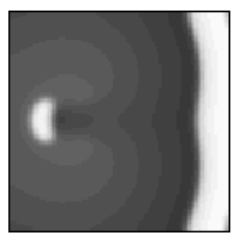

2100

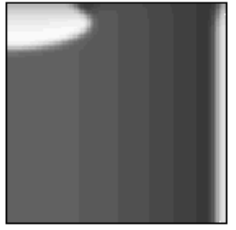

1200

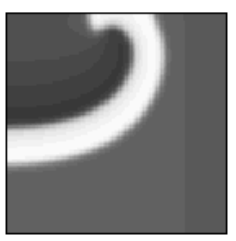

1700
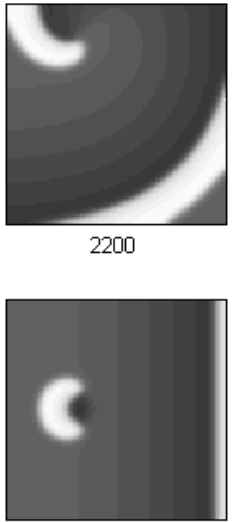

1200
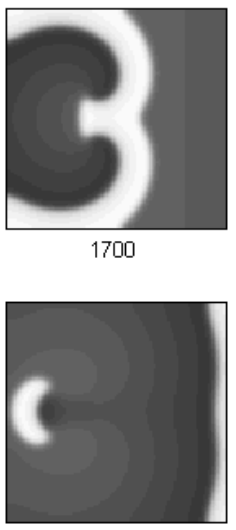

2200

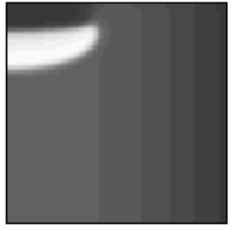

1300

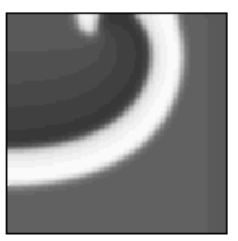

1800
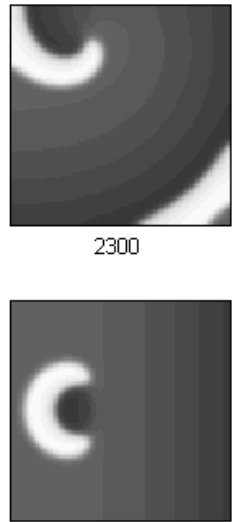

1300
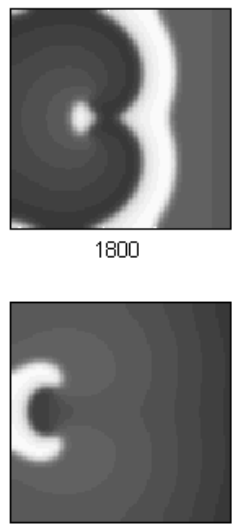

2300

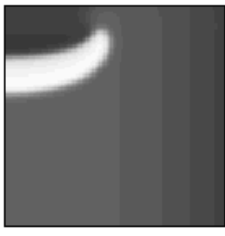

1400

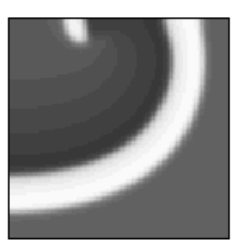

1900
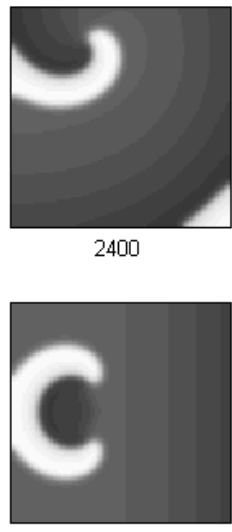

1400
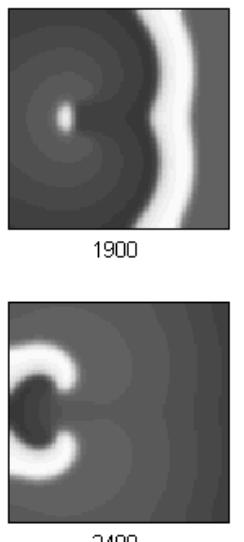

2400

Figure 2.4: Creation of (A) single spiral and (B) figure-of-eight reentry via cross-field stimulation in a FitzHugh-Nagumo model. White denotes depolarized tissue, black denotes hyperpolarized tissue, gray denotes resting tissue. Labels underneath indicate time step in simulation. S2 is applied at 1000 t.u. 

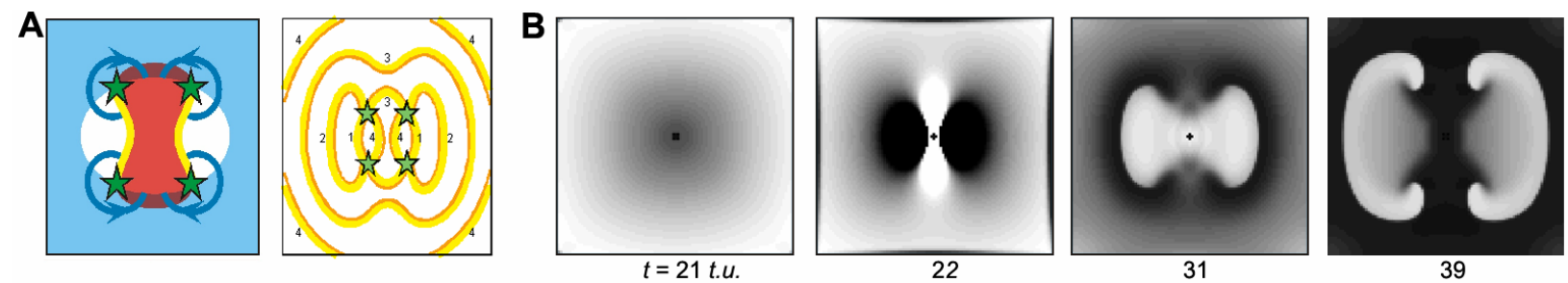

Figure 2.5: (A) Illustration of the critical point hypothesis in quatrefoil reentry for cathode break S2 stimulation. In the left panel, the refractory wake created by the S1 stimulus is shown in blue, the depolarization at the virtual cathode created by the S2 stimulus is shown in red, and the hyperpolarization at the virtual anode created by S2 stimulus is shown in white. The right panel shows the progression of the four spiral waves. Wavefronts are shown in yellow. (B) Creation of quatrefoil reentry in a Beeler-Reuter model. White/gray: depolarized tissue, black:

resting/hyperpolarized tissue. At $t=21 t . u$, the tissue is still refractory from the S1 stimulus just prior to the $\mathrm{S} 2$ stimulus at $t=22 t . u$.

a pair of counter-rotating spiral waves, a spiral pattern known as "figure-of-eight" reentry [32], as shown by the simulation in Fig. 2.4B. Both of the single spiral wave and figure-of-eight reentries have received extensive attention in the literature [23,33-38].

Another interesting form of reentry is that initiated by S1-S2 stimulation through an electrode at one location (unipolar), as opposed to two separate locations as described previously. The formation of this reentrant circuit is dependent on a peculiarity of the bidomain membrane model described in Section 2.2.3, the presence of virtual electrodes. When a cathodal electrode stimulates cardiac tissue, it gives rise to a "dog-bone"-shaped depolarization pattern around the electrode, oriented perpendicular to the longitudinal fiber axis; this polarization distribution is called a virtual cathode. Counter-intuitively, regions of hyperpolarization flanking the cathode also appear parallel to the fiber axis; these are termed virtual anodes. The presence of virtual electrodes has been shown to be a direct consequence of the fact that cardiac tissue possesses unequal anisotropy ratios in the extracellular and intracellular spaces [39]. The virtual anode has the effect of shortening the refractory period of the S1 wavefront, while the virtual cathode lengthens the refractory period. Therefore, at the termination of the unipolar S2 stimulus ("break" stimulation), the resulting wavefront is unable to propagate into the depolarized virtual cathode region but is able to propagate into the hyperpolarized virtual anode region due to unidirectional block. The progression of events is depicted in Fig. 2.5. This reentrant pattern is termed "quatrefoil" reentry since the juxtaposition of the depolarized and hyperpolarized areas 
forms four critical points, and hence four spiral waves. Numerical predictions of this form of reentry have been borne out experimentally $[40,41]$.

\subsubsection{Spiral Wave Motion}

Spiral waves may remain stationary or may drift, giving rise to multiple, dynamic reentrant pathways [37,42]. Much of the computational work into spiral wave motion has made use of the FitzHugh-Nagumo (FHN) model [5], a simple, two variable system which is a generalization of the basic properties of an excitable medium. [43]Variation of the parameters in the model demonstrated a variety of cycloidal and elliptical patterns of meander and it has been shown that the threshold for excitation and the ratio of the recovery rate to excitation rate are the dominant parameters in determining spiral wave trajectory [43-45]. Later work utilizing higher order, more complex models exhibited the same essential patterns [46,47].

The presence of a parameter gradient has been shown to cause spiral wave drift which has been examined both analytically [48] and experimentally in the Belousov-Zhabotinsky (BZ) oscillating chemical reaction [49,50] as well as cardiac tissue [51]. Furthermore, the imposition of a gradient via periodic forcing of a system parameter at the resonant frequency of the spiral permits a degree of control over the trajectory of the wave [52,53]. The possibility of eliminating spiral waves by externally driving them into regions of tissue where they cannot be sustained has obvious applications to the field of defibrillation and is currently under investigation [46,54].

The interaction of rotors has been a topic of extensive discussion in the literature, both numerically and theoretically [55-59]. In the complex Ginzburg-Landau equation (CGLE) system, analytical solutions have been found for various parameter ranges and indicate that the interaction between spiral waves is fairly weak, decaying exponentially over short separation distances [60] (for a comprehensive review of CGLE vortex interaction, see [59]). However, a corresponding analytical solution for the FHN system interaction dynamics has yet to be found. Because the correspondence between the parameter values in the CGLE and those in a physical system is not clear, it may not be possible to replicate these theoretical behaviors in an experimental preparation. Still, the case for the existence of bound states between spirals has been advanced theoretically for the CGLE and numerically for the FHN models [56,61]. In particular, a bound state between spirals of the same chirality would correspond to a multi-armed spiral pattern. Such behavior has in fact been studied in some experimental settings such as the 
$\mathrm{BZ}$ reaction, among others $[62,63]$, although theoretical evidence suggests that they are unstable under conditions of weak excitability or as the number of arms increases [64,65]. However, no evidence exists that these two phenomena occur in cardiac tissue, other than in cases where local structural heterogeneities cause spiral wave pinning [66].

A significant amount of research is centered on the finding that excitation inhomogeneities within the medium will cause frequency-dependent spiral wave interaction. That is, a spiral wave with a higher frequency of rotation will tend to repel its slower-rotating neighbors. This phenomenon has been observed experimentally for the BZ reaction $[67,68]$ and simulated numerically on a FHN-type model [69], the Ginzburg-Landau system [70,71], as well as more physiologically realistic cardiac models [72].

Much of the literature describing observations of the motion of spiral waves of cardiac tissue is qualitative; for example, it is well known that a pair of cardiac spiral waves of opposite chirality and sufficient proximity will attract one another and annihilate due to conduction block between the pair. However, substantive quantitative studies of spiral interactions over time in experimental preparations are almost non-existent ([73] is an exception). Much of the reason for this paucity is the fact that often the observed spirals are involved in full-blown fibrillation, at which point the behavior is already so complex that the relationships between nearby spirals are difficult to determine. The possibility of using a controlled, repeatable means of generating multiple spiral waves to overcome this issue will be explored later in this dissertation.

\subsubsection{Three-dimensional spiral waves}

Scroll waves are the three-dimensional analogue of spiral waves, and may be visualized as a stack of spiral waves extending through the bulk myocardium. The three-dimensional line connecting the two-dimensional core regions in the stack is termed a filament, around which the scroll wave rotates. Hence, a spiral wave observed on the epicardial or endocardial surface simply becomes the cross-section of the scroll wave where it has come into contact with a boundary.

Observation of the BZ reaction provided the first demonstration of scroll waves [74]. The three-dimensional shape of the filament need not remain fixed; filament morphology can assume one of any number of forms, leading to the evolution of extremely complex scroll wave dynamics [75]. For example, a filament may extend transmurally (across the myocardial wall) to 
form an I-shape, presenting as a single spiral wave on the surface. A U-shaped filament occurs when the filament ends terminate on the same surface; the surface manifestation would appear as figure-of-eight reentry, such that the two counter-rotating spiral waves are actually endpoints of the same scroll wave. A filament forming a closed loop within the bulk myocardium is commonly known as a scroll ring, with no reentrant activity discernable on the surface but instead exhibiting the periodic appearance of "breakthrough" activations as the outer portion of the scroll wave reaches the epicardium. Thus, categorization of the scroll wave pattern on the basis of surface electrophysiological recordings is extremely difficult due to the variability of filament configuration.

\subsubsection{Scroll waves and ventricular arrhythmias}

A persistent question is exactly how isolated spiral waves spontaneously break up to multiple complex reentrant pathways, and thus, what was once ventricular tachycardia (VT) degenerates into VF. Moreover, it has been shown that this instability appears not necessarily as a result of the presence of homogeneities in the cardiac properties as once thought, but also as a consequence of the inherent dynamics of an excitable medium [3].

While two-dimensional numerical simulations can generate VF-type behavior from spiral wave reentry (see [76] for a review of mechanisms by which this may occur) the threedimensional aspect of the myocardial wall, in light of experiments, cannot be ignored. Hence, another debate involves the question of whether scroll wave breakup is responsible for VF induction. Despite the difficulty of tracking scroll waves in experiments, evidence suggests that polymorphic VT corresponds to the presence of a single, drifting scroll wave oriented perpendicular to the surface [37]. While additional experimental evidence is not yet forthcoming, the hypothesis that VF is a direct result of filament instability leading to filament proliferation is generally accepted, and there is a rapidly growing body of literature involving numerical studies investigating the parameters under which scroll waves destabilize and break

One mechanism which is considered to be important in filament destabilization is the presence of rotational anisotropy in cardiac tissue. The muscle fibers lie in sheets tangent to the epicardial and endocardial surfaces, but longitudinal axis of each layer is not parallel between sheets. Rather, they undergo a roughly $120^{\circ}$ rotation from epicardium to endocardium, an amount which has been found to be fairly consistent between species [77]. This angular fiber 
rotation has implications on filament orientation [78,79] but in particular, it has a important effect on the rate of angular rotation of the scroll wave around the filament as a function of arclength, a quantity known as twist. It has been shown numerically that a buildup of twist along the length of the filament significantly decreases stability, and that this twist is largely generated by the rotational anisotropy of the tissue [80-82]. This indicates that not only the intrinsic properties of the excitable medium but also the structural heterogeneity of normal cardiac tissue may facilitate the formation of VF.

Studies indicate that the thickness of the myocardial wall is an important factor in the initiation of VF; the threshold value of wall thickness has been estimated as $1 / \pi \times$ (distance the wave travels during one rotation), giving a minimum value of roughly $3 \mathrm{~mm}$ [83]. Other numerical studies have since demonstrated that tissue thickness is a factor in determining filament stability [82,84-86]. To validate this hypothesis, in vitro studies can create a thin layer of myocardium by freezing the endocardium and much of the intramural layers, leaving the epicardium intact. Such experiments confirm that a minimum wall thickness, about $3-4 \mathrm{~mm}$, is needed to generate VF, otherwise sustained VT occurs [87,88]. Human left ventricular myocardium is on the order of $1 \mathrm{~cm}$, and hence is thick enough to support filament breakup.

Filament tension, a parameter which describes the whether the filament length increases or decreases with time, has been implicated as a cause of instability of scroll waves embedded in weakly excitable tissue [89]. If the tension is positive, perturbations to a linear filament are damped (a filament with kinks will straighten) or a scroll ring will eventually collapse as it acts to minimize its length. However, if the tension is negative, perturbations to an initially straight filament grow with time, causing the filament to lengthen. The filament will eventually either break upon the bounding surfaces in a bounded medium or pinch off within the interior of the medium and generate additional filaments. While ordinarily cardiac tissue is highly excitable, the presence of ischemia (as a result of coronary artery occlusion, for example) decreases excitability and may produce regions of negative tension. 


\subsection{Phase portrait analysis}

\subsubsection{Introduction}

The utility of analyzing dynamical systems in terms of geometric trajectories within a phase portrait has been established for over a century and applied to a variety to physical problems. The power of this approach lies in the ability to examine the behavior of the system in question without the need to solve it explicitly, in that each point illustrates the behavior of the

full system at a given point in time. In particular, the dynamics of excitable media are usually composed of coupled first-order autonomous differential equations, and hence naturally lend themselves to analysis in the phase plane.

\subsubsection{Applications to cardiac models}

Differential equations describing the ionic basis of nerve action potentials were derived by Hodgkin and Huxley [4], followed by analytical studies by FitzHugh which illustrated the oscillatory behavior of the equations by the use of phase space and explored qualitative properties by phase plane analysis [5]. The FitzHugh-Nagumo (FHN) equations, derived and simplified from the Hodgkin-Huxley model and closely related to the van der Pol oscillating system, are now commonly used as a generic, two-variable model of excitability with the following formulation:

$$
\begin{gathered}
\dot{u}=D_{u} \nabla^{2} u+c\left(v+u-u^{3} / 3\right), \\
\dot{v}=D_{u} \nabla^{2} v-(b u-a+v) / c,
\end{gathered}
$$

with $1-2 b / 3<a<1,0<b<1$, and $b<c^{2}$. The variable $u$ corresponds to the membrane potential, which changes rapidly and has a large diffusion coefficient, $D_{u}$. The variable $v$ corresponds to a measure of refractoriness, which changes slowly and has a small diffusion coefficient, $D_{v}$; for cardiac models, $D_{v}$ is set to zero. Other similar two-variable formats use the same basic theme; see [43] for a review and comparison of various formulations.

The phase for a sample FHN system without diffusive coupling is shown in Figure 2.6 (i.e., a 0 -D point). A single stable fixed point is seen at the intersection of the nullclines, corresponding to the system in a resting state. A suprathreshold change in $u$ will cause the state 

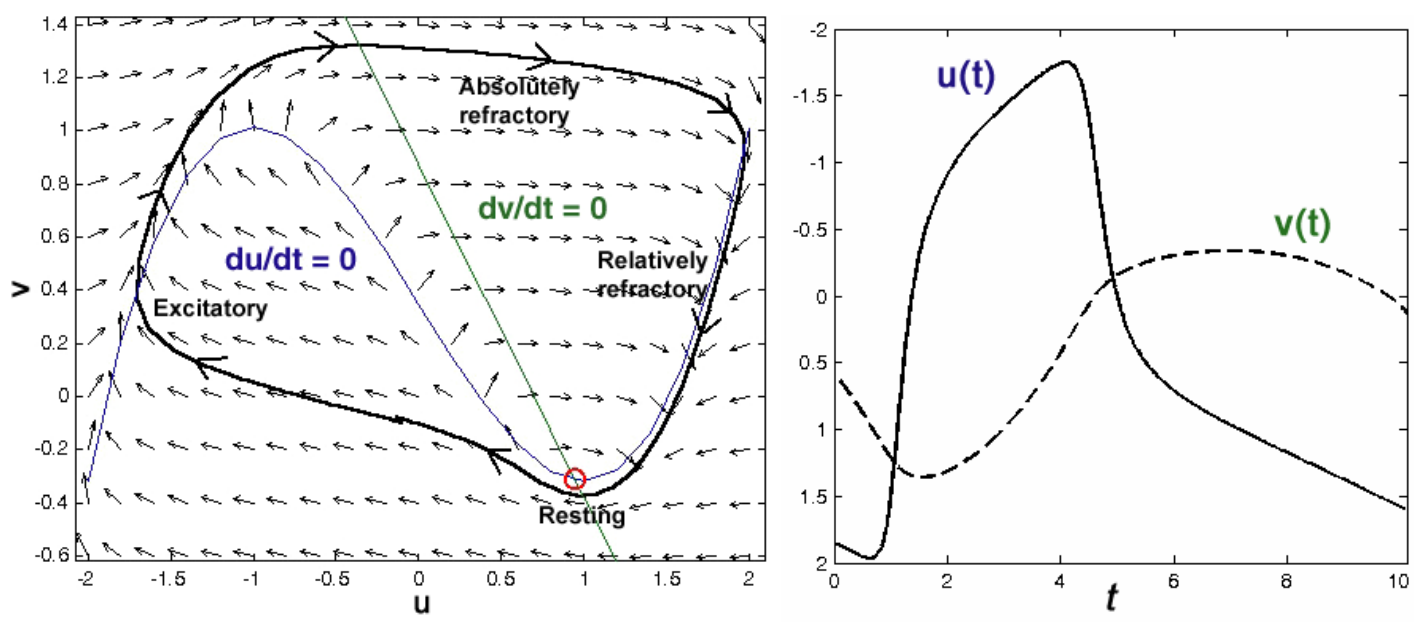

Figure 2.6: Phase portrait for FHN equations ( $a=0.7, b=0.8, c=3)$ showing electrophysiological states of $(u, v)$ on the left, time traces of $u$ and $v$ on the right. A stable fixed point, shown as a red circle, occurs at the intersection of the two nullclines (green and blue lines). Arrows show the direction field. A sample trajectory is shown as a thick black line.

variables to undergo a rapid excursion towards the left (corresponding to depolarization). The trajectory progresses slowly up the left branch of the $u$ cubic nullcline until it reaches the local maximum, at which point it moves rapidly right. It then travels slowly down the right $u$ nullcline branch and returns to the rest state. In this way, it reproduces the significant qualities of an excitable system, namely a closed loop trajectory under the conditions of a sufficiently large perturbation, even though a stable fixed point exists. Even so, the FHN system remains a caricature of a cardiac action potential; it fails to reproduce the specific characteristics unique to cardiac ionic kinetics, such as upstroke and repolarization times as well as restitution properties (the relationship between the action potential duration and the duration of the previous rest period). Several attempts have been made to maintain the two-variable simplicity of the FHN formulation while being more physiologically realistic [90,91].

For practical purposes, an experimenter may not have direct access to multiple, concurrent state variables in order to perform a dynamical systems analysis. However, a topologically equivalent attractor may be reconstructed given only one component of the system $[92,93]$, a procedure known as times-series analysis. For $N$ evenly sampled values of $y(t)$, the attractor in two dimensions is

$$
y i=[y(i), y(i+\tau)],
$$

where $\tau$ is the time-embedding lag and $i=1, \ldots, N-1$. 
A

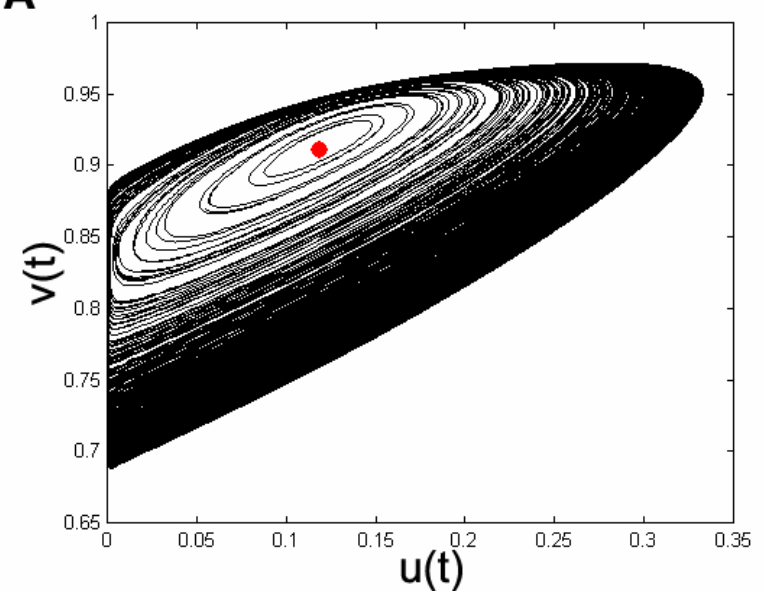

B

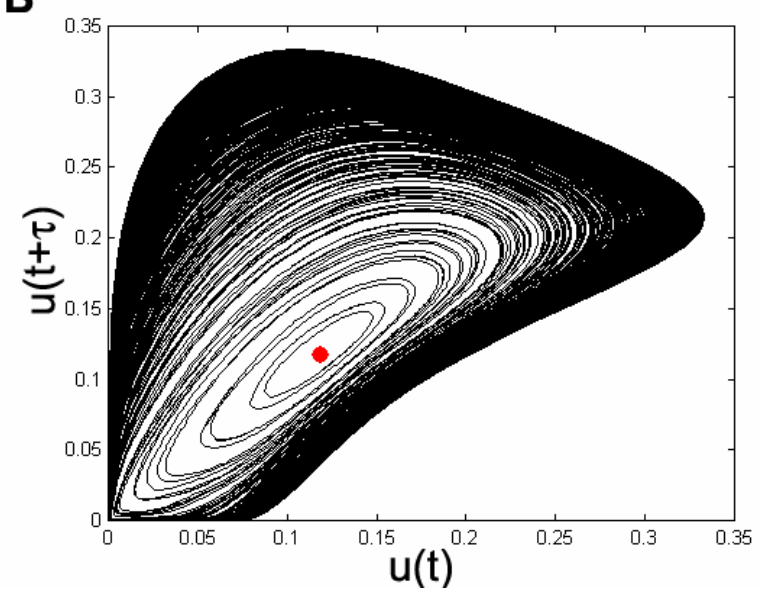

Figure 2.7: Examples of phase portraits for the BZ two-variable $(u, v)$ model. (A) $u(t)$ against $v(t)$. (B) $u(t)$ against $u(t+\tau)$. Red circle indicates origin.

In experimental studies, typically the single observable variable is the transmembrane potential in cardiac tissue, $V(\vec{r}, t)$ (we drop the usual subscript for this section) at a given spatial location $\vec{r}$. To reconstruct the attractor in phase space, we create a second variable, $V^{\prime}(\vec{r}, t)$, which we define by time-delay embedding of $V$,

$$
V^{\prime}(\vec{r}, t) \equiv V(\vec{r}, t+\tau) .
$$

In the dynamical literature, the question of how to select an optimal $\tau$ for a particular system has been studied extensively [94]. Generally speaking, it is desirable to choose $\tau$ such that the correlation between $V$ and $V^{\prime}$ is minimized; for example, Gray et al. [73] calculated $\tau$ as the first zero-crossing of the autocorrelation function of $V(\vec{r}, t)$. However, the autocorrelation function insures only linear independence between signals, so typically calculation of the average mutual information is the preferred method of choosing $\tau$ since it is applicable to nonlinear signals as well [95]. It is also worth noting that newer studies are investigating the feasibility of obtaining simultaneous optical recordings of the transmembrane potential plus an additional parameter (such as calcium) in order to circumvent this issue [96,97], despite the technical difficulties of using a multi-imager system and minimizing of cross-talk between the spectra of multiple fluorescent dyes $[98,99]$.

Once the additional variable has been generated, we can represent the temporal behavior of an excitable element in phase space as a closed path, termed a limit cycle. We can then define 
the local phase of this element, $\phi(\vec{r}, t)$, in terms of the angle around the limit cycle in phase plane, referenced to an origin. One formulation is the following:

$$
\phi(\vec{r}, t)=\arctan \frac{V(\vec{r}, t+\tau)-V_{\text {mean }}(\vec{r})}{V(\vec{r}, t)-V_{\text {mean }}(\vec{r})},
$$

where $V_{\text {mean }}(\vec{r})$ is the mean value of $V(\vec{r}, t)$ [73]. An example of a phase plane reconstruction is shown in Fig 2.7 for the BZ two-variable model. However, other schemes to express phase may be defined, and there exist additional concerns which make the reconstruction of the attractor derived from a cardiac signal a special case, particularly in the choice of $\tau$, both of these topics will be explored later in this dissertation.

\subsection{Phase singularities and filaments as topological defects}

As a result of the observation of vortex-like behavior commonly observed in nonlinear systems, the terminology of nonlinear dynamics is beginning to appear in the cardiac electrophysiology literature. One such concept in particular is that of the topological defect, which is a discontinuity in an ordered parameter field that cannot be removed by continuous deformation of the local field [100]. Furthermore, if the defect is enclosed by a continuous closed loop, the value of the order parameter as the loop is traversed changes as a multiple of $2 \pi n$, where $n$ is an integer known as the winding number. While the concept of the topological defect, well known in disciplines such as crystallography, was not applied to cardiology until fairly recently, providing an interpretation of the resetting of phase in a functional reentrant circuit [32]. An example of phase resetting may be illustrated by the mechanism of cross-field stimulation described in Section 2.3.4. If the stimulus is suprathreshold in strength, it has the effect of shifting, or resetting, the phase of the membrane into a new cycle (even-resetting); if the stimulus is subthreshold, the phase is reset but remains within the current cycle (odd-resetting). Provided the timing is correct, the stimulus creates neighboring regions upon the membrane which will experience even- and odd-resetting. The adjacent spatial discrepancy in phase will generate a point about which the phase will span all possible values in an orderly manner. Using the terminology defined above, we are defining phase $\phi$ as the order parameter in this situation; a closed loop about the defect will progress through a full $2 \pi$ of phase, hence a winding number of 
\pm 1 . In this context, a phase singularity is defined as the point in space where the gradient of the phase diverges, i.e., $|\nabla \phi|$ becomes infinite and the phase itself at this point is undefined.

On the basis of the above mathematical abstraction, theoretical topological analysis of the phase distribution during vortex-like activity indicates that a phase singularity must exist within the core of a spiral wave $[73,101]$. Furthermore, as a 2-D point defect is a cross-section of a 3-D line defect, by analogy, a spiral wave phase singularity can be seen to be the surface manifestation of a scroll wave filament. It should be noted that while the defect is a discontinuity in the order parameter, it does not correspond to a discontinuity in any actual physical quantity. Nonetheless, this description yields useful information on the characteristics of scroll waves. Topological analysis shows that the filament can never terminate in the myocardial bulk but instead the ends are restricted to either external boundaries or pinned to a closed bounding surface [102]. Similar arguments can be made describing the nature and constraints of filament reconnection [103,104]. Numerical simulations have indicated that filaments can be created in exotic configurations, such as intricate knotted rings [105,106]; in addition, simple scroll rings and twisted filaments have been observed in the BZ reaction [107-110]. However, how many of these patterns are realizable in the myocardium, as well as their stability once formed, have yet be discovered. At this point, we can see how the discussion from the previous section (Eq, (2.9) in particular) is applicable in creating the phase order parameter from a set of data, whether is experimentally obtained or numerically simulated.

Further topological constraints provide additional properties of the phase singularity. For example, a suitably large contour around a pair of topological defects yields a winding number equal to the sum of the individual winding numbers. Effectively, a pair of defects can be transformed into a single defect with the total net winding number provided they are brought into proximity with each other, such that the contour around them approaches an infinitesimal length. Therefore, removal of a topological defect requires that a second defect with the opposite winding number be brought into contact to produce a net zero winding number; driving a single singularity into a boundary produces the same result [100]. At short distances, it has been experimentally established that vortices with opposite direction of rotation can annihilate [73], in accordance with mathematical theory; the question of whether nearby vortices attract or repel (or even form stable bound paired states) is determined by the parameters of the simulated excitable system and is poorly understood for cardiac tissue. 
The CGLE system (mentioned in Section 2.3.6) given by

$$
\frac{\partial A}{\partial t}=A-(1+i \alpha)|A|^{2} A+(1+i \beta) \nabla^{2} A
$$

has proven amenable to investigation of filament dynamics in part due to the fact that an explicit solution can be obtained for a single-armed spiral,

$$
A_{0}(r, \phi, z, t)=F(r) \exp \left\{i\left[-\omega_{0} t+\sigma \phi+\psi(r)+k_{z} z\right]\right\},
$$

where $(r, \theta)$ are polar coordinates, $\omega=-c-k_{0}^{2}(1-\varepsilon c)$ is the rotation frequency, $k_{z}$ is the axial wavenumber, and the functions $F(r)$ and $\psi(r)$ have the asymptotic behavior such that as $r \rightarrow \infty$,

$F(r) \rightarrow \sqrt{1-\varepsilon k_{0}^{2}}, \psi^{\prime}(r) \rightarrow k_{0}$, and as $r \rightarrow 0, F(r) \sim r, \psi^{\prime}(r) \sim r$. The important item to note here is not only does an explicit solution to (2.10) exist, but also that in (2.11), the parameters $\sigma$ denotes the topological charge, equal to \pm 1 in this case, and $\phi$ is the phase field; both of the important order field parameters are "built-in," so to speak, into the solution $A_{0}$. For this reason, the CGLE system has been examined extensively for stability [111], reconnection [103], twist [112] and motion [113] of singularities and filaments.

\subsection{Cardiac optical mapping}

\subsubsection{Introduction}

Traditionally, measurements of cardiac electrical activity have been obtained via extracellular electrograms or glass microelectrodes. However, optical fluorescence imaging has emerged as an alternate technique to perform cardiac mapping since its development in 1974 and its first successful application to cardiac tissue in 1976 [114,115]. This methodology involves monitoring changes in $V_{m}$ with the use of dyes whose fluorescence is potentiometric (i.e., voltage-dependent). The dye molecule lodges in the cell membrane where, in response to a change in the local transmembrane potential, undergoes a charge shift, changing the optical properties of the dye and causing a shift in the fluorescence peak such that that fluoresced signal has an intensity proportional to $V_{m}$. In practice, $V_{m}$ at a recording site is estimated as $-\Delta F / F$, where $F$ is the fluorescence intensity obtained from electrically resting tissue at a particular location in the heart, and $\Delta F$ is the difference between $F$ and the intensity when the tissue is excited. A depolarization produces a fluorescence shift towards shorter wavelengths, which is manifested as a downward deflection in the recorded long-wavelength signal. The minus sign is 
added to invert the signal so that action potentials are displayed with the standard orientation recorded electrically. Excitation may be spatially localized using a laser beam [116,117] or over large areas of the heart using optical fibers or diffusers [24,118].

\subsubsection{Advantages of optical mapping}

Optical dyes have been used in studies that explore the mechanisms in which a defibrillation shock halts fibrillation [119], discern the spatiotemporal patterns of fibrillation [73], and verify theoretical computational cardiac models of propagation [13]. Optical cardiac mapping offers several advantages over traditional glass microelectrode or extracellular recordings

1. It is a non-invasive technique: The use of glass microelectrodes requires the insertion of a probe in the intracellular space of the myocyte through the cell membrane. In some circumstances, (such as studies of embryonic heart cells), cell impalement may be undesirable since it damages the cell [120]. Insertion of the probe may be extremely troublesome because of the small size of the cell (the length of a cardiac myocyte is typically $100 \mu \mathrm{m}$, with a width of 30-50 $\mu \mathrm{m}$ ), or it may be unstable because the tissue is moving due to mechanical contraction. Maintaining stable electrophysiological properties may also be difficult. The use of dye that is localized to the lipid bilayer circumvents these problems

2. Optical mapping permits examination of a large surface area with high resolution: The spatial observation of the specific extent of cardiac arrhythmias is necessarily a multi-scale and multi-site measurement. Reentrant excitation may originate over an area as small as $2-4 \mathrm{~mm}^{2}$ [121], requiring high-resolution mapping, but then give rise to spiral waves which may circulate over the entire ventricular area [37], which necessitates observation at many locations. Multi-site recordings are possible with contact electrodes arrays, but electrode size and density, and array complexity place an upper limit on the number of data channels allowed. Furthermore, stable recordings are dependent upon uniform electrode contact, which is difficult to achieve with a large electrode array. Optical recording allows for simultaneous recording from hundreds or thousands of cardiac sites, with a spatial resolution that increases linearly with magnification with a limit on the order of 50-100 $\mu \mathrm{m}$ [122].

3. It can directly observe both depolarization and repolarization: The primary advantage of extracellular recording over microelectrodes is the fact that the activity of cells may be 
recorded without impalement and resultant damage. However, extracellular recording is limited to sampling the extracellular potential field without measuring the underlying source, the cardiac action potential. Examination of the action potential upstroke during depolarization is essential for determination of conduction velocity and cycle length, both of which are altered in reentrant pathways [36,123]. Studies indicate that the prolongation of repolarization for fibrillating waveforms may be critical to the process of defibrillation [124]. Since optical mapping is a measurement of $V_{m}$, which reflects membrane behavior, and is less dependent on the geometry of the cell and the electrode, the information obtained is better suited to examining local cardiac depolarization and repolarization.

4. The optical technique is not adversely affected by stimulus artifacts during an electrical pulse: The recording of surface potentials are usually complicated by the presence of a stimulus artifact produced by the extracellular electrical field. The artifact is usually manifest as a spike followed by a time decay, the amplitude and time constant of which is dependent upon on the characteristics of the stimulator, electrodes, and the filtering effects of the preamplification stage [125]. The superposition of the artifact upon the physiological signal precludes data capture for several milliseconds after a stimulus, unless differential extracellular recordings or special amplifier systems are used [126]. The presence of a stimulus artifact is an accepted part of microelectrode recording, which can be diminished but usually not removed entirely. In the case of optical recording, in which $V_{m}$, rather than the extracellular field, is the parameter examined, the contribution to the signal from the stimulus is much smaller $[119,127]$. This attribute can provide crucial information about electrical behavior associated with strong stimuli, such as virtual electrode effects [128]

5. Changes in fluorescence are rapid: Experiments using the dye RH 237 demonstrated that the dye is capable of reproducing action potential upstroke rates faster than equivalent microelectrode measurements, on the order of a few microseconds [129]. Other dyes are capable of response times faster than the swiftest changes in transmembrane potential [130].

\subsection{Summary and thesis outline}

This chapter provided a general overview of the behavior of the heart, both in the normal healthy state and the pathological arrhythmic state. The reentrant circuit was presented as the basic unit of arrhythmic behavior and the current conceptual models and behavior of reentrant 
excitation with corroborating theoretical and experimental evidence were illustrated. The role of the three-dimensional structure of the cardiac tissue and its implications for the observation of reentry has been examined. Also, phase portraits as a means of nonlinear analysis was described as a potential starting point for the study of cardiac dynamical systems. Lastly, the section concluded with an explanation of optical mapping with the use of voltage-sensitive fluorescent dyes as a viable non-contact means of obtaining cardiac data with high spatial and temporal resolution.

The purpose of this thesis is to explore the dynamics of spiral and scroll wave formation and interaction and the electrical activity evident at the epicardial surface as well as within the underlying myocardial substrate. To this end, we will apply novel techniques of data visualization and phase portrait analysis. The thesis is organized as follows:

Chapter 3 will detail the development of an algorithm which detects phase singularities in an efficient and robust manner using topological charge. This portion also includes an initial comparison of singularity behavior during quatrefoil reentry obtained via experimental protocol and numerical simulation. This chapter acts the primary impetus for much the remaining work.

Chapter 4 continues development of the algorithm derived in Chapter 3 to overcome some of the limitations of using the time-delay embedding method. Specifically, we refine the variables used to create the phase portrait and further define the origin point around which the phase is calculated.

Chapter 5 further extends the algorithm in Chapter 3 from 2-D singularities detection to 3-D filament localization. Also, a quantitative comparison of topological defect detection using time-delay embedding and two-variable phase portrait reconstruction will be presented, as well as providing criteria in determining which two variables from a multivariate ionic kinetic model are optimal for phase portrait reconstruction.

Chapter 6 attempts to address the discrepancy in experimental and theoretical singularity interaction results from Chapter 3. Here, we explore a simplified filament system of quatrefoil reentry corresponding to that expected experimentally.

Chapter 7 incorporates more physiologically realistic factors into filament observation, in particular optical depth effects from using voltage-sensitive dyes. This section will discuss implications for future experimental observations of epicardial activation activity. 
Appendix A1 provided a demonstration that singularity detection on a whole-heart basis is indeed possible. An imaging system was developed previously for observation of the entire epicardial surface and was employed for visualization of small-scale cardiac electrodynamics. This section was regulated to an appendix due to the brevity of the manuscript.

Chapter 8 will summarize the results from Chapters 3 through 7 and Appendix A1 and will discuss the overall themes of this thesis, as well as giving limitations and presenting future directions of the presented work. Also, the research considerations and the societal implications are discussed.

\subsection{References}

[1] Myerburg, R. J., Kessler, K. M., and Castellanos, A., "Sudden cardiac death: Structure, function and time-dependence of risk." Circulation, vol. 85, no. 1 Suppl, pp. I2-I10, 1992.

[2] Prevost, J. L. and Batelli, F., "Sur quelques effets des descharges electriques sur le coeur des mammiferes." Comptes RendusSeances Acad Sci, vol. 129 pp. 1267-1268, 1899.

[3] Zykov, V. S., Simulation of Wave Processes in Excitable Media. Manchester: Manchester University Press, 1987.

[4] Hodgkin, A. L. and Huxley, A. F., "A quantitative description of membrane current and its application to conduction and excitation in nerve." J Physiol (Lond), vol. 117 pp. 500$544,1952$.

[5] FitzHugh, R., "Impulses and physiological states in theoretical models of nerve membrane." Biophys J, vol. 1, no. 6, pp. 445-466, 1961.

[6] Beeler, G. W. and Reuter, H., "Reconstruction of the action potential of ventricular myocardial fibres." J Physiol (Lond), vol. 268, no. 1, pp. 177-210, 1977.

[7] DiFrancesco, D. and Noble, D., "A model of cardiac electrical activity incorporating ionic pumps and concentration changes." Phil Trans R Soc Lond B, vol. 307, no. 1133, pp. 353-398, 1985.

[8] Luo, C. H. and Rudy, Y., "A dynamic model of the cardiac ventricular action potential. I. Simulations of ionic currents and concentration changes." Circ Res, vol. 74, no. 6, pp. 1071-1096, 1994.

[9] Rall, W., "Core conductor theory and cable properties of neurons.," in Kandel, E. R. (ed.) The Nervous System: Cell Biology of Neurons. Section 1: Handbook of Physiology, Part 1, Vol. 1. Bethesda, MD: American Physiological Society, 1977, pp. 39-97. 
[10] Tung, L., "A bi-domain model for describing ischemic myocardial D-C potentials." MIT, Cambridge, 1978.

[11] Roth, B. J., "Electrical conductivity values used with the bidomain model of cardiac tissue." IEEE Trans Biomed Eng, vol. 44, no. 4, pp. 326-328, 1997.

[12] Roth, B. J., "How the anisotropy of intracellular and extracellular conductivities influences stimulation of cardiac muscle." J Math Biol, vol. 30, no. 6, pp. 633-646, 1992.

[13] Wikswo, J. P., Jr., Lin, S.-F., and Abbas, R. A., "Virtual electrodes in cardiac tissue: A common mechanism for anodal and cathodal stimulation." Biophys J, vol. 69, no. 6, pp. 2195-2210, 1995.

[14] Henriquez, C. S., "Simulating the electrical behavior of cardiac tissue using the bidomain model." Crit Rev Biomed Eng, vol. 21, no. 1, pp. 1-77, 1993.

[15] McWilliam, J. A., "Fibrillar contractions of the heart." J Physiol (Lond), vol. 8 pp. 296$310,1887$.

[16] el-Sherif, N., "Reentrant mechanisms in ventricular arrhythmias.," in Zipes, D. P. and Jalife, J. (eds.) Cardiac Electrophysiology: From Cell to Bedside 2nd ed. Philadelphia, PA: W.B. Saunders Co, 1995, pp. 567-582.

[17] Mines, G. R., "On circulating excitations in heart muscles and their possible relation to tachycardia and fibrillation." Trans R Soc Can., vol. 4 pp. 43-52, 1914.

[18] Garrey, W. E., "Auricular fibrillation." Physiol Rev, vol. 4 pp. 215-250, 1924.

[19] Peters, N. S., Coromilas, J., Hanna, M. S., Josephson, M. E., Costeas, C., and Wit, A. L., "Characteristics of the temporal and spatial excitable gap in anisotropic reentrant circuits causing sustained ventricular tachycardia." Circ Res, vol. 82, no. 2, pp. 279-293, 1998.

[20] Hanna, M. S., Coromilas, J., Josephson, M. E., Wit, A. L., and Peters, N. S., "Mechanisms of resetting reentrant circuits in canine ventricular tachycardia." Circulation, vol. 103, no. 8, pp. 1148-1156, 2001.

[21] Hoffman, B. F. and Rosen, M. R., "Cellular mechanism for cardiac arrhythmias." Circ Res, vol. 49, no. 1, pp. 1-15, 1981.

[22] Allessie, M. A., Bonke, F. I. M., and Schopman, F. J. G., "Circus movement in rabbit atrial muscle as a mechanism of tachycardia: III. The "leading circle" concept. A new mode of circus movement in cardiac tissue witout the involvement of an anatomical obstacle." Circ Res, vol. 41 pp. 9-18, 1977.

[23] Pertsov, A. M., Davidenko, J. M., Salomonsz, R., Baxter, W. T., and Jalife, J., "Spiral waves of excitation underlie reentrant activity in isolated cardiac muscle." Circ Res, vol. 72, no. 3, pp. 631-650, 1993. 
[24] Girouard, S. D., Pastore, J. M., Laurita, K. R., Gregory, K. W., and Rosenbaum, D. S., "Optical mapping in a new guinea pig model of ventricular tachycardia reveals mechanisms for multiple wavelengths in a single reentrant circuit." Circulation, vol. 93, no. 3, pp. 603-613, Feb.1996.

[25] Chemical Waves and Patterns. Kapral, R. and Showalter, K. (eds.) Dordrecht: Kluwer Academic Publishers, 1995.

[26] Jakubith, S., Rotermund, H. H., Engel, W., von Oertzen, A., and Ertl, G., "Spatiotemporal concentration patterns in a surface reaction: Propagating and standing waves, rotating spirals, and turbulence." Phys Rev Lett, vol. 65, no. 24, pp. 3013-3016, 1990.

[27] Törnkvist, O. and Schröder, E., "Vortex dynamics in dissipative systems." Phys Rev Lett, vol. 78, no. 10, pp. 1908-1911, 1997.

[28] Levine, H., Aranson, I., Tsimring, L., and Truong, T. V., "Positive genetic feedback governs cAMP spiral wave formation in Dictyostelium." Proc Natl Acad Sci USA, vol. 93, no. 13, pp. 6382-6386, 1996.

[29] Ikeda, T., Uchida, T., Hough, D., Lee, J. J., Fishbein, M. C., Mandel, W. J., Chen, P.-S., and Karagueuzian, H. S., "Mechanism of spontaneous termination of functional reentry in isolated canine right atrium. Evidence for the presence of an excitable but nonexcited core." Circulation, vol. 94, no. 8, pp. 1962-1973, 1996.

[30] Weiner, N. and Rosenblueth, A., "The mathematical formulation of the problem of conduction of impulses in a network of connected excitable elements, specifically in cardiac muscle." Arch Inst Cardiol Mexico, vol. 16 pp. 205-265, 1946.

[31] Winfree, A. T., "Sudden cardiac death: A problem in topology." Scientific American, vol. 248, no. 5, pp. 144-161, 1983.

[32] Winfree, A. T., When Time Breaks Down: The Three-Dimensional Dynamics Of Electrochemical Waves And Cardiac Arrhythmias. Princeton: Princeton University Press, 1987.

[33] Frazier, D. W., Wolf, P. D., Wharton, J. M., Tang, A. S., Smith, W. M., and Ideker, R. E., "Stimulus-induced critical point. Mechanism for electrical initiation of reentry in normal canine myocardium." J Clin Invest, vol. 83, no. 3, pp. 1039-1052, 1989.

[34] el-Sherif, N., Gough, W. B., Zeiler, R. H., and Hariman, R., "Reentrant ventricular arrhythmias in the late myocardial infarction period. 12. Spontaneous versus induced reentry and intramural versus epicardial circuits." J Amer Coll Cardiol, vol. 6, no. 1, pp. 124-132, 1985.

[35] Davidenko, J. M., Pertsov, A., Salomonsz, R., Baxter, W., and Jalife, J., "Stationary and drifting spiral waves of excitation in isolated cardiac-muscle." Nature, vol. 355, no. 6358, pp. 349-351, 1992. 
[36] Bonometti, C., Hwang, C., Hough, D., Lee, J. J., Fishbein, M. C., Karagueuzian, H. S., and Chen, P.-S., "Interaction between strong electrical stimulation and reentrant wavefronts in canine ventricular fibrillation." Circ Res, vol. 77, no. 2, pp. 407-416, 1995.

[37] Gray, R. A., Jalife, J., Panfilov, A., Baxter, W. T., Cabo, C., Davidenko, J. M., and Pertsov, A. M., "Nonstationary vortexlike reentrant activity as a mechanism of polymorphic ventricular tachycardia in the isolated rabbit heart." Circulation, vol. 91, no. 9, pp. 2454-2469, 1995.

[38] Banville, I., Gray, R. A., and Ideker, R. E., "Shock-induced figure-eight reentry in the whole heart: An optical mapping study." Circ Res, vol. 85, no. 8, pp. 742-752, 1999.

[39] Sepulveda, N. G., Roth, B. J., and Wikswo, J. P., Jr., "Current injection into a twodimensional anisotropic bidomain." Biophys J, vol. 55 pp. 987-999, 1989.

[40] Roth, B. J., Lin, S.-F., and Wikswo, J. P., Jr., "Unipolar stimulation of cardiac tissue." J Electrocardiol, vol. 31 Suppl pp. 6-12, 1998.

[41] Lin, S.-F., Roth, B. J., and Wikswo, J. P., Jr., "Quatrefoil reentry in myocardium: An optical imaging study of the induction mechanism." J Cardiovasc Electrophysiol, vol. 10, no. 4, pp. 574-586, 1999.

[42] Davidenko, J. M., Pertsov, A. M., Salomonsz, R., Baxter, W. P., and Jalife, J., "Spatiotemporal irregularities of spiral wave activity in isolated ventricular muscle." $J$ Electrocardiol, vol. 24 Suppl pp. 113-122, 1992.

[43] Winfree, A. T., "Varieties of spiral wave behavior: An experimentalist's approach to the theory of excitable media." Chaos, vol. 1, no. 3, pp. 303-334, 1991.

[44] Zykov, V. S., "Cycloidal circulation of spiral waves in excitable medium." Biofizika, vol. 31 pp. 862-865, 1986.

[45] Barkley, D., "Spiral meandering.," in Kapral, R. and Showalter, K. (eds.) Chemical Waves and Patterns Boston: Kuwer, 1995, pp. 163-190.

[46] Biktashev, V. N. and Holden, A. V., "Re-entrant activity and its control in a model of mammalian ventricular tissue." Proc R Soc Lond B, vol. 263 pp. 1373-1382, 1996.

[47] Efimov, I. R., Krinsky, V. I., and Jalife, J., "Dynamics of rotating vortices in the BeelerReuter model of cardiac tissue." Chaos, Solitons, \& Fractals, vol. 5, no. 3-4, pp. 513-526, 1995.

[48] Wellner, M., Pertsov, A. M., and Jalife, J., "Spiral drift and core properties." Phys Rev E, vol. 59, no. 5, pp. 5192-5204, 1999.

[49] Steinbock, O., Schütze, J., and Müller, S. C., "Electric-field-induced drift and deformation of spiral waves in an excitable medium." Phys Rev Lett, vol. 68, no. 2, pp. 248-251, 1992. 
[50] Grill, S., Zykov, V. S., and Müller, S. C., "Feedback-controlled dynamics of meandering spiral waves." Phys Rev Lett, vol. 75, no. 18, pp. 3368-3371, 1995.

[51] Fast, V. G. and Pertsov, A. M., "Shift and termination of functional reentry in isolated ventricular preparations with quinidine-induced inhomogeneity in refractory period." $J$ Cardiovasc Electrophysiol, vol. 3, no. 3, pp. 255-265, 1992.

[52] Zykov, V. S., Steinbock, O., and Müller, S. C., "External forcing of spiral waves." Chaos, vol. 4, no. 3, pp. 509-518, 1994.

[53] Mantel, R.-M. and Barkley, D., "Periodic forcing of spiral waves in excitable media." Phys Rev E, vol. 54, no. 5, pp. 4791-4802, 1996.

[54] Gauthier, D. J., Hall, G. M., Oliver, R. A., Dixon-Tulloch, E. G., Wolf, P. D., and Bahar, S., "Progress toward controlling in vivo fibrillating sheep atria using a nonlineardynamics-based closed-loop feedback method." Chaos, vol. 12, no. 3, pp. 952-961, 2002.

[55] Krinsky, V. I. and Agladze, K. I., "Interaction of rotating waves in an active chemical medium." Physica D, vol. 8, no. 1-2, pp. 50-56, 1983.

[56] Ermakova, E. A., Pertsov, A. M., and Shnol, E. E., "On the interaction of vortices in twodimensional active media." Physica D, vol. 40, no. 2, pp. 185-195, 1989.

[57] Wu, X.-G., Chee, M.-N., and Kapral, R., "Vortex dynamics in oscillatory chemical systems." Chaos, vol. 1, no. 4, pp. 421-434, 1991.

[58] Elphick, C. and Meron, E., "Dynamics of phase singularities in 2-dimensional oscillatingsystems." Physica D, vol. 53, no. 2-4, pp. 385-399, 1991.

[59] Aranson, I. S. and Kramer, L., "The world of the complex Ginzburg-Landau equation." Rev Mod Phys, vol. 74, no. 1, pp. 99-143, 2002.

[60] Aranson, I. S., Kramer, L., and Weber, A., "On the interaction of spiral waves in nonequilibrium media." Physica D, vol. 53, no. 2-4, pp. 376-384, 1991.

[61] Aranson, I. S., Kramer, L., and Weber, A., "Theory of interaction and bound states of spiral waves in oscillatory media." Phys Rev E, vol. 47, no. 5, pp. 3231-3241, 1993.

[62] Agladze, K. I. and Krinskii, V. I., "Multi-armed vortices in an active-chemical medium." Nature, vol. 296, no. 5856, pp. 424-426, 1982.

[63] Vasiev, B., Siegert, F., and Weijer, C., "Multiarmed spirals in excitable media." Phys Rev Lett, vol. 78, no. 12, pp. 2489-2493, 1997.

[64] Hakim, V. and Karma, A., "Theory of spiral wave dynamics in weakly excitable media: Asymptotic reduction to a kinematic model and applications." Phys Rev E, vol. 60, no. 5, pp. 5073-5105, 1999. 
[65] Zaritski, R. M. and Pertsov, A. M., "Stable spiral structures and their interaction in twodimensional excitable media." Phys Rev E, vol. 66, no. 6, pp. 066120, 2002.

[66] Wu, T.-J., Bray, M.-A., Ting, C.-T., and Lin, S.-F., "Stable bound pair of spiral waves in rabbit ventricles." J Cardiovasc Electrophysiol, vol. 13, no. 4, pp. 414, 2002.

[67] Aliev, R. R., Davydov, V. A., Toshinori, K., and Yamaguchi, T., "Long range interaction of vortices in a chemical active medium." Netsu Sokutei, vol. 24, no. 4, pp. 194-198, 1997.

[68] Ruiz-Villarreal, M., Gómez-Gesteira, M., and Pérez-Villar, V., "Drift of interacting asymmetrical spiral waves." Phys Rev Lett, vol. 78, no. 5, pp. 779-782, 1997.

[69] Ermakova, E. A., Krinskii, V. I., Panfilov, A. V., and Pertsov, A. M., "Interaction of helical and flat periodic autowaves in an active medium." Biophysics, vol. 31, no. 2, pp. 348-354, 1986.

[70] Hendrey, M., Ott, E., and Antonsen, T. M., Jr., "Effect of inhomogeneity on spiral wave dynamics." Phys Rev Lett, vol. 82, no. 4, pp. 859-862, 1999.

[71] Nam, K., Ott, E., Gabbay, M., and Guzdar, P. N., "Spiral wave dynamics in the complex Ginzburg-Landau equation with broken chiral symmetry." Physica D, vol. 118, no. 1-2, pp. 69-83, 1998.

[72] Xie, F., Qu, Z., Weiss, J. N., and Garfinkel, A., "Interactions between stable spiral waves with different frequencies in cardiac tissue." Phys Rev E, vol. 59, no. 2, pp. 2203-2205, 1999.

[73] Gray, R. A., Pertsov, A. M., and Jalife, J., "Spatial and temporal organization during cardiac fibrillation." Nature, vol. 392, no. 6671, pp. 75-78, 1998.

[74] Winfree, A. T., "Scroll-shaped waves of chemical activity in three-dimensions." Science, vol. 181, no. 4103, pp. 937-939, 1973.

[75] Fenton, F. and Karma, A., "Fiber-rotation-induced vortex turbulence in thick myocardium." Phys Rev Lett, vol. 81, no. 2, pp. 481-484, 1998.

[76] Fenton, F. H., Cherry, E. M., Hastings, H. M., and Evans, S. J., "Multiple mechanisms of spiral wave breakup in a model of cardiac electrical activity." Chaos, vol. 12, no. 3, pp. 852-892, 2002.

[77] Streeter, D. D., Powers, W. E., Ross, M. A., and Torrent-Guasp, F., "Three-dimensional fiber orientation in the mammalian left ventricular wall.," in Baan, J., Noordergraaf, A., and Raines, J. (eds.) Cardiovascular System Dynamics. Cambridge, MA: MIT Press, 1978, pp. 73-84. 
[78] Berenfeld, O. and Pertsov, A. M., "Dynamics of intramural scroll waves in threedimensional continuous myocardium with rotational anisotropy." J Theor Biol, vol. 199 pp. 383-394, 1999.

[79] Wellner, M., Berenfeld, O., and Pertsov, A. M., "Predicting filament drift in twisted anisotropy." Phys Rev E, vol. 61, no. 2, pp. 1845-1850, 2000.

[80] Panfilov, A. V. and Keener, J. P., "Re-entry in three-dimensional FitzHugh-Nagumo medium with rotational anistropy." Physica D, vol. 84, no. 3-4, pp. 545-552, 1995.

[81] Fenton, F. and Karma, A., "Vortex dynamics in three-dimensional continuous myocardium with fiber rotation: Filament instability and fibrillation." Chaos, vol. 8, no. 1, pp. 20-47, 1998.

[82] Rappel, W.-J., "Filament instability and rotational tissue anisotropy: A numerical study using detailed cardiac models." Chaos, vol. 11, no. 1, pp. 71-80, 2001.

[83] Winfree, A. T., "Electrical turbulence in three-dimensional heart muscle." Science, vol. 266, no. 5187, pp. 1003-1006, 1994.

[84] Gray, R. A. and Jalife, J., "Ventricular fibrillation and atrial fibrillation are two different beasts." Chaos, vol. 8, no. 1, pp. 65-78, 1998.

[85] Biktashev, V. N., "A three-dimensional autowave turbulence." Int J Bifurc Chaos, vol. 8, no. 4, pp. 677-684, 1998.

[86] Qu, Z., Xie, F., and Garfinkel, A., "Diffusion-induced vortex filament instability in 3dimensional excitable media." Phys Rev Lett, vol. 83, no. 13, pp. 2668-2671, 1999.

[87] Allessie, M. A., Schalij, M. J., Kirchhof, C. J., Boersma, L., Huybers, M., and Hollen, J., "Experimental electrophysiology and arrhythmogenicity. Anisotropy and ventricular tachycardia." Eur Heart J, vol. 10 Suppl E pp. 2-8, 1989.

[88] Kavanagh, K. M., Kabas, J. S., Rollins, D. L., Melnick, S. B., Smith, W. M., and Ideker, R. E., "High-current stimuli to the spared epicardium of a large infarct induce ventricular tachycardia." Circulation, vol. 85, no. 2, pp. 680-698, 1992.

[89] Biktashev, V. N., Holden, A. V., and Zhang, H., "Tension of organizing filaments of scroll waves." Phil Trans R Soc Lond A, vol. 347, no. 1685, pp. 611-630, 1994.

[90] Aliev, R. R. and Panfilov, A. V., "A simple two-variable model of cardiac excitation." Chaos, Solitons, \& Fractals, vol. 7, no. 3, pp. 293-301, 1996.

[91] Karma, A., "Spiral breakup in model equations of action potential propagation in cardiac tissue." Phys Rev Lett, vol. 71, no. 7, pp. 1103-1106, 1993.

[92] Packard, N. H., Crutchfield, J. P., Farmer, J. D., and Shaw, R. S., "Geometry from a time series." Phys Rev Lett, vol. 45, no. 9, pp. 712-715, 1980. 
[93] Takens, F., "Detecting strange attractors in turbulence.," in Rand, D. A. and Young, L.-S. (eds.) Dynamical Systems and Turbulence Berlin: Springer-Verlag, 1981, pp. 366-381.

[94] Abarbanel, H. D. I., Brown, R., Sidorwich, J. J., and Tsimring, L. S., "The analysis of observed chaotic data in physical systems." Rev Mod Phys, vol. 65 pp. 1331-1392, 1993.

[95] Fraser, A. M. and Swinney, H. L., "Independent coordinates for strange attractors from mutual information." Physical Review A, vol. 33, no. 2, pp. 1134-1140, 1986.

[96] Gray, R. A., Wikswo, J. P., Jr., Lin, S.-F., and Baudenbacher, F. Phase mapping using both transmembrane potential and calcium. Pacing and Clinical Electrophysiology 23(4 Part II), 608. 2000.

[97] Choi, B.-R., Burton, F., and Salama, G., "Cytosolic $\mathrm{Ca}^{2+}$ triggers early afterdepolarizations and Torsade de Pointes in rabbit hearts with type 2 long QT syndrome." J Physiol (Lond), vol. 543, no. 2, pp. 615-631, 2002.

[98] Johnson, P. L., Smith, W., Baynham, T. C., and Knisley, S. B., "Errors caused by combination of Di-4 ANEPPS and Fluo3/4 for simultaneous measurements of transmembrane potentials and intracellular calcium." Ann Biomed Eng, vol. 27, no. 4, pp. 563-571, 1999.

[99] Choi, B.-R. and Salama, G., "Simultaneous maps of optical action potentials and calcium transients in guinea-pig hearts: mechanisms underlying concordant alternans." J Physiol (Lond), vol. 529, no. 1, pp. 171-188, 2000.

[100] Mermin, N. D., "The topological theory of defects in ordered media." Rev Mod Phys, vol. 51, no. 3, pp. 591-648, 1979.

[101] Winfree, A. T., "Electrical instability in cardiac muscle: Phase singularities and rotors." $J$ Theor Biol, vol. 138 pp. 353-405, 1989.

[102] Pertsov, A. M., Wellner, M., Vinson, M., and Jalife, J., "Topological constraint on scroll wave pinning." Phys Rev Lett, vol. 84, no. 12, pp. 2738-2741, 2000.

[103] Gabbay, M., Ott, E., and Guzdar, P. N., "Reconnection of vortex filaments in the complex Ginzburg-Landau equation." Phys Rev E, vol. 58, no. 2, pp. 2576-2579, 1998.

[104] Fiedler, B. and Mantel, R.-M., "Crossover collision of scroll wave filaments." Documenta Mathematica, vol. 5 pp. 695-731, 2000.

[105] Winfree, A. T. and Strogatz, S. H., "Organizing centres for three-dimensional chemical waves." Nature, vol. 311, no. 5987, pp. 611-615, 1984.

[106] Winfree, A. T., "Persistent tangles of vortex rings in excitable media." Physica D, vol. 84, no. 1-2, pp. 126-147, 1995. 
[107] Welsh, B. J., Gomatam, J., and Burgess, A. E., "Three-dimensional chemical waves in the Belousov-Zhabotinskii reaction." Nature, vol. 304, no. 5927, pp. 611-614, 1983.

[108] Jahnke, W., Henze, C., and Winfree, A. T., "Chemical vortex dynamics in threedimensional excitable media." Nature, vol. 336, no. 6200, pp. 662-665, 1988.

[109] Pertsov, A., Vinson, M., and Müller, S. C., "Three-dimensional reconstruction of organizing centers in excitable chemical media." Physica D, vol. 63, no. 1-2, pp. 233 240, 1993.

[110] Mironov, S., Vinson, M., Mulvey, S., and Pertsov, A., "Destabilization of threedimensional rotating chemical waves in an inhomogeneous BZ reaction." J Phys Chem, vol. 100, no. 5, pp. 1975-1983, 1996.

[111] Aranson, I. S., Bishop, A. R., and Kramer, L., "Dynamics of vortex lines in the threedimensional complex Ginzburg-Landau equation: Instability, stretching, entanglement, and helices." Phys Rev E, vol. 57, no. 5, pp. 5276-5286, 1998.

[112] Nam, K., Ott, E., Guzdar, P. N., and Gabbay, M., "Stability of spiral wave vortex filaments with phase twists." Phys Rev E, vol. 58, no. 2, pp. 2580-2585, 1998.

[113] Rodriguez, J. D., Pismen, L. M., and Sirovich, L., "Motion of interacting defects in the Ginzburg-Landau model." Physical Review A, vol. 44, no. 12, pp. 7980-7984, 1991.

[114] Cohen, L. B., Salzberg, B. M., Davilla, H. V., Ross, W. N., Landowne, D., Waggoner, A. S., and Wang, C. H., "Changes in axon fluorescence during activity: Molecular probes of membrane potential." J Membr Biol, vol. 19 pp. 1-34, 1974.

[115] Salama, G. and Morad, M., "Merocyanine 540 as an optical probe of transmembrane electrical activity in the heart." Science, vol. 191, no. 4226, pp. 485-487, Feb.1976.

[116] Dillon, S. and Morad, M., "A new laser scanning system for measuring action potential propagation in the heart." Science, vol. 214, no. 4519, pp. 453-456, Oct.1981.

[117] Knisley, S. B., "Optical mapping of cardiac electrical stimulation." J Electrocardiol, vol. 30 Suppl pp. 11-18, 1998.

[118] Lin, S.-F., Abbas, R. A., and Wikswo, J. P., Jr., "High-resolution high-speed synchronous epifluorescence imaging of cardiac activation." Rev Sci Instrum, vol. 68, no. 1, pp. 213217, 1997.

[119] Dillon, S. M., "Optical recordings in the rabbit heart show that defibrillation strength shocks prolong the duration of depolarization and the refractory period." Circ Res, vol. 69, no. 3, pp. 842-856, 1991.

[120] Dillon, S. M., "Optical mapping.," in Shenasa, M., Borggrefe, M., Breithadt, G., Haverkamp, K., and Hindricks, G. (eds.) Cardiac Mapping New York: Futura Publishing Co, Inc., 1993, pp. 587-606. 
[121] Spach, M. S., Dolber, P. C., and Heidlage, J. F., "Influence of the passive anisotropic properties on directional differences in propagation following modification of the sodium conductance in human atrial muscle. A model of reentry based on anisotropic discontinuous propagation." Circ Res, vol. 62, no. 4, pp. 811-832, 1988.

[122] Grinvald, A., Lieke, E., Frostig, R. D., Gilbert, C. D., and Wiesel, T. N., "Functional architecture of cortex revealed by optical imaging of intrinsic signals." Nature, vol. 324, no. 6095, pp. 361-364, 1986.

[123] el-Sherif, N., Gough, W. B., and Restivo, M., "Reentrant ventricular arrhythmias in the late myocardial infarction period: 14. Mechanisms of resetting, entrainment, acceleration, or termination of reentrant tachycardia by programmed electrical stimulation." PACE, vol. 10, no. 2, pp. 341-371, 1987.

[124] Jones, J. L., Jones, R. E., and Milne, K. B., "Refractory period prolongation by biphasic defibrillator waveforms is associated with enhanced sodium current in a computer model of the ventricular action potential." IEEE Trans Biomed Eng, vol. 41, no. 1, pp. 60-68, 1994.

[125] McGill, K. C., Cummins, K. L., Dorfman, L. J., Berlizot, B. B., Leutkemeyer, K., Nishimura, D. G., and Widrow, B., "On the nature and elimination of stimulus artifact in nerve signals evoked and recorded using surface electrodes." IEEE Trans Biomed Eng, vol. 29, no. 2, pp. 129-137, 1982.

[126] Colavita, P. G., Wolf, P., Smith, W. M., Bartram, F. R., Hardage, M., and Ideker, R. E., "Determination of effects of internal countershock by direct cardiac recordings during normal rhythm." Am J Physiol, vol. 250, no. 5 Pt 2, pp. H736-H740, 1986.

[127] Morad, M. and Salama, G., "Optical probes of membrane potential in heart muscle." $J$ Physiol (Lond), vol. 292 pp. 267-295, 1979.

[128] Efimov, I. R., Cheng, Y., Van Wagoner, D. R., Mazgalev, T., and Tchou, P. J., "Virtual electrode-induced phase singularity: A basic mechanism of defibrillation failure." Circ Res, vol. 82, no. 8, pp. 918-925, 1998.

[129] Muller, W., Windisch, H., and Tritthart, H. A., "Fluorescent styryl dyes applied as fast optical probes of cardiac action potential." Eur Biophys J, vol. 14, no. 2, pp. 103-111, 1986.

[130] Salzberg, B. M., Obaid, A. L., and Benzanilla, F., "Microsecond response of a voltagesensitive merocyanine dye: fast voltage-clamp measurements on squid giant axon." Jpn $J$ Physiol, vol. 43 Suppl 1 pp. S37-S41, 1993. 


\title{
CHAPTER III
}

\section{Experimental And Theoretical Analysis Of Phase Singularity DyNAMICS IN CARDIAC TISSUE}

Mark-Anthony Bray ${ }^{1}$, Shien-Fong Lin ${ }^{2}$, Rubin R. Aliev ${ }^{2}$, Bradley J. Roth ${ }^{3}$, and John P. Wikswo ${ }^{2}$

\author{
${ }^{1}$ Department of Biomedical Engineering \\ ${ }^{2}$ Department of Physics and Astronomy, \\ Vanderbilt University, Nashville, TN 37235 \\ ${ }^{3}$ Department of Physics \\ Oakland University, Rochester, MI 48309
}

Portions of this manuscript have been published in:

Bray, M-A, S-F Lin, RR Aliev, BJ Roth, and JP Wikswo, Journal of Cardiovascular Electrophysiology,

Vol. 12, No. 6, pp. 716-722, June 2001.

(C) 2001 by Futura Publishing Company, Inc. 


\subsection{Abstract}

Quantitative analysis of complex self-excitatory wave patterns, such as cardiac fibrillation and other high-order reentry, require the development of new tools for identification and tracking of the most important features of the activation, such as phase singularities. Imageprocessing operations can be used to detect the phase singularity at the tip of a spiral wave. The phase space behavior of a spatiotemporal sequence of data may be reconstructed using timeseries analysis. The phase singularities are then localized efficiently by computing the topological charge density as the curl of the spatial phase gradient. We analyze the singularity interaction dynamics of both experimentally observed and numerically simulated instances of quatrefoil reentry, and find that the singularity behavior in the experimental preparations may be classified into three categories on the basis of how their separation changes with time. We conclude that topological charge densities may be calculated easily and efficiently to reveal phase singularity behavior. However, the differences between theoretical and experimental observations of singularity separation distances indicate the need for more sophisticated numerical models.

\subsection{Introduction}

Reentrant excitation, a phenomenon in which a wave of excitation repeatedly activates the same area of tissue independently of the natural cardiac rhythm, is believed to play a significant role in the initiation of lethal arrhythmias, such as ventricular tachycardia and fibrillation [1,2]. The reentrant circuit around which activation propagates can be defined by an anatomical obstacle, such as an infarction, or by heterogeneities of dynamic electrical properties of normal cardiac tissue, such as the time course of repolarization and recovery of excitability $[3,4]$. Under such circumstances, the circuit can manifest itself in the form of spiral waves [5]. These waves may remain stationary, drift, and even give rise to multiple, dynamic reentrant pathways [6,7]. The quantitative analysis of these complex spatiotemporal patterns of activation requires the development of new tools that allow the identification and tracking of the most important topological features of the patterns. Simple excitatory waves in non-linear dynamical systems, such as the spiral wave in the Belousov-Zhabotinsky (BZ) reaction [8] or in cardiac tissue, are typically analyzed by comparing experimental observations with the theoretical predictions of numerical models [9]. 
The spiral wave rotates about an organizing center, or core, which is thought to be an unexcited but excitable medium that defines the primary dynamic characteristics of the wave $[10,11]$. Within the core, there is a phase singularity, i.e., a region where the phase is undefined. The phase singularity may be described in terms of topological charge, $n_{t}$, $[12,13]$, defined as

$$
n_{t} \equiv \frac{1}{2 \pi} \oint_{c} \nabla \phi \cdot d \vec{\ell}
$$

where $\phi(\vec{r})$ is the local phase, and the line integral is taken over path $\vec{\ell}$ on a closed curve $c$ surrounding the topological defect. Even though the core cannot be easily distinguished from the surrounding active tissue, it is possible to observe and track the phase singularity. Hence, it is upon this element that we will focus our attention.

\subsection{Methods}

Theoretical studies of self-excitatory systems can examine the relationship of the pair of key excitatory and recovery variables in phase space, in which one variable is plotted against the second. In experimental studies, there is typically a single observable variable, such as the transmembrane potential in cardiac tissue, which we will call $V(\vec{r}, t)$. To allow us to analyze the system in phase space, we use time-series analysis to create a second variable, $V^{\prime}(\vec{r}, t)$, which is defined by time delay embedding of $\mathrm{V}$,

$$
V^{\prime}(\vec{r}, t) \equiv V(\vec{r}, t+\tau)
$$

where $\tau$ is the delay calculated as the first zero crossing of the autocorrelation of $V(\vec{r}, t)$, indicating linear independence [14]. From these two variables, we can represent the temporal behavior of an excitable element in phase space as a closed path, termed a phase trajectory. We can then define the local phase of this element, $\phi(r, t)$, in terms of angle, around the trajectory in phase-plane, referenced to a central point.

By defining the integrand of Eq. (3.1), the gradient of the phase, as a wave vector, $\vec{k}$,

$$
\vec{k}(\vec{r}, t) \equiv \nabla \phi(\vec{r}, t)=\frac{\partial}{\partial x} \phi(\vec{r}, t) \hat{i}+\frac{\partial}{\partial y} \phi(\vec{r}, t) \hat{j}
$$

we see that by evaluating $n_{t}$ for a small circular path of radius $a$ in Eq. (3.1), the topological charge $n_{t}$ is proportional to the curl of the wave vector. Specifically, we are concerned with the component perpendicular to the plane containing $c$, i.e., 


$$
(\nabla \times \vec{k}) \cdot \hat{z} \equiv \lim _{a \rightarrow 0} \frac{1}{\pi a^{2}} \oint_{c} \vec{k} \cdot d \vec{\ell}
$$

where $\vec{k}$ and $\overrightarrow{d \ell}$ are assumed to be restricted to the $x y$ plane. Since $\vec{k}$ is derived from the gradient of a scalar field, the curl of $\vec{k}$ is zero everywhere where $\phi$ is differentiable, except at the phase singularity itself, where $\phi$ is undefined. This integral may be computed by the following procedure. The wave vector $\vec{k}$ may be approximated from a discretized phase image consisting of pixels $\phi[m, n]$ by a finite difference operation in the $x$ and $y$ directions

$$
\begin{aligned}
& k_{x}[m, n]=\nabla \phi_{x}[m, n]=\phi[m+1, n]-\phi[m, n] \\
& k_{y}[m, n]=\nabla \phi_{y}[m, n]=\phi[m, n+1]-\phi[m, n]
\end{aligned}
$$

Absolute phase jumps greater than $\pi$ in adjacent elements are corrected by converting them to their $2 \pi$ complement. Computation of the line integral in Eq. (3.1) at location $[m, n]$ may be expressed as a convolution operation

$$
(\nabla \times \vec{k}) \cdot \hat{z} \propto \nabla_{x} \otimes k_{y}+\nabla_{y} \otimes k_{x}
$$

where $\otimes$ is the convolution operator, and

$$
\nabla_{x}=\left[\begin{array}{ccc}
-1 / 2 & 0 & +1 / 2 \\
-1 & 0 & +1 \\
-1 / 2 & 0 & +1 / 2
\end{array}\right] \text {, and } \nabla_{y}=\left[\begin{array}{ccc}
+1 / 2 & +1 & +1 / 2 \\
0 & 0 & 0 \\
-1 / 2 & -1 & -1 / 2
\end{array}\right]
$$

are the convolution kernels.

The procedure developed above was used to locate phase singularities within a fourfold symmetric reentrant pattern known as quatrefoil reentry $[15,16]$. Such a pattern has recently been demonstrated in cardiac tissue, and, in contrast to most reentrant arrhythmias, it can be used to create repeatable patterns of four closely interacting singularities. In this paper, we analyze data from our earlier experiments. The experimental protocol has been described previously [16], but we summarize it here briefly. High-speed optical imaging using a CCD camera at 267 frames per second was used to record the response of 5 isolated, Langendorff-perfused rabbit hearts stained with the voltage-sensitive dye di-4-ANEPPS. $1 \mu \mathrm{M}$ of the calcium channel antagonist D600 was added to the perfusate to inhibit motion artifacts. A premature S2 cathodal stimulus was delivered within the vulnerable phase during periodic pacing via a unipolar point electrode 
placed on the posterior left ventricle. Within a narrow range of coupling intervals, the boundary between the induced virtual anodes and virtual cathodes provided four pathways by which reentry could occur after the termination of the S2 stimulus. While the resultant quatrefoil reentry was not sustained, lasting only a few cycles, there was sufficient propagation to clearly identify the reentrant pattern. Spatial and temporal filtering was applied to improve the signal-tonoise ratio, and the signals were normalized with respect to the S1 amplitude. The value of $\tau$ used for a spatiotemporal data sequence was calculated as the average of the optimal values of $\tau$ calculated for each pixel in that sequence.

\subsection{Results}

The earlier work by Gray et al. [14] and our present study are the first that provide quantitative, experimental measurements of the interaction dynamics of multiple singularities in cardiac tissue. While Gray tracked as many as four singularities at one time and could observe singularity annihilation, the dynamics of the interaction between these singularities was not analyzed. In contrast, our experimental and theoretical studies of quatrefoil reentry provide us with the requisite data required to test our understanding of these interactions. For this study, the tissue was modeled as an anisotropic bidomain, both as a 2-D sheet of tissue and a 3-D cylinder of tissue, using cylindrical coordinates $(z, \rho, \theta)$ to specify position; axisymmetry allowed the results for the cylinder to be determined independently of $\theta$ by means of an appropriate coordinate transformation. The active membrane components were modeled using Beeler-Reuter kinetics, slightly modified such that strong hyperpolarization would not produce unrealistic membrane conductance, and the speed of the calcium channel kinetics increased by a factor of eight to ensure a stable spiral wave; further details on the model parameters are described elsewhere [17]. Figure 3.1 shows the spatiotemporal pattern described by quatrefoil reentry for both a numerically simulated model and an experimental preparation; both groups of data were normalized to unity with respect to the $\mathrm{S} 1$ stimulus.

Previous studies $[5,16]$ have indicated that a reduced variation in action potential amplitude exists in those regions which are either undergoing conduction block or are located 


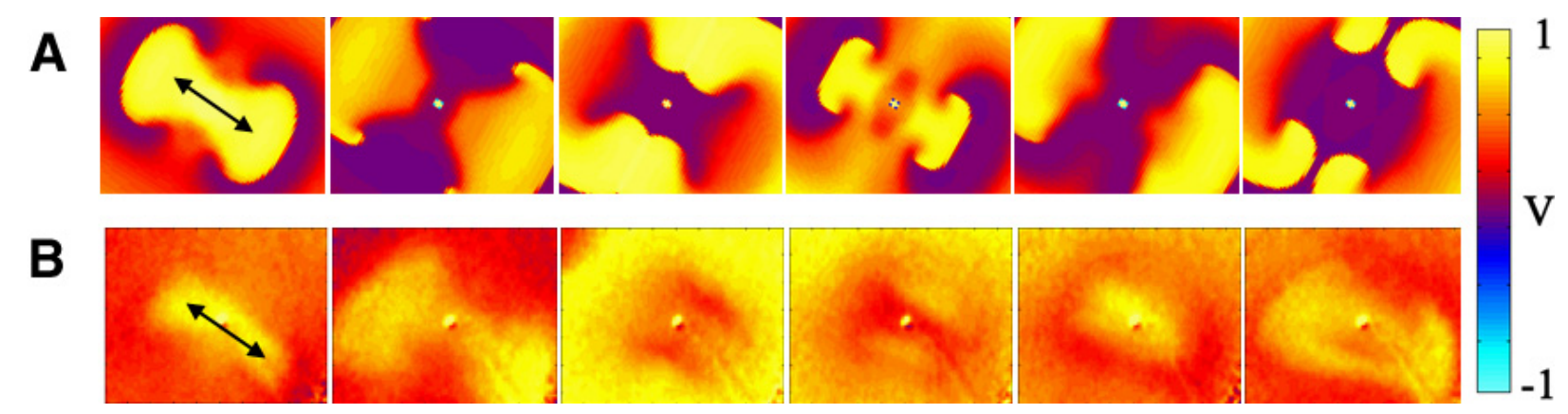

Figure 3.1: Images of the spatiotemporal distribution during quatrefoil reentry of the (A) transmembrane potential in a numerically simulated model and (B) the fluorescence intensity in an experimental preparation. The fluorescent signal obtained from the epicardium is proportional to the transmembrane potential. The color bar indicates the pseudocolors used for representing the normalized signal magnitude; yellow is fully depolarized, blue is hyperpolarized, purple is resting. The arrow indicates fiber direction.

within the core of a spiral wave. It stands to reason that calculating the variance of sequential, overlapping segments of the temporal signal and identifying those regions of low variance will highlight these areas. Figure 3.2 shows the spatiotemporal relationship between the motion of the activation wave and the regions of low variance shortly after the initiation of quatrefoil reentry for both the numerical model and experimental preparation. In each instance, the black mesh encloses the low variance regions calculated with an isovalue of 0.2 in Fig. 3.2A and 0.06 in 3.2B. The presence of four distinct low variance regions and the fact that the colored surface representing the wavefront rotates around each of the meshes confirms that the phase singularity should be located within the low-variance region.

Figure 3.3 displays a comparison between numerical simulation and experimental preparations of quatrefoil reentry, along with the images after application of the phase and curl operations. The calculated values of $\tau$ were on the order of $26 \mathrm{~ms}$ for the experimental data sequences and $35 \mathrm{~ms}$ for the numerically simulated data sets. In Fig. 3.3B and C, note the presence of four phase singularities; in particular, the value of the curl at the singularity positions in Fig. 3.3C corresponds to the spiral wave chirality (blue corresponds to clockwise rotation, red to counter-clockwise). Thus, by this method, we succeed in isolating the phase singularity of spiral waves for analysis. Figure 3.4 shows an example of quatrefoil trajectories obtained from application of the above algorithm to both experimentally prepared and numerically simulated data. It can clearly be seen that the singularities exhibit dynamic behavior. 

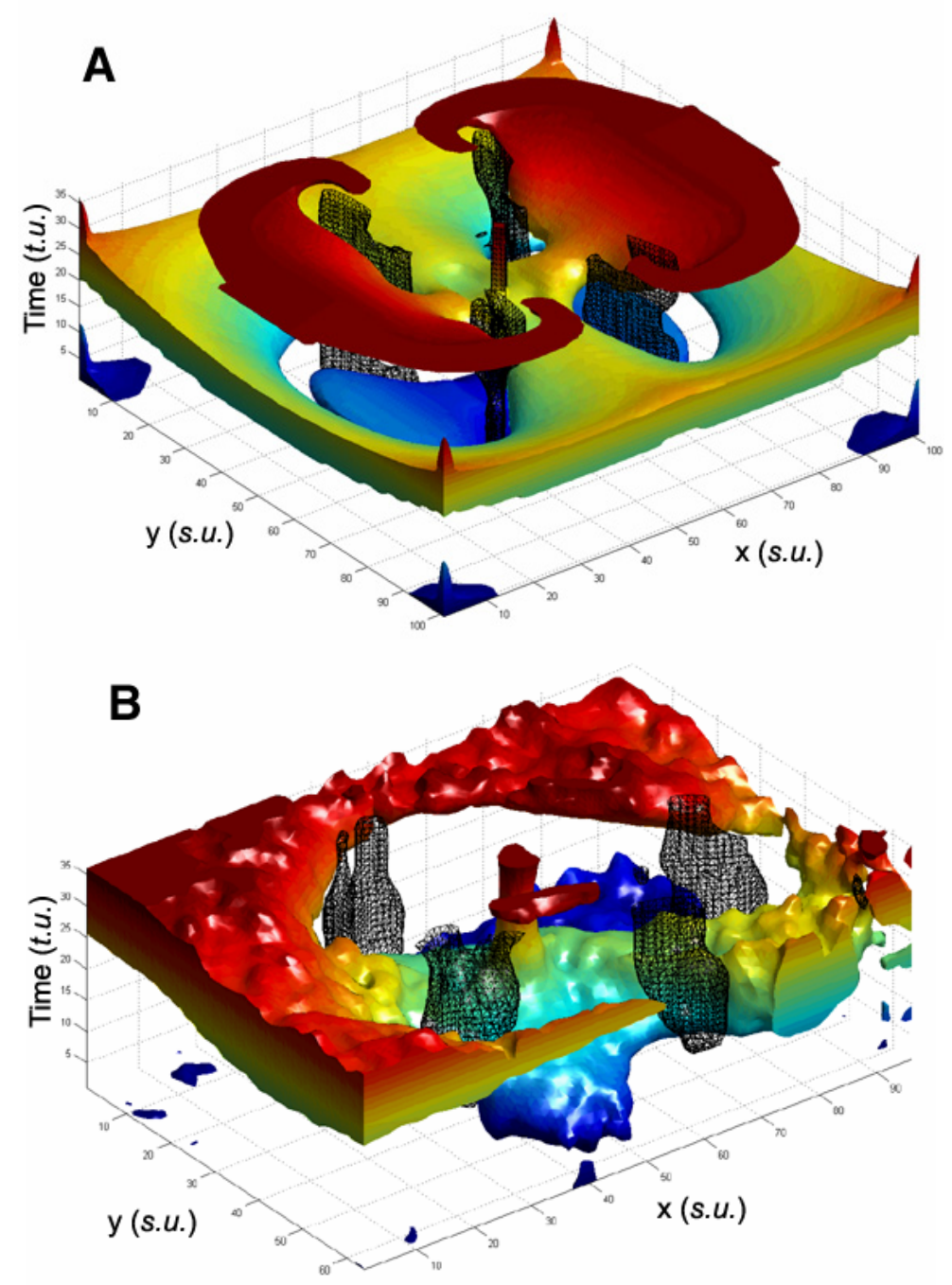

Figure 3.2: Depiction of the spatiotemporal evolution of a quatrefoil reentrant wavefront pattern relative to regions of low variance of wavefront amplitude in (A) numerically simulated and (B) experimental preparations. Spatial axes lies in the horizontal plane, temporal axis is vertical; spatial units in s.u., time units in $t . u$. Solid surface represents isopotential surface of the reentrant wavefront where the normalized $V_{m}$ is 0.7 , colored for clarity. Black mesh encloses regions of reduced variance in temporal signal, calculated at a threshold variance value of 0.2 in (A), 0.06 in (B).

In three-dimensional figure-of-eight reentry, the symmetry of the system requires that the two singularities define the ends of a filament about which the single scroll wave rotates. In the instance of quatrefoil reentry, the observed surface pattern is consistent with two pairs of phase singularities connected by two singularity filaments, about which a pair of synchronized scroll 
waves rotates underneath the epicardial surface. If we consider the finite thickness of the left ventricular wall, the possibility of two transmural filaments cannot be ruled out conclusively. For this paper, we define the term transverse pair, TP, as the oppositely charged singularity pair aligned perpendicular to the axis of symmetry defined by the cardiac fiber direction, as shown in Fig. 3.3A. A longitudinal pair, LP, describes the pair of singularities aligned parallel to the cardiac fiber axis of symmetry. Examples of TP and LP are illustrated in experimental and simulated data in Fig. 3.3C.

We examined relative distances between transverse and longitudinal singularity pairs for twenty cases of quatrefoil reentry. For the earliest detectable singularities, in the majority of cases the TP separation was less than that of the LP separation. We found that transverse and longitudinal pairs exhibit dynamic behavior which could be grouped into three classifications on the basis of the time course of the average pair separation, as shown by specific examples of trajectories in Fig. 3.4. The average of the TP and LP separation distances for each of the 20 instances of quatrefoil reentry is shown in Fig. 3.5. Figure 3.6 depicts the TP and LP separation distances, along with the average of the TP and LP separation distances, averaged for each classification. The taxonomy of the separation distances is described as follows:

1. Type $I(n=10)$ is characterized by an initial expansion in the TP separation distance and a contraction in the LP separation distance to form a more equidistant arrangement in the four singularities. The initial phase has an average expansion velocity of $0.20 \mathrm{~mm} / \mathrm{ms}$. Both pairs then exhibit weak oscillatory behavior at a relatively constant separation distance (Fig. 3.4A, 3.5A, 3.6A).

2. Type II $(n=4)$ is characterized by an initial overall contraction in both TP and LP separation distance, with an average contraction velocity of $0.30 \mathrm{~mm} / \mathrm{ms}$. Thereafter, both TP and LP separation distances experience strong oscillatory behavior, coupled with a contraction in separation distance (Fig. 3.4B, 3.5B, 3.6B).

3. Type III $(n=6)$ is characterized by an initial overall expansion in both TP and LP separation distances, with an average expansion velocity of $0.83 \mathrm{~mm} / \mathrm{ms}$. Afterward, both pairs then exhibit oscillatory behavior with an expanding trend in separation distance. At times, the average TP separation is less than half that of the average LP separation (Fig. 3.4C, 3.5C, $3.6 \mathrm{C})$. 

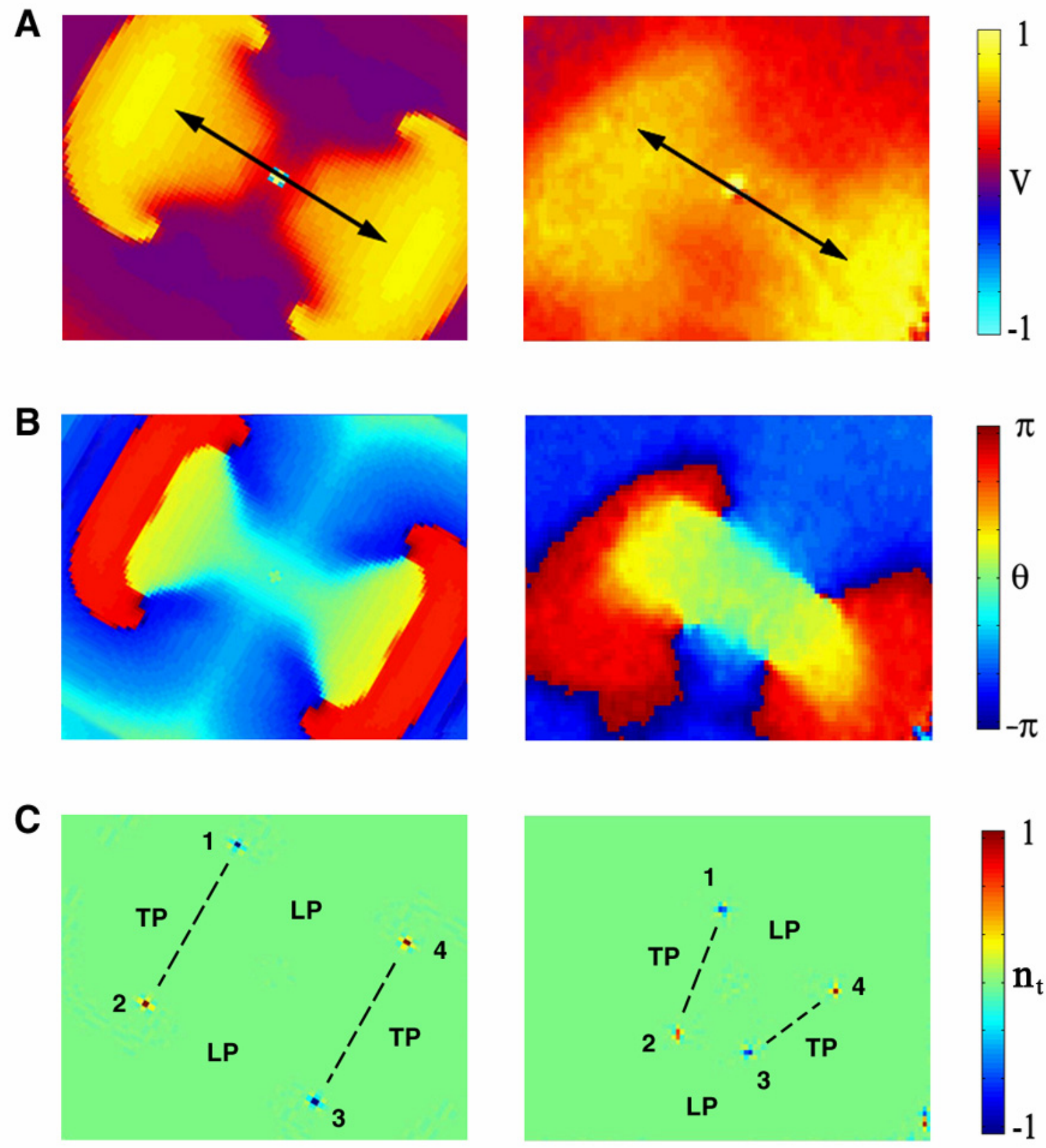

Figure 3.3: Numerically simulated (left column) and experimental (right column) quatrefoil reentry following an electrical stimulus applied to the center of tissue. (A) Normalized spatial distribution of transmembrane potential from simulated data, and fluorescence intensity from experimental data. The arrow indicates fiber direction. (B) Phase distribution of image in (A). (C) Curl distribution of wave vectors calculated from image in (B), highlighting the presence of four phase singularities. The transverse pairs (TP) are indicated by numbers 1 and 2, and 3 and 4 , the longitudinal pairs (LP) indicated by numbers 1 and 4, and 2 and 3. 

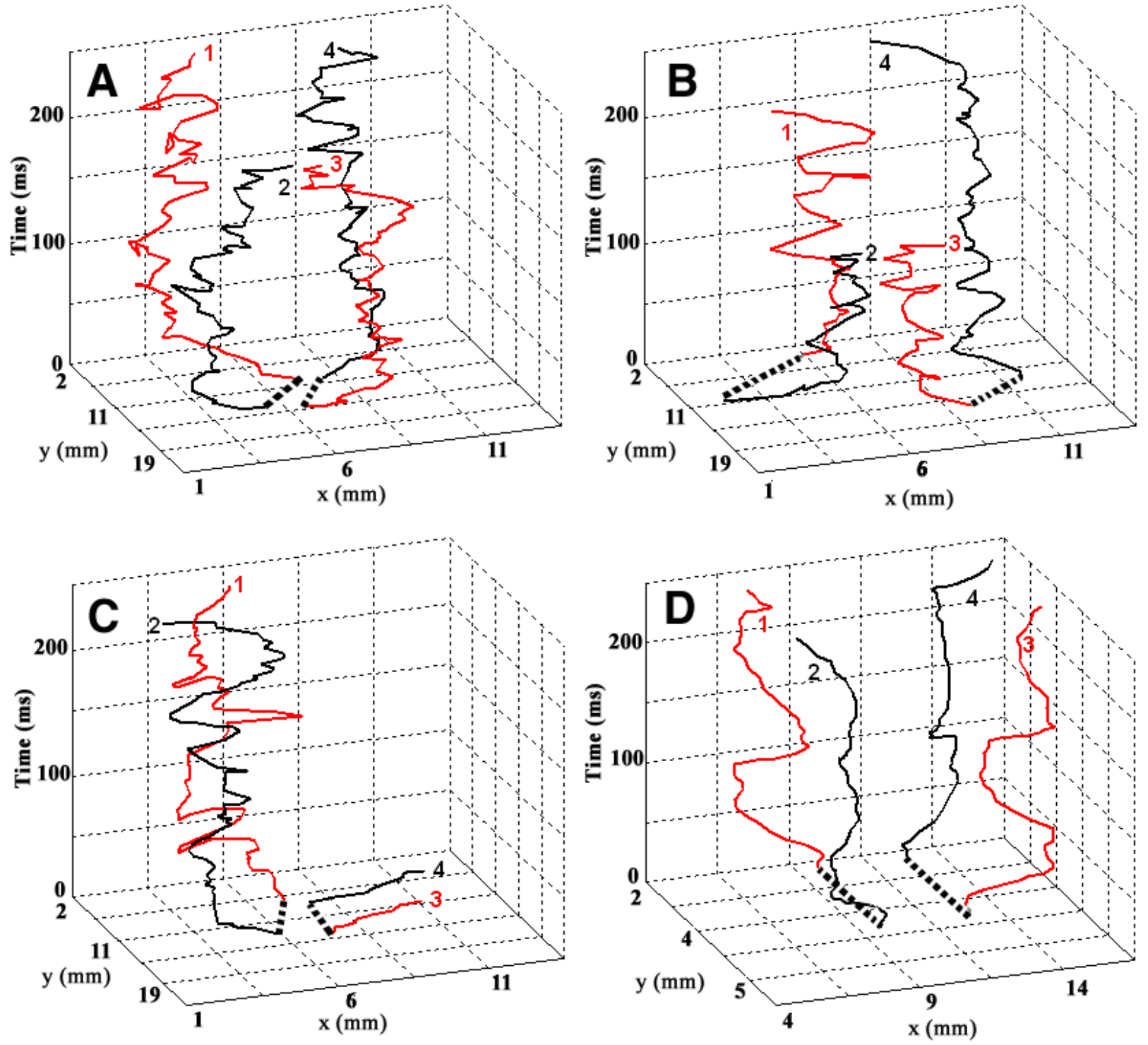

Figure 3.4: Demonstration of trajectories of quatrefoil phase singularities in $(x, y, t)$ space, illustrating dynamic behavior in time. The singularities are numbered (1-4) and connected in the same order as in Fig. 3.3, to indicate TP and LP. Time interval is measured from the end of the S2 stimulus. (A) Type I: initial linear expansion followed by linear contraction; (B) Type II: initial linear contraction followed by oscillatory contraction; (C) Type III: initial linear expansion followed by expansion trend; (D) 3-D Numerical model pair separation distances. 

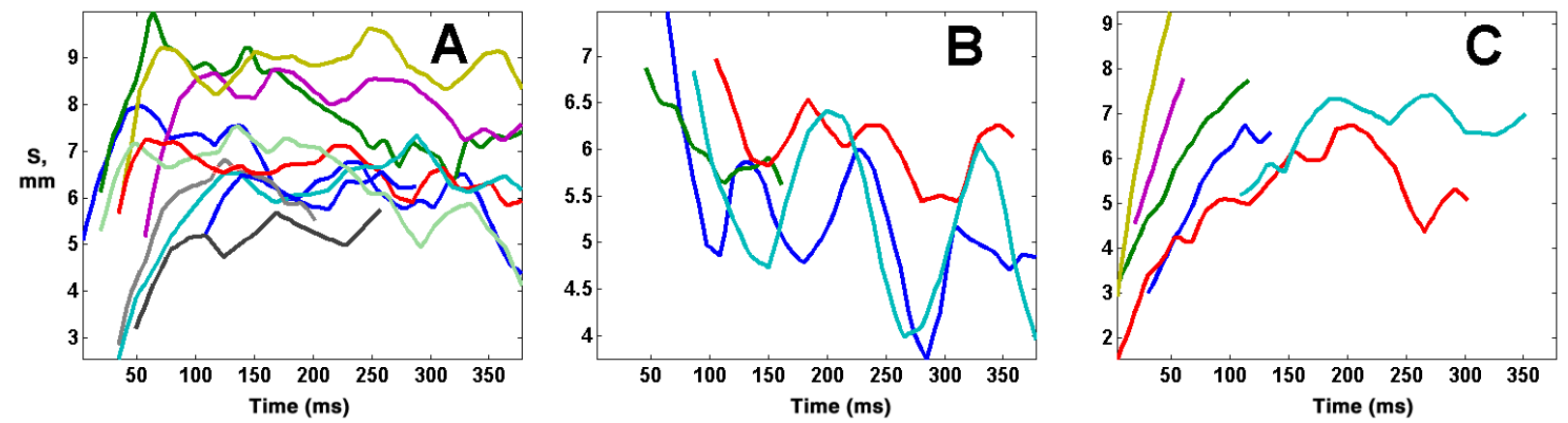

Figure 3.5: Plots of the average of the TP and LP separation distances for all instances of quatrefoil reentry grouped into the three types. Each curve represents a different experiment. (A) Type I; (B) Type II; (C) Type III.
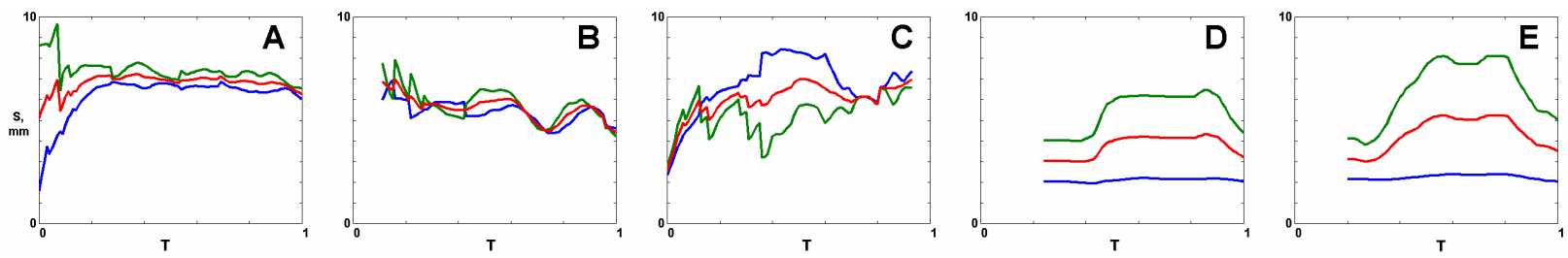

Figure 3.6: Taxonomy of the pair separation distance $\mathrm{S}$ (in $\mathrm{mm}$ ) plotted as a function of time $\mathrm{T}$, computed as a fraction of the rotation period. $\mathrm{T}=0$ represents the termination of the $\mathrm{S} 2$ stimulus. Blue, green, and red lines represent TP, LP, and average separation distances, respectively. Curves begin from time of first detection of singularities. (A) Type I; (B) Type II; (C) Type III; (D) 2-D Numerical model pair separation distances; (E) 3-D Numerical model pair separation distances.

Several interesting observations may be made on the basis of Fig. 3.4. Note that in C, a TP is the first to extinguish (labeled 3 and 4). However, in A and B, the LP singularities labeled 2 and 3 are extinguished in both cases, most likely by mutual annihilation in the instance of A, on the basis of their proximity at the time the singularities disappear. This behavior in A and B suggests that the filament remnants of 1 and 4 have either spanned the myocardial wall so that the other end of the filament is not visible, or recombined to form a new transverse pair [18].

The same calculations were applied to the numerically simulated data. In comparison, analysis of pair separation for the model data sets did not conform to any of the above classifications, exhibiting clearly repetitive oscillatory behavior for both TP and LP, in both the two- and three-dimensional cases. Examples of numerically simulated separation distances are shown in Fig. 3.6D and E. 

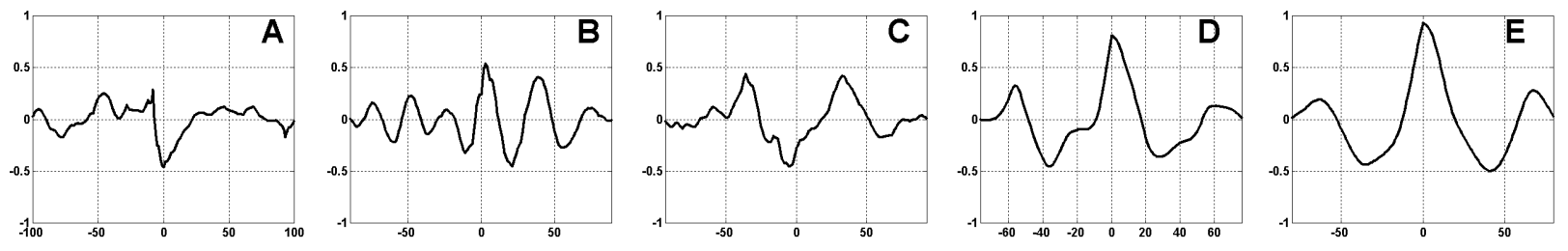

Figure 3.7: Cross-correlation of TP and LP separation distances. (A) Type I; (B) Type II; (C) Type III; (D) 2-D Numerical model; (E) 3-D Numerical model.

Examination of the periodicity of the interaction between the singularities was performed by cross-correlating the TP and LP separation distances, as shown in Fig. 3.7. As indicated above, the oscillatory nature of the Type II behavior is reflected in the cross correlation (Fig. 3.7B) which indicated a period of oscillation of $133 \mathrm{~ms}$. Type III also suggested an oscillation of separation distances (Fig. 3.7C), albeit over a wider period, unlike Type I, which exhibited no interdependence of separation distances (Fig. 3.7A). The cross-correlation for the numerical models both display a strong correlation at the zero-th shift.

\subsection{Discussion}

Now that we have identified the phase singularities and can compute their trajectory in physical space, we can return to Figure 3.2 and compare the trajectory to the regions of low variance. Figure 3.8 shows a single spiral wave from the quatrefoil reentry with the corresponding phase singularity trajectory overlaid as a white line. While the mesh encloses the trajectory completely in the numerical model (Fig. 3.8A), for the instance of the experimental preparation, the trajectory may wander outside the mesh at some places (Fig. 3.8B). Whether this disparity is due to the rapidity of singularity motion, the width of the overlapping temporal segments used to calculate variance, or the calculation of phase itself is a topic of further research. In both cases, we see that phase singularity trajectory follows the time course of the low variance mesh, as expected.

While the interaction of rotors has been discussed intensively in the literature [19-21], the problem is far from being resolved. Analytical solutions have been found for simple systems such as those governed by the Ginzburg-Landau equations [22,23], demonstrating that inhomogeneities within the medium will cause frequency-dependent spiral wave interaction. For 

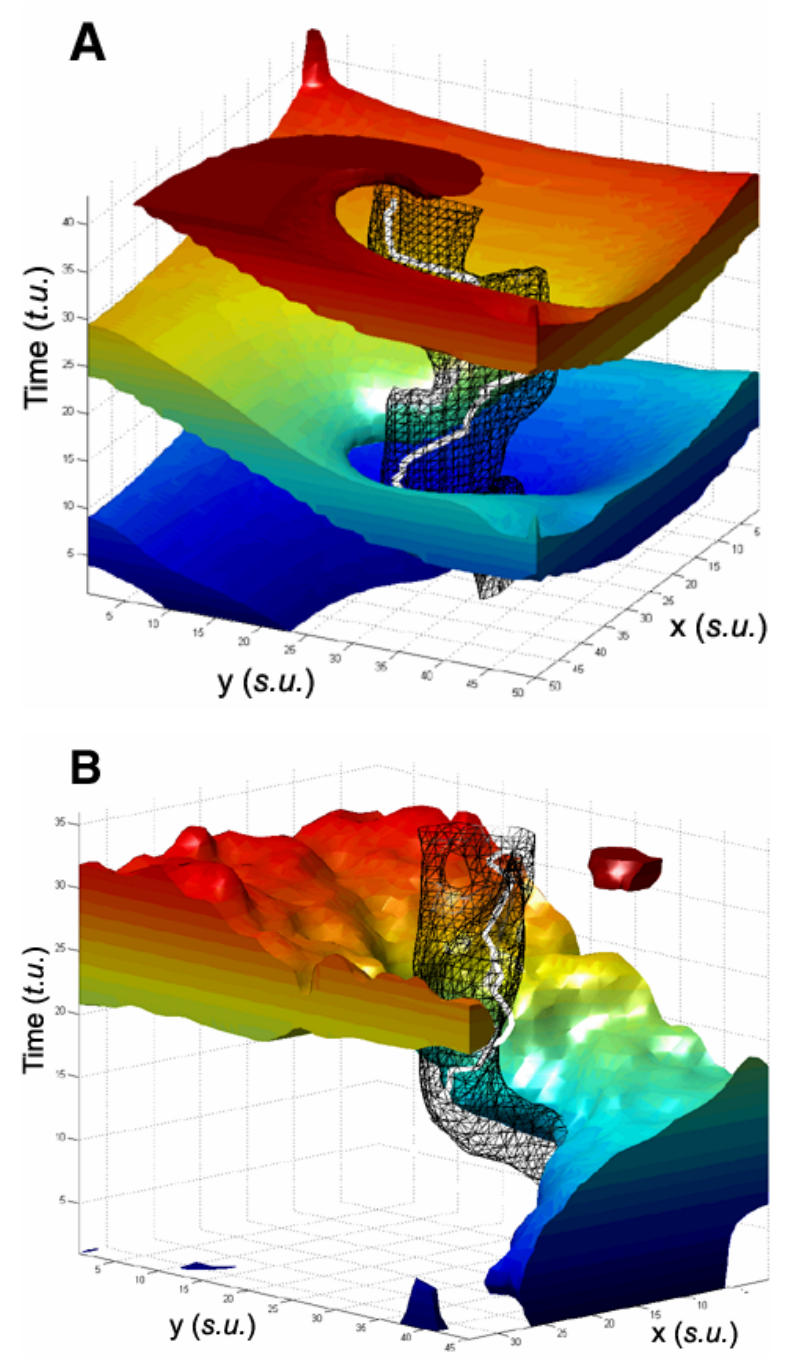

Figure 3.8: Depiction of the spatiotemporal evolution of a single spiral wave from Fig. 3.2 relative to region of low variance of wavefront amplitude and phase singularity trajectory in (A) numerically simulated and (B) experimental preparations. Spatial axis lies in the horizontal plane, temporal axis in vertical plane; spatial units in s.u., time units in t.u.. Solid surface represents isopotential surface of the reentrant wavefront where the normalized $V_{m}$ is 0.7 , colored for clarity. Black mesh encloses regions of reduced variance in temporal signal, calculated at a threshold variance value of 0.2 in (A), 0.06 in (B). Trajectory of the phase singularity represented as a thick white line.

systems with FitzHugh-Nagumo dynamics, no analytic solution has yet been found; numerical simulations show the oscillatory interaction of vortices at close range where the parameter values have been constrained to prohibit single vortex meander [24], a significant difference from experimental observations [6,7]. The long-range interaction of vortices has been studied in the $\mathrm{BZ}$ reaction [25], again indicating a frequency-dependent element involved in vortex drift. At 
short distances, it has been experimentally established that vortices with opposite topological charges can annihilate [14], in accordance with theory [13].

In this paper, we have observed a significant difference in the singularity dynamics (as defined as the time dependence of the singularity separation distance) between the experimental preparations and numerical models. One possible source of this discrepancy may be the addition of the excitation decoupler D600 to suppress mechanical contractions for the purposes of the experiment. The use of electromechanical uncoupling agents have been used previously for the purpose of optical mapping of phase singularities [14]. D600 is known to alter wavefront dynamics, but its full range of effects on singularity dynamics cannot be determined without further study beyond the scope of this initial paper.

Current evidence supports the dependence of epicardial phase singularity dynamics on underlying filament behavior [7]. Further exploration into the correlation of experimental and numerical results will necessitate the development of more detailed models. A future step is to carry out numerical simulations of filament behavior in a 3-D model of continuous myocardium which more closely represents the physiological substrate that may be found in experimental preparations. Another issue which needs to be addressed is the fact that using time series analysis is but one of several techniques to calculate phase for an oscillatory system (for a review, see Pikovsky et al. [26]). The algorithm described here is designed to detect a $2 \pi$ phase change around a point, regardless of the technique used to generate the spatial distribution of phase values. However, the influence of the method chosen to construct the phase map on phase singularity localization (and the derived dynamics) is not yet fully understood.

\subsection{Conclusion}

The concept of topological charge provides a technique to localize phase singularities easily and efficiently present in cardiac tissue that is undergoing reentrant excitation to reveal the dynamics of their behavior. Our experimental study demonstrates both expansion and contraction of the distance between singularities, and annihilation of singularities that are either connected by a filament or not. We conclude that the experimentally observed dynamics are more complex and varied than those predicted our model, either because the dynamical properties of the model are too simple, or the anisotropies and heterogeneities of the tissue are not adequately represented, or the model is not operating in the correct parameter space. Until the models are 
improved, our experimental observations of quatrefoil reentry provide us with an excellent, although experimentally challenging, system for the study of singularity dynamics.

\subsection{Acknowledgments}

This work has been supported in part by NIH grants R01HL58241, R01HL57207 and a National Science Foundation predoctoral fellowship to Mark-Anthony Bray. We are indebted to Richard Gray and Alan Garfinkel for their comments and suggestions.

\section{8. $\quad$ References}

[1] Mines, G. R., "On circulating excitations in heart muscles and their possible relation to tachycardia and fibrillation." Trans $R$ Soc Can., vol. 4 pp. 43-52, 1914.

[2] el-Sherif, N., "Reentrant mechanisms in ventricular arrhythmias.," in Zipes, D. P. and Jalife, J. (eds.) Cardiac Electrophysiology: From Cell to Bedside 2nd ed. Philadelphia, PA: W.B. Saunders Co, 1995, pp. 567-582.

[3] Allessie, M. A., Bonke, F. I. M., and Schopman, F. J. G., "Circus movement in rabbit atrial muscle as a mechanism of tachycardia: I." Circ Res, vol. 23 pp. 54-62, 1973.

[4] Hoffman, B. F. and Rosen, M. R., "Cellular mechanism for cardiac arrhythmias." Circ Res, vol. 49, no. 1, pp. 1-15, 1981.

[5] Pertsov, A. M., Davidenko, J. M., Salomonsz, R., Baxter, W. T., and Jalife, J., "Spiral waves of excitation underlie reentrant activity in isolated cardiac muscle." Circ Res, vol. 72, no. 3, pp. 631-650, 1993.

[6] Davidenko, J. M., Pertsov, A. M., Salomonsz, R., Baxter, W. P., and Jalife, J., "Spatiotemporal irregularities of spiral wave activity in isolated ventricular muscle." $J$ Electrocardiol, vol. 24 Suppl pp. 113-122, 1992.

[7] Gray, R. A., Jalife, J., Panfilov, A., Baxter, W. T., Cabo, C., Davidenko, J. M., and Pertsov, A. M., "Nonstationary vortexlike reentrant activity as a mechanism of polymorphic ventricular tachycardia in the isolated rabbit heart." Circulation, vol. 91, no. 9, pp. 2454-2469, 1995.

[8] Zaikin, A. N. and Zhabotinsky, A. M., "Concentration wave propagation in twodimensional liquid-phase self-oscillating system." Nature, vol. 225 pp. 535-537, 1970.

[9] Winfree, A. T., When Time Breaks Down: The Three-Dimensional Dynamics Of Electrochemical Waves And Cardiac Arrhythmias. Princeton: Princeton University Press, 1987. 
[10] Zykov, V. S., Simulation of Wave Processes in Excitable Media. Manchester: Manchester University Press, 1987.

[11] Ikeda, T., Uchida, T., Hough, D., Lee, J. J., Fishbein, M. C., Mandel, W. J., Chen, P.-S., and Karagueuzian, H. S., "Mechanism of spontaneous termination of functional reentry in isolated canine right atrium. Evidence for the presence of an excitable but nonexcited core." Circulation, vol. 94, no. 8, pp. 1962-1973, 1996.

[12] Goryachev, A. and Kapral, R., "Spiral waves in chaotic systems." Phys Rev Lett, vol. 76, no. 10, pp. 1619-1622, 1996.

[13] Mermin, N. D., "The topological theory of defects in ordered media." Rev Mod Phys, vol. 51, no. 3, pp. 591-648, 1979.

[14] Gray, R. A., Pertsov, A. M., and Jalife, J., "Spatial and temporal organization during cardiac fibrillation." Nature, vol. 392, no. 6671, pp. 75-78, 1998.

[15] Roth, B. J., Lin, S.-F., and Wikswo, J. P., Jr., "Unipolar stimulation of cardiac tissue." J Electrocardiol, vol. 31 Suppl pp. 6-12, 1998.

[16] Lin, S.-F., Roth, B. J., and Wikswo, J. P., Jr., "Quatrefoil reentry in myocardium: An optical imaging study of the induction mechanism." J Cardiovasc Electrophysiol, vol. 10, no. 4, pp. 574-586, 1999.

[17] Roth, B. J., "Nonsustained reentry following successive stimulation of cardiac tissue through a unipolar electrode." J Cardiovasc Electrophysiol, vol. 8, no. 7, pp. 768-778, 1997.

[18] Fenton, F. and Karma, A., "Fiber-rotation-induced vortex turbulence in thick myocardium." Phys Rev Lett, vol. 81, no. 2, pp. 481-484, 1998.

[19] Krinsky, V. I. and Agladze, K. I., "Interaction of rotating waves in an active chemical medium." Physica D, vol. 8, no. 1-2, pp. 50-56, 1983.

[20] Wu, X.-G., Chee, M.-N., and Kapral, R., "Vortex dynamics in oscillatory chemical systems." Chaos, vol. 1, no. 4, pp. 421-434, 1991.

[21] Aranson, I. S., Kramer, L., and Weber, A., "On the interaction of spiral waves in nonequilibrium media." Physica D, vol. 53, no. 2-4, pp. 376-384, 1991.

[22] Hagan, P. S., "Spiral waves in reaction-diffusion equations." SIAM J Appl Math, vol. 42, no. 4, pp. 762-786, 1982.

[23] Hendrey, M., Ott, E., and Antonsen, T. M., Jr., "Effect of inhomogeneity on spiral wave dynamics." Phys Rev Lett, vol. 82, no. 4, pp. 859-862, 1999.

[24] Ermakova, E. A., Pertsov, A. M., and Shnol, E. E., "On the interaction of vortices in twodimensional active media." Physica D, vol. 40, no. 2, pp. 185-195, 1989. 
[25] Aliev, R. R., Davydov, V. A., Toshinori, K., and Yamaguchi, T., "Long range interaction of vortices in a chemical active medium." Netsu Sokutei, vol. 24, no. 4, pp. 194-198, 1997.

[26] Pikovsky, A. S., Rosenblum, M. G., Osipov, G. V., and Kurths, J., "Phase synchronization of chaotic oscillators by external driving." Physica D, vol. 104, no. 3-4, pp. 219-238, 1997. 


\title{
CHAPTER IV
}

\section{Considerations In Phase Plane Analysis For Non-Stationary REENTRANT CARDIAC BEHAVIOR}

\author{
Mark-Anthony Bray ${ }^{1}$ and John P. Wikswo ${ }^{2}$
}

\author{
${ }^{1}$ Department of Biomedical Engineering \\ ${ }^{2}$ Department of Physics and Astronomy \\ Vanderbilt University
}

Nashville, TN 37235

Portions of this manuscript have been published in:

Bray, M-A and JP Wikswo, Physical Review E

Vol. 65, No. 5, pp. 051902, May 2002.

(C) 2002 by the American Physical Society 


\subsection{Abstract}

Cardiac reentrant arrhythmias may be examined by using time-series analysis, where a state variable is plotted against the same variable with an embedded time delay, $\tau$ to form a phase portrait. The success of this procedure is contingent upon the resultant phase space trajectories encircling a fixed origin. However, errors in interpreting the dynamics of phase singularities associated with reentry may arise due to the trajectories not encircling the origin or due to a poor choice of $\tau$. We demonstrate an algorithm which is capable of establishing proper orbits without the need to specify $\tau$. We find that phase singularities could be localized closer to the point of initial formation than was possible previously, which is important for the purposes of singularity tracking and investigating electrodynamic interactions.

\subsection{Introduction}

Phase plane analysis has recently offered a unique perspective into cardiac fibrillatory behavior [1-5]. An excitable element may be mapped into phase space by plotting a state variable against another variable; for example, the two-variable FitzHugh-Nagumo model allows for phase space to be examined in terms of excitability versus refractoriness [6]. Normally, an excitable element remains at one stable location in phase space until a suprathreshold stimulus perturbs the system and forces the element into a closed-loop trajectory about an attractor.

For practical purposes, an experimenter may not have direct access to multiple, concurrent state variables in order to perform a dynamical systems analysis. However, using time-series analysis, a topologically equivalent attractor may be reconstructed given only one component of the system $[7,8]$. For $N$ evenly sampled values of $y(t)$, the attractor in two dimensions is $y_{i}=\left[\begin{array}{ll}y(i) & y(i+\tau)\end{array}\right]$ where $\tau$ is the time-embedding lag and $i=1, \ldots, N-1$.

Given a spatiotemporal activation sequence, we can use time-series analysis to reconstruct a phase space trajectory by using the transmembrane potential, $V(t)$, as a state variable, and plotting it against $V(t+\tau)$. Typically, $\tau$ is usually calculated as the first zero-crossing of the autocorrelation function for the data in order to insure linear independence between the two state variables [9]. We may then define phase, $\theta$, as the angle made by the phase space trajectory with respect to a central origin at a particular instant in time [1]. 


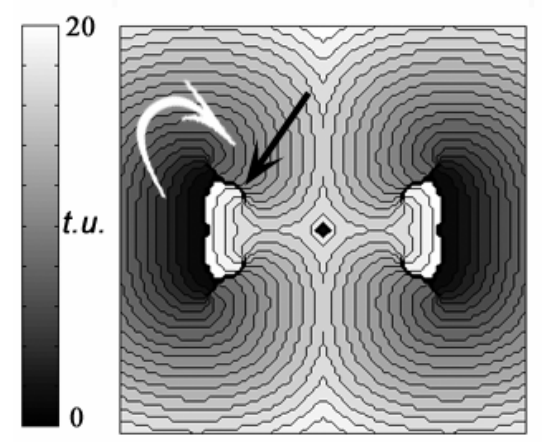

A

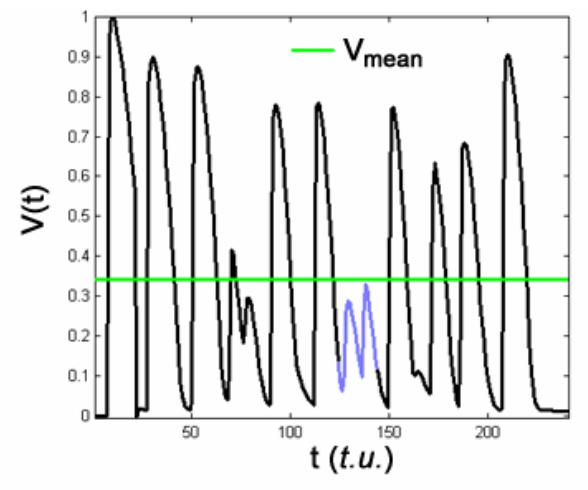

B

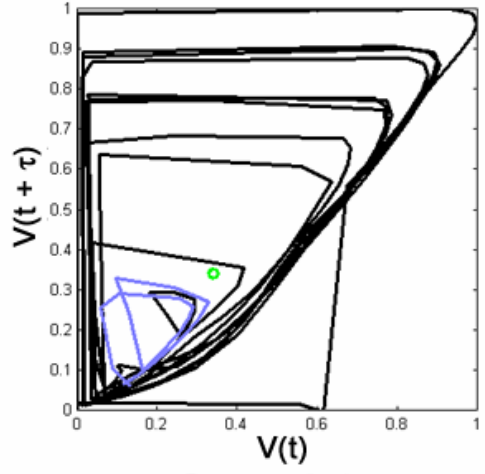

C: $\tau=3$ t.u.

Figure 4.1: (A) An isochronal map from numerically simulated data. The white arrow indicates direction of wave rotation. (B) Transmembrane signal measured at site indicated by the black arrow in (A). Oscillations falling below the mean value, $V_{\text {mean }}$, of the signal are drawn in blue. (C) Phase portrait of the signal shown in (B) using $\tau=3$. The origin $\left(V_{\text {mean }}, V_{\text {mean }}\right)$ is indicated by a circle.
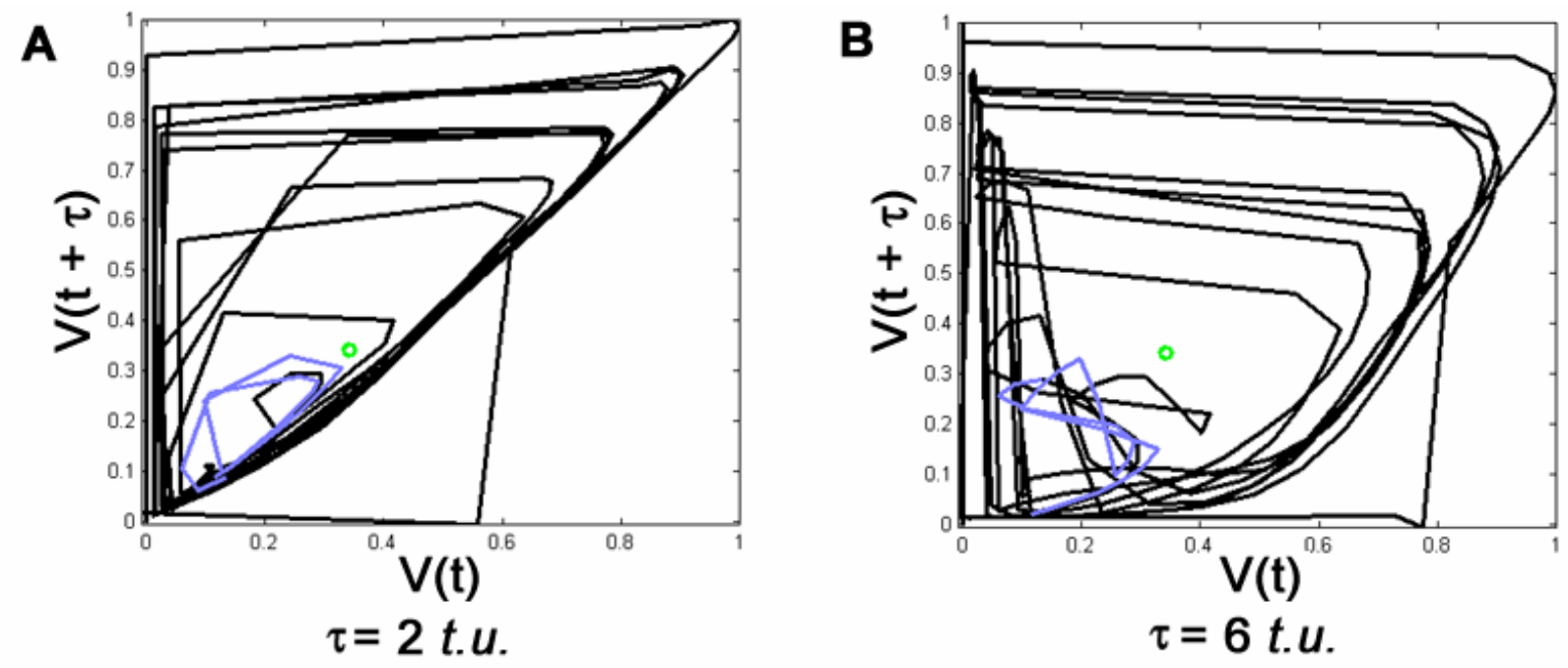

Figure 4.2: Example of phase space trajectories resulting from poor choices of $\tau$. (A) $\tau=3$. (B) $\tau$ $=6$.

Rotors are regions of excitability rotating around a central spatial location. In phase space, this point corresponds to the phase singularity, a topological defect where all phase values (i.e., $-\pi$ to $\pi$ ) converge and the phase itself at that point is undefined [10]. An example is shown in Figure 4.1A; here, an isochronal activation map is used to highlight the location of the rotor and the motion of the wave around it. Regions around the phase singularity are characterized by 
low amplitude oscillations $[11,12]$. In the phase plane, these correspond to rotations with a small circumferential length.

As described by Gray et al., [1] a fixed origin in the phase plane is defined by using the average value, $V_{\text {mean }}$, of the state variable $V(t)$ for the entire sequence about which the instantaneous phase angle is determined. However, in the case of unstable or drifting reentrant patterns such as fibrillation, low-amplitude passive responses may occur as the vortex meanders through or near a particular location. Therefore, one limitation of this approach for is that, for such a site, all cycles in the phase plane may not encircle the origin as defined by $V_{\text {mean }}$, as shown in Figure 4.1C. In addition, the selection of embedding delay $\tau$ is critical; a non-optimal choice of $\tau$ may lead to a low-quality reconstruction of phase space. If $\tau$ is too small, then $V(t) \approx V(t+\tau)$ (i.e., the values are highly correlated), resulting in the trajectories being concentrated on the diagonal in the reconstructed phase space, as shown in Fig. 4.2A. An overly large choice of $\tau$ leads to decorrelation of the data, resulting in stretching and deformation of the phase trajectories as the structure of the attractor is destroyed. If we define a proper rotation as one which has a definite direction and a unique center of rotation, we see from Fig. 4.2B, this choice of $\tau$ leads to several improper rotations along the trajectory, which will yield a distorted calculation of phase about $V_{\text {mean }}$. This in turn could lead to errors in interpreting the dynamics of phase singularities associated with reentry. Furthermore, during full fibrillation, the number of wavefronts and wave morphology changes unpredictably. Hence, the optimal value of $\tau$ as calculated by the autocorrelation method may not be unique for the entire spatiotemporal course of recorded activity. We propose an alternate method of reconstructing the phase space which takes into account the non-stationary nature of fibrillatory behavior and the pitfalls of a non-optimal choice of $\tau$.

\subsection{Methods}

The primary difficulty in calculating phase for this variety of oscillatory behavior is the presence of multiple centers of rotation in the phase plane during the temporal evolution of the system. A solution is to modify the state variable in an attempt to insure a fixed center of rotation [13]. This procedure is dependent on the proper implementation of the empirical mode decomposition (EMD) [14], which divides the signal into a series of intrinsic mode functions, each of which represents an oscillation frequency embedded within the signal. However, the 
process grants proper rotations to all deflections in the signal, regardless of amplitude. Therefore, oscillations stemming from noise are treated the same as oscillations generated from action potential propagation. A companion problem is the iterative nature of the procedure, which can result in inappropriate exaggeration of even the most minute deflections. Therefore, there is not necessarily a good correlation between an intrinsic mode of a temporal signal at a particular location and its neighbor. Hence, the EMD does not easily lend itself to analysis of a spatially distributed series. We have modified the algorithm in order to include considerations unique to cardiac data.

\subsubsection{The pseudo EMD (pEMD)}

Since not every oscillation in the data is significant, the first task is to determine which oscillations are given proper rotations in the phase plane. To this end, the algorithm first performs a pEMD upon the data. First, we construct two envelope curves which connect the maxima, $V_{\max }(t)$, and minima, $V_{\min }(t)$, of the wave form. This is performed by applying a sliding maximum and minimum filter to the data. The length of the filter window is determined in the following way. The period of the reentrant activity was estimated on the basis of the dominant frequency component of the data; since much of tissue is at some distance from the phase singularities present, it was assumed that the period obtained would be representative of single action potentials alone. Since reentry may be characterized by double-humped potentials $[15,16]$, we then used half this value as the filter window length in order to also isolate the double peaks. Applying these two filters and subtracting the results will yield zeros surrounding the extrema. The result can further be processed to produce the actual extrema points. As opposed to [14], we have chosen to use piecewise cubic Hermite rather than cubic spline interpolation to connect the extrema since cubic spline interpolation may create large swings between extrema. The envelope midline $V_{\text {mean }}{ }^{*}(t)$ is then computed as $\left[V_{\max }(t)+V_{\min }(t)\right] / 2$. We then detrend $V(t)$ by computing $V^{\prime}(t)=V(t)-V_{\text {mean }}{ }^{*}(t)$. In this way, we create a rough approximation of an intrinsic mode function with the pertinent frequency information desired. 


\subsubsection{The Hilbert transform}

Mathematically, a function and its Hilbert transform are orthogonal over the infinite interval. Hence plotting $V^{\prime}(t)$ against its Hilbert transform will yield proper rotations in the phase plane. The Hilbert transform is calculated as [17]

$$
H\left[V^{\prime}(t)\right]=\frac{1}{\pi} \mathrm{P} \cdot \mathrm{V} \cdot\left[\int_{-\infty}^{\infty} \frac{V^{\prime}(T)}{t-T} d T\right]
$$

where P.V. is the Cauchy principal value of the integral. For practical purposes, this integral is never actually calculated; it is obtained from $V^{\prime}(t)$ by a filter with a unity amplitude response and a phase response with a constant $\pi / 2$ lag at all frequencies. $V^{\prime}(t)$ and $H\left[V^{\prime}(t)\right]$ may be combined as complex conjugates to form an analytical signal,

$$
\tilde{V}(t)=V^{\prime}(t)+j \cdot H\left[V^{\prime}(t)\right]=A(t) e^{i \theta(t)}
$$

where $A(t)$ is an amplitude function, and $\theta(t)$ is a phase function.

The application of the pEMD and the Hilbert transform is displayed in Fig. 4.3 for a numerically simulated signal.

\subsubsection{Calculation of phase}

Using a constant $V_{\text {mean }}$, the spatial phase map for each coordinate $(x, y)$ is calculated as

$$
\theta(x, y, t)=\arctan \left(\frac{V(x, y, t+\tau)-V_{\text {mean }}(x, y)}{V(x, y, t)-V_{\text {mean }}(x, y)}\right)
$$

whereas the expression of phase using the Hilbert transform is

$$
\theta(x, y, t)=\arctan \left(\frac{V^{\prime}(x, y, t)}{H\left[V^{\prime}(x, y, t)\right]}\right)
$$

The formulation of $\theta$ in (4.4) is the same as what follows from the definition of the analytical signal given in (4.2). Also note that the new formulation is independent of any time-embedded delay, $\tau$.

\subsubsection{Numerical simulation and experimental preparation}

The numerical methods are identical to those described previously $[11,12,18]$. The tissue was modeled as a 3-D bidomain using the Beeler-Reuter model for the active membrane kinetics 

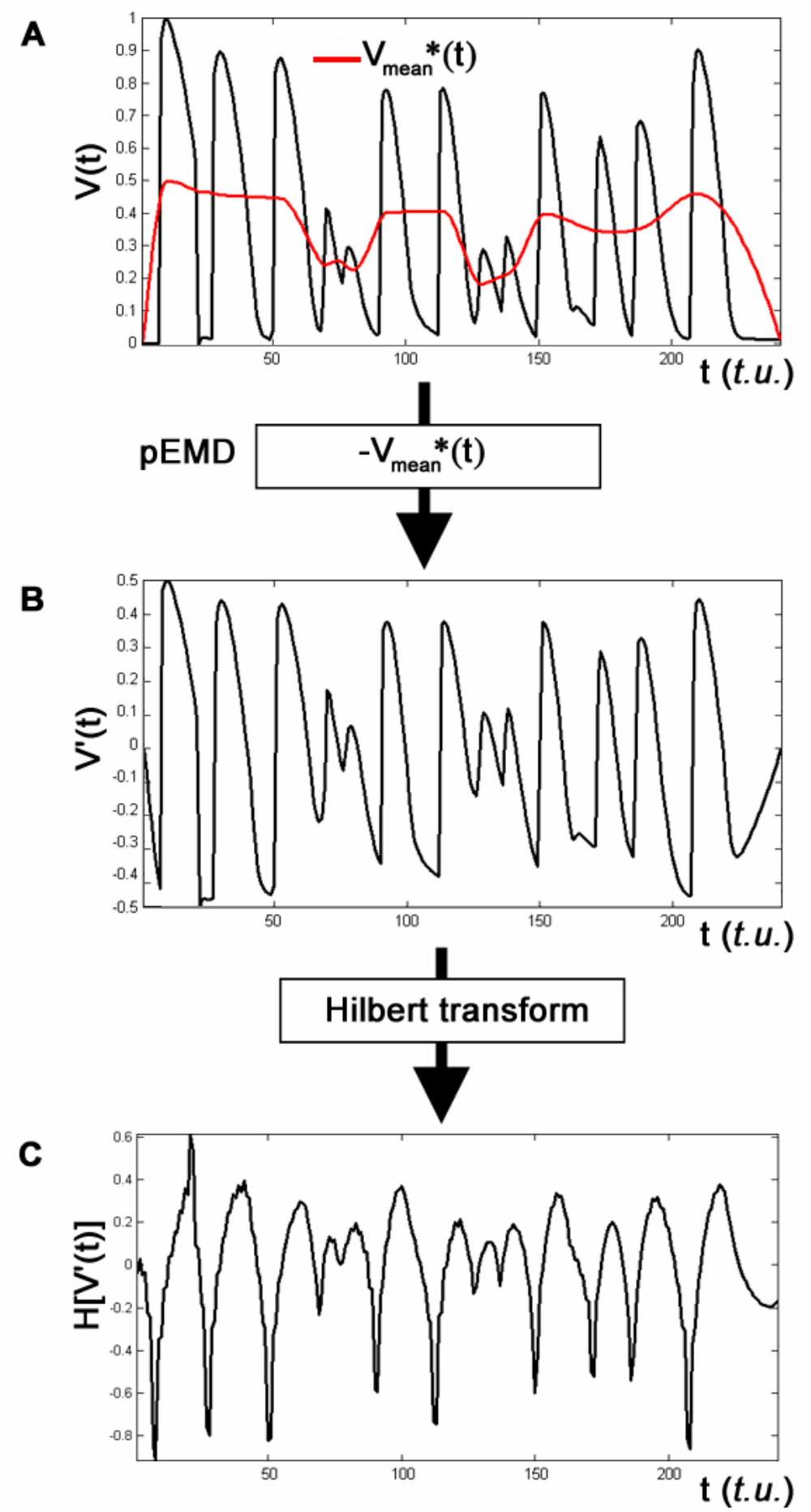

Figure 4.3: Flowchart of the algorithm as applied to numerically simulated data. (A) $V(t)$ with $V_{\text {mean }} *(t)$ shown as red line. (B) $V^{\prime}(t)$, the result of subtracting $V_{\text {mean }} *(t)$, as produced by the pEMD, from $V(t)$. (C) The Hilbert transform of $V^{\prime}(t)$. 


$$
\begin{aligned}
& \dot{V}=D \nabla^{2} V-\left(i_{N a}+i_{K 1}+i_{x 1}+i_{S}+i_{e x t}\right) / C, \\
& \dot{y}_{k}=f\left(y_{k}, V\right), h=2-8,
\end{aligned}
$$

where $D$ is the diffusion coefficient, $C$ is the membrane capacitance, $i_{N a}$ is a fast inward sodium current, $i_{K l}$ is time-independent outward potassium current, $i_{x l}$ is a time-activated outward current, $i_{S}$ is a slow inward calcium current, $i_{e x t}$ is the external injected current, and $y_{k}$ are a number of gating variables.

The experimental protocols, high-speed optical imaging system, and signal-processing methods have been described previously [12]. A conditioning stimulus (S1) through an electrode renders the tissue in a refractory state. A subsequent premature stimulus delivered through the same electrode $(\mathrm{S} 2)$, produces unidirectional conduction block such that quatrefoil reentry, a spiral wave pattern with fourfold symmetry, is created. The same stimulation protocol is used for the numerical simulations. For the experimental setup, spatiotemporal movies were recorded at 267 frames/sec in an image format of $96 \times 64$ pixels $(20.0 \times 13.5 \mathrm{~mm})$ for 300 frames. For the purposes of this manuscript, we express $\tau$ in time units $(t . u$. $)$ of image frames. Spatial and temporal filtering was applied to improve the signal-to-noise ratio. Both the experimental and the numerically simulated data were normalized to the range $[0,1]$ with the resting potential as 0 and the amplitude of the $\mathrm{S} 1$ stimulus as 1.

Singularity detection was performed using the algorithm described in [3]. In brief, the concept of topological charge is implemented as a series of convolution operations to detect a spatial phase distribution of $2 \pi$ around a pixel, the distinguishing characteristic of a phase singularity. These points are assigned a topological charge of \pm 1 , depending on chirality; elsewhere, the pixels are assigned a value of zero.

\subsection{Results}

The pEMD-Hilbert transformed phase trajectory corresponding to Figure $4.1 \mathrm{C}$ is shown in Figure 4.4A, where it can be seen that each orbit corresponds to a single proper rotation about the origin $(0,0)$. Fig. 4.4B is an expanded view of Fig. 4.4A, showing a portion of the trajectory with the same segment as in Fig. 4.1B highlighted with a broken line. We see that the origin is encircled by both these loops.

The difference in the phase maps can be seen in Figure 4.5. Figure 4.5A shows the phase map using a fixed center of rotation and $\tau=3$, whereas Fig. 4.5B is generated using the pEMD- 
Hilbert transformed data. Fig. 4.5C shows a difference map, the result of subtracting the first two panels from each other. Within the phase map, the phase singularities may be identified as those areas where all the colors converge to a single point. The most significant differences from the time-series method are concentrated around the vicinity of the phase singularities, which is expected since it is those regions where $V_{\text {mean }}$ is most likely to miss rotations in the phase plane, while yielding relatively unchanged values at larger distances from the singularities.

The dependence of the localization of the phase singularity on the choice of $\tau$ is shown in Fig. 4.6. Of note is the disparity in the location of the singularities using a constant center of rotation, especially in Fig. 4.6C, where extraneous singularities are visible. The singularities created with the pEMD-Hilbert transform bear the most resemblance to those generated using $\tau=$ 3 ; while $\tau=7$ was calculated by the autocorrelation function to be the optimal value for the embedded delay, lowering $\tau$ to 3 actually provided the least amount of trajectory deformation in the vicinity of the singularities.

We examined the difference between the locations of the singularities as defined using a fixed center of rotation and a $\tau$ of 3 , and using the pEMD-Hilbert transform. The average difference was $2.3 \pm 1.9$ spatial units (on a grid of $101 \times 101$ units). We also observed that the difference tended to be the greatest when the spiral was experiencing its greatest degree of meander.

Applying this formulation to experimental data (an isochronal activation map is shown in Fig. 4.7A) yields similar results. Figure 4.7B displays an unprocessed sample waveform taken from a data set exhibiting quatrefoil reentry. The dotted regions in Fig. 4.7B highlight a region where a phase singularity is present. The corresponding phase trajectory is shown in Fig. 4.7 C, which exhibits some improper rotations. Again, we calculate $V_{\text {mean }}{ }^{*}$ for this waveform, generate $V^{\prime}(t)$ and subsequently $H\left[V^{\prime}(t)\right]$, as seen in Figure 4.8. The corresponding pEMD-Hilbert transformed phase trajectory to Fig. 4.7A is shown in Figure 4.9A, where it can be seen that each orbit corresponds to a single proper rotation about the origin $(0,0)$. Figure $4.9 \mathrm{~B}$ is an expanded view of Fig. 4.9A, showing a portion of the trajectory with the same segment as in Fig. 4.7A highlighted with a broken line. Like the numerically simulated waveform, we see that the origin is encircled by these loops.

The difference in the phase maps can be seen in Figure 4.10. Figure 4.10A shows the phase map using a fixed center of rotation and $\tau=7$, whereas Fig. 4.10B is generated using the 

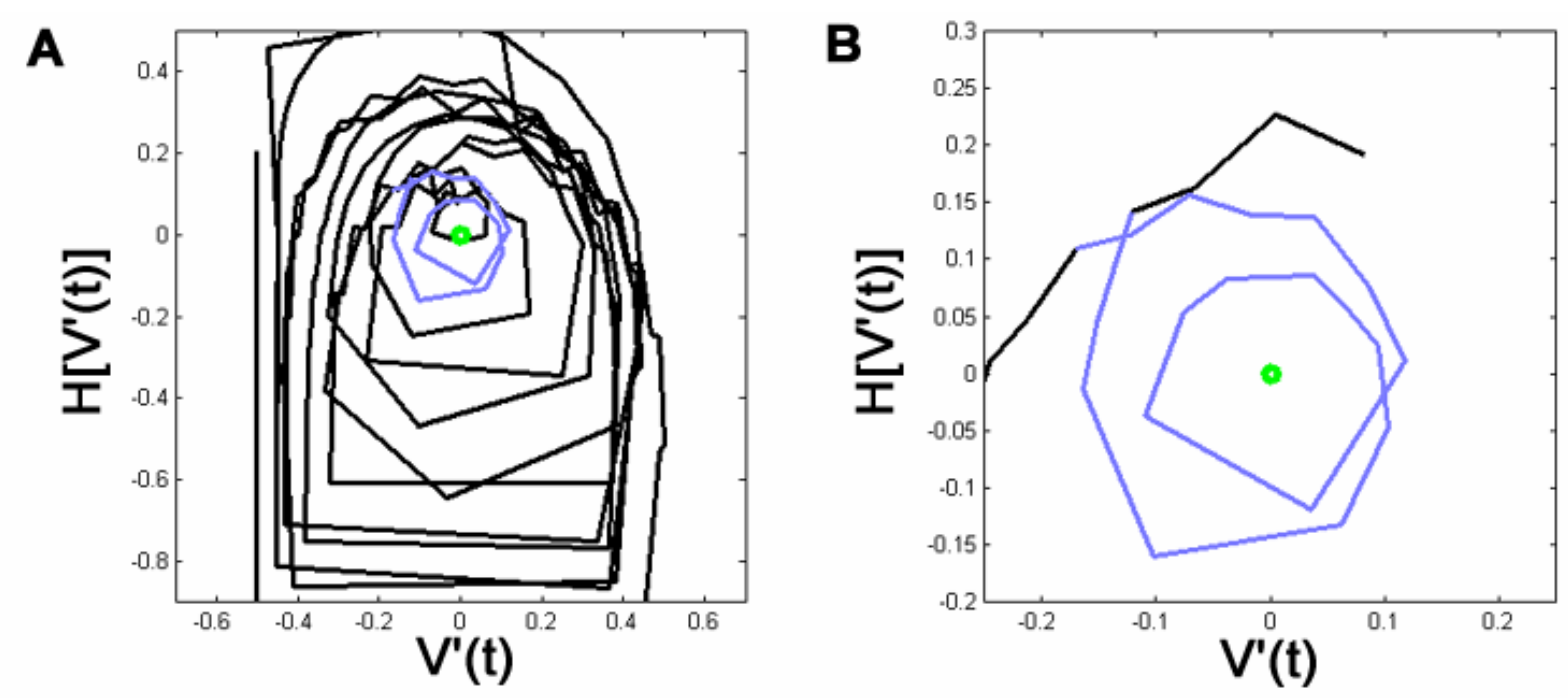

Figure 4.4: (A) Example of phase trajectory using Hilbert transform. Circle indicates origin $(0,0)$, same segment from Fig. 4.1B drawn in blue. (B) Expanded view of (A) illustrating the highlighted segment.
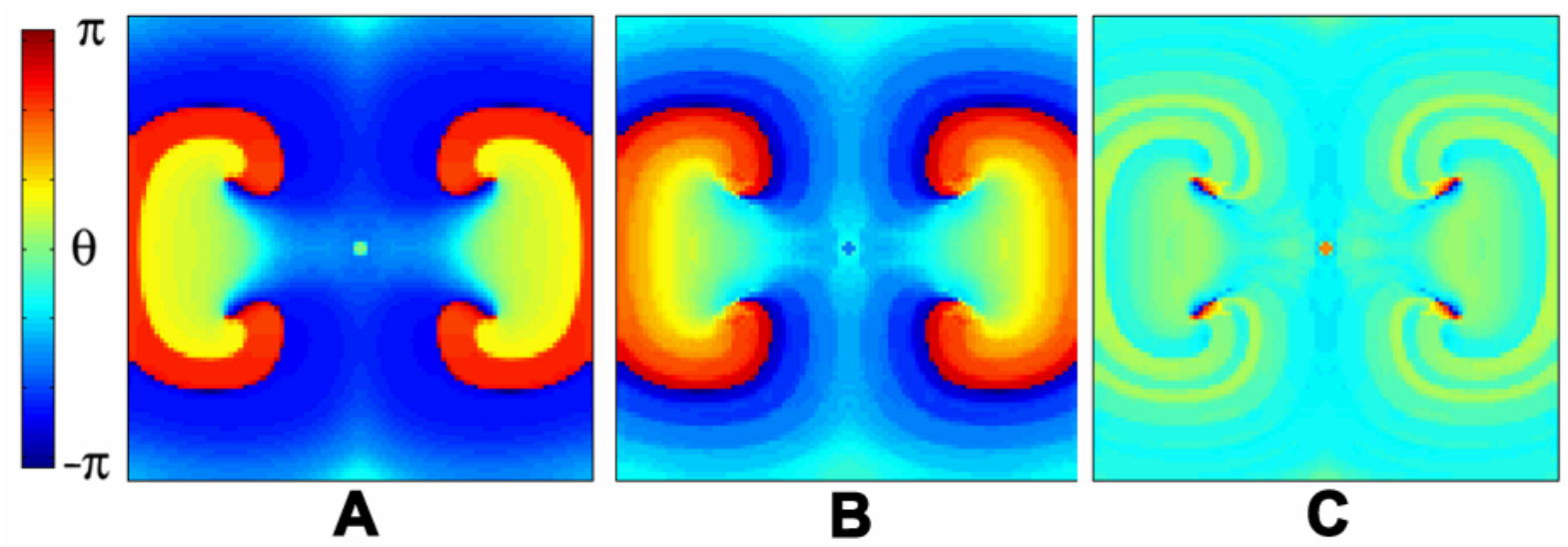

Figure 4.5: Comparison of phase maps calculated from numerically simulated data. (A) Map calculated using $\tau=3$. (B) Map calculated using Hilbert transform. (C) Difference map created by subtracting $(\mathrm{A})$ and $(\mathrm{B})$. 


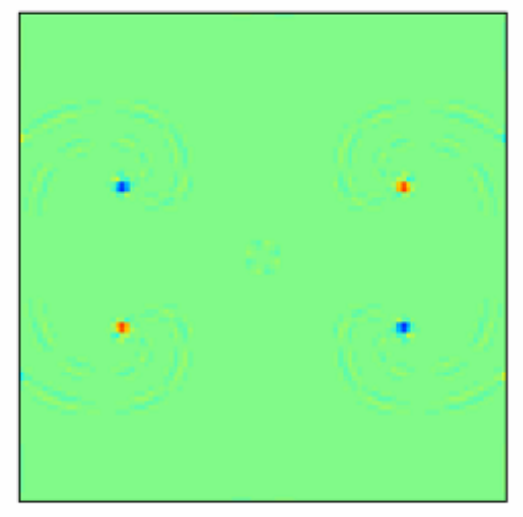

A: $\tau=3$

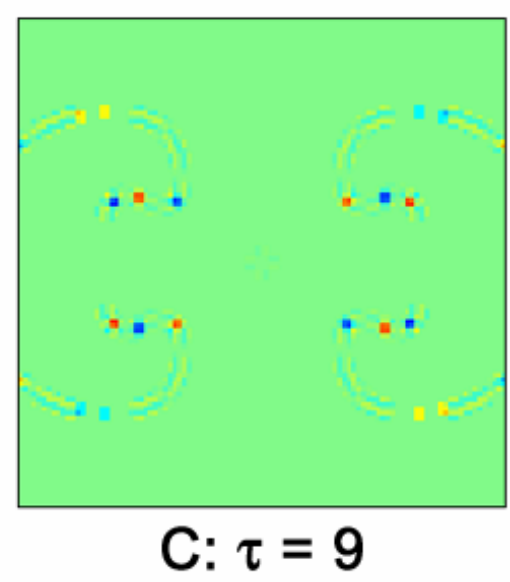

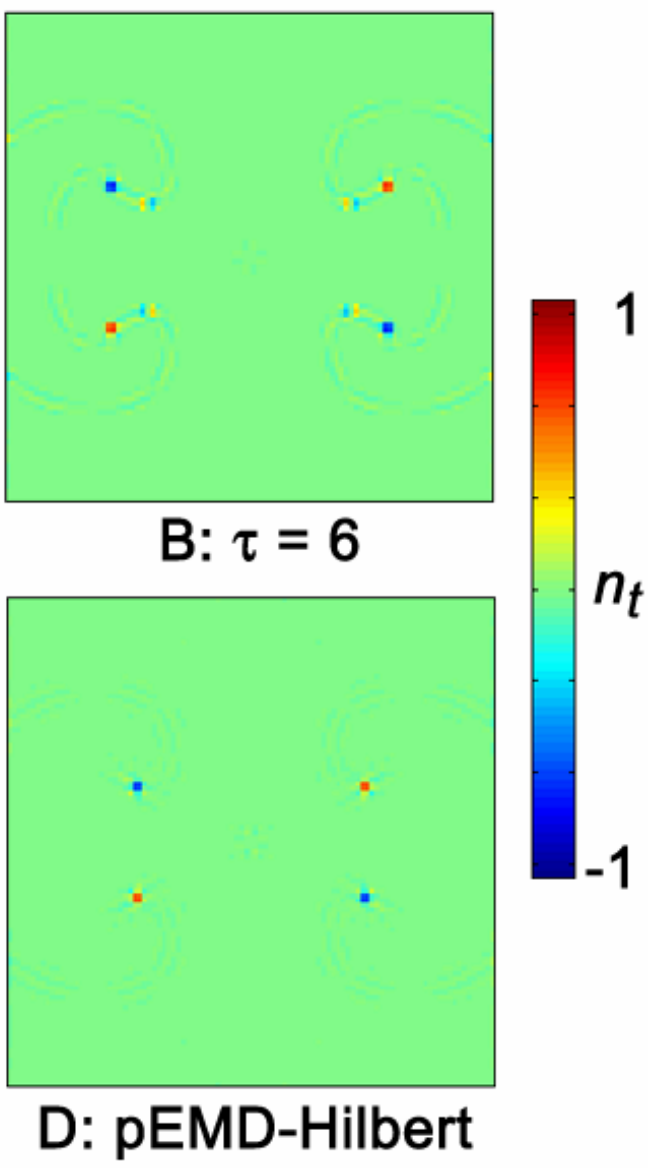

D: pEMD-Hilbert

Figure 4.6: Comparison of phase singularity maps generated from various values of $\tau$ and the pEMD-Hilbert transform. (A) $\tau=3$; (B) $\tau=6$; (C) $\tau=9$; (D) pEMD-Hilbert transform.

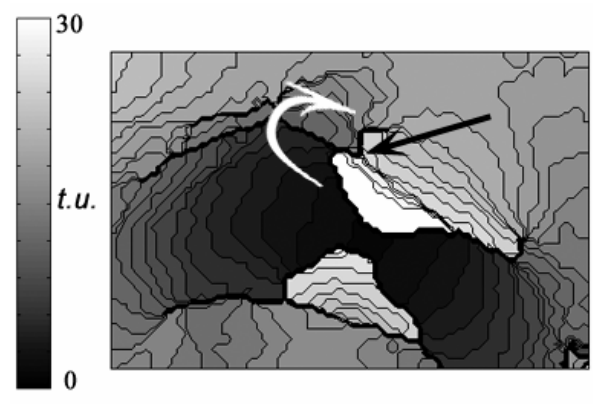

A

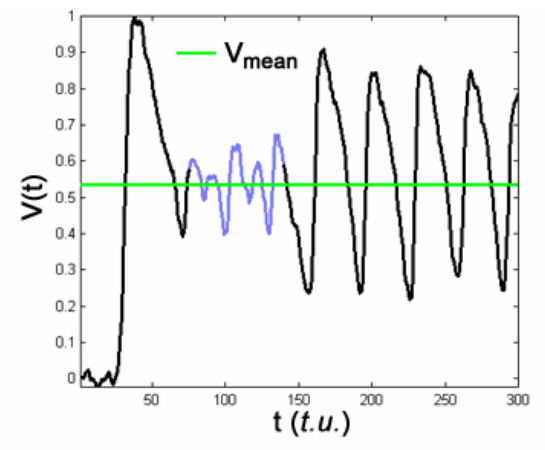

B

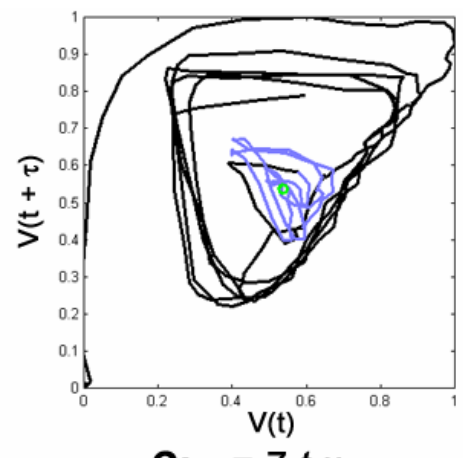

C: $\tau=7$ t.u.

Figure 4.7: (A) An isochronal map from experimental data. The white arrow indicates direction of wave rotation. (B) Transmembrane signal measured at site indicated by arrow in (A).

Oscillations indicating presence of the phase singularity shown in blue. (C) Phase portrait of the signal shown in (B) using $\tau=7$. Origin $\left(V_{\text {mean }}, V_{\text {mean }}\right)$ indicated by circle. 

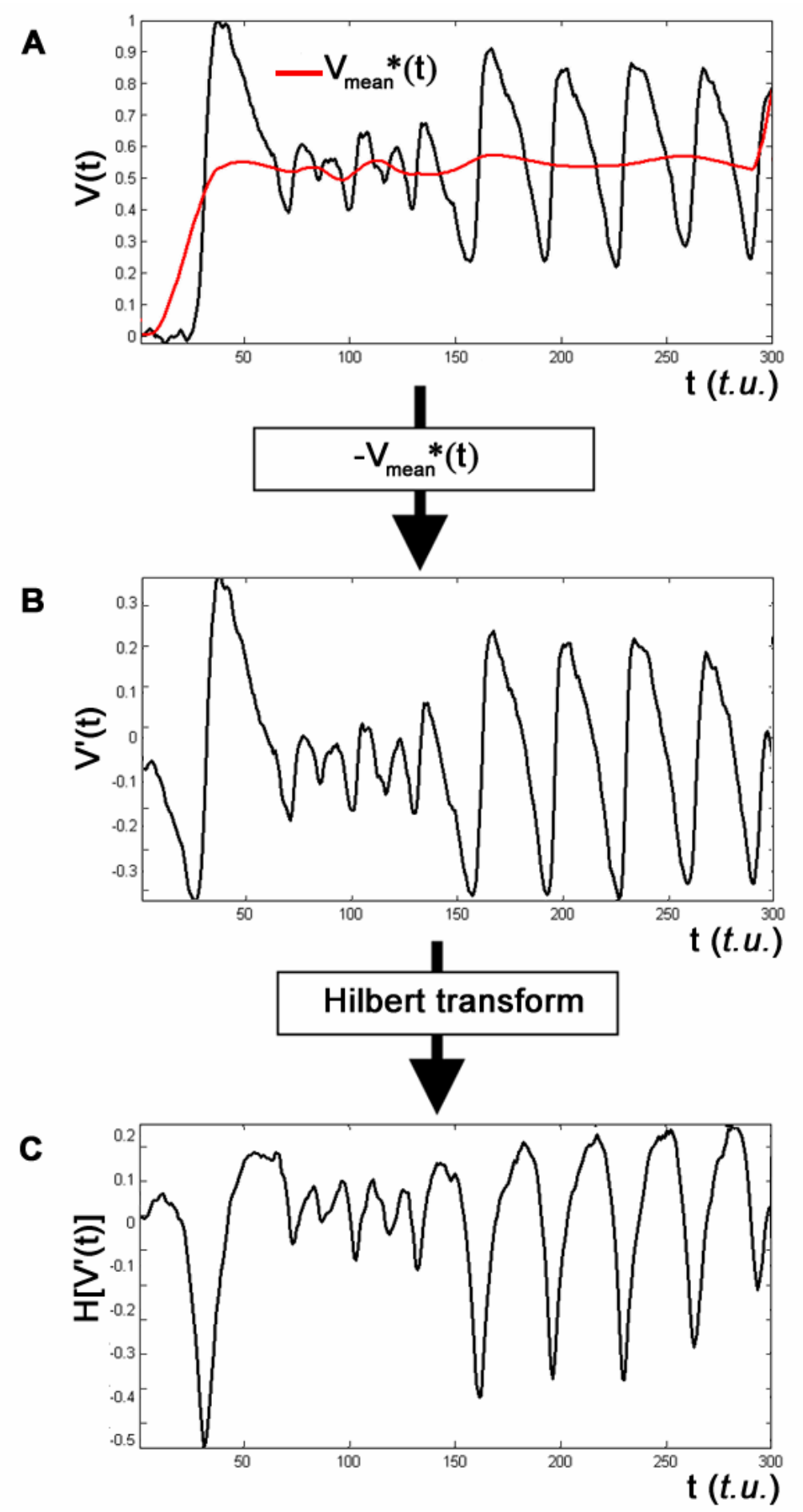

Figure 4.8: Flowchart of the algorithm as applied to experimental data. (A) $V(t)$ with $V_{\text {mean }} *(t)$ shown as red line. (B) $V^{\prime}(t)$, the result of subtracting $V_{\text {mean }} *(t)$, as produced by the pEMD, from $V(t)$. (C) The Hilbert transform of $V^{\prime}(t)$. 

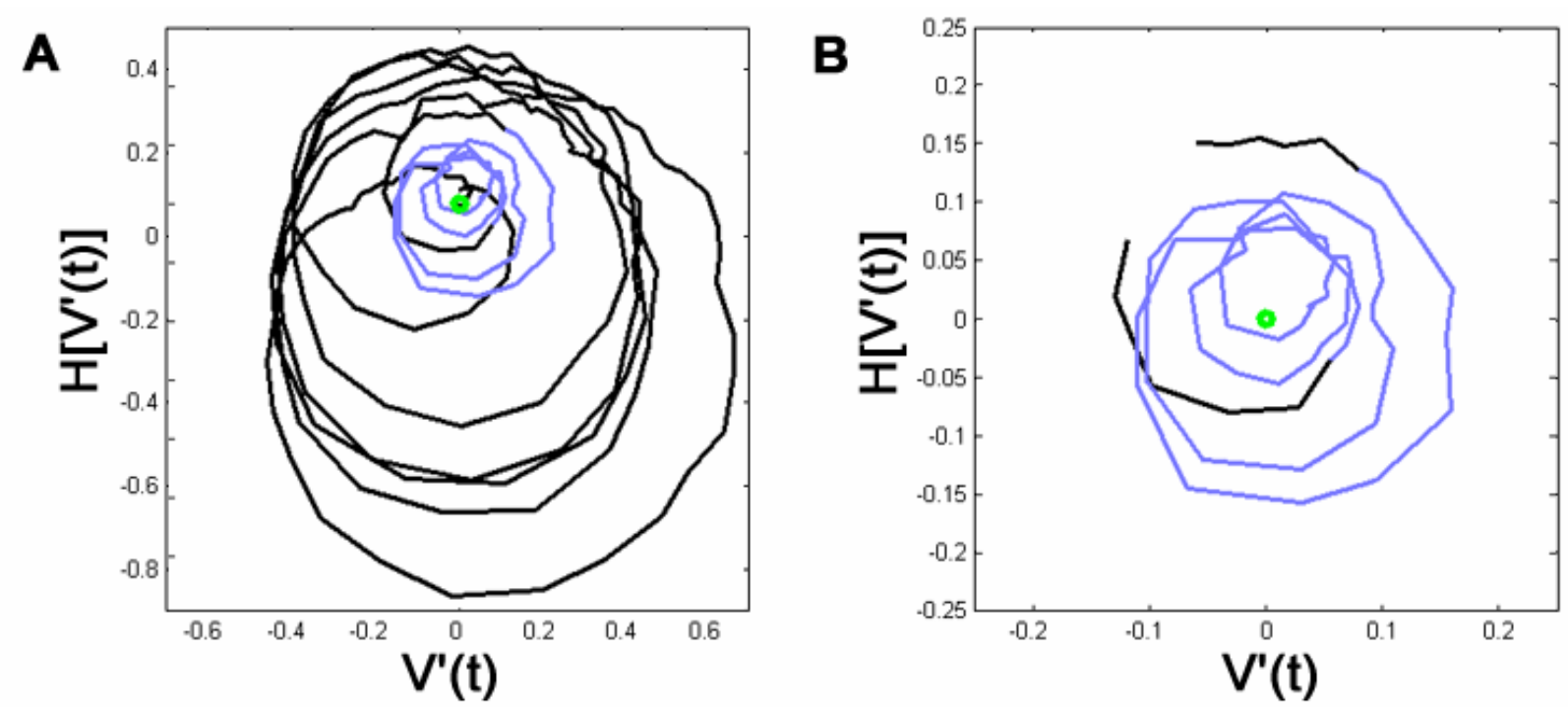

Figure 4.9: (A) Example of phase trajectory using pEMD-Hilbert transform. The circle indicates the origin $(0,0)$, the same segment from Fig. 4.7B is shown in blue. (B) Expanded view of (A) illustrating the highlighted segment.

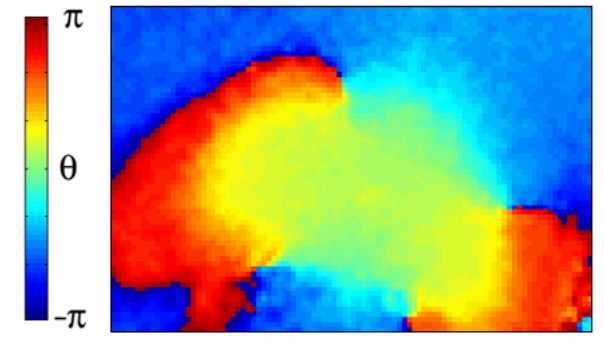

A

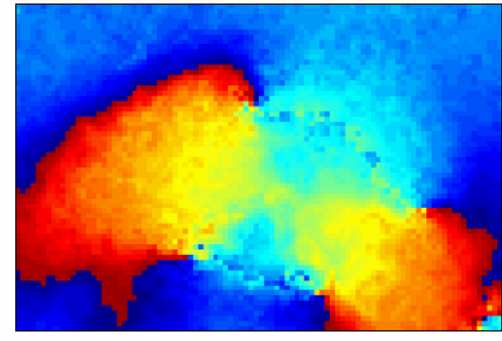

B

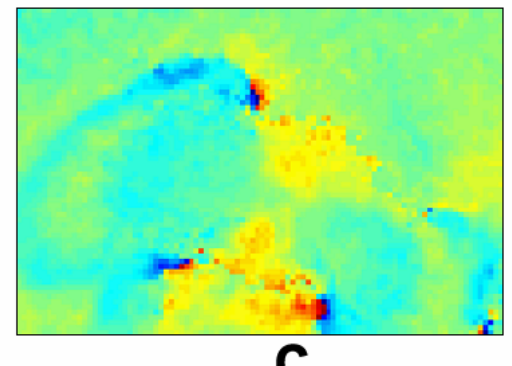

C

Figure 4.10: Comparison of phase maps calculated from the experimental data set used to obtain Figs. 4.7-4.9. (A) Map calculated using $\tau=7$. (B) Map calculated using the pEMD-Hilbert transform. (C) Difference map created by subtracting (A) and (B). 


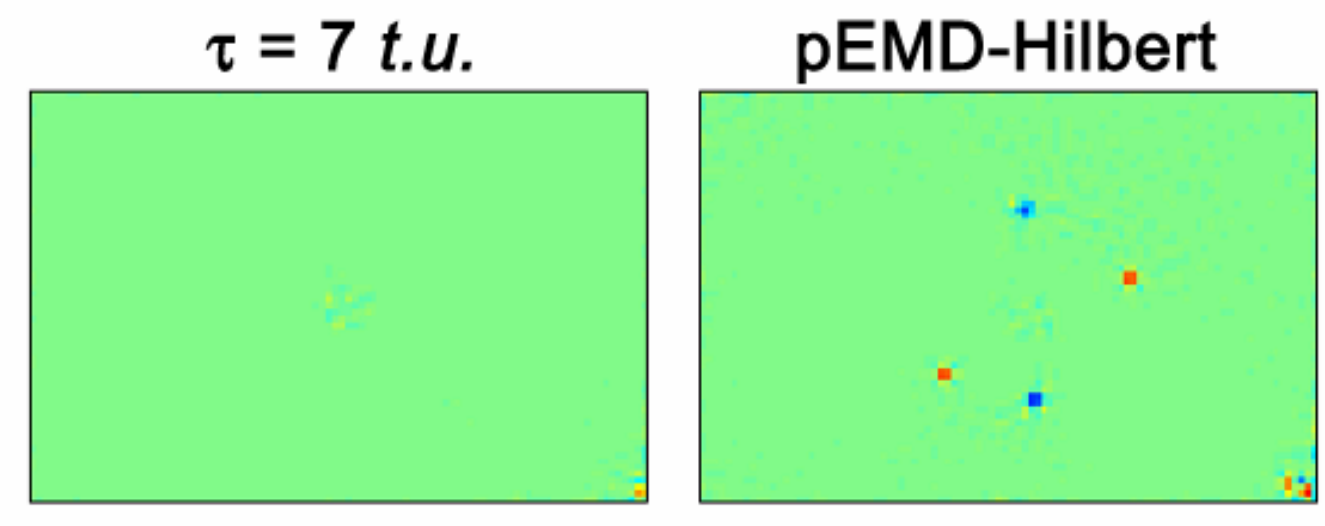

A: $\quad \mathrm{S} 2+3$ t.u.

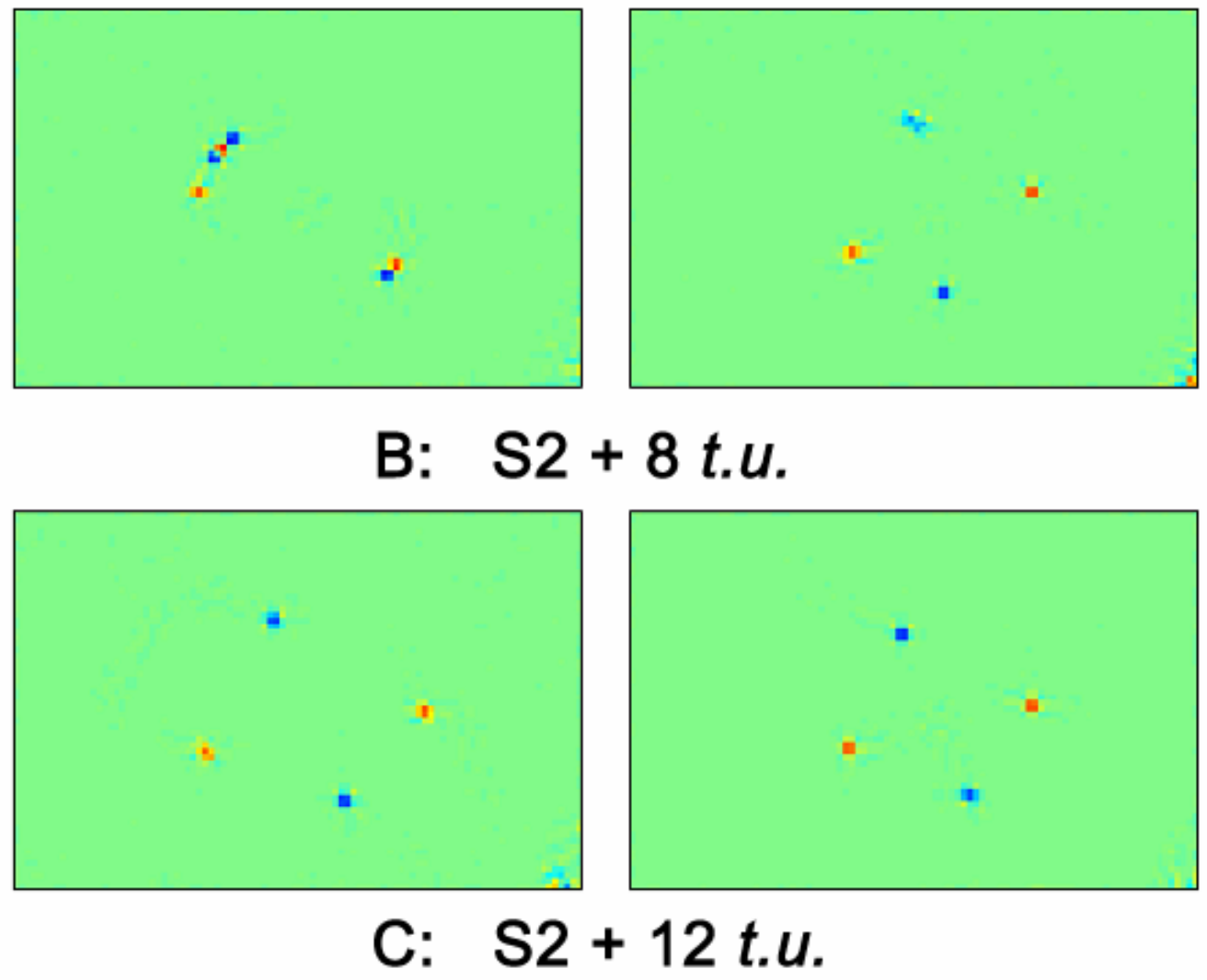

Figure 4.11: Phase singularity maps during S2 stimulation using experimental data using $\tau=7$ (left column) and the pEMD-Hilbert transform (right column). S2 + (A) 3, (B) 8, and (C) 12 t.u.. 

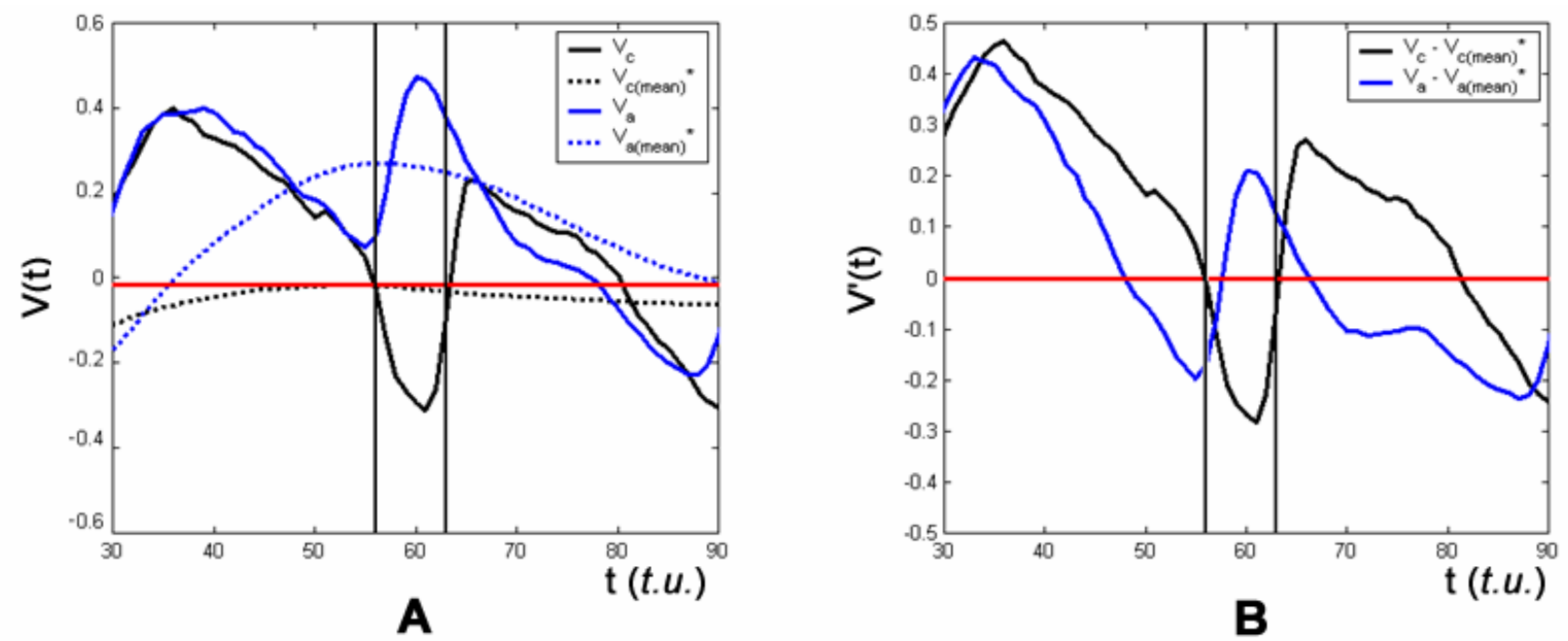

Figure 4.12: (A) V(t) traces from point located within virtual cathode (solid black curve) and virtual anode (solid blue curve); corresponding $\mathrm{Va}(\mathrm{mean}) *(\mathrm{t})$ and $\mathrm{Vc}($ mean $) *(\mathrm{t})$ traces for each measurement point (dotted lines); Vmean defined as zero (broken line). Vertical lines show duration of S2 stimulus. (B) $\mathrm{V}^{\prime}(\mathrm{t})$ traces corresponding to $\mathrm{V}(\mathrm{t})$ in $(\mathrm{A})$. Zero line drawn in red.
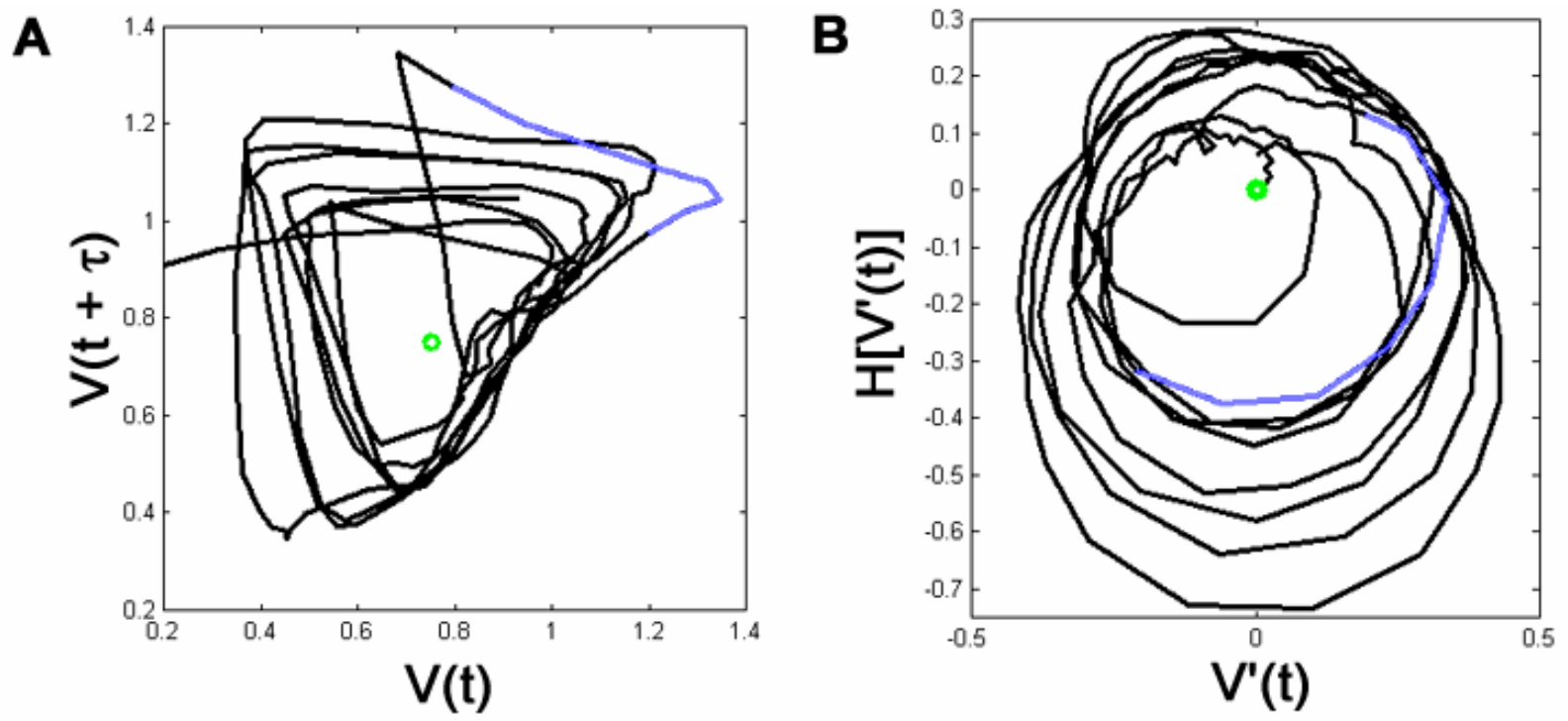

Figure 4.13: Phase trajectories for the data at the virtual cathode in Fig. 4.12 with the segment corresponding to the duration of S2 stimulus drawn in blue. (A) $=7,(B)$ pEMD-Hilbert transform. 
pEMD-Hilbert transformed data. Fig. 4.10C shows the difference map; once again, the regions with highest difference magnitude are located at the singularities, although some noise can be seen in Fig. 4.10B. The average difference in singularity localization between the two methodologies was $0.52 \pm 0.47 \mathrm{~mm}$ (in a field of view of $20.0 \times 13.5 \mathrm{~mm}$ ).

An additional consequence of using $V_{\text {mean }}{ }^{*}$ is that we are able to observe the initial phase singularity formation much closer to the start of the $\mathrm{S} 2$ stimulus than is permissible with $V_{\text {mean }}$, as shown in Figure 4.11. Figure 4.11A is taken at a point 3 t.u. after the beginning of the S2 (S2 terminates after 7 t.u.); the singularities are not visible in the frame generated from $V_{\text {mean }}$ because the improper rotations in the phase plane during S2 result in a miscalculation of phase and therefore, a disruption in singularity localization. This finding is important for the purpose of automated phase singularity tracking and for examination of the early dynamics of the phase singularity.

\subsection{Discussion}

Computation of the spatial phase map is important for tracking phase singularity formation and behavior during fibrillatory activity. The standard means of calculating the phase map assumes a constant, fixed center of rotation in the phase plane, which may lead to missed or distorted rotations in the phase trajectory. Detrending the state variable on the basis of oscillation magnitude serves to create a non-stationary origin which takes the temporal evolution of the signal into account. The analytic signal of this new state variable generates a proper rotation in the phase plane (a $2 \pi$ rotation about the origin) while removing the dependence on the choice of time-embedded delay $\tau$. We have observed small differences in singularity localization depending on which methodology is used; while a discussion of absolute localization accuracy is difficult due to the lack of a "gold standard" of singularity identification, the pEMD-Hilbert transform algorithm attempts to correct for several recognized sources of error. In addition, this method has the effect of enabling phase singularity observation closer to initial formation than previously allowed.

\subsubsection{Observation of Initial Singularity Formation}

Virtual electrodes are critical to phase singularity formation and initial dynamics, and therefore their early detection is important $[3,19]$. The fact that phase singularities are visible 
earlier using the pEMD-Hilbert transform algorithm than the constant $V_{\text {mean }}$ methodology is evident from Figure 4.11; reducing $\tau$ to 3 causes the singularities to appear earlier but still not as early as the those produced by the pEMD-Hilbert transform algorithm The question remains as to whether these "early" singularities are indeed real or an artifact of the algorithm. In Fig. 4.12A, the optical signal during S2 from a virtual cathode $\left(V_{c}\right)$ is shown alongside a signal from the virtual anode $\left(V_{a}\right)$. The constant values of $V_{\text {mean }}$ for $V_{c}$ and $V_{a}$ has been subtracted from both curves such that $V_{\text {mean }}$ from this point on is now zero for both curves (shown as a broken line). We see that while the zero-line origin intersects the hyperpolarization trough at $V_{a}$, it completely misses the depolarizing peak at $V_{c}$. In terms of the phase plane, the depolarization from the virtual cathode advances the phase of this point and initiates reentry by producing a new cycle (type 0 or even phase resetting) [10]. Hence, the fact that this shift in phase is not captured as a full rotation around the origin in the phase plane is problematic. The appearance of the phase singularity is delayed until a neighborhood of pixels repolarize such that they create a $2 \pi$ distribution about the origin in the phase plane (recall that singularity detection requires a $2 \pi$ distribution of phase around a spatial point).

On the other hand, we see that both the anodal and cathodal traces are bisected by $V_{a(\text { mean })}{ }^{*}$ and $V_{c(\text { mean })}{ }^{*}$, respectively. Once $V^{\prime}(\mathrm{t})$ is generated by subtracting these midline traces, both $V_{c}$ and $V_{a}$ are distributed about the zero-line origin, as shown in Fig. 4.12B. Figure 4.13 illustrates $V_{c}$ from Fig. $4.12 \mathrm{~A}$ in the phase plane created using $V_{\text {mean }}$ where the $\mathrm{S} 2$ stimulus generates an improper rotation, whereas the use of $V_{\text {mean }}{ }^{*}$ brings the $\mathrm{S} 2$ oscillation into a proper rotation. The expected even phase resetting at $V_{c}$ is clearly seen which, along with $V_{c}$, produces an accompanying $2 \pi$ distribution of phase around the origin and the appearance of the corresponding singularity at the junction between the virtual electrodes.

\subsubsection{Limitations}

While this method generates proper rotations for selected oscillations in the waveforms, the question still remains of what is the smallest oscillation magnitude which should be allowed a proper rotation. For an example, note the small hump at $t=164$ t.u. in the top panel of Fig. 4.3

which is missed by $V_{\text {mean }}{ }^{*}$. The question is whether omitting this peak is acceptable or not. If it is part of a sub-threshold response, then it does not matter; unless the phase is reset somewhere in its vicinity, even though the phase value may be calculated differently, a phase singularity will 
not be detected. If instead it is a low amplitude response due to its proximity to the reentrant core, shifting its value such that it encircles the origin will cause a phase distortion which may impair singularity localization. In the case of the numerical data of Fig. 4.3, such a situation causes a slight smearing of the singularity. For experimental data, it is a larger concern since spurious extrema may create unwanted deflections in $V_{\text {mean }}{ }^{*}$. The magnitude of this issue is dependent on the window size used for the calculation of $V_{\text {mean }}{ }^{*}$, therefore, a compromise must be made between making the window so short it catches unwanted extrema, or so long that it compromises the selection of the double-peaked potentials. This issue is problematic because an excursion in $V_{\text {mean }}{ }^{*}$ is not transient but instead occurs over a duration lasting the length of time from the previous extrema to the subsequent extrema. Hence, phase values can be distorted over a wide length of time.

As mentioned above, it is worth noting that a small value of $\tau$ produces results similar to the pEMD-Hilbert algorithm, as compared to the larger value determined by the auto-correlation function (Fig. 4.7). For time-delay embedding method, choosing a value of $\tau$ close to the duration of the action potential upstroke tends to minimize the trajectory distortion in the phase portrait, especially in the case of fibrillation where the upstroke duration is prolonged [2]. Therefore, globally choosing a short $\tau$ for the time-delay embedding method achieves the same desired effect in the phase plane as the orthogonality of the Hilbert-transformed signal.

The algorithm presented still possesses a time-dependent component, the calculation of the pEMD; the effectiveness of the Hilbert transform is dependent on the proper determination of the pEMD. The window length is currently chosen not on the basis of the double oscillations themselves but inferred on the basis of the full action potentials. Using half the period of the full potential seems to be successful in most cases tested but this assumption may not apply to every set of cardiac waveforms. Setting the filter window too large may cause small oscillations situated between larger ones to be omitted entirely; setting the window too small runs the risk of incorrectly capturing deflections due to noise. Based on our experience, we have found it more desirable to err on the side of a shorter window when selecting the optimal window length. A more rigorous criterion for the calculating the pEMD is a subject for future research. 


\subsection{Acknowledgments}

This study was supported in part by NIH Grants P01HL46681, R01HL58241, and T32HL07411, a graduate research fellowship from the National Science Foundation, and a Biomedical Engineering Research Grant from the Whitaker Foundation. We thank Richard Gray for his comments and suggestions.

\subsection{References}

[1] Gray, R. A., Pertsov, A. M., and Jalife, J., "Spatial and temporal organization during cardiac fibrillation." Nature, vol. 392, no. 6671, pp. 75-78, 1998.

[2] Gray, R. A. and Jalife, J., "Video imaging of atrial defibrillation.," in Zipes, D. P. and Jalife, J. (eds.) Cardiac Electrophysiology: From Cell to Bedside 3rd ed. Philadelphia: W.B. Saunders, 1999, pp. 432-439.

[3] Bray, M.-A., Lin, S.-F., Aliev, R. R., Roth, B. J., and Wikswo, J. P., Jr., "Experimental and theoretical analysis of phase singularity dynamics in cardiac tissue." J Cardiovasc Electrophysiol, vol. 12, no. 6, pp. 716-722, 2001.

[4] Chen, J., Mandapati, R., Berenfeld, O., Skanes, A. C., and Jalife, J., "High-frequency periodic sources underlie ventricular fibrillation in the isolated rabbit heart." Circ Res, vol. 86, no. 1, pp. 86-93, 2000.

[5] Chen, J., Mandapati, R., Berenfeld, O., Skanes, A. C., Gray, R. A., and Jalife, J., "Dynamics of wavelets and their role in atrial fibrillation in the isolated sheep heart." Cardiovasc Res, vol. 48, no. 2, pp. 220-232, 2000.

[6] FitzHugh, R., "Impulses and physiological states in theoretical models of nerve membrane." Biophys J, vol. 1, no. 6, pp. 445-466, 1961.

[7] Packard, N. H., Crutchfield, J. P., Farmer, J. D., and Shaw, R. S., "Geometry from a time series." Phys Rev Lett, vol. 45, no. 9, pp. 712-715, 1980.

[8] Takens, F., "Detecting strange attractors in turbulence.," in Rand, D. A. and Young, L.-S. (eds.) Dynamical Systems and Turbulence Berlin: Springer-Verlag, 1981, pp. 366-381.

[9] Abarbanel, H. D. I., Brown, R., Sidorwich, J. J., and Tsimring, L. S., "The analysis of observed chaotic data in physical systems." Rev Mod Phys, vol. 65 pp. 1331-1392, 1993.

[10] Winfree, A. T., When Time Breaks Down: The Three-Dimensional Dynamics Of Electrochemical Waves And Cardiac Arrhythmias. Princeton: Princeton University Press, 1987. 
[11] Pertsov, A. M., Davidenko, J. M., Salomonsz, R., Baxter, W. T., and Jalife, J., "Spiral waves of excitation underlie reentrant activity in isolated cardiac muscle." Circ Res, vol. 72, no. 3, pp. 631-650, 1993.

[12] Lin, S.-F., Roth, B. J., and Wikswo, J. P., Jr., "Quatrefoil reentry in myocardium: An optical imaging study of the induction mechanism." J Cardiovasc Electrophysiol, vol. 10, no. 4, pp. 574-586, 1999.

[13] Yalcinkaya, T. and Lai, Y.-C., "Phase characterization of chaos." Phys Rev Lett, vol. 79, no. 20, pp. 3885-3888, 1997.

[14] Huang, W., Shen, Z., Huang, N. E., and Fung, Y. C., "Engineering analysis of biological variables: an example of blood pressure over 1 day." Proc Natl Acad Sci USA, vol. 95, no. 9, pp. 4816-4821, 1998.

[15] Allessie, M. A., Bonke, F. I. M., and Schopman, F. J. G., "Circus movement in rabbit atrial muscle as a mechanism of tachycardia: I." Circ Res, vol. 23 pp. 54-62, 1973.

[16] Olshansky, B., Moreira, D., and Waldo, A. L., "Characterization of double potentials during ventricular tachycardia. Studies during transient entrainment." Circulation, vol. 87, no. 2, pp. 373-381, 1993.

[17] Gabor, D., "Theory of communication." J IEE (London), vol. 93 (III), no. 26, pp. 429457, 1946.

[18] Roth, B. J., "Nonsustained reentry following successive stimulation of cardiac tissue through a unipolar electrode." J Cardiovasc Electrophysiol, vol. 8, no. 7, pp. 768-778, 1997.

[19] Wikswo, J. P., Jr., Lin, S.-F., and Abbas, R. A., "Virtual electrodes in cardiac tissue: A common mechanism for anodal and cathodal stimulation." Biophys $J$, vol. 69, no. 6, pp. 2195-2210, 1995. 


\title{
CHAPTER V
}

\section{Use Of Topological Charge To Determine Filament Location In A Numerical Model Of Scroll Wave Activity}

\author{
Mark-Anthony Bray ${ }^{1}$ and John P. Wikswo ${ }^{2}$ \\ ${ }^{1}$ Department of Biomedical Engineering \\ ${ }^{2}$ Department of Physics and Astronomy \\ Vanderbilt University
}

Nashville, TN 37235

Portions of this manuscript have been published in:

Bray, M-A and JP Wikswo, IEEE Transactions in Biomedical Engineering

Vol. 49, No. 10, pp. 1086-1093, October 2002.

(C) 2002 by IEEE. Reprinted with permission. 


\subsection{Abstract}

The unique time course of an excitable element in cardiac tissue can be represented as the phase of its trajectory in state space. A phase singularity is defined as a spatial point where the surrounding phase values changes by a total of $2 \pi$, thereby forming the organizing center for a reentrant excitatory wave, a phenomenon which occurs in cardiac fibrillation. In this paper, we describe a methodology to detect the singular filament in numeric simulations of threedimensional scroll waves by using the concept of topological charge. Here, we use simple twovariable models of cardiac activity to construct the state space, generate the phase field, and calculate the topological charge as a summation of 3-D convolution operations. We illustrate the usage of the algorithm on the basic dynamics of vortex ring filament behavior as well as the more complex spatiotemporal behavior observed in fibrillation. We also compare the motion of filament wavetips as determined by the phase field produced by two-variable state space and single-variable, time-delay embedded state space. Finally, we examine the state spaces produced by a more complex three-variable model. We conclude that the use of state space analysis, along with the unique properties of topological charge, allows for a novel means of filament localization.

\subsection{Introduction}

One of the more intriguing properties of excitable media driven by reaction-diffusion equations is the ability to support vortices, known as spiral waves in two dimensions and scroll waves in three dimensions [1]. The study of these phenomena is of vital importance in biology because such self-organizing systems seem to underlie ventricular fibrillation (VF) which leads to sudden cardiac death [2]. Spiral waves are characterized by rotation about a topological point defect known as a phase singularity. Since spiral waves are actually the two-dimensional crosssection of a scroll wave, the phase singularity may also be interpreted as the cross-section of three-dimensional line termed a filament.

While the motion of spiral waves can be observed experimentally in cardiac preparations [3-5], the direct observation of scroll waves and the accompanying filament is limited to twodimensional projections of three-dimensional chemical reactions [6,7]. In cardiac studies, the presence of scroll waves must be inferred from epicardial or endocardial recordings [8-11] or 
specialized transmural measurements $[12,13]$. For this reason, numerical models of excitable media are invaluable in providing information about the dynamic properties of filaments.

Previous studies have performed the localization of a numerically simulated filament in various ways. Another method detects the area for each spatial $z$ plane where the excitation remains below a minimal threshold over a single rotation period; the center of mass of these areas is taken to be the filament [14-17]. Another approach is to find the intersection of two successive isopotential surfaces (i.e., the line at which the isopotential temporal derivative is zero) $[18,19]$, which corresponds to detection of the wavebreak, a discontinuity in the wavefront such that the wavefront meets its own repolarizing tail. In a similar fashion, the intersection of the excitatory and recovery state variables for a particular value may also be used [14]. Other methods include finding the location of the maximum cross product of the state variable gradients [7] or the location of maximum curvature of a particular isopotential [20]. In this paper, we refine the methodology of localizing the filament using the concept of phase and topological charge $[3,21]$.

\subsection{Methods}

\subsubsection{Two-variable reaction-diffusion model}

To illustrate our method, we will first consider an excitable medium consisting of a two variable reaction-diffusion system described with the following partial differential equations

$$
\begin{aligned}
& \partial u / \partial t=D \nabla^{2} u+f(u, v), \\
& \partial v / \partial t=g(u, v),
\end{aligned}
$$

where $u$ is a fast variable analogous to the transmembrane potential, $v$ is a slow variable representing tissue recovery, $D$ is a diffusion coefficient in space units ${ }^{2} /$ time unit (in this case, we set $D=1)$, and $\nabla^{2}$ is the Laplacian operator.

\subsubsection{Two-variable state space and phase}

The term phase in cardiac literature indicates the unique state of an excitable element. The primary difficulty with using an activation value for the purposes of the singular point detection stems from the fact that the chosen value is encountered twice: once during depolarization and once during repolarization, and hence is not unique. However, by plotting $u(t)$ 
versus $v(t)$, we can represent the phase, $\phi(t)$, of each 3-D point of the model at $\vec{x}=(x, y, z)$ as an angular measurement with respect to an origin, $\left(u^{*}, v^{*}\right)$ as follows:

$$
\phi(\vec{x}, t)=\operatorname{atan}\left[v(\vec{x}, t)-v^{*}, u(\vec{x}, t)-u^{*}\right]
$$

where atan is the four-quadrant inverse tangent such that $\phi(\vec{x}, t)$ is in the range $[-\pi, \pi]$.

The choice of the origin in state space, $\left(u^{*}, v^{*}\right)$, is important to the calculation of phase and cannot be selected randomly. Ideally, the phase at a given spatial point should progress through $2 \pi$ during a complete rotation such that the phase is uniquely defined. This condition corresponds to this point generating a state space trajectory which encircles an origin during the course of the rotation. Because the amplitude of the action potential tends to decrease with increasing proximity to the filament, the trajectories from points close to the filament circumscribe smaller orbits. Hence, the ideal state space origin is one that is encircled by all trajectories regardless of the originating spatial location, though this point may vary depending on the excitable model used in the calculation. To choose this point, we performed a twodimensional spiral wave simulation using the chosen excitable model for a complete rotation after the spiral had been fully formed. Subsequently, the trajectories of all the points were plotted and the point in state space which was encircled by the smallest trajectories was selected as the origin.

\subsubsection{Time-series reconstructed state space}

In numerical simulations, the defined variables are available to the investigator for the purpose of constructing the state space. However, for practical purposes, an experimentalist may have access to only one observable variable at a time. However, the trajectories in twodimensional state space may be reconstructed from a single variable such that they are topologically equivalent to those in the multidimensional state space, by expanding the observed variable ( $u$, for instance) into a vector time series by embedding a time delay, $\tau$, into $u$ [22]. Taken's embedding theorem shows that for $N$ evenly sampled values of $u(t)$, the state space in two dimensions is

$$
u_{i}=[u(i), u(i+\tau)]
$$

where $\tau$ is the time-embedded delay and $i=1, \ldots, N-1$. It has previously been shown that the dynamics of cardiac activation behavior can be derived using time-delay embedding, or time- 
series analysis $[3,21]$, utilizing the transmembrane potential with a time-delay embedded version of the potential. Here, the phase is calculated as

$$
\phi(\vec{x}, t)=\operatorname{atan}\left[u(\vec{x}, t+\tau)-u^{*}, u(\vec{x}, t)-u^{*}\right]
$$

Note that the origin in this case is $\left(u^{*}, u^{*}\right)$.

The value of $\tau$ is chosen such that the phase can be uniquely specified during the course of a scroll wave rotation. Typically in nonlinear dynamics literature, an optimal choice of $\tau$ is one such that the mutual dependence of the two variables is minimized [23]. However, studies indicate that for cardiac activation, a $\tau$ that is on the order of the action potential upstroke duration reduces the amount of trajectory folding that would lead to a non-unique calculation of phase [24].

\subsubsection{Topological charge}

The phase singularity is the spatial point where all phase values converge and may be localized through calculation of the topological charge, $n_{t}$, defined as $[25,26]$

$$
n_{t} \equiv \frac{1}{2 \pi} \oint_{c} \nabla \phi \cdot d \vec{\ell}
$$

[26] where the line integral is taken over the path $\vec{\ell}$ on a closed curve $c$ surrounding the singularity; $n_{t}$ is an integer value whose sign depends upon the chirality of phase surrounding the singularity. By defining the integrand of Eq. (5.4), the gradient of the phase, as a wave vector field, $\vec{k}$, i.e, $\vec{k}(\vec{r}, t) \equiv \nabla \phi(\vec{r}, t)$, we see that by evaluating $n_{t}$ for a closed path in the limit as the area goes to zero in Eq. (5.4), the topological charge $n_{t}$ is proportional to the curl of the wave vector,

$$
\nabla \times \vec{k} \equiv\left(\frac{\partial k_{x}}{\partial y}-\frac{\partial k_{y}}{\partial z}\right) \hat{\mathrm{x}}+\left(\frac{\partial k_{x}}{\partial z}-\frac{\partial k_{z}}{\partial x}\right) \hat{\mathrm{y}}+\left(\frac{\partial k_{y}}{\partial x}-\frac{\partial k_{x}}{\partial y}\right) \hat{\mathrm{z}}
$$

Since $\vec{k}$ is derived from the gradient of a scalar field, the curl of $\vec{k}$ is zero everywhere where $\phi$ is differentiable, except at the phase singularity itself, where $\phi$ is undefined and there exists a net circulation of phase, as shown in Fig. 5.1.

\subsubsection{Computation of topological charge as a convolution operation}

For simplicity, we will show how the vector in (5.5) may be generated by examining the 

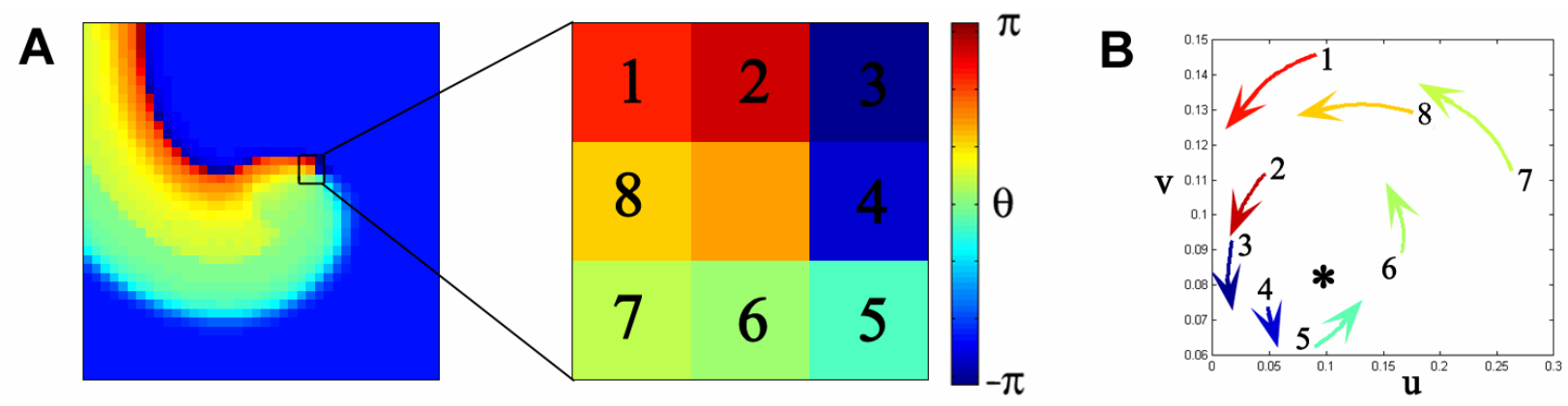

Figure 5.1: (A) A 2-D cross-section of a scroll wave mapped in the state space; inset shows the eight numbered pixels surrounding the singularity. The numbered ordering indicates the integration path and direction. (B) The position of the numbered pixels in the phase portrait; arrows show the trajectory of the pixels in the state space. The location of $\left(\mathrm{u}^{*}, \mathrm{v}^{*}\right)$ is shown with an asterisk.

$\hat{\mathbf{z}}$ component only. The $k_{z}$ component of $\vec{k}$ may be approximated from a discretized phase spatiotemporal sequence $\phi[m, n, p]$ with regular grid spacing by a centered, half-step finite difference operation in the $x$ and $y$ directions [27]

$$
\begin{aligned}
& k_{x}[m+1 / 2, n, p]=\nabla \phi_{x}[m+1 / 2, n, p]=\phi[m+1, n, p]-\phi[m, n, p], \\
& k_{y}[m, n+1 / 2, p]=\nabla \phi_{y}[m, n+1 / 2, p]=\phi[m, n+1, p]-\phi[m, n, p]
\end{aligned}
$$

According to Eq. (5.4), in order to localize a singularity, there must exist a $2 \pi n_{t}$ total change in phase around that point. Therefore, caution must be observed when calculating the gradient across the branch cut from $\pi$ to $-\pi$, such as between points 2 and 3 in the x-direction in Fig. 5.1. Otherwise, what is actually a continuous change in $v$ or $u$ between two adjacent pixels could be misrepresented as a discontinuity in phase, and hence incorrectly contribute to the total change in phase around that point. We accommodate for this by correcting any absolute phase jumps greater than $\pi$ in adjacent elements by converting them to their $2 \pi$ complement. Computation of the line integral in Eq. (5.4) at location $[m, n, p]$ can be expressed by the following convolution operation

$$
(\nabla \times \vec{k}) \cdot \hat{\mathrm{z}} \propto \nabla_{x}^{z} \otimes k_{y}+\nabla_{y}^{z} \otimes k_{x}
$$

where $\otimes$ is the convolution operator, and

$$
\nabla_{x}^{z}=\left[\begin{array}{cc}
+1 & -1 \\
0 & 0
\end{array}\right] \text {, and } \nabla_{y}^{z}=\left[\begin{array}{cc}
-1 & 0 \\
+1 & 0
\end{array}\right]
$$


are the $2 \times 2$ convolution kernels which we term Nabla kernels. The subscript indicates the axis parallel to the path segment, and the superscript indicates the plane to which the kernel is normal. The wave vector values lie between the grid nodes (Eq. (5.6)), but since our grid discretization cannot accommodate data at half intervals, they must be mapped to the grid nodes, i.e., $k_{x}[m+$ $1 / 2, n, p]$ and $k_{y}[m, n+1 / 2, p]$ are mapped to $k_{x}[m, n, p]$ and $k_{y}[m, n, p]$, respectively. Hence, the skewed appearance of the kernels reflect this configuration.

The other components of $\vec{k}$ may be calculated in a similar manner. Eq. (5.5) now becomes

$$
\nabla \times \vec{k} \propto\left(\nabla_{z}^{x} \otimes k_{y}+\nabla_{y}^{x} \otimes k_{z}\right) \hat{\mathrm{x}}+\left(\nabla_{x}^{y} \otimes k_{z}+\nabla_{z}^{y} \otimes k_{x}\right) \hat{\mathrm{y}}+\left(\nabla_{x}^{z} \otimes k_{y}+\nabla_{y}^{z} \otimes k_{x}\right) \hat{\mathrm{z}}
$$

The Nabla kernels are basically the same as those in Eq. (5.8), but aligned such that the path integral is evaluated along their respective normal planes. Hence, when implemented, the other Nabla kernels are rank-3 tensors; $\nabla_{y}^{x}$ and $\nabla_{z}^{x}$ are $2 \times 1 \times 2$ tensors such that

$$
\begin{aligned}
& \nabla_{y}^{x}=\left\{\nabla_{y}^{x^{(111)}}=-1, \nabla_{y}^{x^{(211)}}=+1,0 \text { elsewhere }\right\}, \\
& \nabla_{z}^{x}=\left\{\nabla_{z}^{x^{(111)}}=+1, \nabla_{z}^{x^{(112)}}=-1,0 \text { elsewhere }\right\},
\end{aligned}
$$

which perform the path integral within the $x$ plane, and $\nabla_{y}^{x}$ and $\nabla_{z}^{x}$ are $1 \times 2 \times 2$ tensors such that

$$
\begin{aligned}
& \nabla_{x}^{y}=\left\{\nabla_{x}^{y^{(111)}}=+1, \nabla_{x}^{y^{(121)}}=-1,0 \text { elsewhere }\right\}, \\
& \nabla_{z}^{y}=\left\{\nabla_{z}^{y^{(111)}}=-1, \nabla_{z}^{y^{(112)}}=+1,0 \text { elsewhere }\right\},
\end{aligned}
$$

which does the same within the $y$ plane. Similarly, the convolution is a 3-dimensional operation.

While $n_{t}$ is defined as an integer times $2 \pi$, which gives a degree of robustness, machine precision may preclude computation of an exact integer multiple. However, we may correctly divide the result of (5.9) by $2 \pi$ and subsequently round off to the nearest integer. The filaments are delineated as the points $\vec{x}$ for which any of the vector components in Eq. (5.9) is non-zero. Rather than "connecting the dots" to create a 3-D curve for each filament, we have chosen, for ease of visualization, to isosurface (the 3-D analogue of the 2-D contour operation) the grid using a threshold of zero; hence, the filaments in the following figures appear as tubes of constant radius rather than lines. 


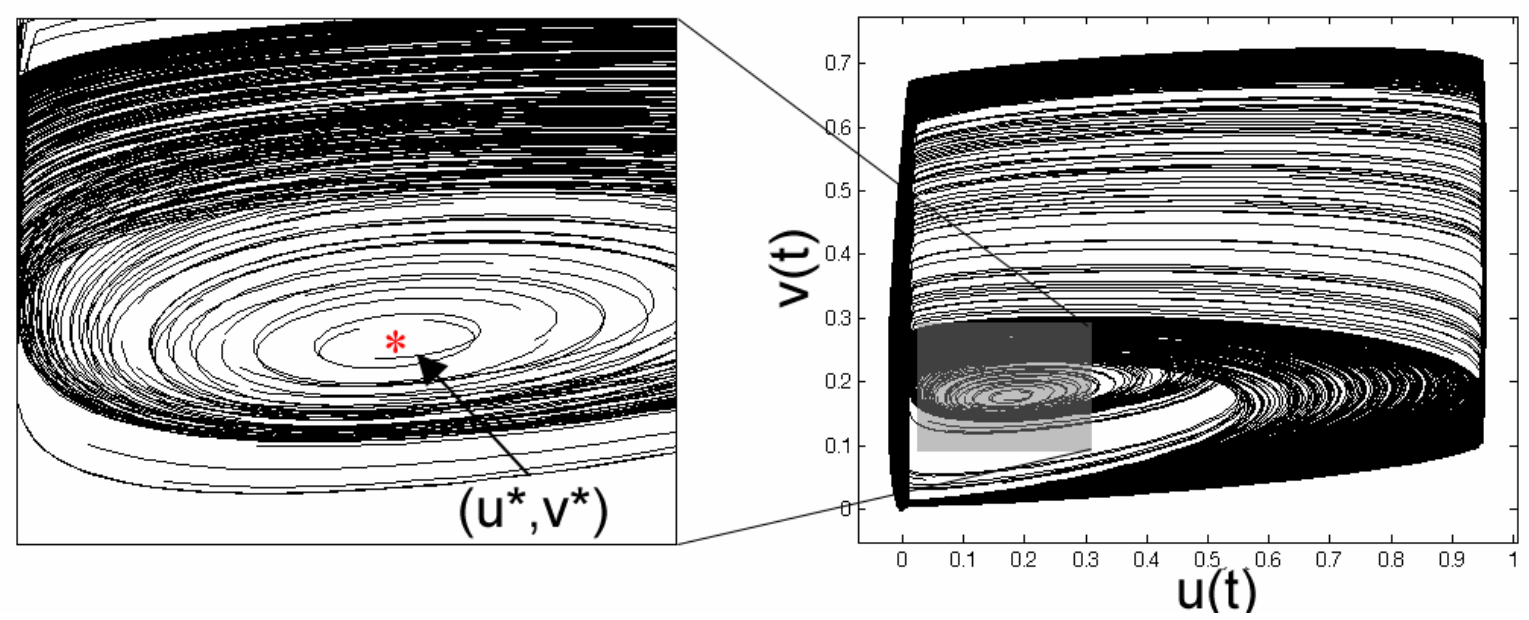

Figure 5.2: Trajectories in state space from all elements of a 2-D grid containing a spiral wave generated using the system described by Eq. (5.10) and (5.11). Inset shows the location of $\left(\mathrm{u}^{*}, \mathrm{v}^{*}\right)$.

\subsection{Results}

\subsubsection{Scroll ring behavior}

One of the more familiar examples of scroll wave reentry is the scroll ring, a rotationally symmetric loop which has been found in chemically excitable media [7] and is believed to be capable of occurring in cardiac tissue [28]. We chose this phenomenon to test our algorithm using a modified FitzHugh-Nagumo model [29,30],

$$
\begin{aligned}
& f(u, v)=f(u)-v, \\
& g(u, v)=(u-v) / \tau(u),
\end{aligned}
$$

where $f(u)$ and $\tau(u)$ are piece-wise linear functions

$$
f(u)=\left\{\begin{array}{cc}
-c_{1} u, & u<E_{1} \\
c_{2}(u-a), & E_{1} \leq u \leq E_{2}, \\
-c_{3}(u-1), & u>E_{2}
\end{array} \quad \tau(u)=\left\{\begin{array}{cc}
\tau_{1}, & u<B_{1} \\
\tau_{2}, & B_{1} \leq u \leq B_{2}, \\
\tau_{3}, & u>B_{2}
\end{array}\right.\right.
$$

where $c_{1}=4.0, c_{3}=15.0, E_{1}=0.018, \tau_{1}=\tau_{3}=0.5, \tau_{2}=16.66, B_{1}=0.01, B_{2}=0.95$, and $E_{2}=$ $\left[\left(c_{1}+c_{2}\right) E_{1}+c_{3}\right] /\left(c_{3}+c_{2}\right), a=E_{l}\left(c_{1}+c_{2}\right) / c_{2}$ to insure continuity of $f(u)$. For this instance, we set $c_{2}=0.88$ and used a $120 \times 120 \times 120$ monodomain spatial grid with a spatial discretization of 0.5 spatial units (s.u.). The simulations used a simple forward Euler method for time integration with a temporal discretization of 0.01 time units $(t . u$.), with no-flux boundary conditions on the spatial grid. For this model, $\left(u^{*}, v^{*}\right)$ was chosen as $(0.175,0.177)$, as shown in Figure 5.2. 
Figure 5.3A shows a cross-section through a vortex scroll wave, in gold, with the calculated filament ring superimposed in green. We can clearly see how the wave rotates around and through the filament half-loop to start the next reentrant loop. The scroll ring filament shrinks with time while drifting along its symmetry axis as predicted theoretically [31]; in Fig. 5.3B, we also observe the final closure of the filament ring when reentry terminates.

\subsubsection{Complex fibrillatory behavior}

It is believed that the emergence and subsequent proliferation of scroll waves underlie arrhythmias such as VF [18,32-35]. Such behavior is characterized by extreme spatiotemporal turbulence. As a demonstration of the ability of the algorithm to represent such complex behavior, we used a model developed by Aliev and Panfilov [36]

$$
\begin{aligned}
& f(u, v)=-\alpha u(u-\beta)(u-1)-u v, \\
& g(u, v)=\left(\sigma+\frac{\mu_{1} v}{u+\mu_{2}}\right)(-v-\alpha u(u-\beta-1)),
\end{aligned}
$$

where $\beta=0.15, \sigma=0.002, \mu_{1}=0.2, \mu_{2}=0.3$, and $\alpha=40$. The spatial grid is a $120 \times 120 \times 16$ mesh with a 1.0 s.u. discretization; the simulation parameters and boundary conditions remained the same as those used for $(5.11)$. The origin $\left(u^{*}, v^{*}\right)$ was chosen as $(0.0235,1.06)$ in this model, as illustrated in Figure 5.4.

In this model, the filament dynamics become unstable with time due to negative filament tension [14], causing a single scroll wave to break up into multiple wavelets for a given value of $\alpha$, provided the excitable medium is sufficiently thick [37]. Figure 5.5 shows an example of unstable scroll wave reentry. The reentry was initiated as a wavebreak with a slight slant in the $\mathrm{z}$ direction $(t=0 t . u$. $)$. This perturbation combined with negative filament tension causes the filament to curve and break into three pieces on the lower surface $(t=174)$. Each of the new filaments further spawns new filament breaks until full fibrillatory activity is present $(t=800)$.

\subsubsection{Comparison between two-variable and time-series state space}

We used the model described in Eq. (5.12) to compare the filament motion generated by the two-variable state space $(u(t), v(t))$ and the time-series state space $(u(t), u(t+\tau))$; an example of the trajectories from the two state spaces is shown in Figure 5.6. The spatial grid was 

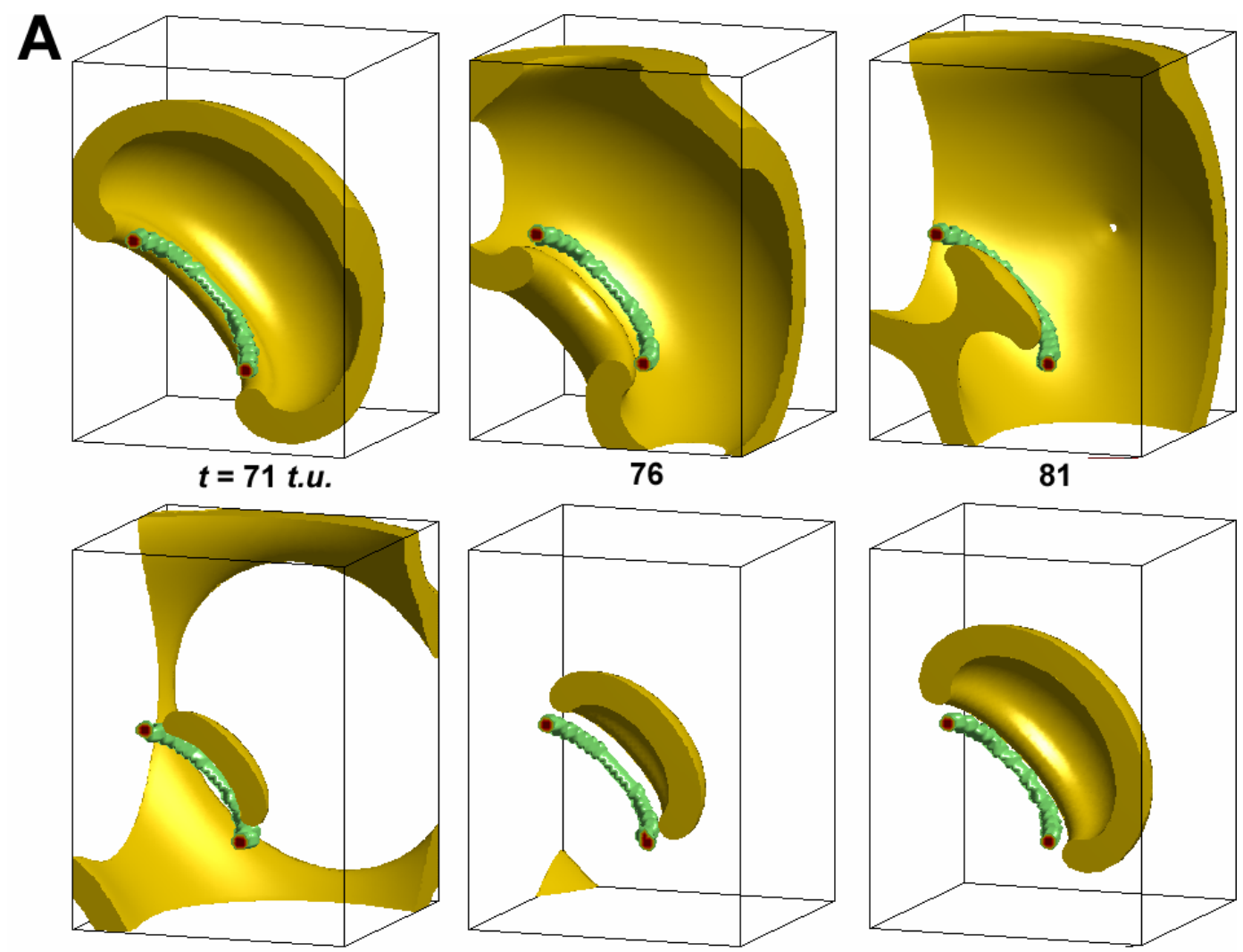

85
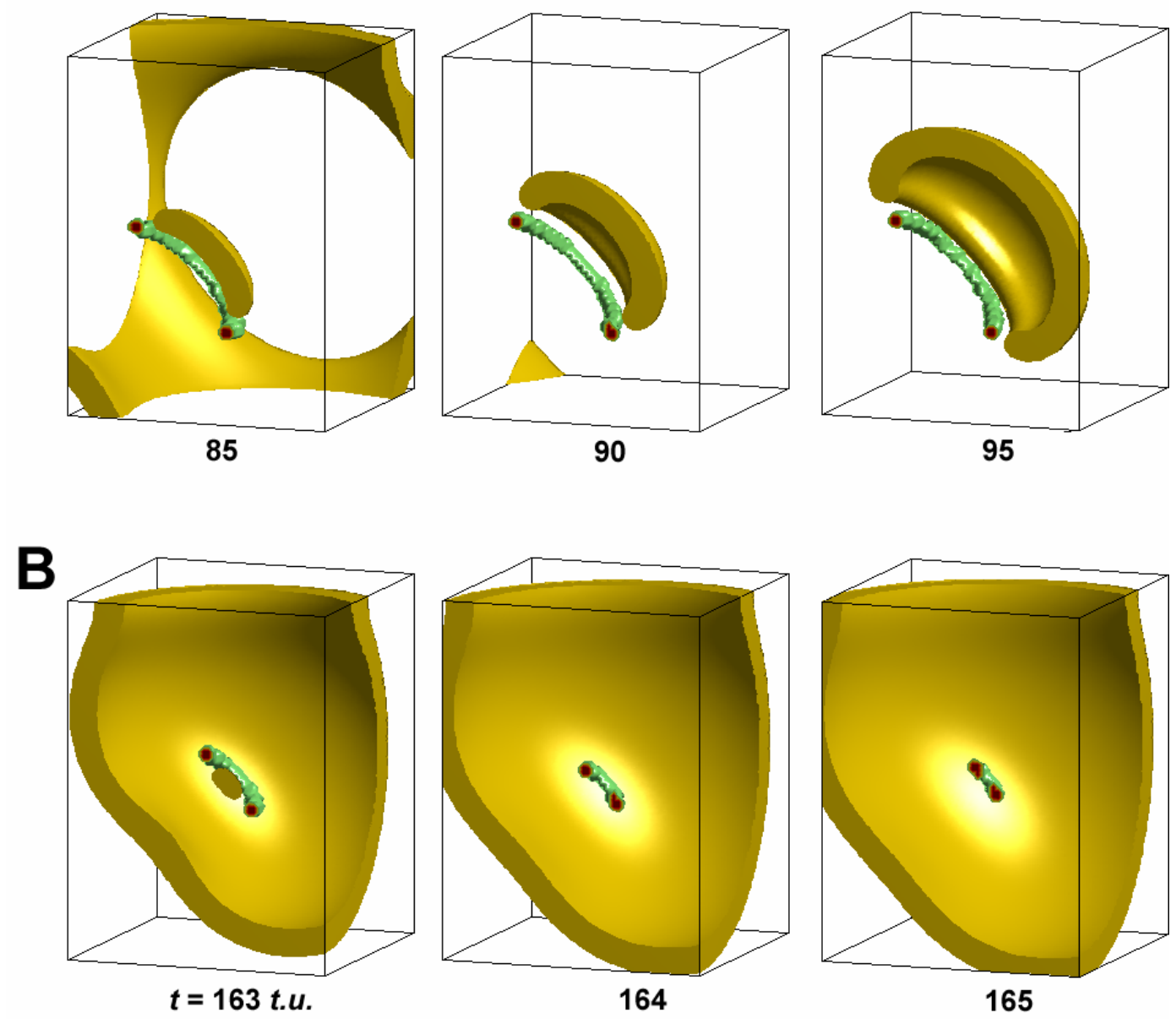

Figure 5.3: (A) Snapshots of a cross-section through a simulated vortex ring generated by the system described by Eq. (5.10) and (5.11) with the wave (gold) isosurfaced at $75 \%$ of maximal activation amplitude. The filament as calculated by the curl algorithm is shown in green. The number underneath each frame is the time of the snapshot in time units. (B) Snapshots during the spontaneous annihilation of the vortex ring. Time is in time units (t.u.). 


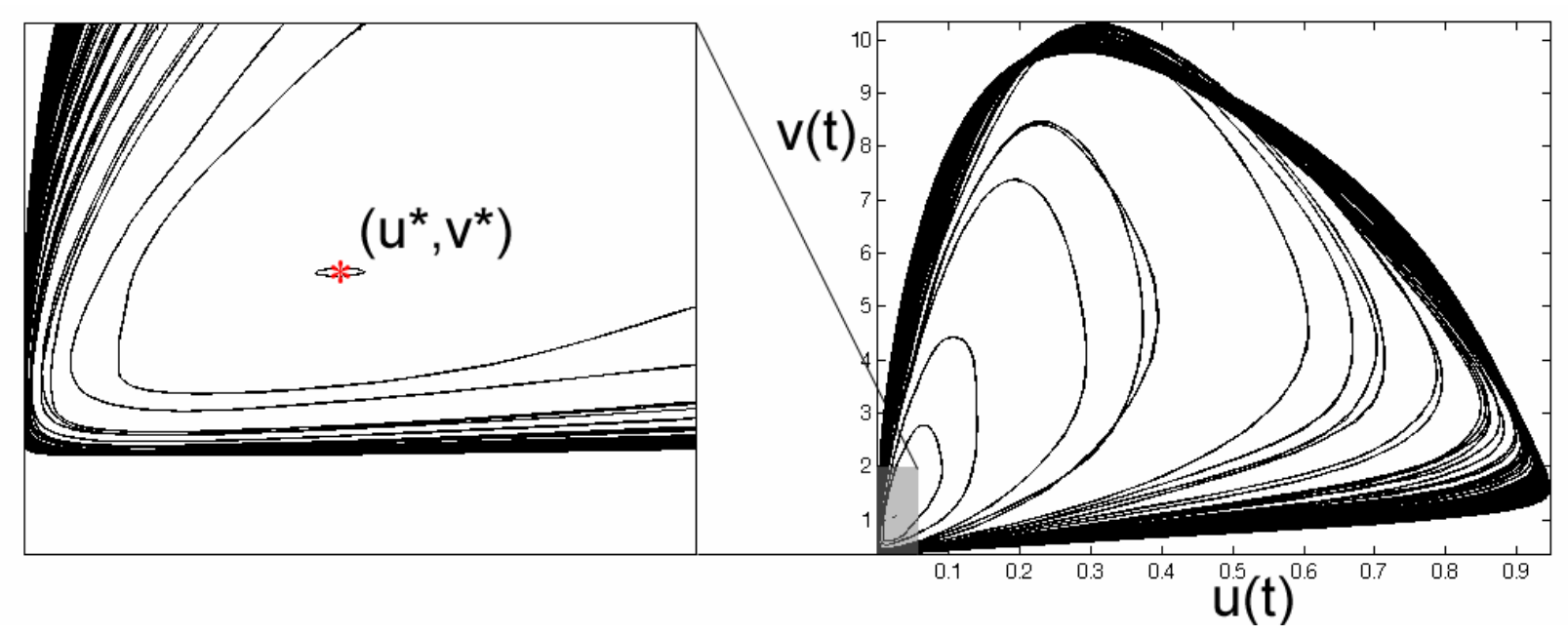

Figure 5.4: Trajectories in state space from all elements of a 2-D grid containing a spiral wave generated using the system described by Eq. (5.12). Inset shows the location of $\left(\mathrm{u}^{*}, \mathrm{v}^{*}\right)$.
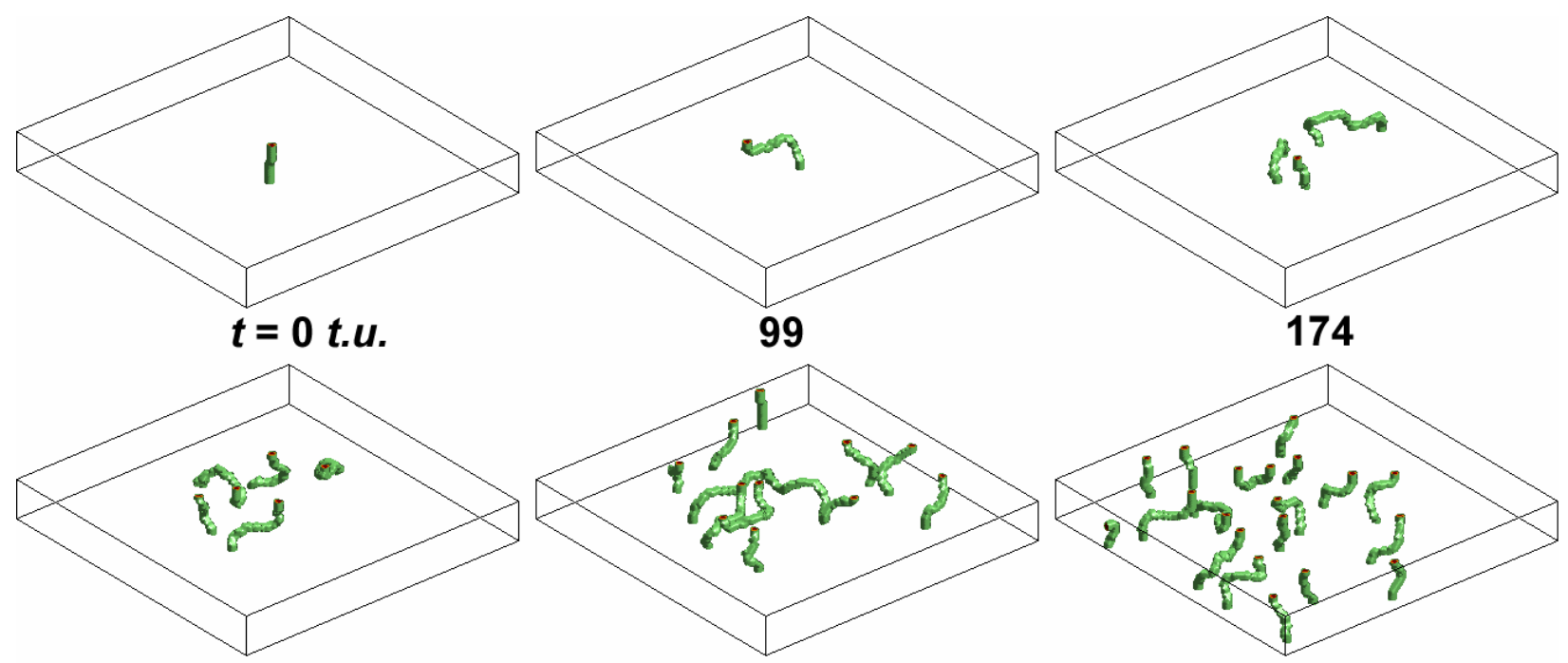

220

308

800

Figure 5.5: Three-dimensional snapshots of vortex filaments generated by the system described by Eq. (5.12) during unstable scroll wave reentry. The number underneath is the time of the snapshot in time units. Time is in time units (t.u.). 

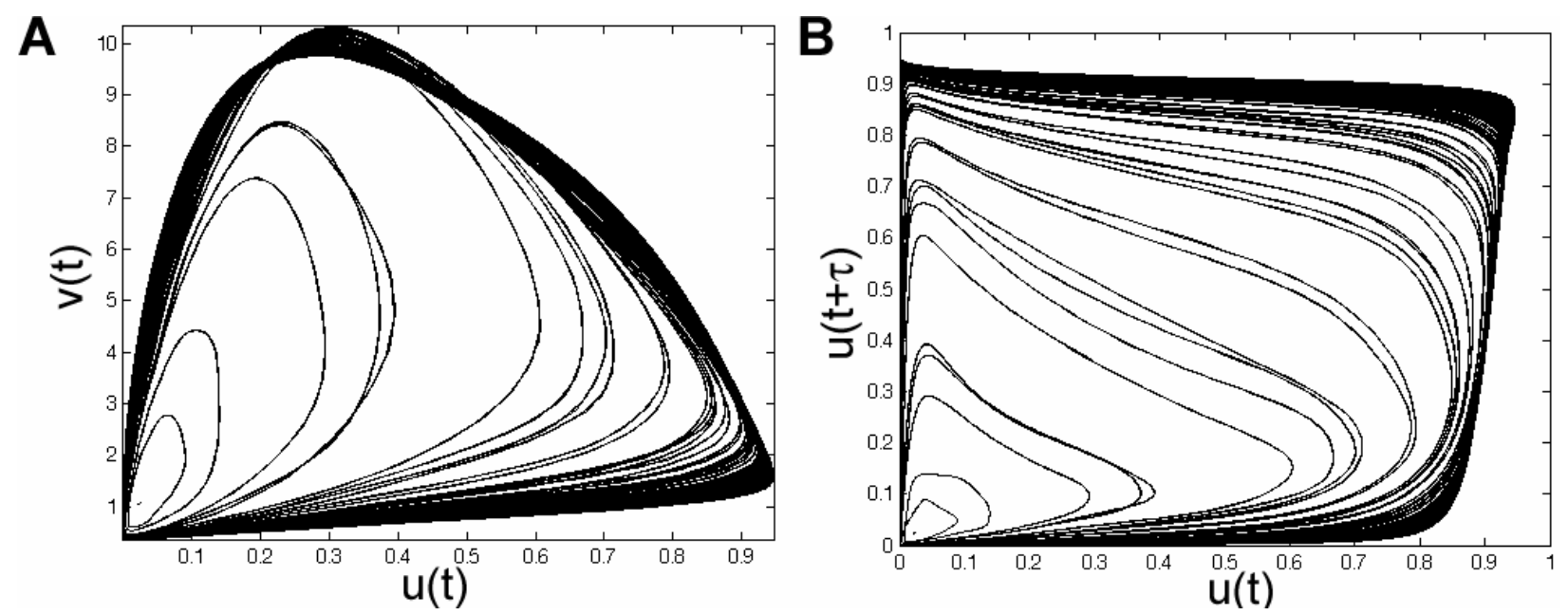

Figure 5.6: Phase portraits generated by the system described by Eq. (5.12) for (A) two variable state space and (B) time-series state space. $\tau$ is set at 1.26 t.u in (B).

a $120 \times 120 \times 32$ mesh with spatial and temporal discretizations of 0.5 s.u. and 0.01 t.u., respectively, and $\tau$ was set at 1.26 t.u., approximately the duration of the action potential upstroke. A scroll wave was initiated by the same process mentioned above, and the simulation was run until shortly before the original filament collided with the bottom surface, breaking it into two new filaments. Subsequent surface-filament collisions produced new singularity pairs upon the surface, where each singularity is the terminating end of a filament. Each of these singularities was tracked using the topological charge algorithm applied to the phase field derived from either the two-variable state space or the time-series state space. We measured the time-of-life of each of the observed singularities over a 432 t.u. time span, sampling the data every 1.08 t.u. .

Table 5.1 displays the results. Eleven singularities were observed on the surface during the course of the simulation. The time-of-life of the singularities ranged from 9.72 to 224.64 t.u., dependent upon the underlying filament behavior; a singularity would vanish when the filament moved from the top surface to one of the other bounding surfaces. Six of the eleven singularities were observed over the same time interval for the two phase methodologies; for the remaining five cases, the discrepancy was one sampling interval. The RMS error between the two methods was calculated over that that period of time during which the singularities appeared in both methodologies, and averaged 0.23 s.u., ranging from 0.16 to 0.31 s.u.. 
Table 5.1: Measured time-of-life and error between wavetip trajectories on the top surface produced by the two-variable state space $(2 \mathrm{~V})$ and time-series state space reconstruction (TS). Time-of-life measured in time units (t.u.), error measured in space units (s.u.).

\begin{tabular}{c|c|c}
\hline $\begin{array}{c}\text { Singularity } \\
\text { number }\end{array}$ & $\begin{array}{c}\text { Time-of-life }(t . u .) \\
2 \mathrm{~V} / \mathrm{TS}\end{array}$ & RMS Error (s.u.) \\
\hline 1 & $117.72 / 117.72$ & $0.21 \pm 0.26$ \\
2 & $224.64 / 224.64$ & $0.16 \pm 0.24$ \\
3 & $10.8 / 9.72$ & $0.22 \pm 0.36$ \\
4 & $72.36 / 72.36$ & $0.22 \pm 0.26$ \\
5 & $18.36 / 19.44$ & $0.25 \pm 0.32$ \\
6 & $15.12 / 14.04$ & $0.31 \pm 0.31$ \\
7 & $115.56 / 114.48$ & $0.27 \pm 0.31$ \\
8 & $144.72 / 144.72$ & $0.17 \pm 0.25$ \\
9 & $56.16 / 56.16$ & $0.29 \pm 0.27$ \\
10 & $77.76 / 77.76$ & $0.25 \pm 0.28$ \\
11 & $29.16 / 29.16$ & $0.17 \pm 0.25$
\end{tabular}



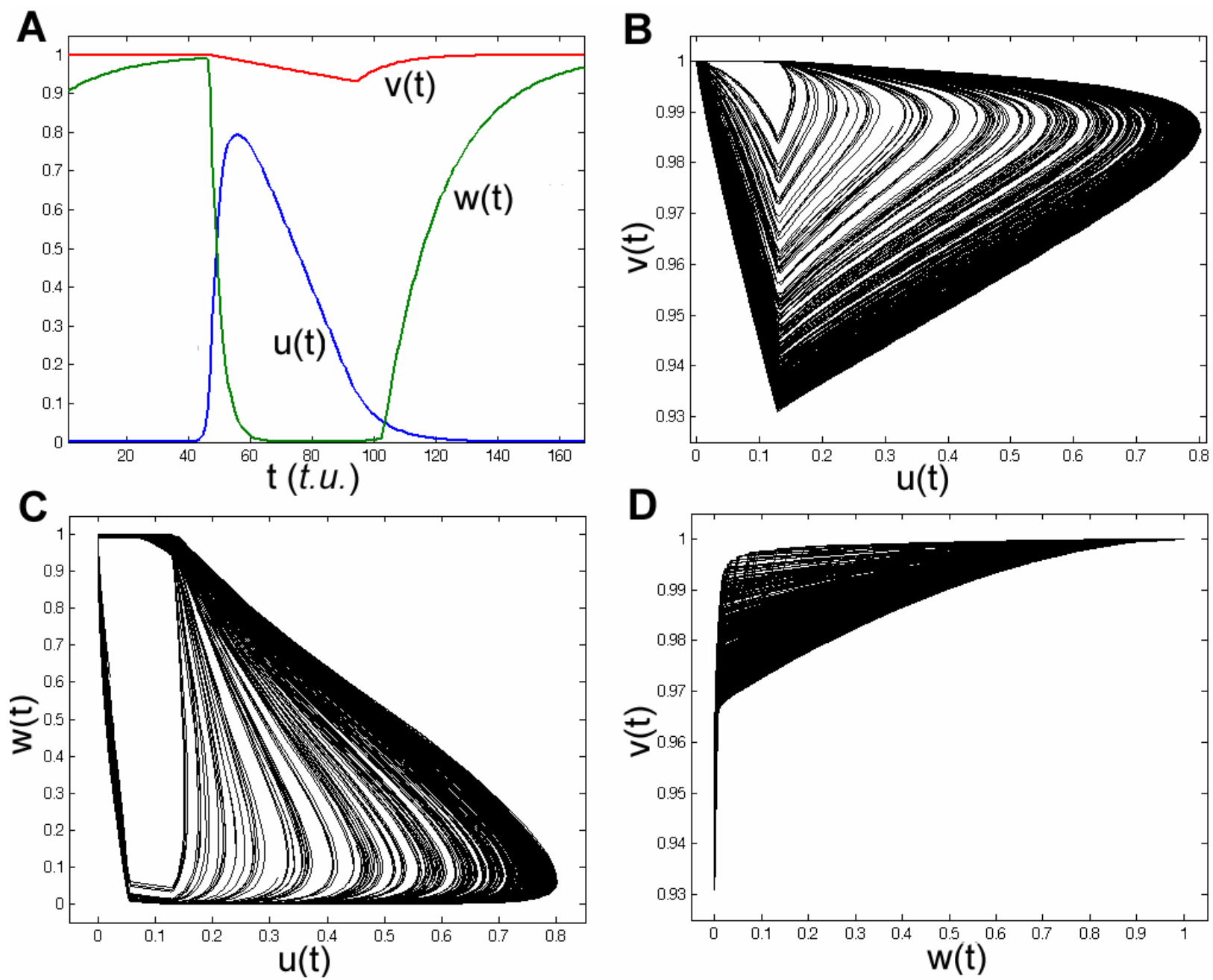

Figure 5.7: (A) An example of the waveforms $v(t), u(t)$, and $w(t)$ produced by the system described by Eq (5.13). (B) $(u(t), v(t))$ state space. (C) $(u(t), w(t))$ state space. (D) $(w(t), v(t))$ state space.

\subsubsection{Three-variable reaction-diffusion model}

Up until this point, we have used a two-variable model of cardiac activity, which serves as a computationally inexpensive caricature of action potential morphology. However, more complex models exist which attempt to realistically duplicate the ionic currents which are responsible for the dynamic properties of the cardiac action potential; each current is represented by a differential equation governing the change in ion concentration (see [18,38,39] for examples). While it is trivial to construct a two-dimensional state space when only two parameters are available, we now turn our attention to the issue of uniquely determining phase when a multi-variable model is used. 
We have chosen to use a three-variable model developed by Fenton and Karma designed to reproduce the spiral wave behavior of more complex ionic models while remaining numerically simple for 3-D applications [40]. The equations are

$$
\begin{aligned}
& \partial u / d t=D \nabla^{2} u-J_{f i}(u, v)-J_{s o}(u)-J_{s i}(u, w), \\
& \partial v / d t=H\left(u_{c}-u\right)(1-v) / \tau_{v}^{-}(u)-H\left(u-u_{c}\right) v / \tau_{v}^{+}, \\
& \partial w / d t=H\left(u_{c}-u\right)(1-w) / \tau_{w}^{-}-H\left(u-u_{c}\right) w / \tau_{w}^{+},
\end{aligned}
$$

where $u$ is the dimensionless transmembrane potential, $D=0.001, v$ and $w$ are gating variables responsible for inactivation and reactivation of the currents $J_{f i}, J_{s o}$, and $J_{s i}$, and

$$
\begin{aligned}
& J_{f i}(u, v)=-\frac{v}{\tau_{d}} H\left(u-u_{c}\right)(1-u)\left(u-u_{c}\right), \\
& J_{s o}(u)=-\frac{u}{\tau_{o}} H\left(u_{c}-u\right)+\frac{1}{\tau_{r}} H\left(u-u_{c}\right), \\
& J_{s i}(u, w)=-\frac{w}{2 \tau_{s i}}\left(1+\tanh \left[k\left(u-u_{c}^{s i}\right)\right]\right), \\
& \tau_{v}^{-}(u)=H\left(u-u_{v}\right) \tau_{v 1}^{-}+H\left(u_{v}-u\right) \tau_{v 2}^{-},
\end{aligned}
$$

and $H(x)$ is the standard Heavside step function. We have chosen to use the parameter values which were used to fit the model to the modified Beeler-Reuter (MBR) restitution curve. Because we wished to examine a near-stationary case for the sake of simplicity, we set the conductance parameter to 2.4 , for which the 2-D isotropic wavetip motion is circular. The other values of the parameters for this configuration are provided in [40], so we omit them here. An example of the waveforms produced by the three variables is shown in Figure 5.7A.

We performed simulations on a $200 \times 200$ grid with spatial and temporal discretizations of 0.0015 s.u. and 0.025 t.u., respectively. State space trajectories are shown for $u$ versus $v$ (Fig. 5.7B), $u$ versus $w$ (Fig. 5.7C), and $w$ versus $v$ (Fig. 5.7D). We observed that for $(u, v)$ and $(u, w)$ state space, there exists a clear center of rotation, but for $(w, v)$ state space, no such center is present. By choosing $\left(u^{*}, v^{*}\right)$ to be $(0.127,0.990)$ and $\left(u^{*}, w^{*}\right)$ to be $(0.08,0.85)$, we found that the spiral wave traced out a circular pattern on the grid surface for both state spaces, as shown in Fig. 5.8 . 


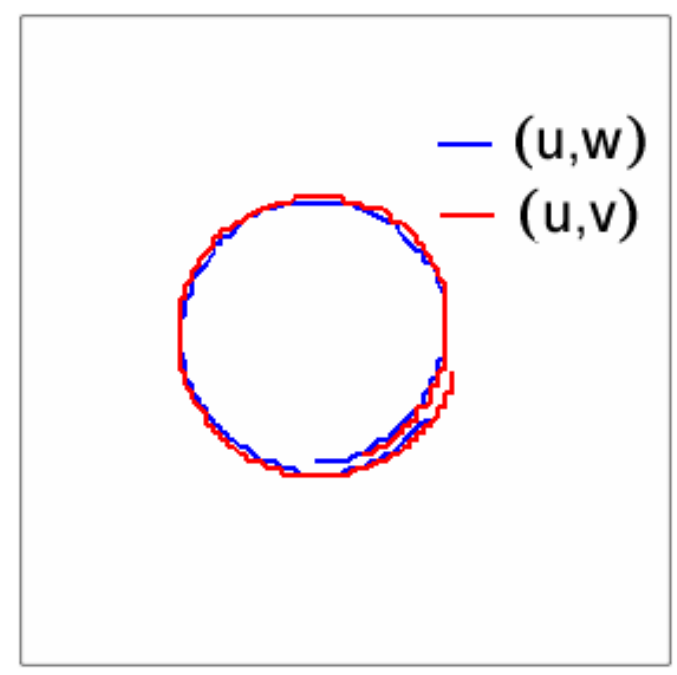

Figure 5.8: Wavetip path traced out by the system described by Eq. (5.14) for $(u(t), v(t))$ and ( $u(t), w(t))$ state spaces.

\subsection{Discussion}

The use of phase to localize phase singularities in both numerically simulated studies and experimental preparations has been shown previously $[3,21,24,41]$. The use of topological charge lends itself as a robust methodology to detect these singular points [21]. In this paper, we have shown that the same principle may be easily extended to detect filaments in three dimensions in models of cardiac reentry.

\subsubsection{Properties of the Nabla kernels}

It is worth mentioning that the Nabla kernels perform two functions. First, the kernels are derivative operators similar to the Prewitt operators used in image processing [42], which are appropriate for evaluating the differential form of the curl operation, as in Eq. (5.5). Second, the kernels function as a compact summation operation over a discretized path. This feature is needed at the singularity itself, where differentiability fails, and evaluation of the path integral in Eq. (5.4) is necessary to obtain $n_{t}$ rather than an indeterminate value. For the Nabla kernels shown in (5.8), the path length is 8 units (over a 3 x 3 square), which will produce a singularity at one pixel. While small lengths are desirable for optimal localization, paths of various lengths may be constructed simply by altering the kernel size and elements; for example, a path of length 12 (a 4 x 4 square) is defined by 


$$
\nabla_{x}^{z}=\left[\begin{array}{ccc}
+1 & 0 & -1 \\
+1 & 0 & -1 \\
0 & 0 & 0
\end{array}\right], \text { and } \nabla_{y}^{z}=\left[\begin{array}{ccc}
-1 & -1 & 0 \\
0 & 0 & 0 \\
+1 & +1 & 0
\end{array}\right]
$$

\subsubsection{The issue of state space selection}

For a stationary scroll wave modeled with a two-variable system, the origin may be selected by examination of the $(u, v)$ state space portrait, since the trajectories all encircle a single point (Figs. 5.2 and 5.4). This is the case even for systems with low excitability; in the model described by Eqs. (5.10), for $c_{2}=0.75$, the origin was found to be $\left(2.25 \times 10^{-3}, 2.25 \times 10^{-3}\right)$ and resulted in a filament at the center of a nearly quiescent core with the scroll wave rotating at some distance around it.

Using our two-variable models, we have examined the effect of using two methods to construct the state space in order to define phase: using the two model parameters as independent variables (Eq.(5.2)) or using time-delay embedding to create a time-shifted variable from one of the parameters (Eq. (5.3)). In the majority of cases (6 out of 11), the observed surface singularities appeared and terminated during the same interval. In the other cases, the variation was within one sampling interval (1.08 t.u.). The spatial difference in localization was also fairly small: on a spatial grid with a 0.5 s.u. discretization, the average error was smaller than the discretization. The similarity of the results suggests that one variable is indeed sufficient to obtain the phase field data, a conclusion also reached by Iyer and Gray, who examined 2-D spiral wave simulations [41]. However, in their simulations, they performed their comparison on a stationary BAR model, whereas we utilized a non-stationary system in which we would expect the average error to be somewhat greater.

Even though such simple models remain useful, more physiologically realistic models have come to the forefront for the investigation of cardiac dynamics. These models entail the addition of parameters to represent the individual ionic currents present in the cell, with varying degrees of complexity. The question now becomes, which of these parameters do we use to construct the state-space? The answers lies in recognizing the desired characteristics of the trajectories in state space, i.e., phase is uniquely defined if the trajectories revolve in a consistent direction in state space around a given origin during the course of a single wave rotation. In nonlinear dynamics literature, the analytic signal concept (based on the Hilbert transform) may 
be used to define a unique phase of a signal by creating a signal with a $\pi / 2$ phase lag [43]. It turns out that one property of the original signal and the Hilbert transformed signal is that they are orthogonal over the infinite interval. Hence, in order to uniquely determine phase, the model parameters should be chosen such that they are as close to orthogonal to one another as possible over the duration of the signal [44]. With this in mind, we can now see why the $(v, w)$ state space in Fig. 5.7D was an inappropriate choice for singular point detection: looking at Fig. 5.7A, we observe that $v(t)$ and $w(t)$ are strongly correlated as compared to $u(t)$ with respect to both $v(t)$ and $w(t)$. A calculation of the cross-correlation indicates that $u(t)$ and $w(t)$ would be the best choice for this model in terms of orthogonality (that is, minimal cross-correlation).

Preliminary experimental results indicate that a useful state space may be created using the optically recorded $\mathrm{Ca}^{2+}$ and transmembrane potential signals [45]. However, even with explicit knowledge of all the parameters, an investigator can still opt to use a single observable variable (such as the transmembrane potential) to reconstruct the state space by taking advantage of Taken's embedding theorem.

\subsubsection{Future directions}

Finally, it is worth noting that despite the variety of methods available to the researcher for singularity and filament localization (as described in the Introduction), a universally accepted "gold standard" methodology does not exist. Iyer and Gray [41] have performed a partial analysis, comparing the phase analysis method for 2-D simulations against isochrone and isopotential map methods, and found that the phase analytical method was consistent with these

other methods. Therefore, it stands to reason that the approach described here for localization of 3-D filaments should also be consistent with other methods described above, at least on a qualitative level. While a comprehensive quantitative comparison of the accuracy of topological approach relative to the other methodologies is beyond the scope of the present work, such a study would be extremely useful to the cardiac community.

\subsubsection{Limitations}

The topological charge segment of the algorithm, Eq. (5.9), will localize the singular values located in any given phase field. However, we must first insure that the phase field is in 
fact accurate. As mentioned before, the choice of the origin $\left(\mathrm{u}^{*}, \mathrm{v}^{*}\right)$ in state space is critical for the proper calculation of phase in Eq. (5.2), and must be determined beforehand.

Recall that the only requirement for the detection of a singular point is a net $2 \pi$ phase distribution of the surrounding spatial points about $\left(\mathrm{u}^{*}, \mathrm{v}^{*}\right)$ when mapped into state space. The choice of origin may differ from model to model, as we have seen above, but this requirement does not, which actually provides the criterion for whether the choice of origin is an appropriate one. While this is straightforward for a stationary or near-stationary wave, for rapidly moving scroll waves, we may need to compromise by finding the origin which is encircled by most, rather than all, of the trajectories. Iyer and Gray [41] addressed this issue in part by localizing the phase singularity of a non-stationary 2-D spiral wave. They found that the singularity could be localized to within the core region even under the presence of $10 \%$ added noise, although the error was greater than that for a stationary spiral wave. Clearly, the formulation of an algorithm for selecting the optimal origin for a non-stationary wave deserves more thorough study.

Another issue is that of localization precision. As it stands, the topological charge algorithm is constrained to the discretization of the local grid when computing the convolution operation. Hence, the localization of the singular points is as precise as the grid spacing, which may be problematic when dealing with numerically sensitive filament properties, such as velocity and twist. This may dealt with by using a finer grid discretization, but this solution may be computationally expensive. Another solution is to find the intersection of two different isophase contours in the phase field. While this solution does not use Eq. (5.4), the fact that the phase contours intersect at the filament stems from topological considerations.

\subsection{Acknowledgments}

We would like to thank Rubin Aliev, Rick Gray, Michael Miga, and the reviewers for their helpful comments during the preparation of this manuscript. This work was supported in part by NIH grant R01HL58241 and a graduate research fellowship from the UNCF-Merck Science Initiative. 


\subsection{References}

[1] Winfree, A. T., When Time Breaks Down: The Three-Dimensional Dynamics Of Electrochemical Waves And Cardiac Arrhythmias. Princeton: Princeton University Press, 1987.

[2] Gray, R. A. and Jalife, J., "Spiral waves and the heart." Int J Bifurc Chaos, vol. 6, no. 3, pp. 415-435, 1996.

[3] Gray, R. A., Pertsov, A. M., and Jalife, J., "Spatial and temporal organization during cardiac fibrillation." Nature, vol. 392, no. 6671, pp. 75-78, 1998.

[4] Lin, S.-F., Roth, B. J., and Wikswo, J. P., Jr., "Quatrefoil reentry in myocardium: An optical imaging study of the induction mechanism." J Cardiovasc Electrophysiol, vol. 10, no. 4, pp. 574-586, 1999.

[5] Banville, I., Gray, R. A., and Ideker, R. E., "Shock-induced figure-eight reentry in the whole heart: An optical mapping study." Circ Res, vol. 85, no. 8, pp. 742-752, 1999.

[6] Pertsov, A., Vinson, M., and Müller, S. C., "Three-dimensional reconstruction of organizing centers in excitable chemical media." Physica D, vol. 63, no. 1-2, pp. 233-240, 1993.

[7] Winfree, A. T., Caudle, S., Chen, G., McGuire, P., and Szilagyi, Z., "Quantitative optical tomography of chemical waves and their organizing centers." Chaos, vol. 6, no. 4, pp. 617-626, 1996.

[8] Gray, R. A., Jalife, J., Panfilov, A., Baxter, W. T., Cabo, C., Davidenko, J. M., and Pertsov, A. M., "Nonstationary vortexlike reentrant activity as a mechanism of polymorphic ventricular tachycardia in the isolated rabbit heart." Circulation, vol. 91, no. 9, pp. 2454-2469, 1995.

[9] Efimov, I. R., Sidorov, V., Cheng, Y., and Wollenzier, B., "Evidence of threedimensional scroll waves with ribbon-shaped filament as a mechanism of ventricular tachycardia in the isolated rabbit heart." J Cardiovasc Electrophysiol, vol. 10, no. 11, pp. 1452-1462, 1999.

[10] Biktashev, V. N., Holden, A. V., Mironov, S. F., Pertsov, A. M., and Zaitsev, A. V., "Three-dimensional aspects of re-entry in experimental and numerical models of ventricular fibrillation." Int J Bifurc Chaos, vol. 9, no. 4, pp. 694-704, 1999.

[11] Zaitsev, A. V., Berenfeld, O., Mironov, S. F., Jalife, J., and Pertsov, A. M., "Distribution of excitation frequencies on the epicardial and endocardial surfaces of fibrillating ventricular wall of the sheep heart." Circ Res, vol. 86, no. 4, pp. 408-417, 2000.

[12] Chen, P.-S., Wolf, P. D., Dixon, E. G., Danieley, N. D., Frazier, D. W., Smith, W. M., and Ideker, R. E., "Mechanism of ventricular vulnerability to single premature stimuli in open-chest dogs." Circ Res, vol. 62, no. 6, pp. 1191-1209, 2000. 
[13] Baxter, W. T., Mironov, S. F., Zaitsev, A. V., Jalife, J., and Pertsov, A. M., "Visualizing excitation waves inside cardiac muscle using transillumination." Biophys J, vol. 80, no. 1, pp. 516-530, 2001.

[14] Biktashev, V. N., Holden, A. V., and Zhang, H., "Tension of organizing filaments of scroll waves." Phil Trans R Soc Lond A, vol. 347, no. 1685, pp. 611-630, 1994.

[15] Vinson, M., "Interactions of spiral waves in inhomogeneous excitable media." Physica D, vol. 116, no. 3, pp. 313-324, 1998.

[16] Berenfeld, O. and Pertsov, A. M., "Dynamics of intramural scroll waves in threedimensional continuous myocardium with rotational anisotropy." J Theor Biol, vol. 199 pp. 383-394, 1999.

[17] Pertsov, A. M., Wellner, M., Vinson, M., and Jalife, J., "Topological constraint on scroll wave pinning." Phys Rev Lett, vol. 84, no. 12, pp. 2738-2741, 2000.

[18] Fenton, F. and Karma, A., "Vortex dynamics in three-dimensional continuous myocardium with fiber rotation: Filament instability and fibrillation." Chaos, vol. 8, no. 1, pp. 20-47, 1998.

[19] Qu, Z., Kil, J., Xie, F., Garfinkel, A., and Weiss, J. N., "Scroll wave dynamics in a threedimensional cardiac tissue model: roles of restitution, thickness, and fiber rotation." Biophys J, vol. 78, no. 6, pp. 2761-2775, 2000.

[20] Beaumont, J., Davidenko, N., Davidenko, J. M., and Jalife, J., "Spiral waves in twodimensional models of ventricular muscle: Formation of a stationary core." Biophys J, vol. 75 , no. 1, pp. 1-14, 1998.

[21] Bray, M.-A., Lin, S.-F., Aliev, R. R., Roth, B. J., and Wikswo, J. P., Jr., "Experimental and theoretical analysis of phase singularity dynamics in cardiac tissue." J Cardiovasc Electrophysiol, vol. 12, no. 6, pp. 716-722, 2001.

[22] Takens, F., "Detecting strange attractors in turbulence.," in Rand, D. A. and Young, L.-S. (eds.) Dynamical Systems and Turbulence Berlin: Springer-Verlag, 1981, pp. 366-381.

[23] Fraser, A. M. and Swinney, H. L., "Independent coordinates for strange attractors from mutual information." Physical Review A, vol. 33, no. 2, pp. 1134-1140, 1986.

[24] Gray, R. A. and Jalife, J., "Video imaging of atrial defibrillation.," in Zipes, D. P. and Jalife, J. (eds.) Cardiac Electrophysiology: From Cell to Bedside 3rd ed. Philadelphia: W.B. Saunders, 1999, pp. 432-439.

[25] Goryachev, A. and Kapral, R., "Spiral waves in chaotic systems." Phys Rev Lett, vol. 76, no. 10, pp. 1619-1622, 1996.

[26] Mermin, N. D., "The topological theory of defects in ordered media." Rev Mod Phys, vol. 51, no. 3, pp. 591-648, 1979. 
[27] Hyman, J. M. and Shashkov, M., "Natural discretizations for the divergence, gradient, and curl on logically rectangular grids." Computers Math Applic, vol. 33, no. 4, pp. 81104, 1997.

[28] Aliev, R. R. and Panfilov, A. V., "Modeling of heart excitation patterns caused by a local inhomogeneity." J Theor Biol, vol. 181, no. 1, pp. 33-40, 1996.

[29] FitzHugh, R., "Impulses and physiological states in theoretical models of nerve membrane." Biophys J, vol. 1, no. 6, pp. 445-466, 1961.

[30] Vinson, M. and Pertsov, A., "Dynamics of scroll rings in a parameter gradient." Phys Rev E, vol. 59, no. 3, pp. 2764-2771, 1999.

[31] Panfilov, A. V. and Pertsov, A. M., "Vortex ring in a three-dimensional medium described by reaction-diffusion equations." Dokl Biophys, vol. 274, no. 6, pp. 58-1, 1984.

[32] Frazier, D. W., Wolf, P. D., Wharton, J. M., Tang, A. S., Smith, W. M., and Ideker, R. E., "Stimulus-induced critical point. Mechanism for electrical initiation of reentry in normal canine myocardium." J Clin Invest, vol. 83, no. 3, pp. 1039-1052, 1989.

[33] Winfree, A. T., "Electrical turbulence in three-dimensional heart muscle." Science, vol. 266, no. 5187, pp. 1003-1006, 1994.

[34] Pogwizd, S. M. and Corr, P. B., "Mechanisms underlying the development of ventricular fibrillation during early myocardial ischemia." Circ Res, vol. 66, no. 3, pp. 672-695, 1990.

[35] Panfilov, A. V., "Spiral breakup as a model of ventricular fibrillation." Chaos, vol. 8, no. 1, pp. 57-64, 1998.

[36] Aliev, R. R. and Panfilov, A. V., "A simple two-variable model of cardiac excitation." Chaos, Solitons, \& Fractals, vol. 7, no. 3, pp. 293-301, 1996.

[37] Gray, R. A. and Jalife, J., "Ventricular fibrillation and atrial fibrillation are two different beasts." Chaos, vol. 8, no. 1, pp. 65-78, 1998.

[38] Beeler, G. W. and Reuter, H., "Reconstruction of the action potential of ventricular myocardial fibres." J Physiol (Lond), vol. 268, no. 1, pp. 177-210, 1977.

[39] Luo, C. H. and Rudy, Y., "A dynamic model of the cardiac ventricular action potential. I. Simulations of ionic currents and concentration changes." Circ Res, vol. 74, no. 6, pp. 1071-1096, 1994.

[40] Fenton, F. and Karma, A., "Fiber-rotation-induced vortex turbulence in thick myocardium." Phys Rev Lett, vol. 81, no. 2, pp. 481-484, 1998.

[41] Iyer, A. N. and Gray, R. A., "An experimentalist's approach to accurate localization of phase singularities during reentry." Ann Biomed Eng, vol. 29, no. 1, pp. 47-59, 2001. 
[42] Gonzales, R. C. and Woods, R. E., Digital Image Processing. Addison-Wesley Publishing Co, 1993.

[43] Pikovsky, A. S., Rosenblum, M. G., Osipov, G. V., and Kurths, J., "Phase synchronization of chaotic oscillators by external driving." Physica D, vol. 104, no. 3-4, pp. 219-238, 1997.

[44] Yalcinkaya, T. and Lai, Y.-C., "Phase characterization of chaos." Phys Rev Lett, vol. 79, no. 20, pp. 3885-3888, 1997.

[45] Gray, R. A., Wikswo, J. P., Jr., Lin, S.-F., and Baudenbacher, F. Phase mapping using both transmembrane potential and calcium. Pacing and Clinical Electrophysiology 23(4 Part II), 608. 2000. 


\title{
CHAPTER VI
}

Bifurcations And An Interaction Potential In Decaying Vortex FILAMENT PAIRS

\author{
Mark-Anthony Bray ${ }^{1}$ and John P. Wikswo ${ }^{2}$
}

${ }^{1}$ Department of Biomedical Engineering

${ }^{2}$ Department of Physics and Astronomy

Vanderbilt University

Nashville, TN 37235,

This manuscript has been submitted for publication in Physical Review Letters. 


\subsection{Abstract}

The dynamics of vortex filament-filament interactions remain poorly understood in hydrodynamics, superconductors, and excitable media. Cardiac tissue is of particular interest because the creation and interaction of 3-D line defects (filaments) are believed to underlie lethal arrhythmias. We replicate an experimentally observed reentrant filament configuration using a numerical model of the Belousov-Zhabotinsky (BZ) reaction to simulate a pair of adjacent circular filaments (scroll rings) with common symmetry axes and varying initial radii and separation distances. The interaction properties are quantified in terms of the scroll-ring lifetime $\left(T_{L}\right)$ and direction of initial velocity $\left(V_{0}\right)$. We observe a decrease in $T_{L}$ as separation distance decreases for a pair of rings whose axial activity approaches each other. For rings with outwardmoving axial activity, the rings exhibit a drastic extension in $T_{L}$ within a range of separation distances. In both cases, we observe a transition from attractive to repulsive behavior at a critical distance, indicating the presence of a bifurcation. We show that both behaviors can be described in terms of the difference of two Yukawa potentials. We conclude that ring interactions may be quantifiable in terms of lifetime and velocity and present unexpected behaviors associated with competing interaction and decay mechanisms.

\subsection{Introduction}

Spiral waves have been observed in a wide variety of media, including chemical [1], physical [2,3], and biological settings [4,5]. Recently, attention has been directed towards the existence of scroll waves, the three-dimensional analogue of spiral waves, which rotate around a line defect known as a filament. Filament-filament interactions are of considerable importance in many fields, and have as-yet unresolved dynamics. It is believed that the initiation and interaction of filaments in the excitable substrate of cardiac tissue underlies ventricular tachycardias and arrhythmias [6,7]. However, the presence of cardiac filaments must be either inferred from epicardial [6-9] or transmural mapping using voltage-sensitive dyes or electrodes $[10,11]$. In general, filaments are most often directly observed experimentally in the context of the Belousov-Zhabotinsky $(\mathrm{BZ})$ reaction $[12,13]$.

Recently, quatrefoil reentry $(\mathrm{QR})$ has been shown as a viable means of reproducing multiple (in this case, four) surface singularities and hence two U-shaped (semicircular) filaments in a controlled, repeatable fashion [14,15]. QR follows repeated stimuli applied at a 
single site, and has been used to demonstrate the importance of unequal bidomain anisotropies in cardiac electrodynamics. Previous work has examined 2-D phase singularity interaction dynamics in QR [16], and demonstrates that the interaction dynamics of the singularities in the experimental preparation were more complex than could be adequately explained by the existing numerical models. In this study, we replicate the experimentally observed QR filament configuration using a simulated pair of adjacent circular filaments (scroll rings) oriented along their symmetry axes with varying initial radii and separation distances. By symmetry, the dynamics of semicircular filaments in an excitable half-domain are identical to a circular filament in a whole domain.

\subsection{Methods}

In order to study the interaction effects of paired scroll rings, it is desirable to utilize an excitable system for which a single insolated scroll ring experiences a minimum of translational movement. In this way, we can then attribute any drifting motion to the effects of interaction. It has been mathematically shown that in the case of an untwisted scroll ring with equal diffusion coefficients (such as the Belousov-Zhabotinsky (BZ) reaction) for which the curvature is not too great, the ring shrinks without drifting along the symmetry axis according to the equation [17]

$$
\frac{d\left(R^{2}\right)}{d t}=-2 D,
$$

where $R$ is the radius, and the $D$ is the diffusion coefficient.

We use a two-variable model of the BZ reaction [18] using the Field-Koros-Noyes formulation $[19,20]$

$$
\begin{gathered}
\frac{d v}{d \tau}=\frac{1}{\varepsilon}\left[v(1-v)-\left(2 q \alpha \frac{w}{1-w}+\beta\right) \frac{v-\mu}{v+\mu}\right]+\nabla^{2} v \\
\frac{d w}{d t}=v-\alpha \frac{w}{1-w}+\delta \nabla^{2} w
\end{gathered}
$$

where $v$ is the bromous acid concentration, $w$ is the relative ferroin concentration, and $\delta=D_{w} / D_{v}$ is the ratio of the diffusion coefficients of the two variables, taken to be equal to 1 in this case. The numerical values of the model parameters were identical with those used in [21].

The calculations were performed in a three-dimensional domain using a cylindrical coordinate system $(z, \rho, \theta)$. Since the rings are axisymmetric, the results are independent of $\theta$ and 
therefore, all calculations may be performed on the $(z, \rho)$ plane only. The diffusion coefficient was $2 \times 10^{-5} \mathrm{~cm}^{2} / \mathrm{s}$. To solve Eqs. (6.2) and (6.3), we used an explicit Euler scheme on a regular grid of $240 \times 240$ nodes, with a spatial discretization of $0.04 \mathrm{~mm}$ and a temporal discretization of $0.1 \mathrm{~s}$, and zero-flux boundary conditions.

Figure 6.1 illustrates the initial conditions used to simulate the two modes of quatrefoil reentry, termed cathodal and anodal break, in which rings of opposite chirality face or oppose each other; these terms are used for the purpose of nomenclature only and are not meant to imply that the physiological circumstances have been reproduced. A scroll ring was initiated by setting $v$ and $w$ to appropriate values to produce the needed critical point. For anodal break, the orientation of the refractory area is reversed to produce scroll wave which rotate in the opposite direction. At this point, the trajectory of the scroll ring in the $(z, \rho)$ plane was computed until the ring annihilated. In this scheme, the filament is therefore depicted as a singularity; the location of the singularity was determined using a topological charge method shown previously [16].

\subsection{Results and Discussion}

The results of these simulations are shown in Figs. 6.2 and 6.3, where $T_{L}$ is shown as a function of the initial ring radius, $R_{0}$ and the inter-ring separation, $Z_{0}$, in (A) and the initial trajectories in $(\rho, z)$ are shown in $(\mathrm{B})$. Values of $\left(Z_{0}, R_{0}\right)$ were sampled at intervals of $(0.32,0.64)$ $\mathrm{mm}$; in regions where changes in $T_{L}$ required more detail, values were sampled at $(0.08,0.32)$ $\mathrm{mm}$. We also show the initial velocity of the large radius scroll rings in the $z$-direction in Figs. $6.2 \mathrm{C}$ and $6.3 \mathrm{C}$, created by generated cubic spline fits to the $(z, \rho)$ trajectories. Not unexpectedly, we observed that scroll rings for which $Z_{0}$ was sufficiently large behaved as though they were independent of one another for both configurations. For $Z_{0}>4 \mathrm{~mm}$, the values of $R_{0}{ }^{2} / \mathrm{T}_{L}$ fit the quantity $2 D$ to within $5 \%$, although for values of $R_{0}$ less than $2.56 \mathrm{~mm}$, the analytical solution of Eq. (6.1) fails due to the increased curvature of the ring with smaller radii [17].

For cathodal break, as $Z_{0}$ is further decreased below $4 \mathrm{~mm}, T_{L}$ decreases rapidly with a $\left|\mathrm{d}\left(T_{0}\right) / \mathrm{d}\left(Z_{0}\right)_{\max }\right| \approx 2.23 \mathrm{~mm}$ for large $R_{0}$ (Fig. 6.2A). As can be seen in Fig. 6.2C, the velocity in the $z$-direction increases with $Z_{0}$ and then subsequently falls off for large $Z_{0}$. Note the presence of a zero-crossing for $Z_{O(c r i t)} \approx 2.23 \mathrm{~mm}$ indicating a transition in behavior, from repulsive to 

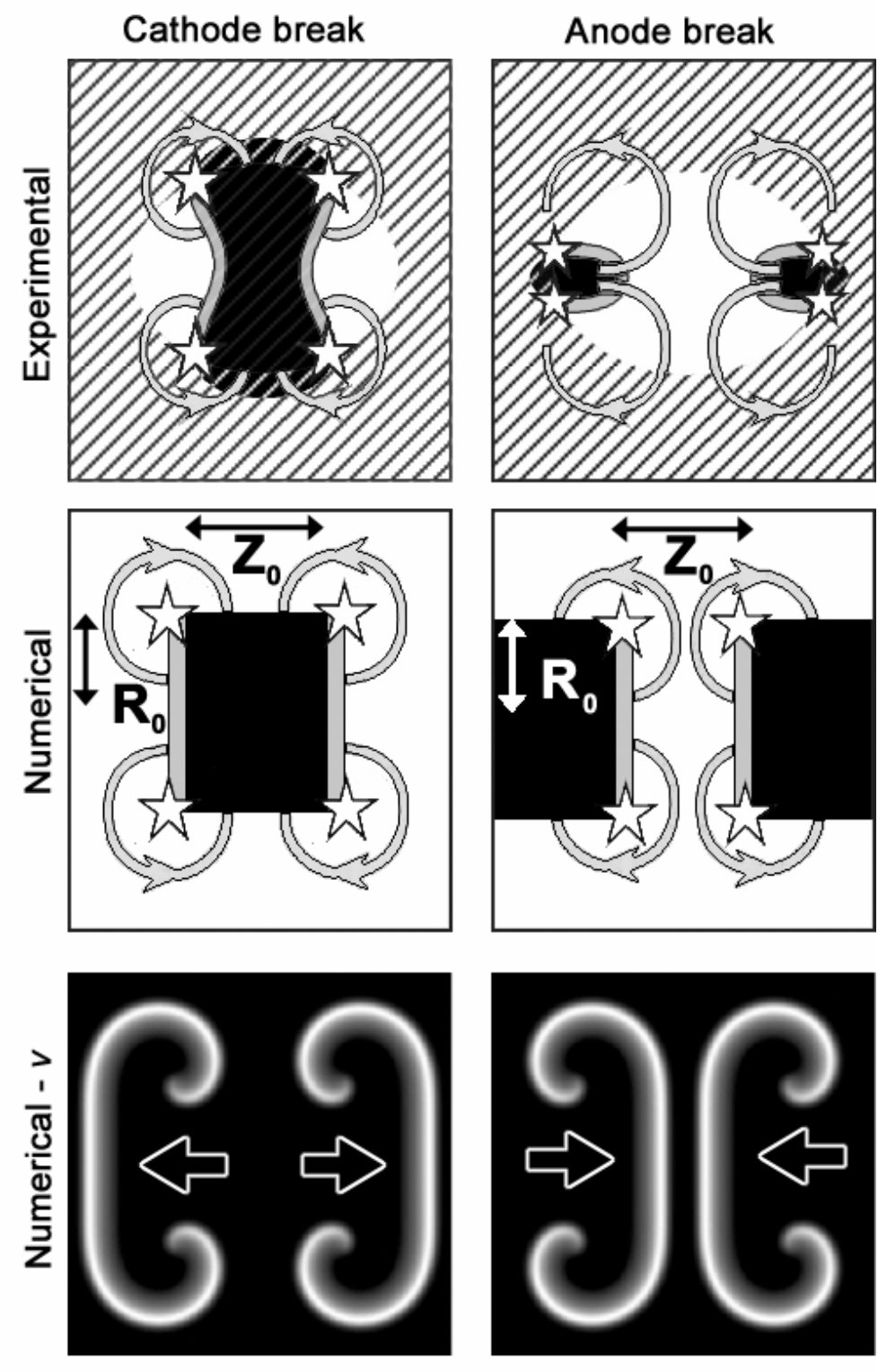

Figure 6.1: Quatrefoil reentry produced by cathodal break (left column) and anodal break (right column). Top row depicts the experimental configuration. Black regions are tissue stimulated by cathodal excitation, gray border is initial wavefront at edge of excited tissue, hatched regions are refractory tissue, white regions are unexecited/hyperpolarized tissue, stars show location of phase singularities). Middle row illustrates initial numerical approximation of the experimental configuration. Black (refractory) is $(v, w)=(0,0.9)$, gray border (excited) is $(v, w)=(0.8,0.8)$, white (unexecited) is $(v, w)=(0,0.8)$. Bottom row depicts the spatial distribution of the variable $v$ after $160 \mathrm{~s}$. The arrows show the direction of the motion of the wave front as it passes through the plane of the ring. 
attractive. Upon examination of the filament trajectories in the $(\mathrm{z}, \rho)$ plane, we observed that between $Z_{0}=2.16-2.32 \mathrm{~mm}$ for large $R_{0}$, there exists a shift in annihilation behavior (from mutual ring collision to individual ring collapse).

For anodal break, as $Z_{0}$ is decreased below $4 \mathrm{~mm}, T_{L}$ actually increases with $Z_{0}$, reaching a maximum of $1.4 \times 10^{5} \mathrm{~s}$ at $Z_{0}=2.4 \mathrm{~mm}$, after which $T_{L}$ falls off dramatically as $Z_{0}$ is further decreased. Much as in cathodal break, the velocity in the $Z$-direction increases with $Z_{0}$ and then subsequently falls off for large $Z_{0}$, with a zero-crossing at $Z_{0 \text { (crit) }} \approx 2.42 \mathrm{~mm}$ (Fig. 6.3C). Examination of the trajectories in the $(z, \rho)$ plane shows that for $Z_{0}<2.4 \mathrm{~mm}$, the rings exhibit attractive behavior and annihilate by collision for large $R_{0}$; furthermore, in this range of $Z_{0}$ for $R_{0}$ $>4.12 \mathrm{~mm}$, the rings expanded as they attract one another. For $Z_{0}>2.4 \mathrm{~mm}$, the rings annihilate by collapse.

The subject of theoretical or numerically simulated 2-D spiral wave interaction has been widely treated in the literature. However, due to the analytic intractability of the problem, similar progress in theoretical filament interaction dynamics is only recently forthcoming. Much of the literature deals with topological concerns [22], self-interaction or stability [17,21,23,24], or motion of a single filament [25-27]; such experiments have been performed mostly with the complex Ginzberg-Landau equations. In general, filament studies are concerned with determining the factors behind filament instability leading to breakup as opposed to the dynamics once breakup occurs and multiple filaments are present.

The lifetime of a vortex has been used as a heuristic previously in the case of a spiral pair in which it was found that spirals placed at a certain initial distance apart, $Z_{0(c r i t)}$, fell into one of

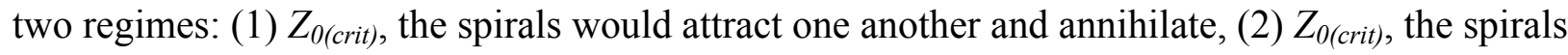
would remain stable infinitely [28]. In much the same way, we see the same regimes in our data, (1) mutual annihilation and (2) collapse via shrinkage, the positive filament tension precluding infinite stability. Furthermore, in [29], an unstable solution of the complex Ginzburg-Landau equations for a single pair of vortices is found to correspond to a change of vortex interaction behavior; the transition across a critical distance changes attractive into repulsive behavior. As Figs. $6.2 \mathrm{C}$ and $6.3 \mathrm{C}$ indicates, this bifurcation is also observed in our data.

A persistent issue in the examination of vortex dynamics is the determination of the parameters involved in modulating the excitable medium which governs close- and long-range 

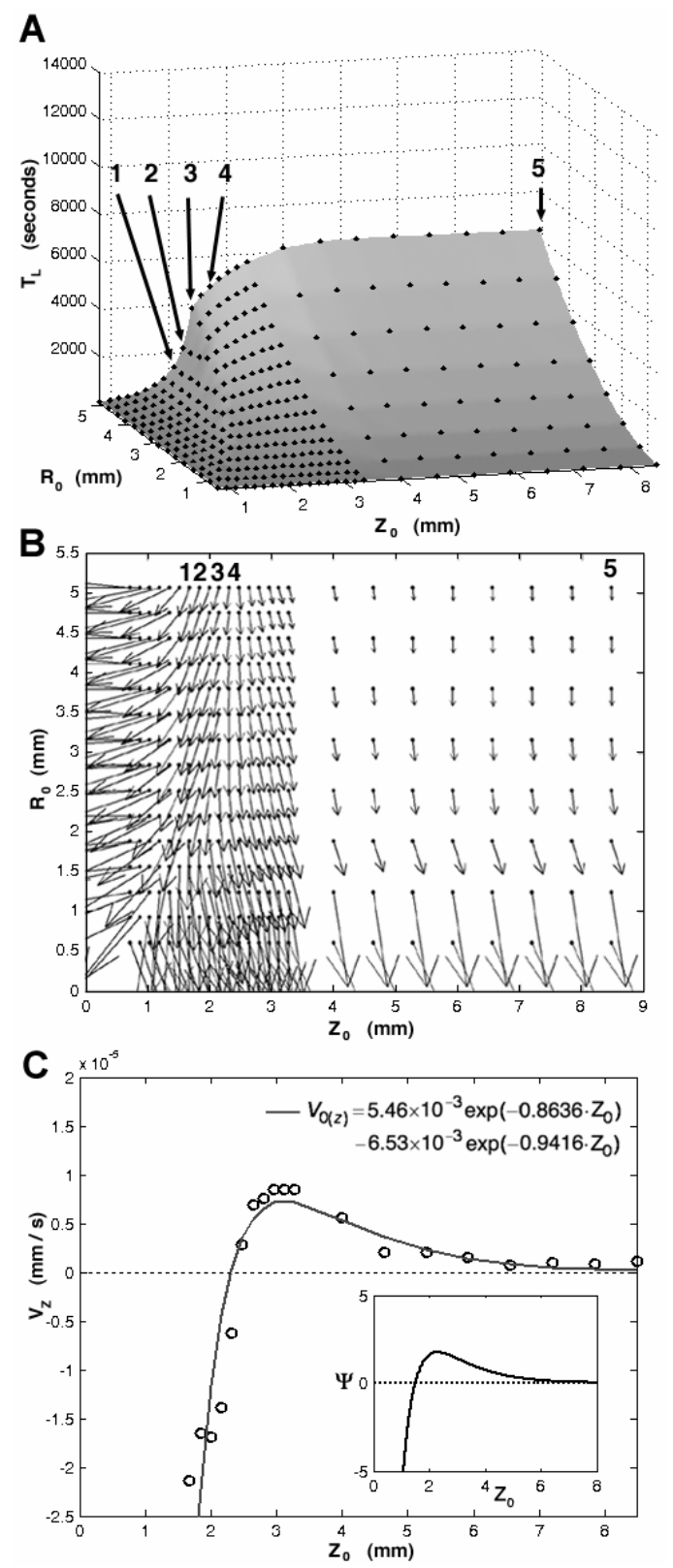

Figure 6.2: (A) Scroll-ring lifetime $T_{L}$ as a function of $\left(R_{0}, Z_{0}\right)$ in cathodal break simulation. Each point is a single simulation for a given $\left(R_{0}, Z_{0}\right)$. Points $1-4$ refer to $\left(R_{0}, Z_{0}\right)=(5.12,2.08)$, $(5.12,2.24),(5.12,2.4),(5.12,2.72)$, and $(5.12,8.48)$, respectively. (B) Initial trajectories in the $(\rho, z)$ plane. (C) Relationship between the initial separation distance $\left(Z_{0}\right)$ and initial scroll rings velocity in the $z$-direction $\left(V_{O(z)}\right)$. The curve represents a fitting of the numerical data with a difference of Yukawa potentials. Graph of $\psi\left(Z_{0}\right)$ shown as inset. 

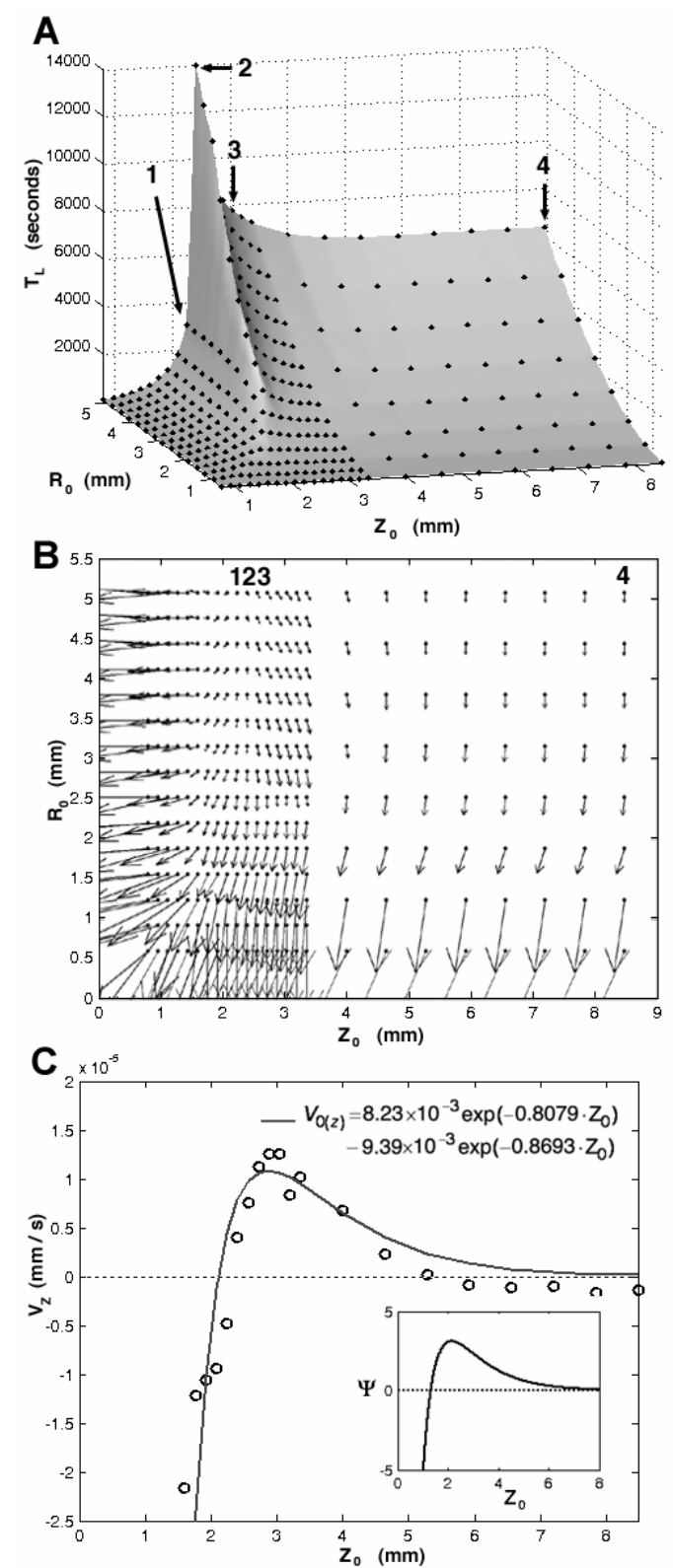

Figure 6.3: (A) Scroll-ring lifetime $T_{L}$ as a function of $\left(R_{0}, Z_{0}\right)$ in anodal break simulation. Each point is a single simulation for a given $\left(R_{0}, Z_{0}\right)$. Points $1-3$ refer to $\left(R_{0}, Z_{0}\right)=(5.12,2.24)$,

$(5.12,2.4),(5.12,2.72)$, and $(5.12,8.48)$, respectively. (B) Initial trajectories in the $(\rho, z)$ plane. (C) Relationship between the initial separation distance $\left(Z_{0}\right)$ and initial scroll rings velocity in the $z$ direction $\left(V_{0(z)}\right)$. The curve represents a fitting of the numerical data with a difference of Yukawa potentials. Graph of $\psi\left(Z_{0}\right)$ shown as inset. 
interactions [30]. It has been shown that the dynamics of vortex systems are analogous to that of a collection of charged particles [31], and hence the problem of vortex-vortex interaction may be analyzed as a particle-field problem. Determination of the inter-vortex distance mediated by underlying potential and the associated force has been extensively studied analytically in the Ginzburg-Landau equation [32]. Approaching the issue of paired scroll ring behavior by reducing the problem to the dynamics of singular filaments allows us to ascertain whether we can determine a filament-filament interaction potential. Here we assume the filament may be treated as a point "mass" within a cylindrical coordinate system; by examining behavior for the largest $R_{0}$ studied ( $R_{0}=5.08 \mathrm{~mm}$, in this case), the filaments all start with the same (unknown) effective "mass". Because these filaments appear to be moving dissipatively rather than ballistically, the initial velocity in the $Z$ direction $V_{0(z)}$ alone can be used as a measure of the force on the filament and hence the interaction potential, $\psi\left(\mathrm{Z}_{0}\right)$. We have found that the dependence of the initial velocity, and hence force, on the initial separation $Z_{0}$ is readily fitted by the difference of two Yukawa potentials [33] of the form

$$
V_{0}\left(Z_{0}\right)=C_{1} \frac{e^{-C_{2} Z_{0}}}{Z_{0}}-C_{3} \frac{e^{-C_{4} Z_{0}}}{Z_{0}}
$$

that describes both the attractive and repulsive behaviors, with the fitted parameter values given in Figs. 6.2C and 6.3C. Equation (6.4) can be integrated trivially to convert force to $\psi\left(\mathrm{Z}_{0}\right)$, where we assume that $\psi(\infty)=0$ (graphs of $\psi\left(\mathrm{Z}_{0}\right)$ are shown as insets).

Mathematically, the configuration of the two scroll rings with axial symmetry is equivalent to a single spiral wave with a reflecting (Neumann) boundary condition normal to the symmetry axis. It is worth noting that in this scenario, the bifurcation in behavior occurs within the space of one spiral wavelength $(\lambda \sim 1.7 \mathrm{~mm})$. In experiments observing BZ spiral drift along a boundary, it is observed that the direction of drift corresponds to the spiral chirality [34]. This explains the direction of drift once the curvature of the independently rotating ring becomes sufficiently great towards the end of the ring's life (note the direction of the vectors for $R_{0}<1$ $\mathrm{mm}$ in Figs. 6.2B and 6.3B). However, it also explains the increased lifetime of the anodal break ring as the initial separation approaches $Z_{0(c r i t)}$ since the interaction of the ring with the "boundary" tends to pull the ring in the $+z$ direction, offsetting the collapse due to filament tension. By the same token, $Z_{0(\text { crit })}$ in cathodal break is close to that of anodal break (2.23 vs. 2.42 
$\mathrm{mm})$; in this case, "boundary"-induced drift cooperates with the filament tension, and hastens ring collapse.

\subsection{Limitations}

As noted earlier, this is a very generalized approximation to the phenomena of anodal and cathodal break excitation in cardiac tissue. In this study, we have used a chemical oscillatory system rather than an ionic active membrane model due to the BZ reaction's well-defined behavior. We have imposed symmetry constraints for the sake of simplicity; a more extensive study in the absence of such constraints, especially without the reflecting boundary condition, would merit future investigation. Bound spiral pairs may be obtained for singly-diffusive systems (of which cardiac models are a subset) [35], in contrast to the doubly-diffusive BZ model in which spirals pairs tend to be repulsive in the presence of any symmetry-breaking between the spirals [36]. Also, we have ignored such structural properties that would present in cardiac tissue such as anisotropic propagation, fiber rotation with depth, and curvature of the extended tissue as would be found in ventricular myocardium. In actuality, the motion of spiral waves on the cardiac epicardium tends to be characterized by meandering upon the surface [3739], due to geometric effects of the filament's curvature as well as the presence of a heterogeneous excitable medium. However, this present study provides some clues as to the behavior of the filament in the relative absence of additional perturbations.

\subsection{Acknowledgments}

We would like to acknowledge useful conversations with R. Aliev and N. Trayanova and comments on the manuscript by D. Ernst. This work was supported in part by NIH grant R01HL58241. The work of M.-A.B. was partially supported by a UNCF-Merck fellowship.

\subsection{References}

[1] Chemical waves and patterns., Kapral, R. and Showalter, K. (eds.) Dordrecht: Kluwer Academic Publishers, 1995.

[2] Jakubith, S., Rotermund, H. H., Engel, W., von Oertzen, A., and Ertl, G., "Spatiotemporal concentration patterns in a surface reaction: Propagating and standing waves, rotating spirals, and turbulence." Phys Rev Lett, vol. 65, no. 24, pp. 3013-3016, 1990. 
[3] Törnkvist, O. and Schröder, E., "Vortex dynamics in dissipative systems." Phys Rev Lett, vol. 78, no. 10, pp. 1908-1911, 1997.

[4] Levine, H., Aranson, I., Tsimring, L., and Truong, T. V., "Positive genetic feedback governs cAMP spiral wave formation in Dictyostelium." Proc Natl Acad Sci USA, vol. 93, no. 13, pp. 6382-6386, 1996.

[5] Gray, R. A. and Jalife, J., "Spiral waves and the heart." Int J Bifurc Chaos, vol. 6, no. 3, pp. 415-435, 1996.

[6] Gray, R. A., Jalife, J., Panfilov, A. V., Baxter, W. T., Cabo, C., Davidenko, J. M., and Pertsov, A. M., "Mechanisms of cardiac fibrillation: Drifting rotors as a mechanism of cardiac fibrillation." Science, vol. 270, no. 5239, pp. 1222-1223, 1995.

[7] Gray, R. A., Jalife, J., Panfilov, A., Baxter, W. T., Cabo, C., Davidenko, J. M., and Pertsov, A. M., "Nonstationary vortexlike reentrant activity as a mechanism of polymorphic ventricular tachycardia in the isolated rabbit heart." Circulation, vol. 91, no. 9, pp. 2454-2469, 1995.

[8] Efimov, I. R., Sidorov, V., Cheng, Y., and Wollenzier, B., "Evidence of threedimensional scroll waves with ribbon-shaped filament as a mechanism of ventricular tachycardia in the isolated rabbit heart." J Cardiovasc Electrophysiol, vol. 10, no. 11, pp. 1452-1462, 1999.

[9] Biktashev, V. N., Holden, A. V., Mironov, S. F., Pertsov, A. M., and Zaitsev, A. V., "Three-dimensional aspects of re-entry in experimental and numerical models of ventricular fibrillation." Int J Bifurc Chaos, vol. 9, no. 4, pp. 694-704, 1999.

[10] Chen, P.-S., Wolf, P. D., Dixon, E. G., Danieley, N. D., Frazier, D. W., Smith, W. M., and Ideker, R. E., "Mechanism of ventricular vulnerability to single premature stimuli in open-chest dogs." Circ Res, vol. 62, no. 6, pp. 1191-1209, 2000.

[11] Baxter, W. T., Mironov, S. F., Zaitsev, A. V., Jalife, J., and Pertsov, A. M., "Visualizing excitation waves inside cardiac muscle using transillumination." Biophys J, vol. 80, no. 1, pp. 516-530, 2001.

[12] Vinson, M., Mironov, S., Mulvey, S., and Pertsov, A., "Control of spatial orientation and lifetime of scroll rings in excitable media." Nature, vol. 386, no. 6624, pp. 447-480, 1997.

[13] Mironov, S., Vinson, M., Mulvey, S., and Pertsov, A., "Destabilization of threedimensional rotating chemical waves in an inhomogeneous BZ reaction." J Phys Chem, vol. 100, no. 5, pp. 1975-1983, 1996.

[14] Lin, S.-F., Roth, B. J., and Wikswo, J. P., Jr., "Quatrefoil reentry in myocardium: An optical imaging study of the induction mechanism." J Cardiovasc Electrophysiol, vol. 10, no. 4, pp. 574-586, 1999. 
[15] Roth, B. J., Lin, S.-F., and Wikswo, J. P., Jr., "Unipolar stimulation of cardiac tissue." $J$ Electrocardiol, vol. 31 Suppl pp. 6-12, 1998.

[16] Bray, M.-A., Lin, S.-F., Aliev, R. R., Roth, B. J., and Wikswo, J. P., Jr., "Experimental and theoretical analysis of phase singularity dynamics in cardiac tissue." $J$ Cardiovasc Electrophysiol, vol. 12, no. 6, pp. 716-722, 2001.

[17] Panfilov, A. V., Rudenko, A. N., and Krinskii, V. I., "Vortical rings in three-dimensional active media with diffusion over two components" Biophysics, vol. 31, no. 5, pp. 926$931,1986$.

[18] Zaikin, A. N. and Zhabotinsky, A. M., "Concentration wave propagation in twodimensional liquid-phase self-oscillating system." Nature, vol. 225 pp. 535-537, 1970.

[19] Field, R. J., Noyes, R. M., and Koros, E., "Oscillations in chemical systems II. Thorough analysis of temporal oscillation in bromate-cerium-malonic acid system." J Amer Chem Soc, vol. 94, no. 25, pp. 8649-8664, 1972.

[20] Rovinsky, A. B. and Zhabotinsky, A. M., "Mechanism and mathematical-model of the oscillating bromate-ferroin-bromomalonic acid reaction." J Phys Chem, vol. 88, no. 25, pp. 6081-6084, 1984.

[21] Vinson, M., "Interactions of spiral waves in inhomogeneous excitable media." Physica D, vol. 116, no. 3, pp. 313-324, 1998.

[22] Fiedler, B. and Mantel, R.-M., "Crossover collision of scroll wave filaments." Documenta Mathematica, vol. 5 pp. 695-731, 2000.

[23] Aranson, I. S. and Bishop, A. R., "Instability and stretching of vortex lines in the threedimensional complex Ginzburg-Landau equation." Phys Rev Lett, vol. 79, no. 21, pp. 4174-4177, 1997.

[24] Nam, K., Ott, E., Guzdar, P. N., and Gabbay, M., "Stability of spiral wave vortex filaments with phase twists." Phys Rev E, vol. 58, no. 2, pp. 2580-2585, 1998.

[25] Keener, J. P. and Tyson, J. J., "The dynamics of scroll waves in excitable media." SIAM Review, vol. 34, no. 1, pp. 1-39, 1992.

[26] Gabbay, M., Ott, E., and Guzdar, P. N., "Motion of scroll wave filaments in the complex Ginzburg-Landau equation." Phys Rev Lett, vol. 78, no. 10, pp. 2012-2015, 1997.

[27] Wellner, M., Berenfeld, O., and Pertsov, A. M., "Predicting filament drift in twisted anisotropy." Phys Rev E, vol. 61, no. 2, pp. 1845-1850, 2000.

[28] Panfilov, A. V. and Vasiev, B. N., "Vortex initiation in a heterogeneous excitable medium." Physica D, vol. 49, no. 1-2, pp. 107-113, 1991. 
[29] Elphick, C. and Meron, E., "Dynamics of phase singularities in 2-dimensional oscillatingsystems." Physica D, vol. 53, no. 2-4, pp. 385-399, 1991.

[30] Wu, X.-G., Chee, M.-N., and Kapral, R., "Vortex dynamics in oscillatory chemical systems." Chaos, vol. 1, no. 4, pp. 421-434, 1991.

[31] Minnhagen, P., "The two-dimensional Coulomb gas, vortex unbinding, and superfluidsuperconducting films." Rev Mod Phys, vol. 59, no. 4, pp. 1001-1066, 1987.

[32] Bodenschatz, E., Pesch, W., and Kramer, L., "Structure and dynamics of dislocations in an anisotropic pattern-forming systems." Physica D, vol. 32, no. 1, pp. 135-145, 1988.

[33] Yukawa, H., "On the interaction of elementary particles." Proc Phys-Math Soc Japan, vol. 17 pp. 48-57, 1935.

[34] Gómez-Gesteira, M., Muñuzuri, A. P., Pérez-Muñuzuri, V., and Pérez-Villar, V., "Boundary-imposed spiral drift." Phys Rev E, vol. 53, no. 5, pp. 5480-5483, 1996.

[35] Ermakova, E. A., Pertsov, A. M., and Shnol, E. E., "On the interaction of vortices in twodimensional active media." Physica D, vol. 40, no. 2, pp. 185-195, 1989.

[36] Brandstadter, H., Braune, M., Schebesch, I., and Engel, H., "Experimental study of the dynamics of spiral pairs in light-sensitive Belousov-Zhabotinskii media using an open-gel reactor." Chem Phys Lett, vol. 323, no. 1-2, pp. 145-154, 2000.

[37] Roth, B. J., "Meandering of spiral waves in anisotropic cardiac tissue." Physica D, vol. 150, no. 1-2, pp. 127-136, 2001.

[38] Kim, D. T., Kwan, Y., Lee, J. J., Ikeda, T., Uchida, T., Kamjoo, K., Kim, Y.-H., Ong, J. J. C., Athill, C. A., Wu, T.-J., Czer, L., Karagueuzian, H. S., and Chen, P.-S., "Patterns of spiral tip motion in cardiac tissues." Chaos, vol. 8, no. 1, pp. 137-148, 1998.

[39] Pertsov, A. M., Davidenko, J. M., Salomonsz, R., Baxter, W. T., and Jalife, J., "Spiral waves of excitation underlie reentrant activity in isolated cardiac muscle." Circ Res, vol. 72, no. 3, pp. 631-650, 1993. 


\title{
CHAPTER VII
}

\section{EXAmination Of Optical Depth EfFects On Fluorescent Imaging Of CARDiac Propagation}

\author{
Mark-Anthony Bray ${ }^{1}$ and John P. Wikswo ${ }^{1,2,3}$ \\ ${ }^{1}$ Department of Biomedical Engineering, \\ ${ }^{2}$ Department of Molecular Physiology and Biophysics \\ ${ }^{3}$ Department of Physics and Astronomy, \\ Vanderbilt University \\ Nashville, TN 37235
}

This manuscript has been submitted for publication in Biophysical Journal. 


\subsection{Abstract}

Optical mapping with voltage-sensitive dyes provides a high-resolution technique to observe cardiac electrodynamic behavior. While most studies assume that the fluorescent signal is emitted from the surface layer of cells, the effects of signal attenuation with depth on signal interpretation are still unclear. This simulation study examines the effects of a depth-weighted signal on epicardial activation patterns and filament localization. We simulated filament behavior using a detailed cardiac model, and compared the signal obtained from the top (epicardial) layer of the spatial domain with the calculated weighted signal. General observations included a prolongation of the action upstroke duration, early upstroke initiation, and reduction in signal amplitude in the weighted signal. A shallow filament was found to produce a dual-humped action potential morphology consistent with previously reported observations. Simulated scroll wave breakup exhibited effects such as the false appearance of graded potentials, apparent supramaximal conduction velocities, and a spatially blurred signal with the local amplitude dependent upon the immediate sub-epicardial activity; the combination of these effects produced a corresponding change in the accuracy of filament localization. Our results indicate that the depth-dependent optical signal has significant consequences on the interpretation of epicardial activation dynamics.

\subsection{Introduction}

Over the past thirty years, optical mapping has been shown to be a powerful tool for discerning cardiac activation patterns, in both isolated single cell [1,2] and whole heart preparations $[3,4]$. In such experimental applications, the cell or tissue is stained with a voltagesensitive fluorescent dye, that binds to the cellular membranes and transduces transmembrane potential chnages into fluorescent signals. Such methods permit a non-contact means of recoding electrical activity with temporal resolution on the order of microseconds [5] and spatial resolutions on the scale of 50-100 $\mu \mathrm{m}$ [6]. The majority of the current optical mapping studies assume that the fluorescent signal is emanating from the surface layer of the cardiac tissue preparation. In this way, the observed dynamic behavior is presumed to be independent of the underlying electrodynamic activity within the myocardium. While this approximation may suffice for some preparations, such as those involving cultured cell layers [7], recently studies 
involving sections of myocardium of more physiologically realistic thickness are questioning the validity of this assumption $[8,9]$.

The question of the origin of the optically recorded signal is particularly relevant in light of more recent literature attempting to make inferences on the underlying electrical activity on the basis of reentrant activity observed on the surface. The spiral waves associated with reentry rotate around topological defects known as phase singularities [10]. It is believed that the phase singularity is the surface manifestation of a line defect known as a filament, which in turn forms the organizing center of a scroll wave, the three-dimensional analogue of a spiral wave. Since the myocardium is a three-dimensional excitable substrate, the additional degree of freedom makes the contribution of filaments more complex to discern, yet no less important to the study of arrhythmogenesis [11]. The extreme technical difficulty of filament observation in situ has made experimental studies of their dynamics limited primarily to examination of oscillatory chemical reactions $[12,13]$. However, recently the depth effects of the optical signal have been used to infer the existence of underlying filaments based upon the epicardial presence of "dual-humped" action potentials $[14,15]$, and others have tailored their imaging technique to pinpoint the location of signals originating from sub-epicardial tissue [16].

In this study, we will examine of the effects of the inclusion of optical averaging with depth in computational simulations of spiral and scroll wave activity. We will begin with a simple, single filament configuration and progress forward to the complex case of the behavior of multiple filaments and the corresponding phase singularity localization during fibrillation. By so doing, our goal is to gain valuable insight in anticipating potential difficulties in the interpretation of experimentally observed epicardial electrical activity in terms of scroll wave dynamics.

\subsection{Methods}

For this study, we use the three-variable model implemented by Fenton and Karma for the purpose of reproducing the reentrant wave behavior of more complex ionic models while remaining computationally tractable for 3-D applications [17]. The equations are

$$
\begin{aligned}
& \partial u / d t=D \nabla^{2} u-J_{f i}(u, v)-J_{s o}(u)-J_{s i}(u, w), \\
& \partial v / d t=H\left(u_{c}-u\right)(1-v) / \tau_{v}^{-}(u)-H\left(u-u_{c}\right) v / \tau_{v}^{+}, \\
& \partial w / d t=H\left(u_{c}-u\right)(1-w) / \tau_{w}^{-}-H\left(u-u_{c}\right) w / \tau_{w}^{+},
\end{aligned}
$$


where $u$ is the dimensionless transmembrane potential, $v$ and $w$ are gating variables responsible for inactivation and reactivation of the currents $J_{f i}, J_{s o}$, and $J_{s i}$, defined by

$$
\begin{aligned}
& J_{f i}(u, v)=-\frac{v}{\tau_{d}} H\left(u-u_{c}\right)(1-u)\left(u-u_{c}\right), \\
& J_{s o}(u)=-\frac{u}{\tau_{o}} H\left(u_{c}-u\right)+\frac{1}{\tau_{r}} H\left(u-u_{c}\right), \\
& J_{s i}(u, w)=-\frac{w}{2 \tau_{s i}}\left(1+\tanh \left[k\left(u-u_{c}^{s i}\right)\right]\right), \\
& \tau_{v}^{-}(u)=H\left(u-u_{v}\right) \tau_{v 1}^{-}+H\left(u_{v}-u\right) \tau_{v 2}^{-},
\end{aligned}
$$

and $H(x)$ is the standard Heavside step function $(H(x)$ evaluates to 0 for $x<0$ and 1 for $x \geq 0)$.

For this paper, we employ the parameters values used to fit the model to the modified Luo-Rudy (MLR) and modified Beeler-Reuter (MBR) restitution curves; These values are provided in [17], so we omit them here. $\tilde{D}$ is the diffusion tensor defined as

$$
\tilde{D}=\left[\begin{array}{ccc}
D_{x x} & D_{x y} & 0 \\
D_{y x} & D_{y y} & 0 \\
0 & 0 & D_{z z}
\end{array}\right]
$$

where the zero-elements reflect the fact that the fibers all lie in the $(x, y)$ plane. The remaining elements are defined as

$$
\begin{gathered}
D_{x x}=D_{\|} \cos ^{2} \theta(z)+D_{\perp} \sin ^{2} \theta(z), \\
D_{y y}=D_{\|} \sin ^{2} \theta(z)+D_{\perp} \cos ^{2} \theta(z), \\
D_{x y}=D_{y x}=\left(D_{\|}-D_{\perp}\right) \cos \theta(z) \sin \theta(z),
\end{gathered}
$$

where $D_{\|}$and $D_{\perp}$ are the diffusion coefficients for propagation parallel and perpendicular to the local fiber orientation, respectively. $\theta(z)$ is the angle between the $x$-axis and the fiber orientation in plane $z$ and is determined by the formula

$$
\theta(z)=-\theta_{\Sigma} / 2+\left(\theta_{\Sigma} / Z\right) z, \quad 0 \leq z \leq Z
$$

where $\theta_{\Sigma}$ is the total amount of fiber rotation from epicardium $(z=0)$ to endocardium $(z=Z)$ as measured from the $x$-axis. The coefficients $D_{\|}$and $D_{\perp}$ are defined as $\sigma /\left(S_{\nu} C_{m}\right)$ where $\sigma_{\|}=1.863$ $\mathrm{mS} / \mathrm{cm}$ and $\sigma_{\perp}=0.186 \mathrm{mS} / \mathrm{cm}$ (in accordance with values estimated by Roth [18]), $S_{v}$ is the surface to volume ratio $\left(3000 \mathrm{~cm}^{-1}\right)$, and $C_{m}$ is the membrane capacitance $\left(1 \mu \mathrm{F} / \mathrm{cm}^{2}\right)$. $\sigma_{z}$ was set equal to $\sigma_{\perp}$. We imposed no-flux (Neumann) boundary conditions, i.e., 


$$
\hat{n} \cdot(\tilde{\sigma} \nabla u)=0
$$

where $\hat{n}$ is the normal vector to the boundaries of the spatial domain. Eq. (7.8) leads to the following equations:

$$
\begin{array}{rr}
\tilde{\sigma}_{x x} \partial_{x} u+\tilde{\sigma}_{x y} \partial_{y} u=0 & \text { for surfaces } x=0 \text { and } x=L_{x}, \\
\tilde{\sigma}_{y x} \partial_{x} u+\tilde{\sigma}_{y y} \partial_{y} u=0 & \text { for surfaces } y=0 \text { and } y=L_{y}, \\
\partial_{z} u=0 & \text { for } z=0 \text { and } z=L_{z} .
\end{array}
$$

The equations in (7.2) are solved explicitly using a finite difference scheme. The spatial discretization steps in all three dimensions were set to $0.133 \mathrm{~mm}$ with a time step of $20 \mu \mathrm{s}$.

With this model, the study was conducted in three stages: (1) a 2-D cross section representative of a single straight intramural filament; (2) examination of a rectangular slab with no fiber rotation, i.e., $\theta_{\Sigma}=0$ and; (3) examination of a rectangular slab with rotational anisotropy with $\theta_{\Sigma}=120^{\circ}$.

The fluorescent signal corresponding to the depth-weighted transmembrane potential is given as

$$
\bar{u}(x, y)=\frac{\int_{0}^{Z} u(x, y, z) w(z) d z}{\int_{0}^{Z} w(z) d z}
$$

where the integral is performed from the epicardium $(z=0)$ to the endocardium $(z=Z)$. w $(z)$ is the epicardial weighting function experimentally determined by Baxter et al. in a transillumination study of sheep right ventricle [19],

$$
w(z)=\left(907 \mathrm{e}^{-z / 0.8}-702 e^{-z / 0.44}\right) e^{-z / 1.34} \quad(z \text { in mm }) .
$$

We use $u_{0}$ to refer to the $u$ values located at the epicardium (the top layer of grid nodes, $z=0$ ) and $\bar{u}$ to refer to the signal derived from the depth-weighted average of $u$.

\subsection{Results}

\subsubsection{Cross-section of intramural filament}

The initial study observed a single intramural filament, located close and parallel to the epicardium. We model this simple case as a two-dimensional cross-section of 

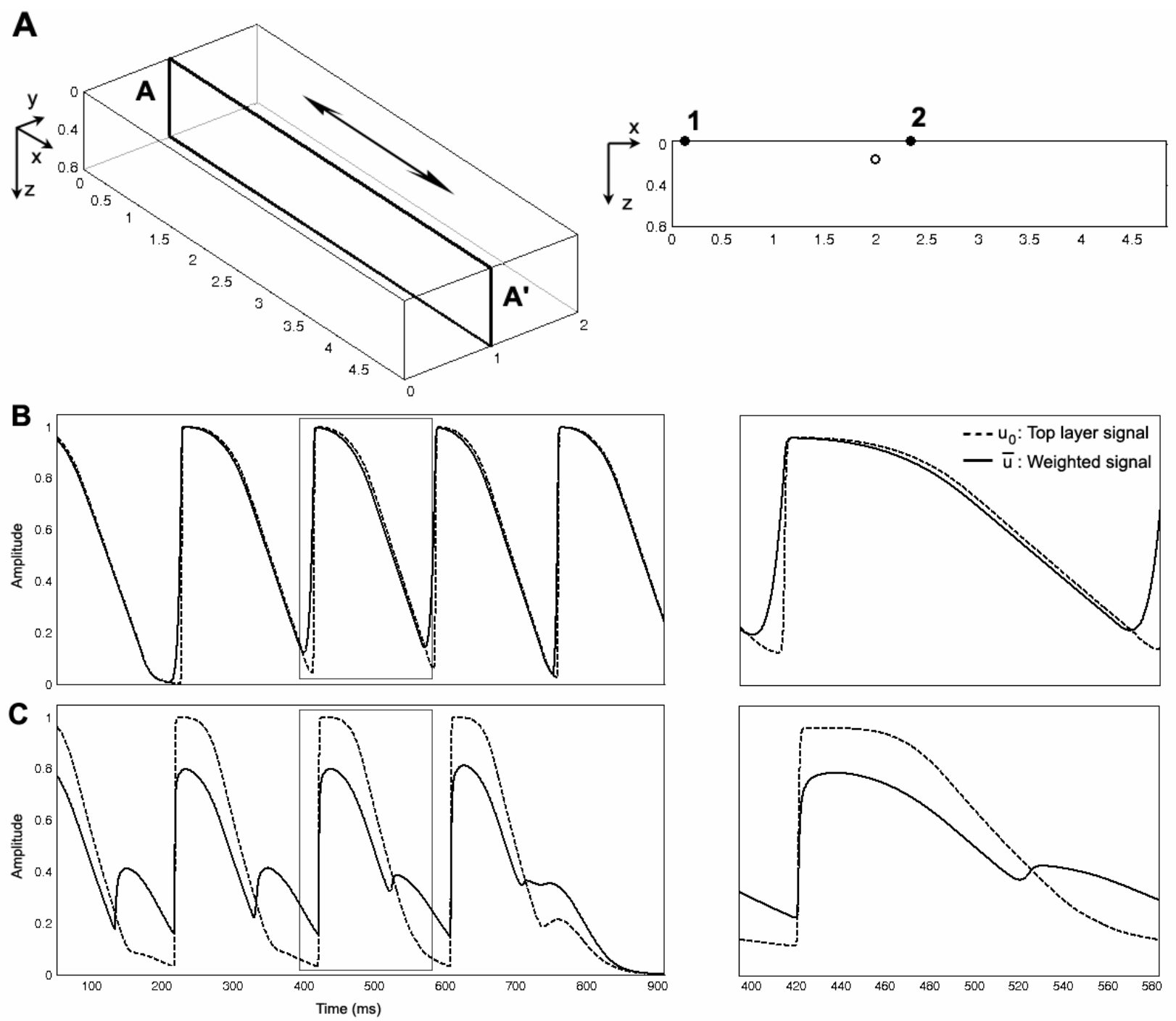

Figure 7.1: (A) The three-dimensional model (left) and the cross-section AA' (right) showing the position of the singularity at $t=0 \mathrm{~ms}$ (open circle) and the two recording locations on the epicardium (1 and 2). Double arrow shows longitudinal fiber direction; spatial units are in $\mathrm{cm}$. Time traces at point (1) are shown in (B); time traces at point (2) are shown in (C). Panels on the right are insets of selected intervals indicated with a box in the panels on left.

tissue in the fiber plane; since the single straight filament is oriented perpendicular to the longitudinal fiber direction, it appears as an isolated singularity in the cross-section with an initial location $(x, z)$ of $(2,0.13) \mathrm{mm}$. For the model, we have chosen to use the parameter values specific to the MLR restitution curve. The reason for this choice is that the observations of Efimov et al. [14] indicated the presence of a long, linear core around which the reentrant wave rotated, a property also shared by the MLR model. For the tissue, we used a $4.8 \mathrm{~cm}$ x $0.8 \mathrm{~cm}$ 
section ( $362 \times 62$ nodes), with the longer dimension corresponding to the longitudinal fiber direction; the geometry is shown in Fig. 7.1A. To localize the phase singularity (the 2-D crosssection of the filament), we use the state-space methodology of determining cardiac phase [20] in conjunction with the topological charge algorithm developed by Bray et al. [21]. We have shown previously that using the $u$ and $v$ variables in the 3 -variable model produces the optimal phase portrait [22]. The origin in $(u(t), v(t))$ state-space was set to $(0.175,0.03)$.

Figure 7.1B compares the $u_{0}$ and $\bar{u}$ signals obtained from a point on the epicardial surface at point $1(0.13,0)$, far from the singularity. We see that while the morphology of $\bar{u}$ is similar to that of $u_{0}$, there are three noticeable differences between the two signals: (1) the upstroke of $\bar{u}$ starts earlier; (2) $\bar{u}$ is greater in amplitude than $u_{0}$ during the upstroke until the peak of both signals; and (3) the repolarization trace of $\bar{u}$ is lower in amplitude than that of $u_{0}$ until the following upstroke. Figure $7.1 \mathrm{C}$ shows $\bar{u}$ and $u_{0}$ at point $2(2.33,0)$, an epicardial point directly above the transmural reentrant core at the closest point of approach of the scroll wave with the epicardial surface. Here we observe that (1) the $\bar{u}$ signal has a dual humped morphology, with the first peak much larger in magnitude that the second; and (2) both peaks of $\bar{u}$ are significantly smaller in amplitude than the single peak of $u_{0}$.

Figure 7.2 depicts $u_{0}$ and $\bar{u}$ in the form of a time-space plot. Time-space plots are a simple means of illustrating the spatiotemporal evolution of the transmembrane potential signal; successive frames of the amplitude of a signal along a line are stacked to form a 2-D plot of activation behavior, with time as the $y$-axis [11,23]. The one-dimensional nature of $u_{0}$ and $\bar{u}$ in this simulation makes it ideal for this form of visualization. In Fig. 7.2A, we see the time course of the $u_{0}$ signal across the length of the tissue. The presence of epicardial breakthroughs, labeled "EB" in the figure, is apparent by the V-shaped patterns of activation in the plot. The corner of the V-pattern of depolarization moves upward with time, indicating that the location of the initial breakthrough is non-stationary. We have used white lines in Fig. 7.2A to indicate the slope of activation branches in the vicinity of the breakthrough; the slope of the line is inversely related to the propagation velocity. Also of note is that the slope of the left activation branch is shallower (and hence, the apparent conduction velocity is faster) than that of the right branch (approximately $74.0 \mathrm{~cm} / \mathrm{s}$ versus $43.5 \mathrm{~cm} / \mathrm{s}$ ). In fact, the conduction velocity of the left branch is faster than the maximum conduction velocity for the model in a 1-D cable where $D$ is set to $D_{\|}$ $(\sim 47 \mathrm{~cm} / \mathrm{s})$. 

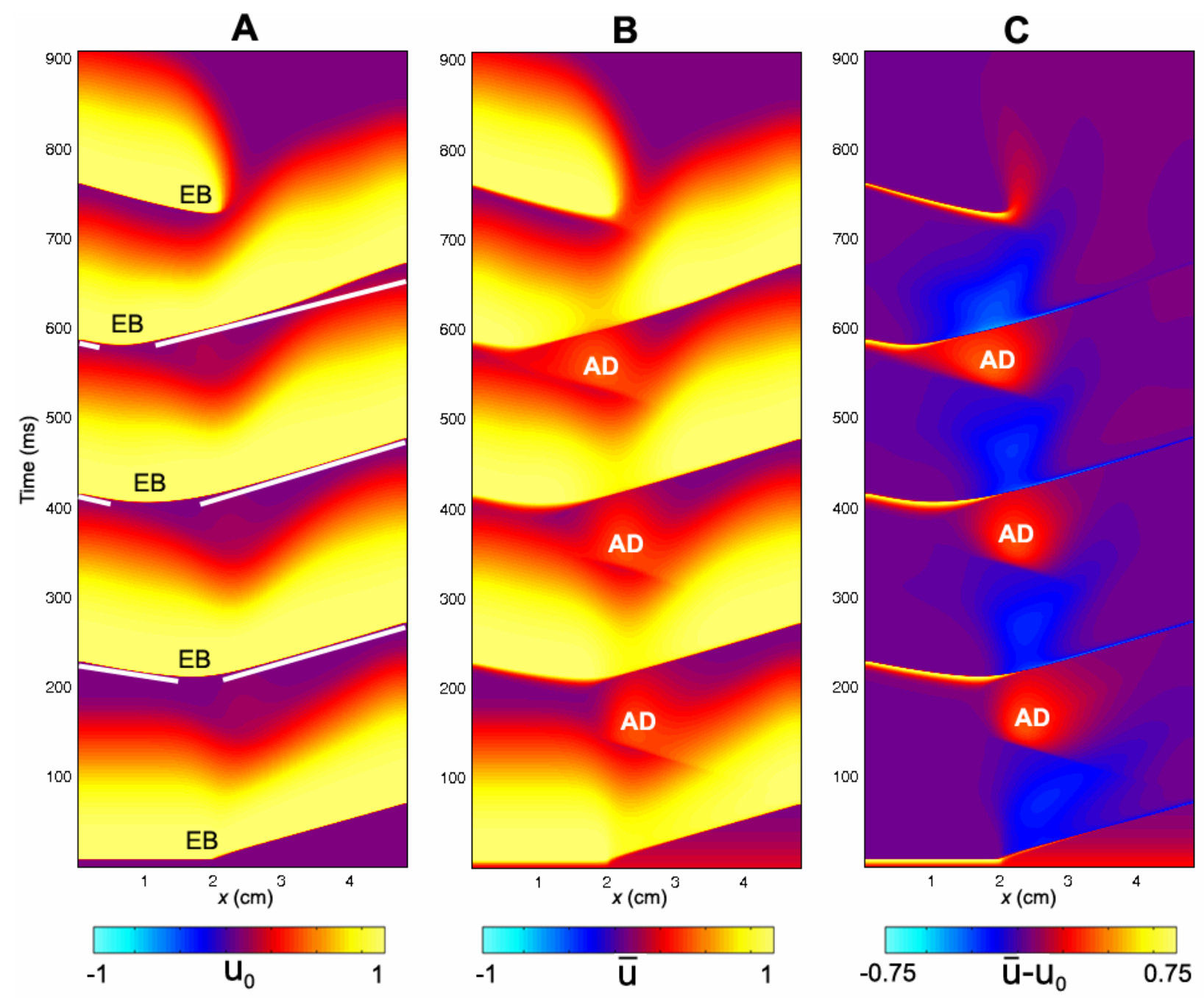

Figure 7.2: Time-space plots of $u$ from the epicardial surface. (A) Top layer of nodes. Solid white lines show slope of activation. (B) Weighted average. (C) Difference between (A) and (B). Epicardial breakthroughs labeled as EB, additional secondary depolarizations labeled as AD.

In Figure 7.2B, illustrating the $\bar{u}$ signal, we clearly see an additional "depolarizations" (AD) in between the epicardial breakthrough patterns, lying in the region roughly between $x=$ 1.5 to $3 \mathrm{~cm}$. This behavior resembles a passive response (as opposed to an action potential) since it is transient and is restricted to a local region of tissue. However, the peak magnitude of the depolarization is on the order of 0.42 , far above the threshold of activation $(u$ is in the range of $[0,1])$. In Fig. $7.2 \mathrm{C}$, we display the result of subtracting the $u_{0}$ from the $\bar{u}$ images, where the orange and yellow hues indicate the areas where $\bar{u}$ is greater than $u_{0}$, purple is close to zero, and the blue hue indicates where $u_{0}$ is greater than $\bar{u}$. We see that the presence of these apparent depolarizations is highlighted by the oval regions of orange. Furthermore, we see adjacent 
A
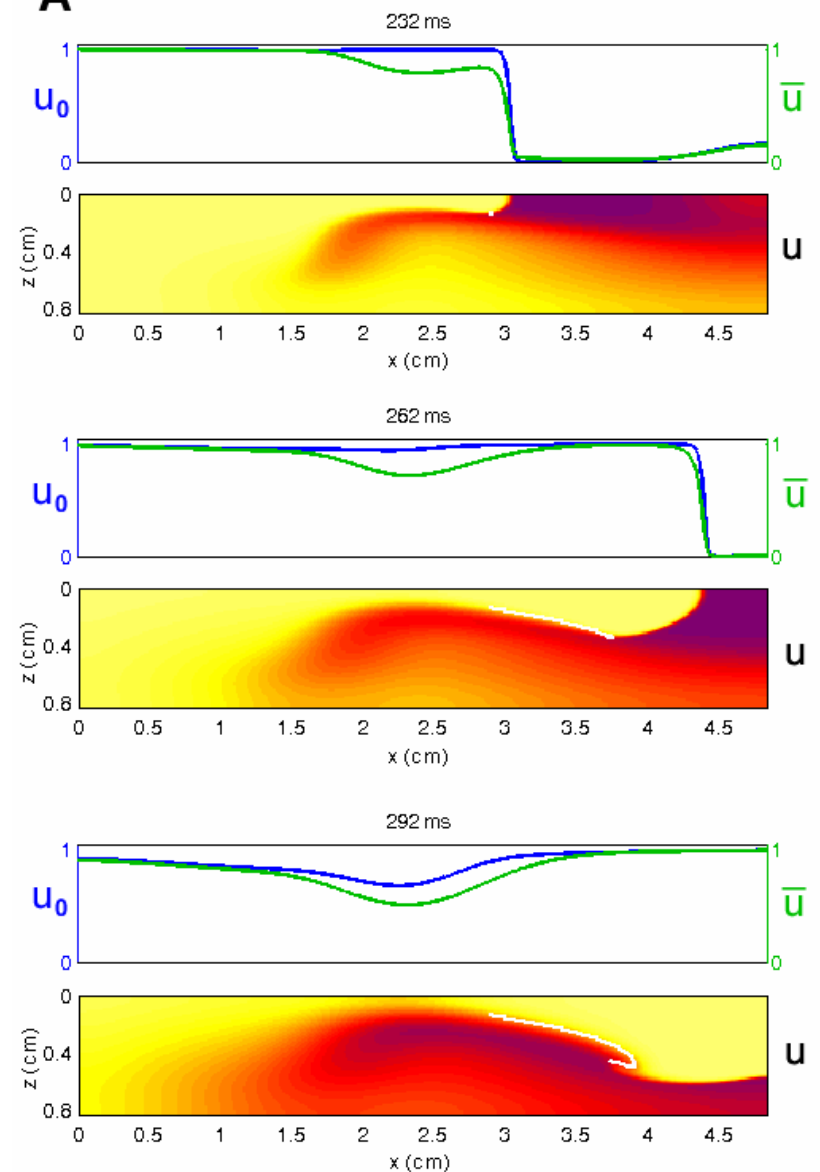

B
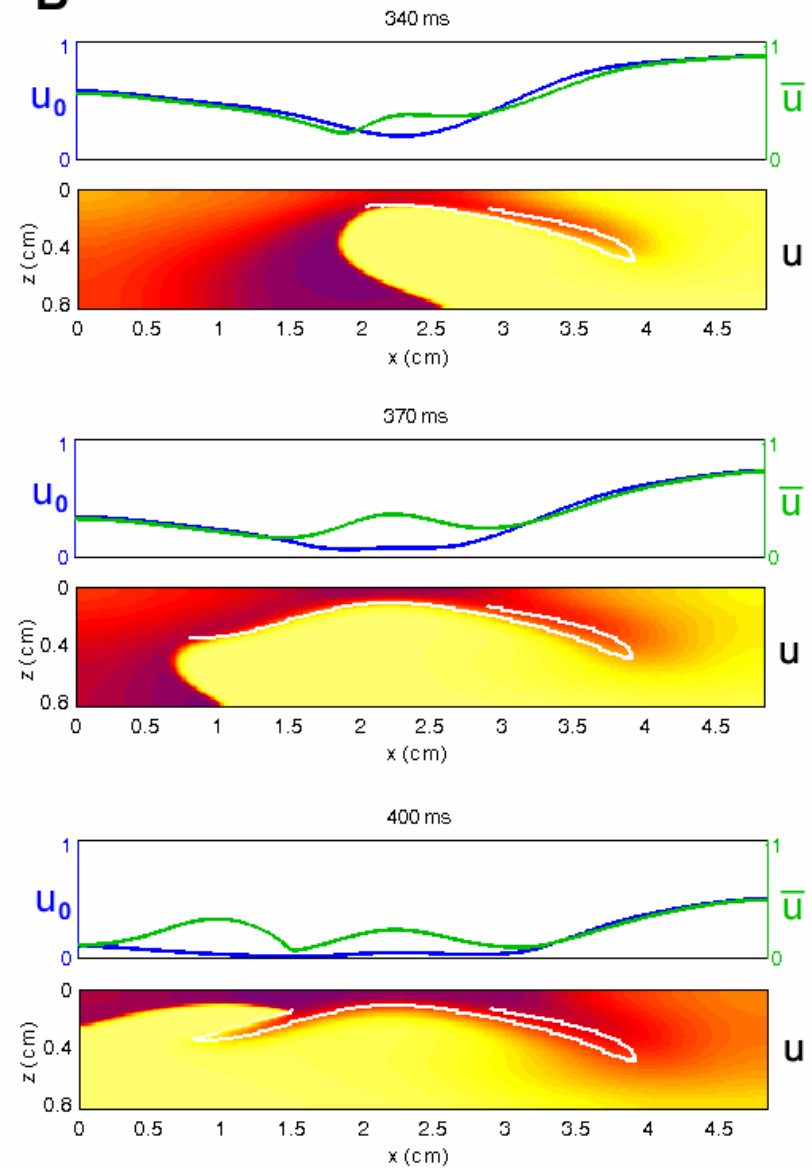

Figure 7.3: Snapshots of the temporal development of the depth-weighted and top layer signals. Top panels: $\bar{u}$ and $u_{0}$ signals as a function of $x$. Bottom panels: Corresponding scroll wave cross-section, with the filament trajectory overlaid as white line. Figure shows wave traversing (A) above and (B) below the core as traced out by the white line.

regions of blue color indicating that in these particular areas, the magnitude of the weighted signal in $\bar{u}$ significantly underestimates the magnitude of the $u_{0}$ signal in the epicardial layer.

The reason for both of these phenomena is shown in Fig. 7.3, where we show the scroll wave cross-section $u(x, z)$ and filament trajectory (bottom panels), along with the corresponding $\bar{u}$ and $u_{0}$ traces as a function of $x$ (top panels); the difference in the two traces over time corresponds to Fig. 7.2C. As mentioned above, reentrant waves in the MLR model possess a linear core trajectory. Hence, in Fig. 7.3A at $t=262 \mathrm{~ms}$, the wave is rotating clockwise and is in the process of completing the upper arc of its trajectory (as shown by the white line). We see that the trajectory has brought the leading edge of the wave near to the epicardial surface; hence, $u_{0}$ is still close to maximally depolarized at the region of closest approach. Meanwhile, we see that as 
the wave completes the turn of its reentrant trajectory $(t=370 \mathrm{~ms})$, the portion of tissue underneath the arc is in the process of repolarization. While the depolarized tissue within the first few epicardial layers plays a substantial role in the magnitude of the weighted signal, the reduced value of $u(x, z)$ in the repolarizing mid-myocardial layers contribute as well. The net effect of the summation over depth at this point in time is that $\bar{u}$ is depressed with respect to $u_{0}$ (see the first peak of the double potential in Fig. 7.1B), with the maximum difference located at the point of closest approach, producing the negative blue regions in Fig. 7.2C.

Likewise, at $t=340 \mathrm{~ms}$ in Fig. 7.3B, the filament is traversing the lower half of the linear arc. Since the closest approach of the filament brings it to within $1.07 \mathrm{~mm}$ of the epicardial surface, those repolarizing surface layers experience a slight passive response, corresponding to a very small positive deflection in $u_{0}$ for those cells. On the other hand, the contribution of the depolarized intramural layers is sufficient to create a significant positive deflection in the weighted $\bar{u}$ signal, as seen in the second peak of the double potential in Fig. 7.1B. Again, the location of the peak corresponds to the local maxima in Fig. 7.2C (orange regions) at that point in time.

Further examination of scroll wave patterns can provide additional details about the relationship between $u_{0}$ and $\bar{u}$. The intramural wavefront approaching the epicardium on the upper left of $u(x, z)$ in Fig. 7.3B at $t=400 \mathrm{~ms}$, produces the earlier upstroke of $\bar{u}$ (in comparison to $u_{0}$ ) as expected, at the point when the wavefront is about $2 \mathrm{~mm}$ from the epicardium, with a clear peak at $x=1.0 \mathrm{~cm}$. This value is in agreement with earlier studies of Baxter et al. [19]. We also observe that $\bar{u}$ is greater than $u_{0}$ only for the duration of the upstroke in the regions where the wavefront is approaching the epicardium. As the wave progress, $\bar{u}$ is lower in magnitude than $u_{0}$ due to the decrease in $u$ with depth during repolarization associated with the decreasing fluorescence contribution from the lower layers. However, in the areas where the wavefront is traveling away from the epicardium, such as in the upper right of $u(x, z)$ in Fig. 7.3A, $t=362 \mathrm{~ms}$, $u_{0}$ remains greater than $\bar{u}$ for the duration of the upstroke, and the two signals follow almost identical time courses for $x \in[0,1.6]$ and $[3.2,4.8] \mathrm{cm}$ during this rotation. Based upon the trajectory of the scroll wave with respect to the epicardium, we can also see the reason for the apparent supra-maximal velocity. The scroll wave approaches the epicardial surface at a shallow oblique angle as can be seen in the upper left corner of the $u$ image at $t=400 \mathrm{~ms}$. This is exhibited in the difference image in Fig. $7.2 \mathrm{C}$ as a yellow ridge along the left branches indicating 

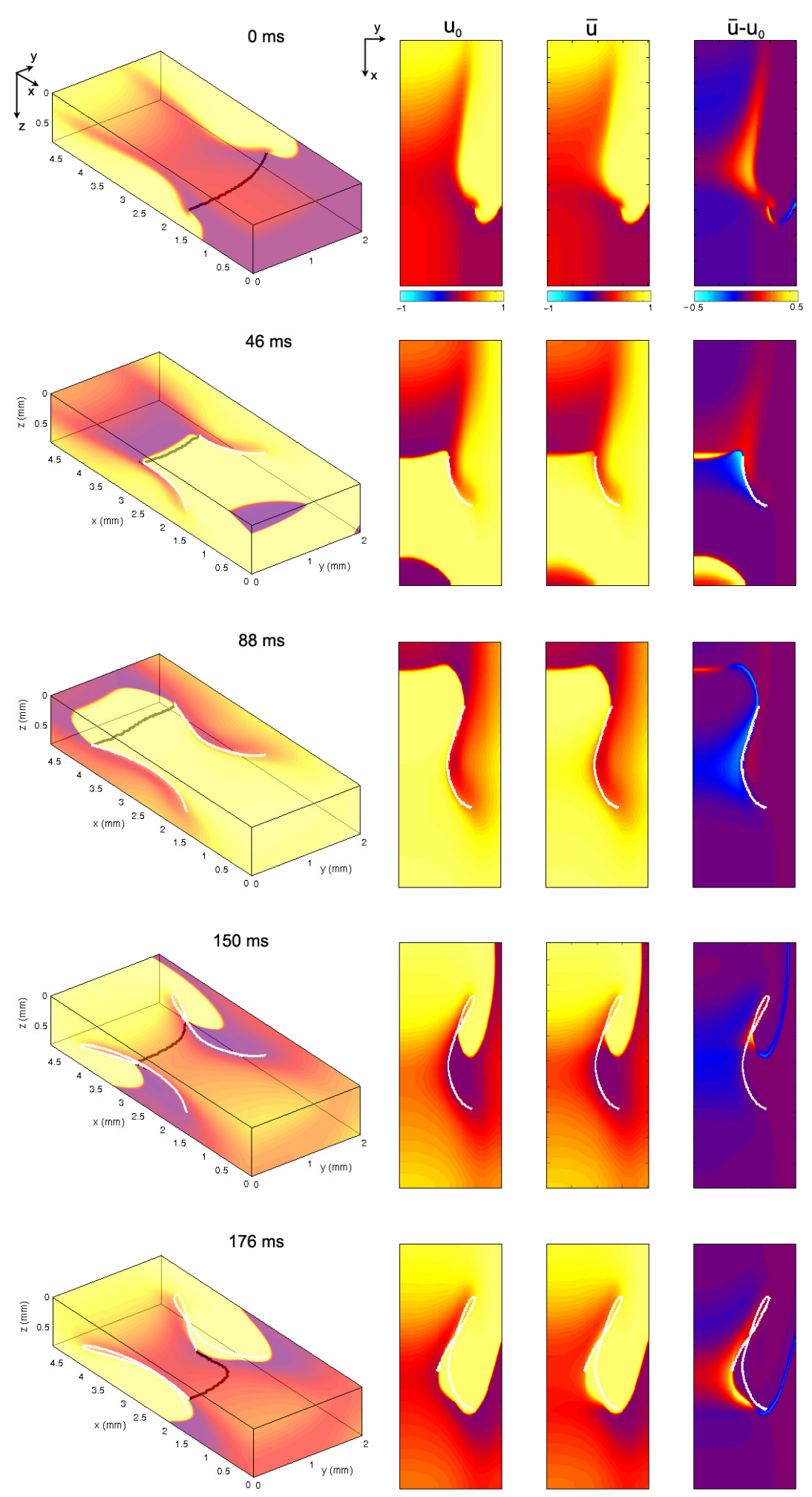

Figure 7.4: Snapshots of the temporal development of the depth-weighted and top layer signals for an elliptical filament. $1^{\text {st }}$ column: 3 -D tissue slab with filament in black. $2^{\text {nd }}$ column: $u_{0} \cdot 3^{\text {rd }}$ column: $\bar{u} \cdot 4^{\text {th }}$ column: $\bar{u}-u_{0}$. Trajectory of the filament on the bounding surfaces is shown in all columns as a white line. 
that $\bar{u}$ increases rapidly while $u_{0}$ remains at a fairly constant value. This situation continues until the actual moment of breakthrough when the underlying wavefront arrives at the epicardium.

\subsubsection{3-D elliptical filament, no fiber rotation}

The second study consisted of our observing a shallow elliptical filament, located close to the epicardium. We model this preparation as a three-dimensional section of tissue. The number of grid points is $362 \times 151 \times 62$, such that the tissue slab has dimensions $4.8 \times 2.0 \times 0.8 \mathrm{~cm}^{3}$, with the longer dimension corresponding to the longitudinal fiber direction. In this case, the filament has one end located on the upper, epicardial surface and the other on a longitudinal face. Again, we use the MLR parameter values for the active kinetic model.

Figure 7.4 illustrates the results from this portion of the study. The first column shows the filament and its trajectory within the tissue slab with $u$ mapped on the bounding surfaces; the orientation of the slab and the fiber direction is the same as that shown in Fig 7.1A. At $t=0 \mathrm{~ms}$, the scroll wave is completing a turn at the end of a line of conduction block. The column depicting $\bar{u}-u_{0}$ indicates that there is a positive difference between $\bar{u}$ and $u_{0}$ at the region where the underlying scroll wave is closest to the epicardium. Furthermore, the magnitude of $\left(\bar{u}-u_{0}\right)$ is greatest at the shallowest portion of the scroll wave, adjacent to the conduction block, but decreases rapidly in the direction of the filament's major axis (i.e., transverse to the fiber direction) due to the short space constant of the weighting function with respect to the increasing filament depth.

At $t=46 \mathrm{~ms}$, the leading edge of the scroll wave is propagating above the area of conduction block. Therefore, we see positive $\left(\bar{u}-u_{0}\right)$ in those regions where the wavefront is close to the epicardium but has not yet broken through, namely at the borders of the breakthrough activation in $u_{0}$. Also, $\left(\bar{u}-u_{0}\right)$ is negative in the region of the leading edge's closest approach to the epicardium, decreasing in magnitude along the major axis of the filament adjacent to the intersection of the conduction block with the epicardium.

The wavefront begins turning a corner at $t=88 \mathrm{~ms}$ and continues the behavior described above. At $t=150 \mathrm{~ms}$, the scroll wave has completed the turn and the region of positive $\left(\bar{u}-u_{0}\right)$ is at the inner edge of the wave adjacent to the tip. In this case, this region does not extend very far along the filament due to the fact that the epicardial activation is still significant and the underlying scroll wave is not yet within the space constant of the weighting function. At $t=176$ 
A
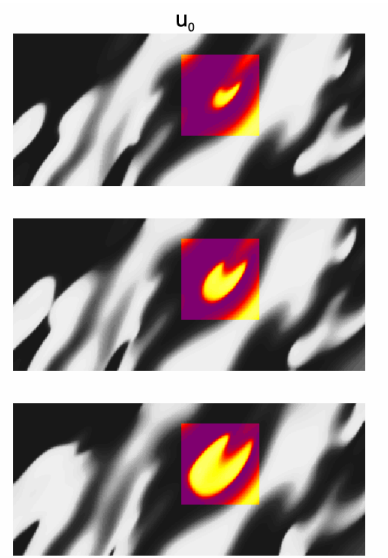

C
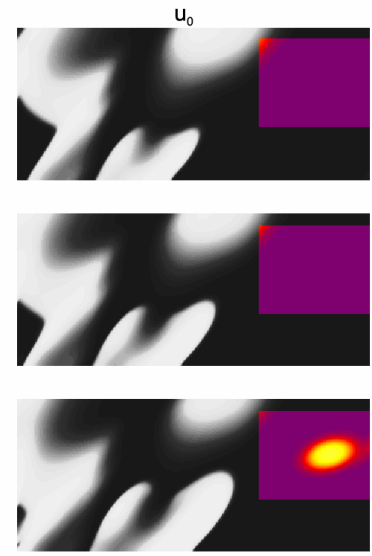
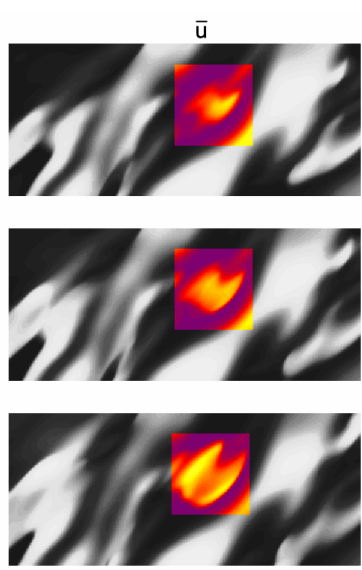

$\bar{u}$
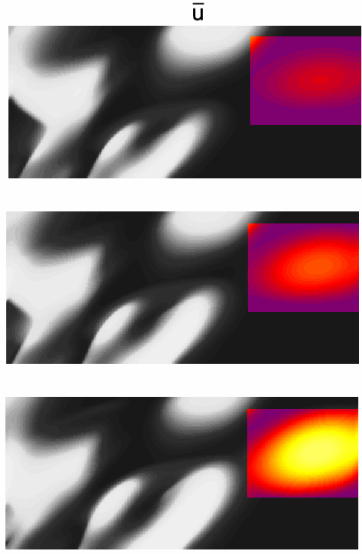

B
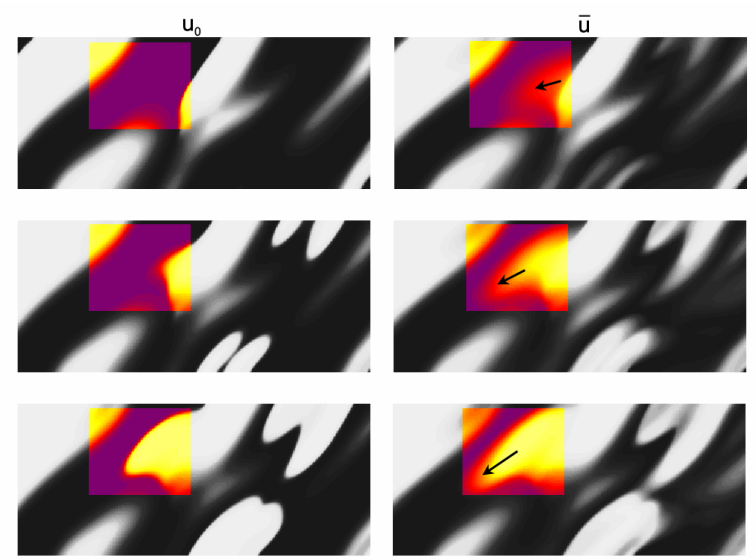

D
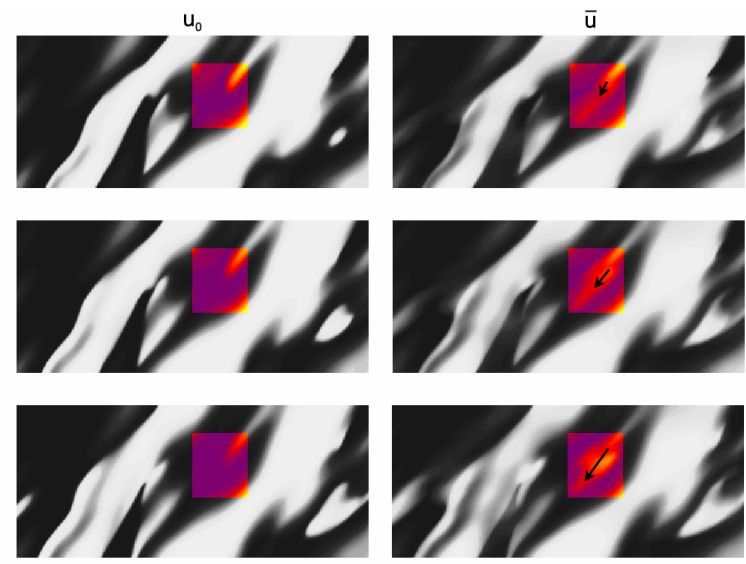

Figure 7.5: Comparison of $\bar{u}$ and $u_{0}$ signals during spiral wave breakup. Regions of interest are colored to highlight details. (A) Diminished $\bar{u}$ magnitude; (B) apparent supra-maximal conduction velocity in $u_{0}$, arrow shows direction of propagation; (C) epicardial breakthrough; (D) sub-epicardial graded potentials in $\bar{u}$; arrow shows direction of sub-epicardial propagation.

$\mathrm{ms}$, however, we have returned to a scroll wave configuration much like that of $t=0$, with the same resultant effects on $\bar{u}$.

\subsubsection{3-D scroll wave breakup in presence of fiber rotation}

For our final study, we examined a rectangular slab with rotational anisotropy, using $\theta_{\Sigma}=$ $120^{\circ}$ as a typical value; as indicated by Eq.(7.7), the fibers at the mid-wall are parallel to the $x$ axis. The simulation was conducted on a grid composed of $349 \times 151 \times 61$ grid points, corresponding to a tissue slab with dimensions of $4.6 \times 2.0 \times 0.8 \mathrm{~cm}$. However, it has been shown that a perturbed scroll wave using the MLR model in tissue up to $12 \mathrm{~mm}$ thick and $24 \% \mathrm{~mm}$ of fiber rotation fails to destabilize [24]. Therefore, we chose to use the MBR parameter 
values for Eq. (7.1) for which [24] showed that a single filament does break up for the wall thickness and rotation rate values selected for our study (see their Fig. 8).

In Figure 7.5, we show the scroll wave breakup produced from a single filament in the presence of fiber rotation. Even though the parameters of the simulation are closer to that of actual myocardium, the trends observed from sections 7.4.1 and 7.4.2 are all present in this case.

(a) The $\bar{u}$ potential is significantly lower in magnitude than that of $u_{0}$. In many regions, depolarized tissue is present at, or close to, the epicardium, while the tissue underneath is in the process of repolarizing and is returning to rest. Although the transmural layers have a smaller weight associated with them, they contribute more to $\bar{u}$ than the epicardial layers in this case, resulting in a reduction in $\bar{u}$. In Fig. $7.5 \mathrm{~A}$, we observe an epicardial breakthrough activation propagating in a $\mathrm{V}$-shaped pattern. In $u_{0}$ the depolarization is uniform over the region of breakthrough. In contrast, the activation in $\bar{u}$ is clearly of lower amplitude except in those regions where the action potential is reentering into the transmural epicardium (the legs of the V-pattern), and thereby allowing more layers to contribute to the weighted signal.

(b) A significant degree of directional blurring of the $\bar{u}$ potential. In regions where a scroll wavefront approaches the epicardium at a shallow angle, the encroaching wave exhibits a graded potential over a wide area in the direction of the wavefront in $\bar{u}$, producing a subsequent rapid depolarization and an apparent supra-maximal velocity in $u_{0}$. Figure $7.5 \mathrm{~B}$ illustrates an apparent "finger" of depolarization in $\bar{u}$ directed towards the left (shown by arrows), that the epicardial wave in $u_{0}$ subsequently follows as it breaks through.

(c) Gradual depolarization of $\bar{u}$ during epicardial breakthroughs. If a filament is oriented parallel to the epicardial surface, the signature of the rising scroll wave is observable at the epicardium in $\bar{u}$ prior to $u_{0}$. An example is shown in Fig. $7.5 \mathrm{C}$, where the $u$ time trace of the point directly over the breakthrough would demonstrate the apparent initiation of depolarization much earlier in $\bar{u}$ than in $u_{0}$.

(d) Graded potential in $\bar{u}$. In some regions, the intramural scroll wave produces a graded potential as seen in $\bar{u}$. In Fig. $7.5 \mathrm{D}$, we observe a slender region of repolarization at the top right of the colored region of interest in $u_{0}$. What is seen in $\bar{u}$ but not in $u_{0}$ is a graded potential extending retrograde to the repolarization tail (shown by arrows). In this intervening region, the wave fails to achieve an epicardial breakthrough (and hence there is no noticeable action potential in $u_{0}$ ), but is sufficiently close to the surface such that it produces an apparent 
depolarizing deflection in $\bar{u}$. Once the conduction block has sufficiently dissipated, the graded potential manifests as a breakthrough (in this case, the one shown in A). This situation is akin to the double-humped potential observed in the previous section and seen in [14].

It stands to reason that the combination of the above phenomena would lead to differences in the efficacy of the detection and subsequent tracking of the intersection of the filament with the epicardial surface (i.e., a phase singularity). We investigated this effect by examining phase singularity localization for both $u_{0}$ and $\bar{u}$ for the previous fibrillatory computation. Since an experimenter usually has access to only one variable at a time when observing epicardial behavior, rather than use the multivariate state-space phase portrait $(u, v)$ as previously, we use the Takens time-embedding state-space methodology to produce a topologically equivalent time-series phase portrait [20,25]. This is done by expanding the observed variable $u$ into a vector time series by embedding a time delay $\tau$ into $u(t)$ such that the state space in two dimensions is composed of $u(t)$ plotted against $u(t-\tau)$. We set the timeembedding delay $\tau$ to a value approximately equal to the upstroke duration of the action potential, i.e., $5 \mathrm{~ms}$, to insure the absence of folding in the phase space trajectories [26]. The origin in $(u(t), u(t-\tau))$ state-space was chosen as $(0.21,0.21)$ by examining the phase portrait for a 2-D spiral wave and selecting a point which was encircled by the smaller phase trajectories. The phase singularities were localized with our topological charge algorithm detailed previously [22].

In general, we noted several trends in singularity creation and annihilation which were seen to follow primarily as a consequence of the observations detailed above:

(a) In those regions where the magnitude of $\bar{u}$ is greater than that of $u_{0}$, the creation of the singularities is generally observed to occur earlier in $\bar{u}$ than in $u_{0}$. As mentioned previously, the sub-epicardial activation causes a premature initiation of the upstroke in $\bar{u}$ as opposed to $u_{0}$, which leads to an apparent earlier phase resetting when a singularity is produced. Likewise, the annihilation of a singularity pair typically takes place later in $\bar{u}$ than in $u_{0}$ in these regions since the underlying depolarization also persists longer in $\bar{u}$ than in $u_{0}$. 

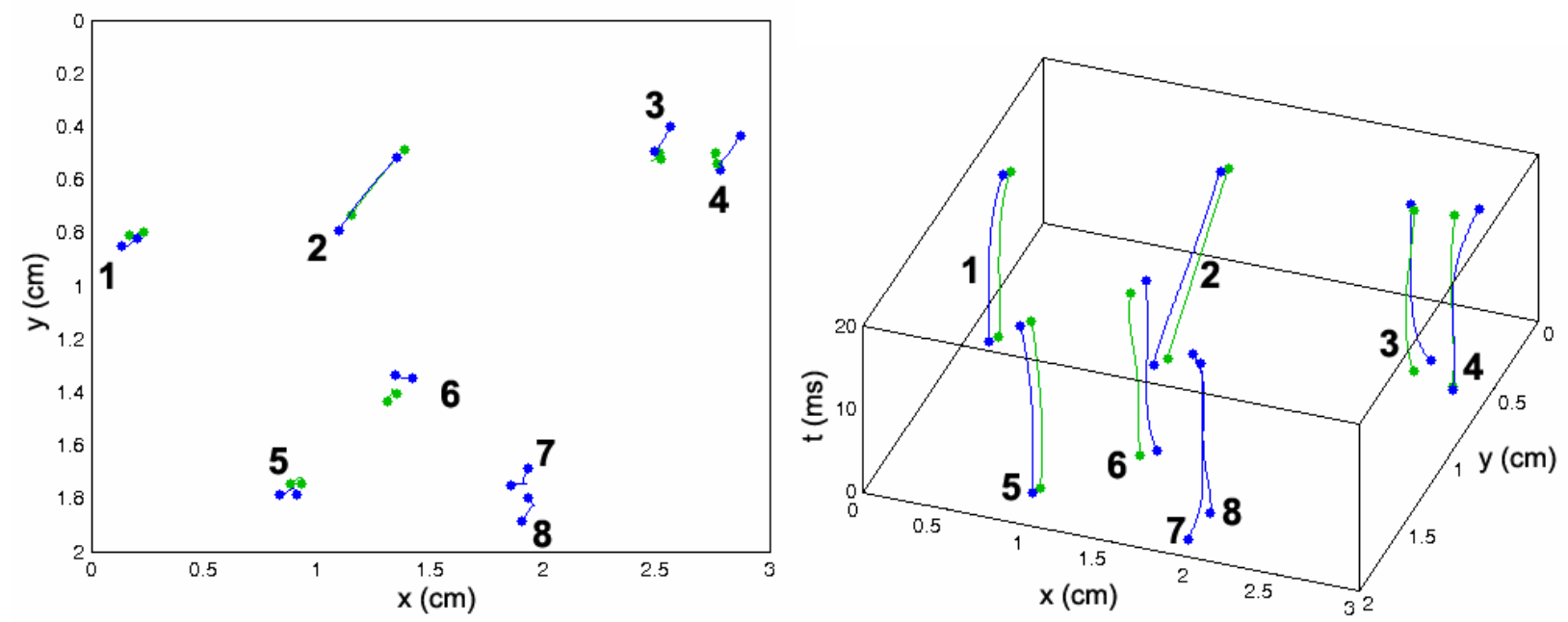

Figure 7.6: Illustration of phase singularity localization during a 20-ms segment of fibrillatory activity in the MBR model. Left panel: $(x, y)$ domain; right panel: $(x, y, t)$ domain. Trajectories from $u_{0}$ and $\bar{u}$ shown in blue and green, respectively. The singularities are labeled with numbers.

(b) In contrast, in those regions where the magnitude of $\bar{u}$ is less than that of $u_{0}$, the singularities are created generally later and annihilate earlier in $\bar{u}$ than in $u_{0}$. In this case the activated (whether depolarizing or repolarizing) epicardial tissue is overlying tissue that is close to rest, but the same reasoning in terms of apparent phase resetting applies as in (a).

(c) As an extension of (a), regions which experience graded potentials in $\bar{u}$ are likely to exhibit the appearance of singularities in $\bar{u}$ which are absent in $u_{0}$. In such areas, the apparent propagation in $\bar{u}$ is not in fact epicardial at all and hence, while the singularity may be visible in $\bar{u}$, it does not actually represent a filament intersecting with the epicardial surface but instead the trajectory of a filament has brought a section of it just underneath the surface. (d) Similarly, as an extension of (b), regions in which the activation is localized to the epicardial layer and layers immediately beneath it may reveal singularities in $u_{0}$ which are absent in $\bar{u}$. This may occur for a very shallow U-shaped filament, for example.

An example of the disparity in detection is illustrated in Fig. 7.6, which shows eight epicardial singularities in $u_{0}$ and $\bar{u}$ tracked during a $20 \mathrm{~ms}$ period. In this instance, the error in singularity localization between $u_{0}$ and $\bar{u}$ over this period is $0.57 \pm 0.16 \mathrm{~mm}$. Of note are trajectories 7 and 8 which were detected in $u_{0}$ but not in $\bar{u}$, corresponding to situation (c) above. Because the immediately underlying filament activity can produce adjacent areas which are subject to a combination of the above conditions, all of which are reflected in $\bar{u}$, it is difficult to generate 
hard-and-fast rules to determine which of the above scenarios will occur. It should be noted that in many cases the qualitative behavior of the detected phase singularities remained basically the same between $\bar{u}$ and $u_{0}$. That is, for meandering singularities which persisted for a long time period, even though discrepancies in spatial localization and creation/annihilation times existed, the basic trajectory did not change, such as trajectory 2 in Fig. 7.6.

\subsection{Discussion}

The presence of a depth-dependent optical signal has been well-documented in the literature $[27,28]$ but its effects on the interpretation of the optically mapped cardiac signal have only recently been a topic of investigation $[8,9]$. Generally speaking, investigations which have attempted quantitative measurement of the dependence of emission wavelength upon penetration depth in biological tissue have yielded varying results [29], and unfortunately the same is the case in the cardiac literature. Girouard found decay constants of 0.29 and $0.43 \mathrm{~mm}$ for the excitation and emission decay constants using di-4-ANEPPS in guinea pig left ventricle (thickness: $2.25 \pm 0.20 \mathrm{~mm}$ ), with the top $500 \mu \mathrm{m}$ contributing $95 \%$ of the signal and the largest contribution coming from the uppermost $100 \mu \mathrm{m}$; however, this value was dependent upon the optical magnification used [30]. Knisley estimated that the top $300 \mu \mathrm{m}$ contributed to the majority of the optical signal for a rabbit ventricular preparation using di-4-ANEPPS (maximum thickness measured: $600 \mu \mathrm{m}$ ) [31]. Baxter et al. found optical penetration depths of 0.80 and $1.34 \mathrm{~mm}$ for the excitation and emission decay constants of di-4-ANEPPS in transmural crosssections of sheep right ventricle (thickness: $8 \mathrm{~mm}$ ) with $82 \%$ of the signal emanating from the top millimeter [19]. A study by Al-Khadra et al. observed optically-recorded (di-4-ANEPPS) action potentials from papillary muscles which had a dual-humped morphology [32]. Shielding the papillary muscle from the underlying septum yielded action potentials of normal shape, indicating that the optical signal was the sum of the signals from the two tissues. Assuming this is the case, the underlying signal originated from a tissue depth on the order of a millimeter. Similarly, a Monte Carlo simulation by Ding et al. using broad field illumination for tissue stained with di-4-ANEPPS calculated that that $62 \%$ of the collected fluorescence originated within a 1-mm radius block of tissue with a 1-mm depth [33]. Quantitative measurements on the tissue depth which contributes to the signal have been shown to be dependent on such factors as spectral sensitivity, light absorption and scattering, among others [29,34]. Recent publications 
have called increasing attention to the need to be aware of the location of the focal plane with respect to the tissue surface as well as the associated depth of field used at a given magnification with the collecting lens [19,35].

In this study, we do not attempt to demonstrate the validity of the numerical estimates of the weighting functions mentioned in the literature. Instead, we report the expected effects of applying the weighting function of [19] on observed epicardial activation patterns, allowing inferences to be drawn with other, similar $w(z)$ functions. Using the function in [19], Janks and Roth [36] demonstrated that the inclusion of optical averaging with depth in a simulation of a passive membrane model may provide a partial explanation as to why experiments fail to observe the large transmembrane polarization near the electrode during unipolar stimulation (as predicted theoretically), and surmised that a longer length constant, $\lambda$, in $w(z)$ would increase the discrepancy. Likewise, observing active propagation with a larger value of $\lambda$ would most likely alter the results in sections 7.4.1 and 7.4.2 in a quantitative (not qualitative) manner. However, it is doubtful that the effect of a longer $\lambda$ on fibrillatory tissue, such as that seen in the last section of the Results section, would be as predictable as in [36]. As noted in [19], a sub-epicardial wave becomes noticeable when the wave approaches $1-2 \mathrm{~mm}$ of the surface using their formulation of $w(z)$. For this reason, an intramural filament produced dual-peaked APs in $\bar{u}$ only when the line of conduction block brought the trajectory of the filament very close to the epicardium. Obviously, in regards to our observations of a single shallow filament, a longer $\lambda$ would permit deeper filaments to be observed epicardially, accompanied by even longer upstroke times and larger magnitude second peaks in the filament axis. However, in the case of full fibrillatory activity, the effect of a longer $\lambda$ is more difficult to estimate due to the decreased relative difference between $\lambda$ and the scroll wavelength in the $z$ direction. That is, if more waves are present in those layers which contribute significantly to $\bar{u}$ (as determined by $\lambda$ ), the activation seen at the surface will seem to become increasingly complex as more of the total filament length (along with the associated twist due to fiber rotation and resultant scroll activity [24]) is represented in $w(z)$.

The presence of a depth-dependent signal adds a new layer of difficulty to the already complex interpretation of cardiac activation patterns. It is well known that since the optical signal is generated from an aggregate of cells, the action potential upstroke can be blurred within 
a set of pixels and erroneously lead to an artificially long upstroke duration; for CCD cameras, blurring also increases as a function of the photon integration time required to obtain an adequate SNR [30,37]. These results indicate that even if these technical effects were corrected, blurring would still occur and may be significant depending on the underlying filament orientation.

Baxter et al. notes that their equation for $w(z)$ takes into account scattering losses at the surface but not the effects of lateral scattering within the tissue; the effect of the inclusion of this behavior on our results is unknown, but will most likely further complicate optical data analysis.

The appearance of action potentials with two distinct peaks ("double-humps") has long been known as a signature of reentrant conduction block [38,39] and has been used previously for core identification [40]. Typically, these double-humped potentials are attributed to electrotonic interaction across the line of block. However, Efmov et al. demonstrated double potentials at distances greater than a space constant (up to $12 \mathrm{~mm}$ ) from the line of block [14]. Since this is a longer distance that can be normally accounted for by electronus, this led them to consider the possibility that a scroll wave may be rotating sufficiently close to the epicardial surface in such a way that the optical signal experiences two deflections from the wave propagating on either side of the region of conduction block. Our simulations confirm this interpretation as a possibility since a filament is unlikely to elicit a noticeable isolated passive response in $u_{0}$ unless it passes within a fraction of a millimeter of the epicardium (in which case a minor perturbation in the filament trajectory towards the bounding surface will produce a full active response on the epicardium), whereas the deflection in $\bar{u}$ can be observed at a tissue depth of an order of magnitude larger the passive length constant. However, while we were able to replicate the double-humped behavior in $\bar{u}$ in the controlled case of a single elliptical filament, such multiple peaks were observed to be fairly infrequent in the simulation of filament breakup. This is understandable given that the recordings made by Efimov et al. were of sustained polymorphic tachycardia as opposed to full fibrillatory activity, indicating that just a single filament was present [11]; the dynamic activity of multiple filaments precludes the stability needed to produce the extra peak in our simulations. Also, it is important to note that in the experimental setting, other factors come into play such as motion artifacts and early afterdepolarizations associated with some pathological states which may lead to the erroneous presence of multiple peaks in the optical signal. 
The detection and tracking of surface phase singularities remains the most straightforward means to discern filament activity, albeit in a limited way [11,17]. While various methodologies exist to localize singularities (see [22] for a short review), all of them assume that the epicardial mapping is in fact an accurate representation of the surface activation dynamics. We have shown here that the inclusion of optical signal depth information can lead to an erroneous characterization of the phase singularity (and hence filament) dynamics, including the occurrence of false positive and false negative filament detection under certain conditions. We note again that the basic meander pattern and trajectory is often unaffected, and if the scroll wave is fairly perpendicular to the epicardium (i.e., transmurally) within the length constant $\lambda$, then the difference in localization (though probably not the creation/annihilation times) between $\bar{u}$ and $u_{0}$. will be small. However, the presence of twist due to rotational anisotropy or other heterogeneities will promote filament bending, thus making such an optimal configuration transient at best, especially once breakup events begin to occur.

In summary, our results indicate that the depth-dependent optical signal has significant consequences on the interpretation of propagation dynamics, in terms of the distortion of the magnitude, spatial extent and temporal timing of epicardial activation and, as a consequence, phase singularity localization and behavior. As mentioned above, many additional variables clearly play a role in determination of the weighting function but their quantitative values remain unknown or inconsistent thus far; furthermore, it is difficult to reconcile the variability between experiments performed with different protocols. Nevertheless, this study illustrates that accounting for the three-dimensional nature of the optical signal is vital to appropriately bridging the gap between numerical simulations and experimental optical data in understanding cardiac spatiotemporal electrodynamics.

\subsection{Acknowledgments}

We would like to thank Michael Miga for his input into the computational methodology of this manuscript. This work was supported in part by a graduate research fellowship from the UNCF-Merck Science Initiative and a NIH National Research Service Award. 


\section{7. $\quad$ References}

[1] Sharma, V. and Tung, L., "Theoretical and experimental study of sawtooth effect in isolated cardiac cell-pairs." J Cardiovasc Electrophysiol, vol. 12, no. 10, pp. 1164-1173, 2001.

[2] Knisley, S. B., Blitchington, T. F., Hill, B. C., Grant, A. O., Smith, W. M., Pilkington, T. C., and Ideker, R. E., "Optical measurements of transmembrane potential changes during electric field stimulation of ventricular cells." Circ Res, vol. 72, no. 2, pp. 255-270, 1993.

[3] Choi, B.-R. and Salama, G., "Simultaneous maps of optical action potentials and calcium transients in guinea-pig hearts: mechanisms underlying concordant alternans." J Physiol (Lond), vol. 529, no. 1, pp. 171-188, 2000.

[4] Bray, M.-A., Lin, S.-F., and Wikswo, J. P., Jr., "Three-dimensional surface reconstruction and fluorescent visualization of cardiac activation." IEEE Trans Biomed Eng, vol. 47, no. 10, pp. 1382-1391, 2000.

[5] Salzberg, B. M., Obaid, A. L., and Benzanilla, F., "Microsecond response of a voltagesensitive merocyanine dye: fast voltage-clamp measurements on squid giant axon." Jpn $J$ Physiol, vol. 43 Suppl 1 pp. S37-S41, 1993.

[6] Grinvald, A., Lieke, E., Frostig, R. D., Gilbert, C. D., and Wiesel, T. N., "Functional architecture of cortex revealed by optical imaging of intrinsic signals." Nature, vol. 324, no. 6095, pp. 361-364, 1986.

[7] Entcheva, E., Lu, S. N., Troppman, R. H., Sharma, V., and Tung, L., "Contact fluorescence imaging of reentry in monolayers of cultured neonatal rat ventricular myocytes." J Cardiovasc Electrophysiol, vol. 11, no. 6, pp. 665-676, 2000.

[8] Roth, B. J., "Artifacts, assumptions, and ambiguity: Pitfalls in comparing experimental results to numerical simulations when studying electrical stimulation of the heart." Chaos, vol. 12, no. 3, pp. 973-981, 2002.

[9] Gray, R. A., "What exactly are optically recorded "action potentials"?" J Cardiovasc Electrophysiol, vol. 10, no. 11, pp. 1463-1466, 1999.

[10] Winfree, A. T., When Time Breaks Down: The Three-Dimensional Dynamics Of Electrochemical Waves And Cardiac Arrhythmias. Princeton: Princeton University Press, 1987.

[11] Gray, R. A., Jalife, J., Panfilov, A., Baxter, W. T., Cabo, C., Davidenko, J. M., and Pertsov, A. M., "Nonstationary vortexlike reentrant activity as a mechanism of polymorphic ventricular tachycardia in the isolated rabbit heart." Circulation, vol. 91, no. 9, pp. 2454-2469, 1995. 
[12] Winfree, A. T., Caudle, S., Chen, G., McGuire, P., and Szilagyi, Z., "Quantitative optical tomography of chemical waves and their organizing centers." Chaos, vol. 6, no. 4, pp. 617-626, 1996.

[13] Pertsov, A., Vinson, M., and Müller, S. C., "Three-dimensional reconstruction of organizing centers in excitable chemical media." Physica D, vol. 63, no. 1-2, pp. 233240, 1993.

[14] Efimov, I. R., Sidorov, V., Cheng, Y., and Wollenzier, B., "Evidence of threedimensional scroll waves with ribbon-shaped filament as a mechanism of ventricular tachycardia in the isolated rabbit heart." J Cardiovasc Electrophysiol, vol. 10, no. 11, pp. 1452-1462, 1999.

[15] Efimov, I. R., Aguel, F., Cheng, Y., Wollenzier, B., and Trayanova, N., "Virtual electrode polarization in the far field: implications for external defibrillation." Am J Physiol Heart Circ Physiol, vol. 279, no. 3, pp. H1055-H1070, 2000.

[16] Choi, B.-R. and Salama, G., "Optical mapping of atrioventricular node reveals a conduction barrier between atrial and nodal cells." Am J Physiol, vol. 274, no. 3 Pt 2, pp. H829-H845, 1998.

[17] Fenton, F. and Karma, A., "Vortex dynamics in three-dimensional continuous myocardium with fiber rotation: Filament instability and fibrillation." Chaos, vol. 8, no. 1, pp. 20-47, 1998.

[18] Roth, B. J., "Electrical conductivity values used with the bidomain model of cardiac tissue." IEEE Trans Biomed Eng, vol. 44, no. 4, pp. 326-328, 1997.

[19] Baxter, W. T., Mironov, S. F., Zaitsev, A. V., Jalife, J., and Pertsov, A. M., "Visualizing excitation waves inside cardiac muscle using transillumination." Biophys $J$, vol. 80, no. 1, pp. 516-530, 2001.

[20] Gray, R. A., Pertsov, A. M., and Jalife, J., "Spatial and temporal organization during cardiac fibrillation." Nature, vol. 392, no. 6671, pp. 75-78, 1998.

[21] Bray, M.-A., Lin, S.-F., Aliev, R. R., Roth, B. J., and Wikswo, J. P., Jr., "Experimental and theoretical analysis of phase singularity dynamics in cardiac tissue." $J$ Cardiovasc Electrophysiol, vol. 12, no. 6, pp. 716-722, 2001.

[22] Bray, M.-A. and Wikswo, J. P., "Use of topological charge to determine filament location and dynamics in a numerical model of scroll wave activity." IEEE Trans Biomed Eng, vol. 49, no. 10, pp. 1086-1093, 2002.

[23] Pertsov, A. M., Davidenko, J. M., Salomonsz, R., Baxter, W. T., and Jalife, J., "Spiral waves of excitation underlie reentrant activity in isolated cardiac muscle." Circ Res, vol. 72, no. 3, pp. 631-650, 1993. 
[24] Rappel, W.-J., "Filament instability and rotational tissue anisotropy: A numerical study using detailed cardiac models." Chaos, vol. 11, no. 1, pp. 71-80, 2001.

[25] Takens, F., "Detecting strange attractors in turbulence.," in Rand, D. A. and Young, L.-S. (eds.) Dynamical Systems and Turbulence Berlin: Springer-Verlag, 1981, pp. 366-381.

[26] Gray, R. A. and Jalife, J., "Video imaging of atrial defibrillation.," in Zipes, D. P. and Jalife, J. (eds.) Cardiac Electrophysiology: From Cell to Bedside 3rd ed. Philadelphia: W.B. Saunders, 1999, pp. 432-439.

[27] Wilson, B. C., Jeeves, W. P., and Lowe, D. M., "In vivo and post mortem measurements of the attenuation spectra of light in mammalian tissues." Photochem Photobiol, vol. 42, no. 2, pp. 153-162, 1985 .

[28] Preuss, L. E., Bolin, F. P., and Cain, B. W., "A comment on spectral transmittance in mammalian skeletal muscle." Photochem Photobiol, vol. 37, no. 1, pp. 113-116, 1983.

[29] Cheong, W. F., Prahl, S. A., and Welch, A. J., "A review of the optical properties of biological tissues." IEEE Journal of Quantum Electronics, vol. 26, no. 12, pp. 2166$2185,1990$.

[30] Girouard, S. D., Laurita, K. R., and Rosenbaum, D. S., "Unique properties of cardiac action potentials recorded with voltage-sensitive dyes." J Cardiovasc Electrophysiol, vol. 7, no. 11, pp. 1024-1038, 1996.

[31] Knisley, S. B., "Transmembrane voltage changes during unipolar stimulation of rabbit ventricle." Circ Res, vol. 77, no. 6, pp. 1229-1239, 1995.

[32] Al-Khadra, A., Nikolski, V., and Efimov, I. R., "The role of electroporation in defibrillation." Circ Res, vol. 87, no. 9, pp. 797-804, 2000.

[33] Ding, L., Splinter, R., and Knisley, S. B., "Quantifying spatial localization of optical mapping using Monte Carlo simulations." IEEE Trans Biomed Eng, vol. 48, no. 10, pp. 1098-1107, 2001.

[34] Baxter, W. T., "Optical Properties of Cardiac Tissue.," in Rosenbaum, D. S. and Jalife, J. (eds.) Optical Mapping of Cardiac Excitation and Arrhythmias Armonk: Furtura Publishing Company, 2001, pp. 47-59.

[35] Efimov, I. R., "What is the role of the AV node if the AV delay occurs before it?" Am J Physiol, vol. 275, no. 5 Pt 2, pp. H1905-H1906, 1998.

[36] Janks, D. L. and Roth, B. J., "Averaging over depth during optical mapping of unipolar stimulation." IEEE Trans Biomed Eng, vol. 49, no. 9, pp. 1051-1054, 2002.

[37] Baxter, W. T., Davidenko, J. M., Loew, L. M., Wuskell, J. P., and Jalife, J., "Technical features of a CCD video camera system to record cardiac fluorescent data." Ann Biomed Eng, vol. 25, no. 4, pp. 713-725, 1997. 
[38] Allessie, M. A., Bonke, F. I. M., and Schopman, F. J. G., "Circus movement in rabbit atrial muscle as a mechanism of tachycardia: III. The "leading circle" concept. A new mode of circus movement in cardiac tissue witout the involvement of an anatomical obstacle." Circ Res, vol. 41 pp. 9-18, 1977.

[39] Olshansky, B., Moreira, D., and Waldo, A. L., "Characterization of double potentials during ventricular tachycardia. Studies during transient entrainment." Circulation, vol. 87, no. 2, pp. 373-381, 1993.

[40] Jalife, J., Gray, R. A., Morley, G. E., and Davidenko, J. M., "Self-organization and the dynamical nature of ventricular fibrillation." Chaos, vol. 8, no. 1, pp. 79-93, 1998. 


\section{CHAPTER VIII}

\section{SUMMARY AND FUTURE WORK}

The objectives of this research were the development and utilization of new techniques to analyze optical recordings of cardiac spiral wave and scroll wave activity, both in the context of local electrodynamic behavior and global large-scale behavior.

The review of the dynamic characteristics of cardiac tissue in Chapter 2 served to illustrate the magnitude and importance of cardiac arrhythmia research. The spiral wave was established as the basic unit of fibrillation and tachycardia. After investigating the fact that spiral wave motion and interaction dynamics is an open question in the field, the three-dimensional nature of the problem was described and the concept of the filament was presented. The use of the phase portrait as a tool to examine a non-linear dynamic system and its application to the general principles of topological charge and phase singularities were introduced. Finally, optical recording was presented as a recent innovation to experimental observation.

Within this framework, Chapter 3 detailed the development of an algorithm which detects phase singularities based upon calculation of the two-dimensional phase distribution. This procedure is based upon the link between the curl of the phase gradient and the definition of topological charge. By discretizing the topological charge path integral, the algorithm is able to isolate the phase singularities in an efficient and robust manner from both experimental and numerically simulated data. An initial comparison of experimental singularity behavior revealed that the separation distances between singularities in quatrefoil reentry could be grouped into classes but these categories were at variance with numerically predicted results.

While the topological charge algorithm is mathematically sound, it is only as effective as the phase calculation which precedes it. Chapter 4 refined the methodology involved in reconstructing the phase portrait and the definition of the point around which the phase is defined. The impetus was to attempt to overcome some of the limitations of using time-series analysis and utilize some of the characteristics of the action potential morphology in determining the phase portrait origin. The combination of the Hilbert transform and the pEMD enabled the observation of singularities closer to their initial formation during quatrefoil reentry than with the method shown in Chapter 3. 
Phase singularities are the cross-sections of three-dimensional filaments, the behavior of which is considered integral to an understanding the transition to turbulence during fibrillation. Chapter 5 generalizes the algorithm described in Chapter 3 to the three-dimensional spatial domain. In addition, the time-series analytical method of phase portrait reconstruction was compared against two-variable phase portrait in terms of localization. Finally, since sophisticated cardiac models are multivariate, the study provided the criteria for determining which two variables are used for an optimal phase portrait.

With the interaction of phase singularities and filaments unknown in all but a select few analytical models, Chapter 6 attempted to address the discrepancy in experimental and theoretical results from Chapter 3. Quantification of the interaction of a simplified filament system in a configuration similar to that generated by quatrefoil reentry in terms of filament lifetime indicated that a critical bifurcation exists between attractive and repulsive behavior along with annihilation by mutual collision and collapse by shrinkage. The possibility of treating the filaments as a pair of point charges by fitting the initial velocities to a Yukawa potential was also investigated.

Optical recording has become the de facto means of obtaining high-resolution cardiac data. For a typical epicardial recording, it has generally been believed that the thickness of tissue which contributes to the optical signal is on the order of $250 \mu \mathrm{m}$. However, this is an assumption which has increasingly been challenged. Chapter 7 is an attempt to incorporate the attenuation with depth of the optical signal into the observation of filament and singularity dynamics. This study suggests that the inclusion of optical depth leads to a variety of significant effects, such as long upstroke times, graded potentials stemming from the underlying filaments, and false positive and negative singularity detection.

Appendix A1 provided a demonstration that singularity detection on a whole-heart basis is indeed possible. The catadioptric system that was developed previously has been shown to be a viable system for the visualization of the entire epicardial surface [1] but its application to the issue of small-scale cardiac electrodynamics has not been performed until now.

\subsection{Limitations and future work}

A number of limitations within this study need to be mentioned. The topological charge algorithm described in Chapter 3 is designed for a rectangular grid; this formulation is sufficient 
for most applications which use rectilinear coordinates. However, some numerical models require the use of unstructured finite meshes, for which the line integral is not as straightforward to compute. This critique also applies to the generalized algorithm described in Chapter 5 .

Regarding Chapter 4, the heuristic used to reconstruct the pEMD remains empirical in nature; there is not yet a rigorous rule to determine the critical amplitude and peak-to-peak width for a double potential response characteristic of an excitable element in the vicinity of the core. The use of the pEMD which is dependent on the non-local characteristics of the signal also means that it is not amenable to real-time analytical use, as opposed to the time-series method which only requires retention of data frames from the previous $\tau$ time-units. For this reason, the Hilbert-pEMD algorithm might be impractical on large data sets because an additional variable the same size as the original needs to be generated. Also, it is important to consider that the pEMD is a global operator based upon the extrema of the voltage signal; while the pEMD signal and the pEMD-Hilbert transformed signal may be independent, it is not one-sided with respect to time. Therefore, trends in the pEMD that occur prior to a particular point in time may be reflected in the phase values at that time point or even afterwards, such that the phase values are causally related to preceding trends as well as current events.

Generally speaking, the choice of the origin in the phase portrait is the most critical issue in obtaining the phase of a system by this method. Several studies have used a single fixed point to calculate phase around, such the mean of each pixel [2-6]; the pEMD in Chapter 4 is a temporally-dependent determination for non-stationary spirals. It is worth noting that a previous study has shown that an amplitude range exists over which the error in singularity localization is fairly small [6]. Selecting the origin for a 1-D numerical system is simple: find the fixed point by solving for the intersection of the nullclines since all phase trajectories encircle this point. However, for a spatially-coupled case, the solution is not as straightforward since the diffusive term offsets the phase trajectories with respect to the 1-D case; therefore, the phase trajectories may not encircle a single point. Furthermore, this offset is dependent on the value of the laplacian and hence is not consistent between spatial locations, or even between successive time steps if the reentrant pattern is non-stationary. In such instances, if the pEMD is not practical, the question remains of how to determine the best starting estimate for the origin; one possible criterion is choosing a value close to the excitation threshold [7]. These concerns are worth exploring in more detail. 
Adding to the difficulty of validating the methods presented here is the fact that while there is a variety of filament/singularity detection techniques (see the Introduction section of Chapter 5 for a review), each with their own shortcomings, there is no universally accepted "gold standard" methodology. A comprehensive quantitative comparison of these methods would be beneficial to the cardiac community.

The filament interaction analysis presented in Chapter 6 includes a number of simplifications. Rather than using one of the standard cardiac models, this portion of the study used the oscillatory BZ chemical reaction. The reason for this choice is that the scroll ring dynamics for a doubly-diffusive system of this type are well-documented and simple, in that they exhibit shrinkage only with no associated drift (provided the curvature is not excessive). This choice was deemed necessary in order to examine the interaction of the filaments such that any drift (i.e., attractive or repulsive behavior) could be attributed to the interaction and not to idiosyncrasies of the ring dynamics itself. Also, we have imposed severe symmetry constraints on the system (axisymmetric geometry, reflecting boundary conditions), again for the sake of simplicity. The behaviors of adjacent filaments during fibrillation certainly do not obey such constraints except in transient, isolated cases. Lastly, structural heterogeneities such as tissue anisotropy and fiber rotation and curvature have been ignored, all of which are known to contribute to the motion of epicardial phase singularities and filaments [8]. However, this study is a first step in this research direction and these restrictions will be relaxed in future studies.

The study of effects of optical depth on observed filament dynamics in Chapter 7 was limited primarily by the fact that the weighting function used was a model of fluence rate from flourophore point sources in terms of absorption only but not in terms of scattering. It is wellknown that scattering of light in biological tissue is appreciable, especially laterally in the myocardium; a study quantifying the amount of lateral scattering has not yet been performed. Other effects such as the location of the focal plane and the depth of field are only recently being investigated in the context of cardiac optical studies, and hence are not included in the model either.

Currently, the reconstruction of the heart geometry in Appendix A1 is limited primarily by the total time required by the process. Currently, a panoramic experiment progresses through the following steps: (1) the heart is affixed to the perfusion system, and the mirrors and camera adjusted; (2) the whole heart experiment is performed; (3) the heart is rotated on the perfusion 
system to obtain snapshots for the geometric reconstruction; care must be taken to insure that neither the camera nor the mirrors are moved; (4) the heart is removed, and a target object is put in its place to determine the mirror position with respect to the camera; (5) the mirrors are removed and a calibration grid is used to calibrate the camera. Care must be taken to insure that the camera is not jostled during steps $1-5$ as well as the mirrors during steps $1-4$. Generally, the calibration of the camera is a rate-limiting factor during the time course of the experiment. The fact that the camera calibration and determination of the mirror orientation takes place after completion of the experiment precludes creating the geometric model for real-time use during the experiment. An ideal system would permit quick and precise calibration of the camera and mirrors at the beginning of the experiment. Many of the current difficulties with calibration stem from the fact that the imaging optics and the object to be imaging are positioned independently of one another. This difficulty can be overcome if all components of the imaging system could be placed on a stable mounting system; in this way, calibration needs to be performed only once. In addition, currently each incremental rotation of the perfusion system and each snapshot for the reconstruction must be individually entered at a console; an automatic computerized means of staggering the rotation and the snapshot acquisition would streamline the system and reduce total run time of the experiment.

\subsection{Research considerations}

Before beginning this research, two major factors were considered: how to protect the research subjects used in this research, and what, if any, contributions this research would make to society. These factors are described below:

1) Protection of research subjects: No human subjects were used for this research. As much of the research was theoretical in nature, no animals were used directly since much of the data was generated by numerical modeling. Numerical modeling has been integral to the discipline of cardiac electrophysiology, both as an impetus to suggest new experiments and as a means to provide insight into the physics of cardiac action potential propagation, in particular, the phenomenon of reentry. Most of the experimental results presented were derived or validated using rabbit cardiac data obtained from earlier experiments [9]. For these cases, experiments were conducted in accordance with NIH regulations for the ethical use of animals in research and were approved by the Vanderbilt University Institutional Animal Care and Use Committee 
(IACUC approval date: 8/14/01, Animal Welfare Assurance Number: A3227-0). As new and interesting quantities arise, new techniques will be implemented to examine them and the present method of re-examining previous data to validate these techniques has served well and will most likely continue to do so.

2) Societal implications: The phenomenon of cardiac fibrillation continues to be the primary cause of mortality in the industrialized world [10]. Advances in treatment and prevention are largely guided by gaining a better understanding of the initiation, subsequent behavior and interaction, and annihilation of reentrant waves and the phase singularities which they organize around. In terms of applications to engineering, the algorithm to detect topological charge distribution has the advantage of mathematical robustness and computational speed. In terms of novel scientific research, the interaction of filaments is still a largely unexplored field and our studies have added to the gradually growing body of literature. In addition, the issue of optical depth with respect to optical mapping is still in its infancy and the results presented here indicate that it should be a topic of interest to cardiac electrophysiologists.

\subsection{References}

[1] Bray, M.-A., Lin, S.-F., and Wikswo, J. P., Jr., "Three-dimensional surface reconstruction and fluorescent visualization of cardiac activation." IEEE Trans Biomed Eng, vol. 47, no. 10, pp. 1382-1391, 2000.

[2] Gray, R. A., Pertsov, A. M., and Jalife, J., "Spatial and temporal organization during cardiac fibrillation." Nature, vol. 392, no. 6671, pp. 75-78, 1998.

[3] Chen, J., Mandapati, R., Berenfeld, O., Skanes, A. C., and Jalife, J., "High-frequency periodic sources underlie ventricular fibrillation in the isolated rabbit heart." Circ Res, vol. 86, no. 1, pp. 86-93, 2000.

[4] Chen, J., Mandapati, R., Berenfeld, O., Skanes, A. C., Gray, R. A., and Jalife, J., "Dynamics of wavelets and their role in atrial fibrillation in the isolated sheep heart." Cardiovasc Res, vol. 48, no. 2, pp. 220-232, 2000.

[5] Samie, F. H., Berenfeld, O., Anumonwo, J., Mironov, S. F., Udassi, S., Beaumont, J., Taffet, S., Pertsov, A. M., and Jalife, J., "Rectification of the background potassium current: A determinant of rotor dynamics in ventricular fibrillation." Circ Res, vol. 89, no. 12 , pp. 1216-1223, 2001.

[6] Iyer, A. N. and Gray, R. A., "An experimentalist's approach to accurate localization of phase singularities during reentry." Ann Biomed Eng, vol. 29, no. 1, pp. 47-59, 2001. 
[7] Eason, J. and Trayanova, N., "Phase singularities and termination of spiral wave reentry." $J$ Cardiovasc Electrophysiol, vol. 13, no. 7, pp. 672-679, 2002.

[8] Fenton, F. and Karma, A., "Vortex dynamics in three-dimensional continuous myocardium with fiber rotation: Filament instability and fibrillation." Chaos, vol. 8, no. 1, pp. 20-47, 1998.

[9] Lin, S.-F., Roth, B. J., and Wikswo, J. P., Jr., "Quatrefoil reentry in myocardium: An optical imaging study of the induction mechanism." J Cardiovasc Electrophysiol, vol. 10, no. 4, pp. 574-586, 1999.

[10] Myerburg, R. J., Kessler, K. M., and Castellanos, A., "Sudden cardiac death: Structure, function and time-dependence of risk." Circulation, vol. 85, no. 1 Suppl, pp. I2-I10, 1992. 


\title{
APPENDIX A
}

\section{Three-Dimensional Visualization Of Phase Singularities On The ISOLATED RABBIT HEART}

\author{
Mark-Anthony Bray ${ }^{1}$, Shien-Fong Lin $^{2}$, and John P. Wikswo ${ }^{2}$
}

\author{
${ }^{1}$ Department of Biomedical Engineering \\ ${ }^{2}$ Department of Physics and Astronomy \\ Vanderbilt University \\ Nashville, TN 37235
}

Portions of this manuscript have been published in:

Bray, M-A, S-F Lin and JP Wikswo, Journal of Cardiovascular Electrophysiology: EP Images

Vol. 13, No. 12, pp. 1311, December 2002.

(C) 2001 by Futura Publishing Company, Inc. 


\section{A.1 Introduction: Whole-heart optical mapping}

As noted in Chapter 1, optical mapping allows for simultaneous recording of hundreds of sites. However, studies of large-scale wavefront dynamics require visualization of the entire epicardial surface. Current use of optical imaging technology is still limited to a single field of view of the heart, namely that portion of the heart that is visible to the imaging device at a given point in time. Two solutions have been proposed to this problem: the use of a system of multiple cameras to record from different regions simultaneously [1-4], or a system of reflecting surfaces (a catadioptric system). Both methods serve to maximize photodetector coverage of the full epicardial surface. The use of a catadioptric system has the following advantages over the use of multiple cameras for cardiac imaging [5]:

1. Certain mirrors, when imaged by a perspective camera, yield a single viewpoint projection of a particular scene.

2. A wide field of view can be obtained using non-planar mirrors while maintaining a single viewpoint.

3. Parameters of the lens, CCD, and digitizer, such as blurring, lens distortion, pixel size, aspect ratio, etc., are identical for all the views. For all practical purposes, each view is produced by the same camera-digitizer system. In particular, in a multi-camera setup, the cameras must be synchronized in order to present a temporally synchronous display of cardiac propagation. The use of mirrors permits capture synchronization for all views, and hence, allows for the observation of asynchronous events, such as arrhythmias and fibrillation, over the whole heart.

4. Since the setup is monocular, only one set of intrinsic camera parameters needs to be determined for camera calibration purposes.

The cost of the system setup is directly proportional to the number of cameras and digitizers used. A system with multiple cameras may be prohibitively expensive, while a monocular setup has an overhead of only one camera and one digitizer.

A special concern of optical mapping, and particularly of whole heart imaging, are artifacts due to muscular contraction; motion of the cardiac tissue may cause blurring which confounds localization of wavefront activity. Contractile motion may be halted though either mechanical or chemical means. Mechanically suspending contraction usually involves pressing the portion of the heart to be imaged against an optical window [6,7]. The primary advantage is 
the absence of pharmacological agents that could potentially alter the electrophysiological properties of the cardiac tissue that are to be measured. However, the compression of the cardiac vasculature upon the viewing window increases the possibility of ischemia of the region that is being imaged and the use of a mechanical constraining device would block some portion of the heart from the imager. The effects of tissue compression and the tissue-glass interface upon the spatial patterns of polarization are still in question [8]. Chemical agents are used more frequently in optical experiments, the primary agents being diacetyl monoxime (DAM, 2,3-butanedione monoxime), which inhibits cross-bridge interaction, and D600 (gallopamil), which blocks $\mathrm{Ca}^{2+}$ flux through the slow channel. Both have been determined to have slight nonspecific influences on action potential properties [9,10], although DAM has been reported to cause ventricular edema [11]. Recent studies indicate that cytochalasin D acts as an effective mechanical decoupler, while reducing the action potential-altering effects of DAM [12].

While a catadioptric system has been developed for whole-heart mapping [13], it was limited by producing three discontinuous images with only the anatomic features to resolve them. A multi-view geometric reconstruction algorithm has been developed to overcome these issues and was previously validated as a proof-of-concept [14]. This study represents the first attempt at three-dimensional, whole-heart phase singularity localization.

\section{A.2 Methods and Results}

We observed the three-dimensional electrodynamic behavior of a Langendorff-perfused rabbit heart stained with the voltage-sensitive dye di-4-ANEPPS during endocardial pacing and fibrillation, using panoramic epicardial imaging to provide catadioptric visualization of the anterior and posterior regions of the heart with one CCD camera and two mirrors at a frame rate of 335 frames/sec in a $128 \times 64$ pixel format [14]. The $\mathrm{Ca}^{2+}$ channel blocker D600 eliminated motion artifacts. We then created phase maps [15] from the normalized fluorescence $(F)$ using an algorithm based on the Hilbert transform [16], and generated singularity maps by computing topological charge [17]. In contrast to previous studies, with a limited field of view, we herein report panoramic visualization of not only $V_{m}$ but also cardiac phase and phase singularity distributions over the entire ventricular epicardium.

Whole-heart geometric reconstructions are shown in Fig. A.1 in panels A through C for both pacing and fibrillatory activity, with the atrial pixels removed (white circle indicates 
endocardial pacing site). The ventricular $d F / d t$ mapping is illustrated in $\mathrm{A}$, showing the wave fronts. Panel B depicts the phase mapping for the behavior in A, while panel C shows the phase singularity distribution $n_{t}$ computed from B. Note the expected absence of singularities during pacing and their presence during fibrillation. This procedure illustrates that fibrillatory wholeheart electrodynamic behavior can be described in terms of phase singularities by observing the singularities' location and dynamics relative to ventricular epicardial anatomy.

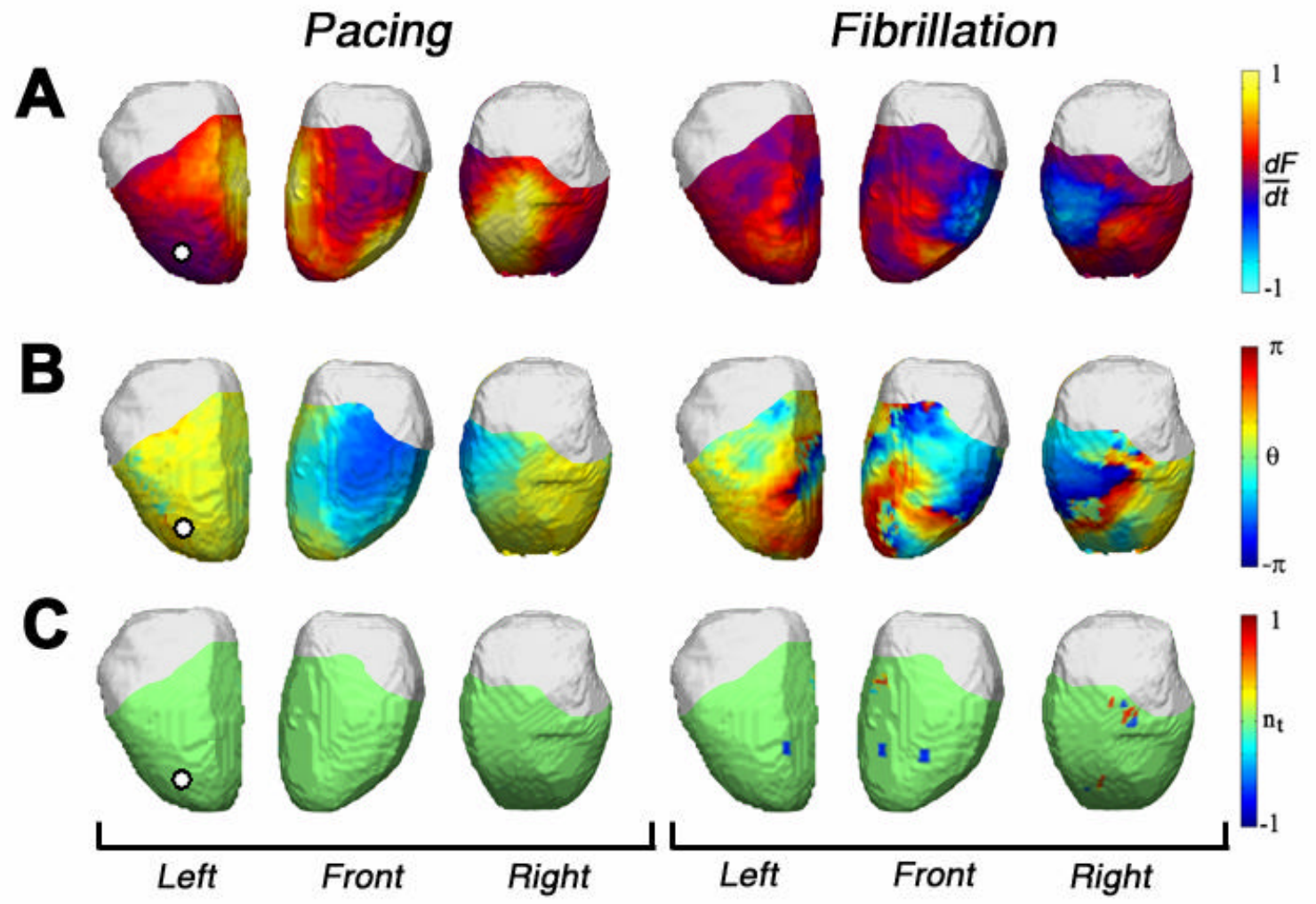

Figure A.1: Whole-heart geometric reconstructions mapped with (A) temporal derivative of fluorescence $d F / d t$, (B) phase $\theta$ and (C) singularity distribution, $n_{t}$. Pacing and fibrillation episodes shown in separate columns.

\section{A.3 Acknowledgments}

This study was supported by NIH Grant R01-HL58241 and an NSF and UNCF-Merck Graduate Research Fellowship. 


\section{A.4 References}

[1] Banville, I., Gray, R. A., and Ideker, R. E., "Shock-induced figure-eight reentry in the whole heart: An optical mapping study." Circ Res, vol. 85, no. 8, pp. 742-752, 1999.

[2] Sung, D., Omens, J. H., and McCulloch, A. D., "Model-based analysis of optically mapped epicardial activation patterns and conduction velocity." Ann Biomed Eng, vol. 28, no. 9, pp. 1085-1092, 2000.

[3] Chattipakorn, N., Banville, I., Gray, R. A., and Ideker, R. E., "Mechanism of ventricular defibrillation for near-defibrillation threshold shocks: A whole-heart optical mapping study in swine." Circulation, vol. 104, no. 11, pp. 1313-1319, 2001.

[4] Samie, F. H., Berenfeld, O., Anumonwo, J., Mironov, S. F., Udassi, S., Beaumont, J., Taffet, S., Pertsov, A. M., and Jalife, J., "Rectification of the background potassium current: A determinant of rotor dynamics in ventricular fibrillation." Circ Res, vol. 89, no. 12, pp. 1216-1223, 2001.

[5] Nene, S. A. and Nayar, S. K. Stereo with mirrors. 1087-94. 1998. Proceedings of IEEE International Conference on Computer Vision (ICCV98).

[6] Girouard, S. D., Laurita, K. R., and Rosenbaum, D. S., "Unique properties of cardiac action potentials recorded with voltage-sensitive dyes." J Cardiovasc Electrophysiol, vol. 7, no. 11, pp. 1024-1038, 1996.

[7] Witkowski, F. X., Leon, L. J., Penkoske, P. A., Giles, W. R., Spano, M. L., and Winfree, A. T., "Spatiotemporal evolution of ventricular fibrillation." Nature, vol. 392, no. 6671, pp. 78-82, 1998.

[8] Entcheva, E., Efimov, I. R., Cheng, Y., Malkin, R., and Claydon, F., "Virtual electrode effects in transvenous defibrillation-modulation by structure and interface: Evidence from bidomain simulations and optical mapping." J Cardiovasc Electrophysiol, vol. 9, no. 9, pp. 949-961, 1998.

[9] Liu, Y., Cabo, C., Salomonsz, R., Delmar, M., Davidenko, J., and Jalife, J., "Effects of diacetyl monoxime on the electrical properties of sheep and guinea pig ventricular muscle." Cardiovasc Res, vol. 27, no. 11, pp. 1991-1997, 1993.

[10] Noguchi, K., Masumiya, H., Takahashi, K., Kameko, K., Higuchi, S., Tanaka, H., and Shigenobu, K., "Comparative effects of gallopamil and verapamil on the mechanical and electrophysiological parameters of isolated guinea-pig myocardium." Can J Physiol Pharmacol, vol. 75, no. 12, pp. 1316-1321, 1997.

[11] Gray, R. A., Ayers, G., and Jalife, J., "Video imaging of atrial defibrillation in the sheep heart." Circulation, vol. 95, no. 4, pp. 1038-1047, Feb.1997.

[12] Biermann, M., Rubart, M., Moreno, A., Wu, J., Josiah-Durant, A., and Zipes, D. P., "Differential effects of cytochalasin $\mathrm{D}$ and 2,3 butanedione monoxime on isometric 
twitch force and transmembrane action potential in isolated ventricular muscle: Implications for optical measurements of cardiac repolarization." J Cardiovasc Electrophysiol, vol. 9, no. 12, pp. 1348-1357, 1998.

[13] Lin, S.-F. and Wikswo, J. P., Jr., "Panoramic optical imaging of electrical propagation in isolated heart." J Biomed Opt, vol. 4, no. 2, pp. 200-207, 1999.

[14] Bray, M.-A., Lin, S.-F., and Wikswo, J. P., Jr., "Three-dimensional surface reconstruction and fluorescent visualization of cardiac activation." IEEE Trans Biomed Eng, vol. 47, no. 10, pp. 1382-1391, 2000.

[15] Gray, R. A., Pertsov, A. M., and Jalife, J., "Spatial and temporal organization during cardiac fibrillation." Nature, vol. 392, no. 6671, pp. 75-78, 1998.

[16] Bray, M.-A. and Wikswo, J. P., "Considerations in phase plane analysis for nonstationary reentrant cardiac behavior." Phys Rev E, vol. 65, no. 5, pp. 051902, 2002.

[17] Bray, M.-A., Lin, S.-F., Aliev, R. R., Roth, B. J., and Wikswo, J. P., Jr., "Experimental and theoretical analysis of phase singularity dynamics in cardiac tissue." J Cardiovasc Electrophysiol, vol. 12, no. 6, pp. 716-722, 2001. 